\title{
Filicinic Acid Based Meroterpenoids with Anti-Epstein-Barr virus Activities from Hypericum japonicum
}

Linzhen Hu, ${ }^{\dagger,+, \|}$ Yu Zhang, ${ }^{\ddagger, \|}$ Hucheng Zhu, ${ }^{\dagger}$ Junjun Liu, ${ }^{\dagger}$ Hua Li, ${ }^{\dagger}$ Xiao-Nian Li, ${ }^{\S}$ Weiguang Sun, ${ }^{\dagger}$ Junfen Zeng, ${ }^{\perp}$ Yongbo Xue,${ }^{\dagger}$ and Yonghui Zhang ${ }^{*}, \dagger$

School of Pharmacy, Tongji Medical College, Huazhong University of Science and Technology, Wuhan 430030, P. R. China, Union Hospital, Tongji Medical College, Huazhong University of Science and Technology, Wuhan 430022, P. R. China, Kunming Institute of Botany, Chinese Academy of Sciences, Kunming 650204, P. R. China, and Department of Pharmacy, Renmin Hospital of Wuhan University, Wuhan 430060, P. R. China

\footnotetext{
Corresponding Author

*zhangyh@mails.tjmu.edu.cn (Y. Zhang)

\|These authors contributed equally to this work.
} 


\section{CONTENTS}

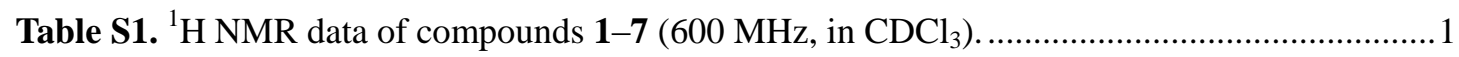

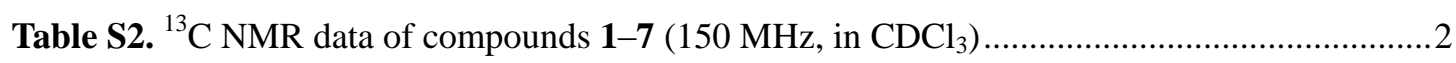

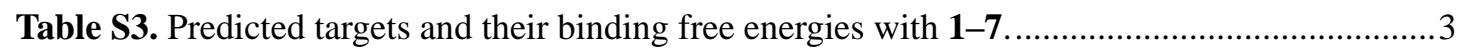

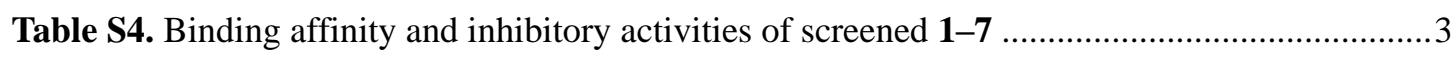

Figure S1. Three types (I-III) of phloroglucinol-terpene adducts (First row) and the skeletons of phloroglucinol-sesquiterpene adducts previous reported...........................................................

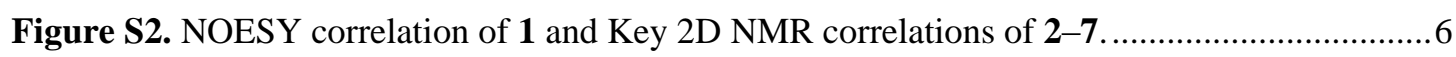

Figure S3. $\mathrm{Rh}_{2}\left(\mathrm{OCOCF}_{3}\right)_{4}$ induced $\mathrm{ECD}$ spectrum of $\mathbf{3 b}$ with the inherent contributions subtracted (left), Bulkiness rule for secondary alcohol geometry with the sign of the CD band E (middle), and experimental ECD spectra of 4, 5, and $\mathbf{6}$ (right) .....................................................

Figure S4. Chromatogram showing the chiral separation of a pair of epimers 5 and 6 .................7

Figure S5. Effects on B95-8 cells viabilities and inhibition on lytic EBV replication of 1-7 ........8

Figure S6. Results of microscale thermophoresis (MST) for 2GV9-compounds (1-7) interactions in vitro. . .9

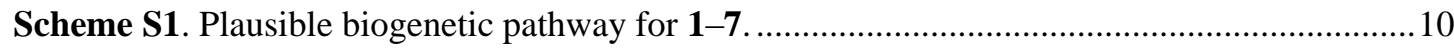

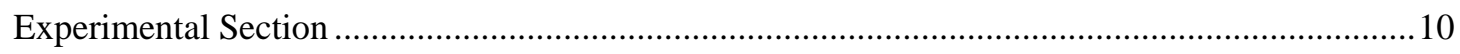

Experiment of in situ formed $\mathrm{Rh}_{2}\left(\mathrm{OCOCF}_{3}\right)_{4}-3 \mathbf{b}$ complex induced ECD spectrum .....................12

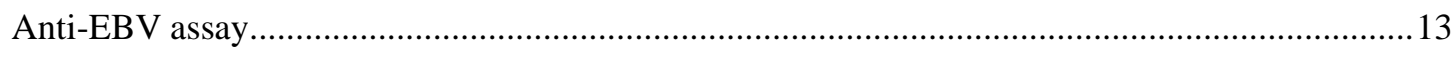

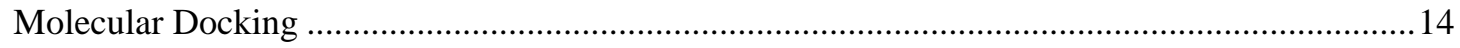

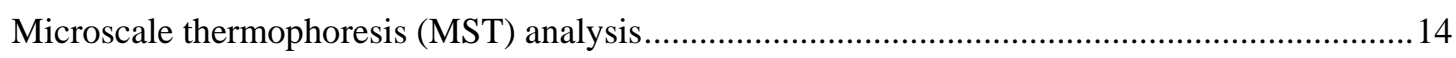

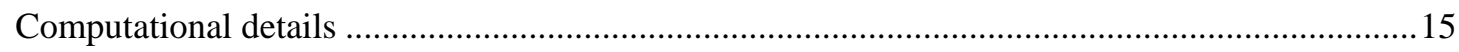

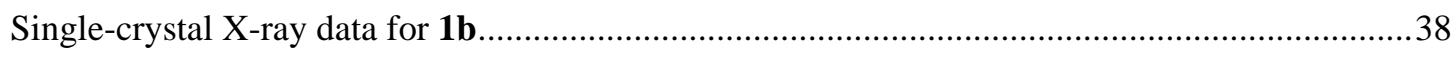

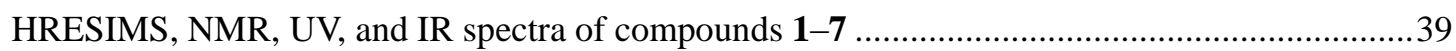


Table S1. ${ }^{1} \mathrm{H}$ NMR data of compounds 1-7 (600 MHz, in $\left.\mathrm{CDCl}_{3}\right)$.

\begin{tabular}{|c|c|c|c|c|c|c|c|}
\hline no. & $1 \mathrm{a} / \mathbf{1 b}$ & $2 a / 2 b$ & $\mathbf{3 a} / \mathbf{3} \mathbf{b}^{\mathrm{a}}$ & $4^{\mathrm{a}}$ & 5 & 6 & $7^{\text {a }}$ \\
\hline 2 & $1.78 \mathrm{~m}$ & $1.73 \mathrm{~m}$ & $1.77 \mathrm{~m}$ & $1.70 \mathrm{~m}$ & $1.70 \mathrm{~m}$ & $1.71 \mathrm{~m}$ & $1.47 \mathrm{~m}$ \\
\hline \multirow[t]{2}{*}{3} & $1.56 \mathrm{~m}$ & $2.19 \mathrm{~m}$ & $1.46 \mathrm{~m}$ & $1.49 \mathrm{dd}(13.0,8.6)$ & $1.49 \mathrm{~m}$ & $1.49 \mathrm{~m}$ & $0.90 \mathrm{~m}$ \\
\hline & $1.37 \mathrm{~m}$ & & $1.37 \mathrm{~d}(9.1)$ & $1.41 \mathrm{~d}(13.0)$ & & & $1.31 \mathrm{~m}$ \\
\hline 4 & $2.45 \mathrm{~m} ; 2.02 \mathrm{~m}$ & 5.12 br d $(9.2)$ & $1.81 \mathrm{~m} ; 1.25 \mathrm{~m}$ & $1.78 \mathrm{~m} ; 1.82 \mathrm{~m}$ & $1.81 \mathrm{~m} ; 1.73 \mathrm{~m}$ & $1.80 \mathrm{~m} ; 1.73 \mathrm{~m}$ & $1.56 \mathrm{~m} ; 1.52 \mathrm{~m}$ \\
\hline 5 & & & & $2.43 \mathrm{~m}$ & $2.44 \mathrm{~m}$ & $2.44 \mathrm{~m}$ & $2.66 \mathrm{~m}$ \\
\hline 6 & 3.87 br d (9.0) & $4.19 \mathrm{dd}(12.2,3.8)$ & $3.71 \mathrm{t}(9.0)$ & $2.84 \mathrm{~m}$ & $2.85 \mathrm{~m}$ & $2.85 \mathrm{~m}$ & $5.62 \mathrm{dd}(16.3,4.8)$ \\
\hline \multirow[t]{2}{*}{7} & $2.10 \mathrm{dd}(13.8,9.0)$ & $1.61 \mathrm{~d}(13.5,12.2)$ & $1.89 \mathrm{~m}$ & $3.02 \mathrm{dd}(7.8,2.6)$ & $3.01 \mathrm{dd}(7.9,2.5)$ & $3.02 \mathrm{dd}(7.8,2.3)$ & $5.52 \mathrm{dd}(16.3,7.2)$ \\
\hline & $1.61 \mathrm{~m}$ & $1.43 \mathrm{dd}(13.5,3.8)$ & $1.66 \mathrm{~m}$ & & & & \\
\hline 8 & & & $2.24 \operatorname{td}(11.4,5.5)$ & 1.35 br t (6.9) & $1.35 \mathrm{~m}$ & $1.35 \mathrm{~m}$ & $2.35 \mathrm{t}(7.2)$ \\
\hline 9 & 5.36 br d (15.8) & $5.24 \mathrm{~d}(15.6)$ & $1.85 \mathrm{~m}$ & $1.67 \mathrm{~m}$ & $1.67 \mathrm{~m}$ & $1.67 \mathrm{~m}$ & $1.93 \mathrm{~m} ; 1.59 \mathrm{~m}$ \\
\hline \multirow[t]{2}{*}{10} & 5.14 ddd $(15.8,10.0,4.0)$ & 4.97 ddd $(15.6,10.1,2.0)$ & $1.97 \mathrm{~d}(13.5)$ & $2.05 \mathrm{dt}(15.3,5.6)$ & $2.04 \mathrm{dt}(15.5,5.7)$ & $2.05 \mathrm{dt}(15.4,5.7)$ & $2.10 \mathrm{dt}(15.0,3.8)$ \\
\hline & & & $1.70 \mathrm{dd}(13.5,4.8)$ & $1.93 \mathrm{dd}(15.3,7.5)$ & $1.92 \mathrm{dt}(15.5,7.6)$ & $1.93 \mathrm{dt}(15.4,7.4)$ & $1.81 \mathrm{ddd}(15.0,10.2,4.5)$ \\
\hline \multirow[t]{2}{*}{11} & $2.48 \mathrm{dd}(14.2,4.0)$ & 2.72 br d (16.0) & & & & & \\
\hline & $2.37 \mathrm{dd}(14.2,10.0)$ & $2.31 \mathrm{dd}(16.0,10.1)$ & & & & & \\
\hline 12 & $1.09 \mathrm{~s}$ & $1.08 \mathrm{~s}$ & $4.74 \mathrm{~s} ; 4.70 \mathrm{~s}$ & $1.24 \mathrm{~s}$ & $1.24 \mathrm{~s}$ & $1.24 \mathrm{~s}$ & $1.22 \mathrm{~s}$ \\
\hline 13 & $1.01 \mathrm{~s}$ & $0.94 \mathrm{~s}$ & $1.64 \mathrm{~s}$ & $1.31 \mathrm{~s}$ & $1.31 \mathrm{~s}$ & $1.32 \mathrm{~s}$ & $1.23 \mathrm{~s}$ \\
\hline 14 & $5.19 \mathrm{~s} ; 4.93 \mathrm{~s}$ & $1.52 \mathrm{~s}$ & $0.79 \mathrm{~s}$ & $0.73 \mathrm{~d}(7.0)$ & $0.73 \mathrm{~d}(7.0)$ & $0.73 \mathrm{~d}(6.9)$ & $1.00 \mathrm{~d}(7.0)$ \\
\hline 15 & $1.07 \mathrm{~s}$ & $1.12 \mathrm{~s}$ & $1.10 \mathrm{~s}$ & $1.06 \mathrm{~s}$ & $1.07 \mathrm{~s}$ & $1.06 \mathrm{~s}$ & $1.09 \mathrm{~s}$ \\
\hline \multirow[t]{2}{*}{$7^{\prime}$} & $2.80 \mathrm{~m}$ & $2.61 \mathrm{dd}(16.7,5.3)$ & $2.43 \mathrm{dd}(16.4,4.9)$ & $2.86 \mathrm{~m}$ & $2.84 \mathrm{~m}$ & $2.86 \mathrm{~m}$ & $2.80 \mathrm{dd}(16.9,5.4)$ \\
\hline & $1.77 \mathrm{~m}$ & $2.06 \mathrm{dd}(16.7,13.1)$ & $2.02 \mathrm{dd}(16.4,12.0)$ & $1.82 \mathrm{~m}$ & $1.77 \mathrm{~m}$ & $1.79 \mathrm{~m}$ & $1.70 \mathrm{dd}(16.9,11.7)$ \\
\hline $9^{\prime}$ & 3.96 sept $(6.8)$ & 3.95 sept $(6.8)$ & 3.93 sept $(6.8)$ & 3.94 sept (6.7) & $3.82 \operatorname{sext}(6.8)$ & $3.82 \operatorname{sext}(6.8)$ & 3.69 sept $(6.8)$ \\
\hline $10^{\prime}$ & $1.12 \mathrm{~d}(6.8)$ & $1.12 \mathrm{~d}(6.8)$ & $1.10 \mathrm{~d}(6.8)$ & $1.12 \mathrm{~d}(6.7)$ & $1.71 \mathrm{~m} ; 1.38 \mathrm{~m}$ & $1.73 \mathrm{~m} ; 1.38 \mathrm{~m}$ & $1.18 \mathrm{~d}(6.8)$ \\
\hline $11^{\prime}$ & $1.14 \mathrm{~d}(6.8)$ & $1.13 \mathrm{~d}(6.8)$ & $1.12 \mathrm{~d}(6.8)$ & $1.13 \mathrm{~d}(6.7)$ & $0.88 \mathrm{t}(7.4)$ & $0.90 \mathrm{t}(7.4)$ & $1.16 \mathrm{~d}(6.8)$ \\
\hline $12^{\prime}$ & $1.35 \mathrm{~s}$ & $1.34 \mathrm{~s}$ & $1.27 \mathrm{~s}$ & $1.31 \mathrm{~s}$ & $1.12 \mathrm{~d}(6.8)$ & $1.10 \mathrm{~d}(6.8)$ & $1.36 \mathrm{~s}$ \\
\hline $13^{\prime}$ & $1.28 \mathrm{~s}$ & $1.27 \mathrm{~s}$ & $1.23 \mathrm{~s}$ & $1.27 \mathrm{~s}$ & $1.27 \mathrm{~s}$ & $1.27 \mathrm{~s}$ & $1.36 \mathrm{~s}$ \\
\hline $14^{\prime}$ & & & & & $1.31 \mathrm{~s}$ & $1.32 \mathrm{~s}$ & \\
\hline
\end{tabular}

${ }^{\mathrm{a}} 400 \mathrm{MHz}$. 
Table S2. ${ }^{13} \mathrm{C}$ NMR data of compounds 1-7 (150 MHz, in $\left.\mathrm{CDCl}_{3}\right)$.

\begin{tabular}{|c|c|c|c|c|c|c|c|}
\hline no. & $1 \mathrm{a} / \mathbf{1 b}$ & $2 \mathrm{a} / 2 \mathrm{~b}$ & $3 a / 3 b^{a}$ & $4^{a}$ & 5 & 6 & $7^{\mathrm{a}}$ \\
\hline 1 & 83.9 & 85.6 & 84.3 & 83.6 & 83.6 & 83.6 & 85.4 \\
\hline 2 & 37.5 & 38.6 & 40.7 & 37.1 & 37.1 & 37.0 & 35.8 \\
\hline 3 & 32.7 & 29.6 & 26.2 & 25.4 & 25.4 & 25.4 & 25.5 \\
\hline 4 & 32.2 & 130.4 & 41.35 & 30.1 & 30.1 & 30.1 & 31.2 \\
\hline 5 & 155.6 & 134.4 & 47.0 & 31.8 & 31.8 & 31.8 & 33.9 \\
\hline 6 & 73.0 & 76.4 & 82.3 & 61.1 & 61.1 & 61.2 & 136.7 \\
\hline 7 & 49.0 & 45.7 & 36.0 & 54.4 & 54.5 & 54.5 & 127.4 \\
\hline 8 & 36.0 & 35.64 & 47.1 & 48.8 & 48.8 & 48.8 & 49.7 \\
\hline 9 & 144.5 & 142.9 & 41.5 & 22.3 & 22.4 & 22.4 & 25.4 \\
\hline 10 & 120.1 & 119.6 & 39.3 & 32.8 & 32.8 & 32.8 & 32.3 \\
\hline 11 & 43.7 & 44.1 & 146.0 & 74.2 & 74.2 & 74.2 & 73.0 \\
\hline 12 & 24.7 & 22.9 & 111.2 & 28.6 & 28.6 & 28.6 & 28.2 \\
\hline 13 & 30.6 & 32.1 & 19.5 & 27.6 & 27.6 & 27.5 & 28.9 \\
\hline 14 & 113.8 & 11.0 & 12.4 & 11.1 & 11.1 & 11.1 & 16.6 \\
\hline 15 & 19.5 & 20.0 & 20.4 & 21.3 & 21.3 & 21.3 & 21.4 \\
\hline $1^{\prime}$ & 188.7 & 188.7 & 188.7 & 188.7 & 188.8 & 188.8 & 163.2 \\
\hline $2^{\prime}$ & 104.8 & 104.8 & 104.8 & 104.8 & 105.6 & 105.5 & 103.2 \\
\hline $3^{\prime}$ & 197.0 & 197.0 & 197.1 & 196.8 & 197.0 & 197.0 & 201.8 \\
\hline $4^{\prime}$ & 48.5 & 48.5 & 48.6 & 48.6 & 48.6 & 48.6 & 51.0 \\
\hline $5^{\prime}$ & 172.9 & 173.3 & 173.8 & 172.6 & 172.5 & 172.6 & 196.6 \\
\hline $6^{\prime}$ & 102.5 & 103.6 & 103.6 & 102.1 & 102.3 & 102.3 & 102.9 \\
\hline $7^{\prime}$ & 22.2 & 25.3 & 24.8 & 22.5 & 22.5 & 22.5 & 23.2 \\
\hline $8^{\prime}$ & 208.0 & 208.1 & 208.1 & 208.0 & 207.3 & 207.4 & 201.6 \\
\hline $9^{\prime}$ & 35.6 & 35.60 & 35.6 & 35.6 & 41.9 & 41.8 & 35.9 \\
\hline $10^{\prime}$ & 19.17 & 19.18 & 19.18 & 19.1 & 26.9 & 26.9 & 20.5 \\
\hline $11^{\prime}$ & 19.24 & 19.22 & 19.20 & 19.2 & 12.1 & 12.0 & 19.7 \\
\hline $12^{\prime}$ & 24.2 & 23.9 & 23.5 & 24.0 & 16.9 & 16.8 & 25.1 \\
\hline $13^{\prime}$ & 25.3 & 25.6 & 25.9 & 25.6 & 25.7 & 25.3 & 25.3 \\
\hline $14^{\prime}$ & & & & & 23.6 & 24.2 & \\
\hline
\end{tabular}

${ }^{\mathrm{a}} 100 \mathrm{MHz}$. 
Table S3. Predicted targets and their binding free energies with 1-7 (ICM docking scores).

\begin{tabular}{|c|c|c|c|c|c|c|c|c|c|c|c|}
\hline PDB ID & Protein name & 1a & $1 b$ & $\mathbf{2 a}$ & $2 b$ & $\mathbf{3 a}$ & $\mathbf{3 b}$ & 4 & 5 & 6 & 7 \\
\hline $1 \mathrm{CMV}$ & cytomegalovirus & $-61.33^{\mathrm{a}}$ & -89.43 & -102.3 & -90.85 & -30.41 & -129.09 & -80.23 & -106.92 & -44.40 & -88.23 \\
\hline 1PJR & DNA & -85.98 & -128.87 & -60.27 & -123.64 & -107.72 & -95.14 & -129.98 & -97.35 & -109.69 & -83.90 \\
\hline $1 \mathrm{WAJ}$ & DNA & -154.42 & -95.02 & -126.27 & -153.83 & -129.46 & -131.84 & -187.25 & -58.75 & -86.46 & -176.42 \\
\hline $2 \mathrm{GV} 9$ & DNA & -132.16 & -129.85 & -118.94 & -117.13 & -139.69 & -123.96 & -171.73 & -166.44 & -112.69 & -124.01 \\
\hline $3 \mathrm{H} 08$ & Ribonuclease & -50.80 & -45.27 & -55.16 & -37.04 & -98.47 & -91.08 & -87.47 & -40.52 & -66.34 & -53.08 \\
\hline $3 \mathrm{~T} 19$ & HIV-1 & -65.33 & -64.83 & -100.54 & -57.10 & -48.40 & -42.82 & -66.12 & -22.44 & -62.28 & -68.63 \\
\hline $4 \mathrm{U} 3 \mathrm{E}$ & Anaerobic & -99.24 & -94.11 & -51.22 & -109.38 & -90.92 & -81.28 & -114.98 & -96.14 & -123.97 & -163.51 \\
\hline $4 \mathrm{UXH}$ & Thymidine & -69.17 & -132.56 & -93.33 & -83.23 & -78.18 & -103.74 & -99.99 & -95.01 & -103.86 & -101.83 \\
\hline
\end{tabular}

${ }^{\mathrm{a}} \mathrm{mfscore}$ (Docking score of compounds with targets without depolarization et. al)

Table S4. Binding affinity and inhibitory activities of screened 1-7.

\begin{tabular}{ccc} 
Compound & $\begin{array}{c}\text { Dissociation constant with DNA } \\
\text { polymerase }(\mu \mathrm{M})\end{array}$ & $\mathrm{EC}_{50}$ \\
\hline $\mathbf{1 a}$ & $21.73 \pm 1.64$ & 10.33 \\
$\mathbf{1 b}$ & $64.50 \pm 4.78$ & 119.4 \\
$\mathbf{2 a}$ & $0.21 \pm 0.01$ & 0.57 \\
$\mathbf{2 b}$ & $20.51 \pm 2.11$ & 6.60 \\
$\mathbf{3 a}$ & n.b. & - \\
$\mathbf{3 b}$ & n.b. & - \\
$\mathbf{4}$ & $0.44 \pm 0.02$ & 0.49 \\
$\mathbf{5}$ & $12.30 \pm 5.88$ & 17.53 \\
$\mathbf{6}$ & $29.00 \pm 1.99$ & 14.47 \\
$\mathbf{7}$ & $89.52 \pm 3.94$ & $>300$ \\
\hline
\end{tabular}

n.b. is no clear binding detected in the MST measurement. 


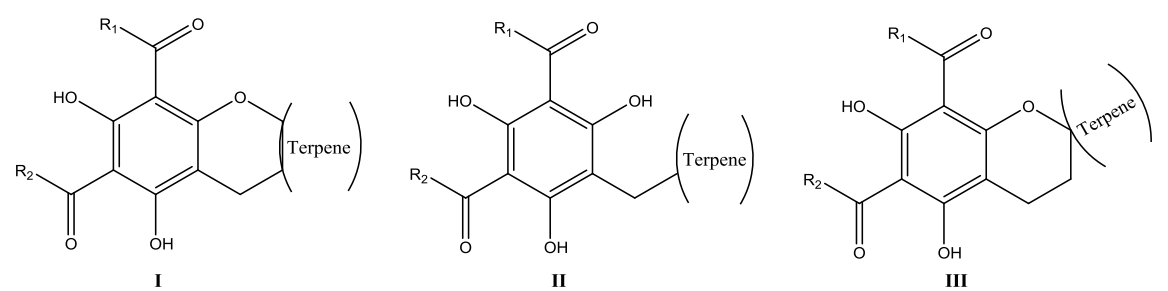<smiles>CC1=CC2C(CCC3(C)Oc4c(C=O)c(O)c(C=O)c(O)c4C(CC(C)C)C23C)C1</smiles><smiles>CC(C)CC1C2=C(OC(C3CC3(C)C)C1(C)CCC1OC1(C)C)C(C)(C)C(=O)C(C)(C)C2=O</smiles><smiles>C=C1CCC2C(c3ccccc3)c3c(O)c(C=O)c(O)c(C=O)c3OC2(C)CCC2C1CC2(C)C</smiles>
Org. Lett. 2010, 12, 656-659<smiles>CC(C)CC1c2c(O)c(C=O)c(O)c(C=O)c2OC2C3(C=C(C(C)C)CC(C)C3)CCC12C</smiles>

Tetrahedron Lett. 2012, 53, 2654-2658<smiles>C=C1CCC2(C)Oc3c(C=O)c(O)c(C=O)c(O)c3C(c3ccccc3)C2CCC2C1CC2(C)C</smiles>
Org. Lett. 2007, 9, 5135-5138<smiles>CC1=C2C3CC(C)(C(CC(C)C)c4c(O)c(C=O)c(O)c(C=O)c4O3)C2C2C(CC1)C2(C)C</smiles>

Eucalyptal D Tetrahedron Lett. 2012, 53, 2654-2658

Figure S1. Three types (I-III) of phloroglucinol-terpene adducts (First row) and the skeletons of phloroglucinol-sesquiterpene adducts previous reported. 

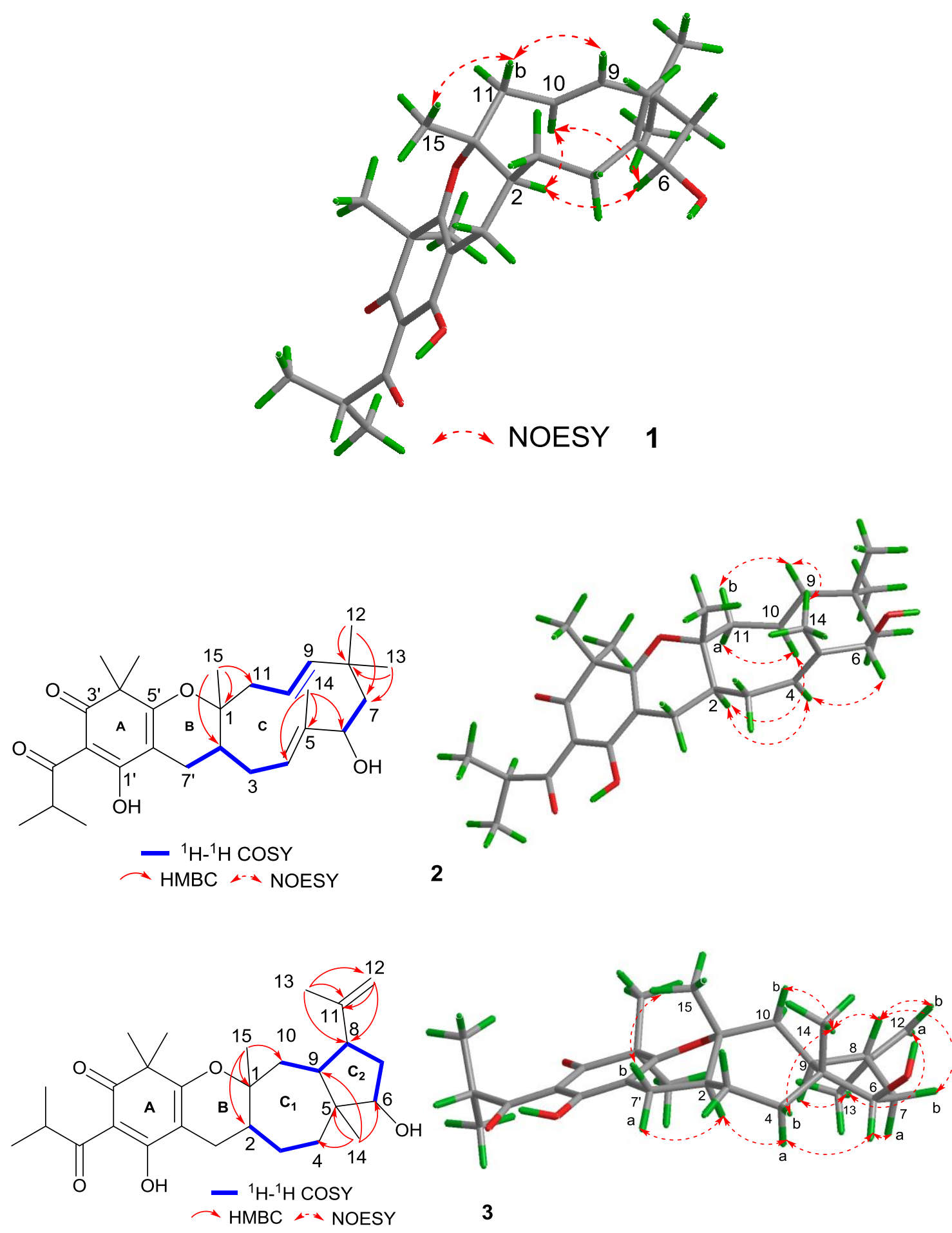


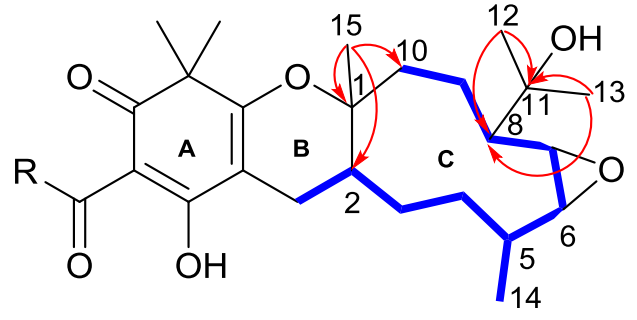

- ${ }^{1} \mathrm{H}-{ }^{1} \mathrm{H}$ COSY

$\sim$ HMBC $\cdots$ NOESY<smiles>[R]#[C+]C(C)C</smiles>

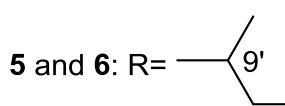

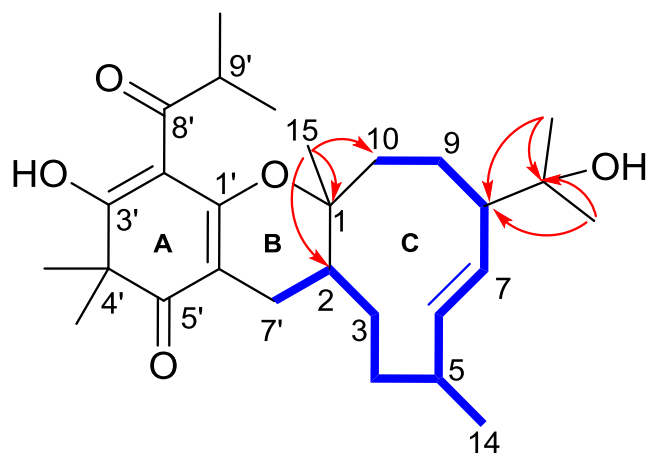

$-{ }^{1} \mathrm{H}-{ }^{1} \mathrm{H}$ COSY

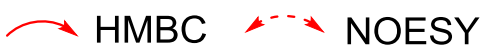

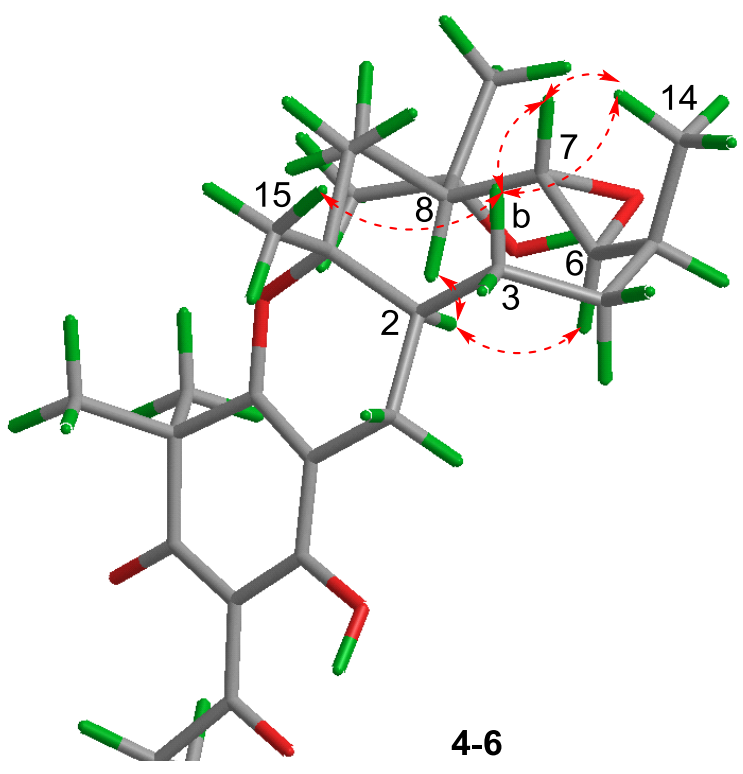

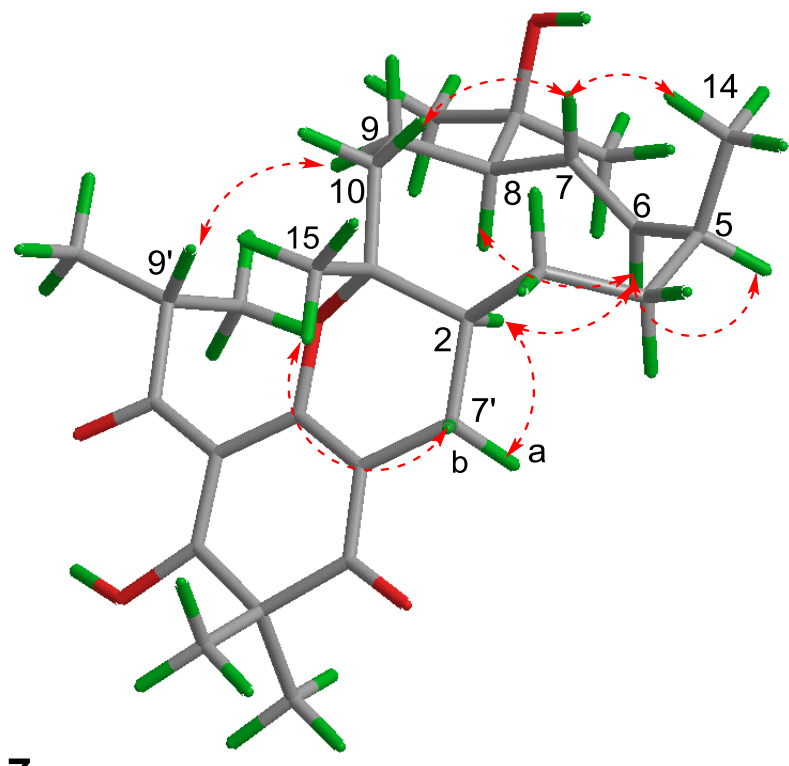

7

Figure S2. NOESY correlation of $\mathbf{1}$ and Key 2D NMR correlations of 2-7. 

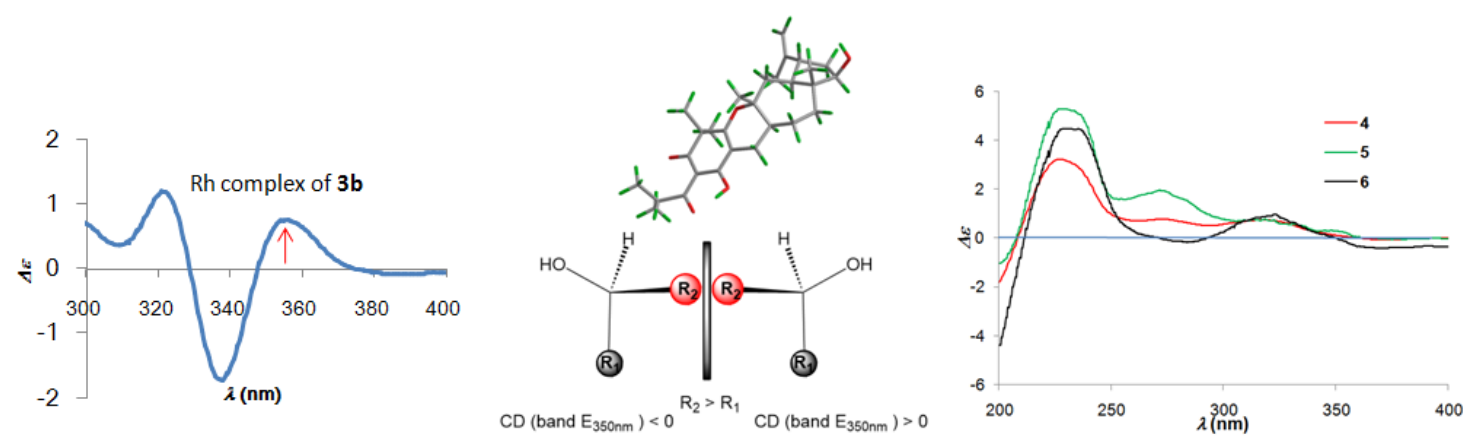

Figure S3. $\mathrm{Rh}_{2}\left(\mathrm{OCOCF}_{3}\right)_{4}$ induced ECD spectrum of $\mathbf{3 b}$ with the inherent contributions subtracted (left), Bulkiness rule for secondary alcohol geometry with the sign of the CD band E (middle), and experimental ECD spectra of $\mathbf{4 , 5}$, and $\mathbf{6}$ (right).

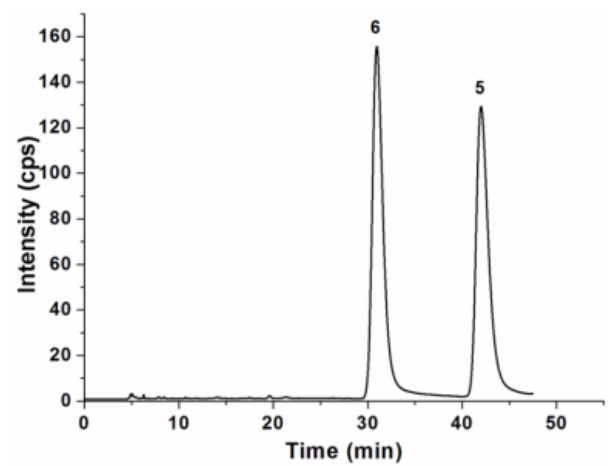

Figure S4. Chromatogram showing the chiral separation of a pair of epimers 5 and 6. The chromatographic conditions were using hexane/isopropanol (96/4 (V/V)) as mobile phase at a flow rate of $3.0 \mathrm{~mL} / \mathrm{min}$, a CHIRALPAK ${ }^{\circ} I C$ preparative column $(10 \times 250 \mathrm{~mm}, 5 \mu \mathrm{m}$ particles, Daicel, China) at room temperature and with UV detection at $245 \mathrm{~nm}$. Mass ratio of 5 and $\mathbf{6}$ was approximately 1:1. 

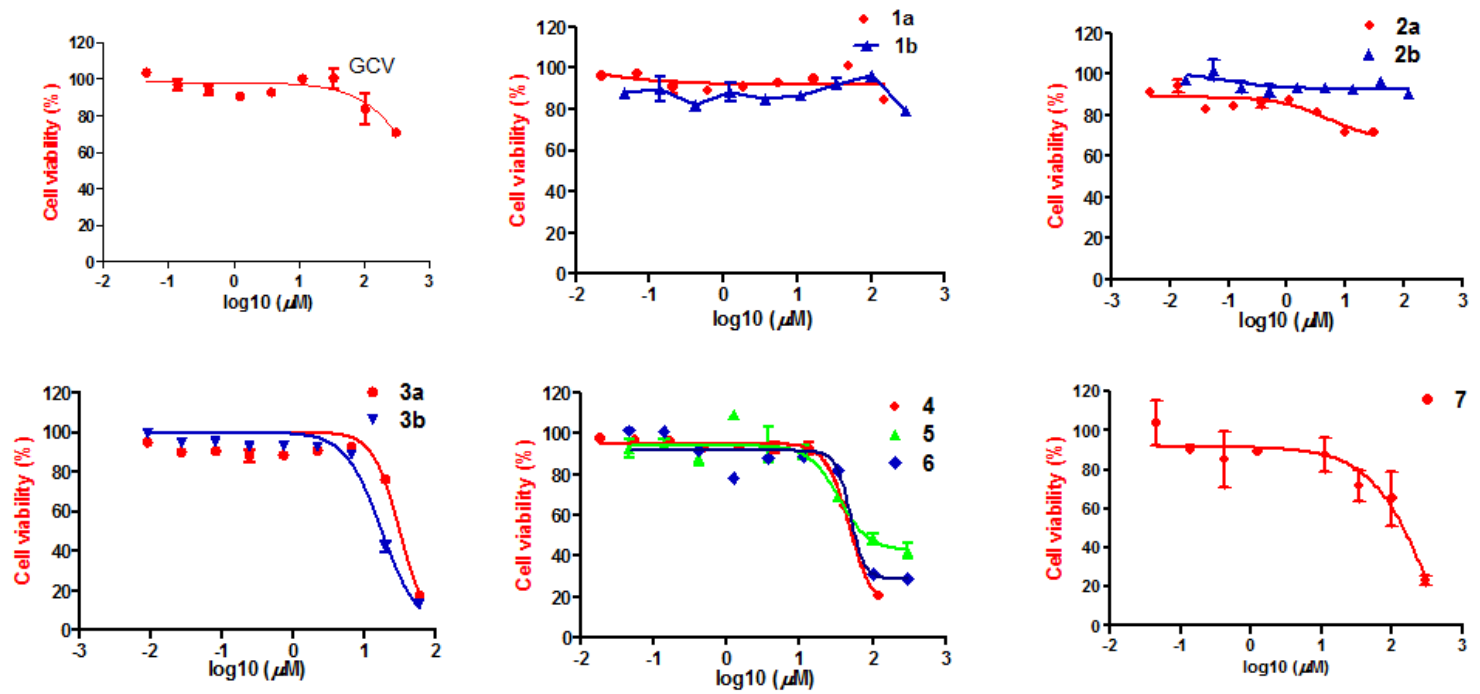

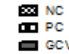

$\mathbf{g}^{\mathrm{NC}}$
$\mathbf{a}^{\mathrm{PC}}$

鱼
等
1b
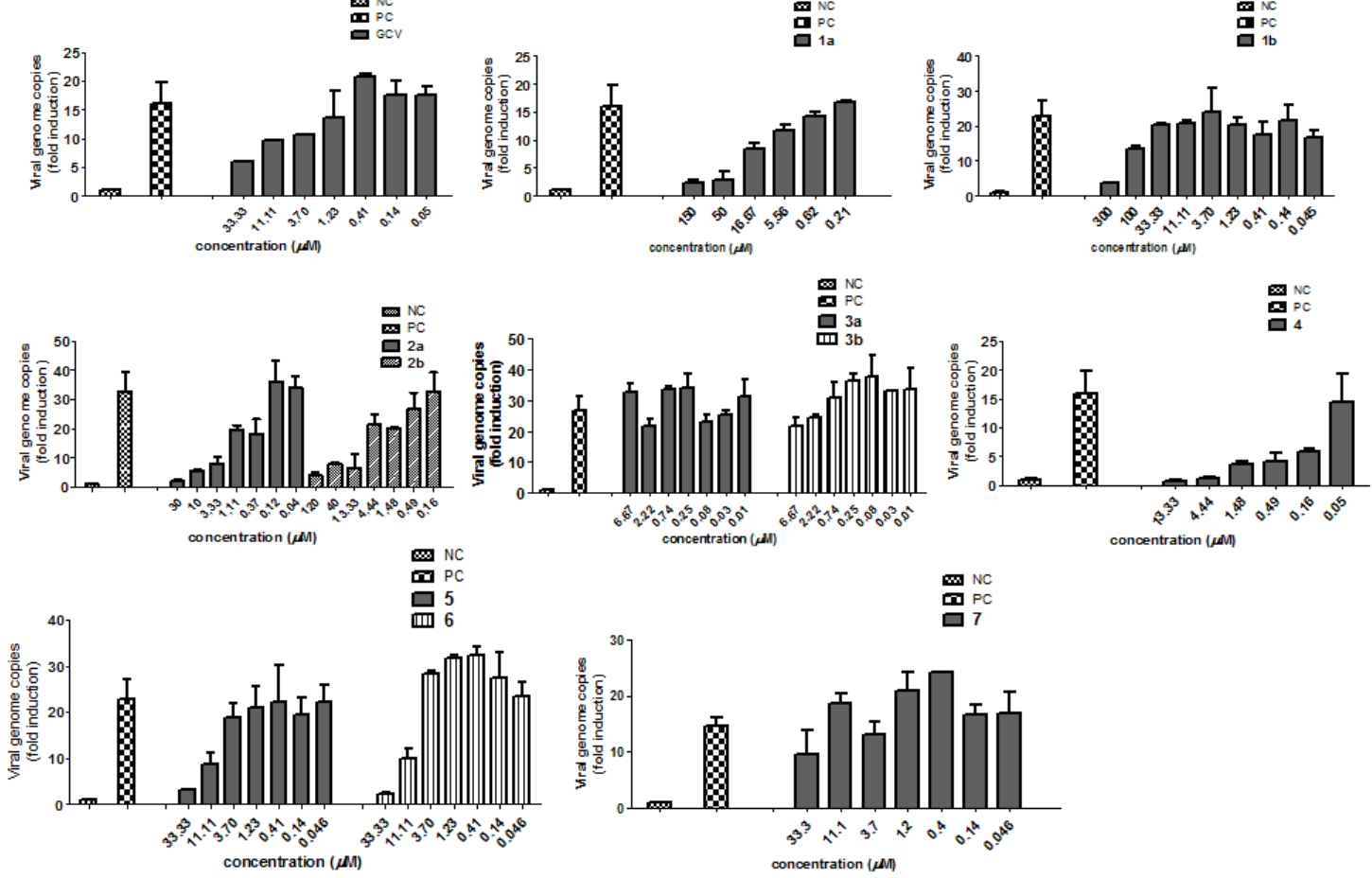

Figure S5. Effects on B95-8 cells viabilities and inhibition on lytic EBV replication of 1-7 were measured using GCV as positive control in vitro. B95-8 cells $\left(5 \times 10^{5} /\right.$ well) were treated with indicated concentrations of compounds in present of PMA. The 50\% cytotoxic concentration $\left(\mathrm{CC}_{50}\right)$ for each compound was calculated from these dose-response curves using Graphpad5.0 Prism. The $50 \%$ effective concentration $\left(\mathrm{EC}_{50}\right)$ values correspond to compound concentrations required to reduce quantitative expression of the copy number of intracellular viral genomic DNA by $50 \%$. Both results of $\mathrm{CC}_{50}$ and $\mathrm{EC}_{50}$ were presented as mean values with standard deviations $(\mathrm{n}=3)$. 

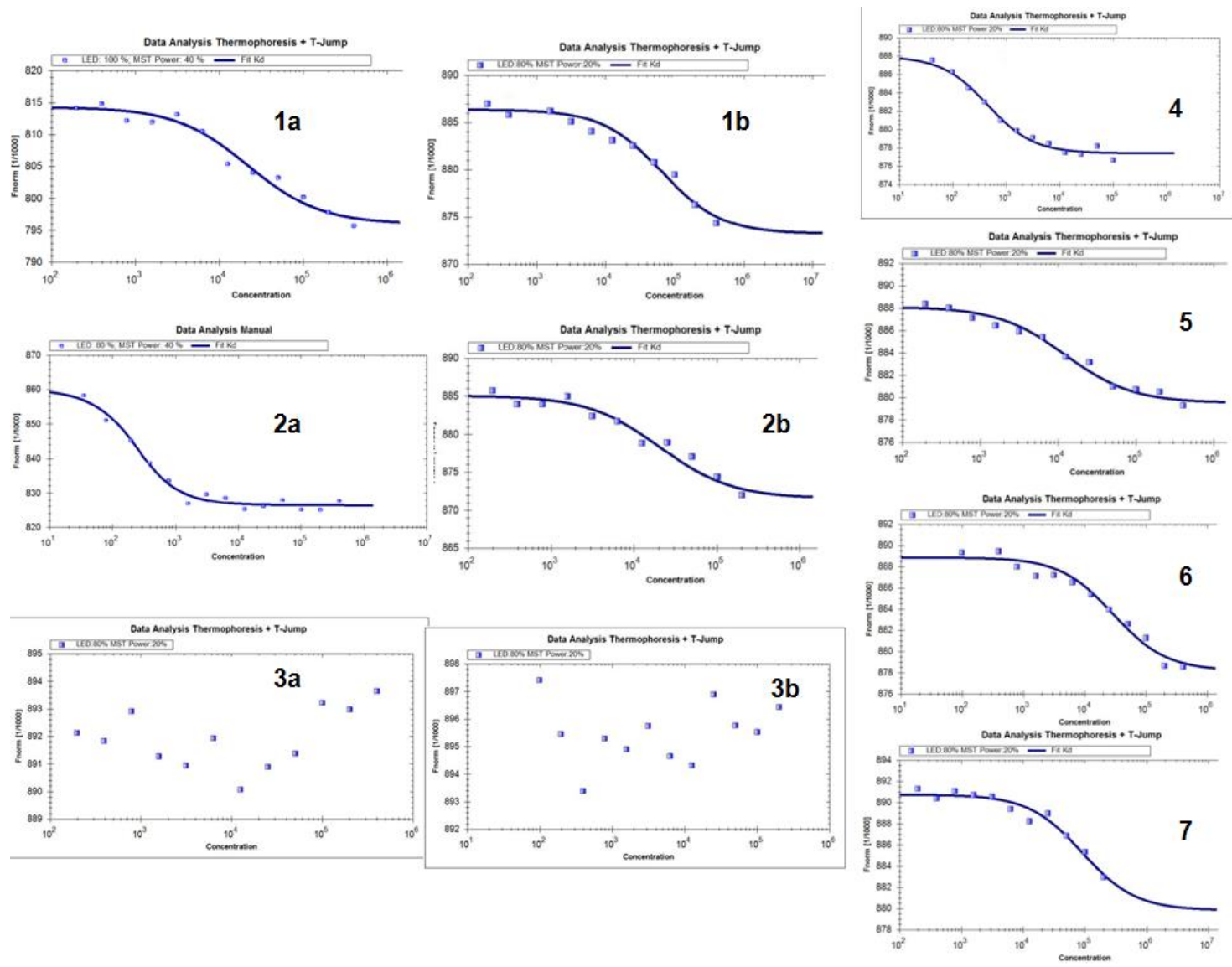

Figure S6. Results of microscale thermophoresis (MST) for 2GV9-compounds (1-7) interactions in vitro. 
Scheme S1. Plausible biogenetic pathway for 1-7.

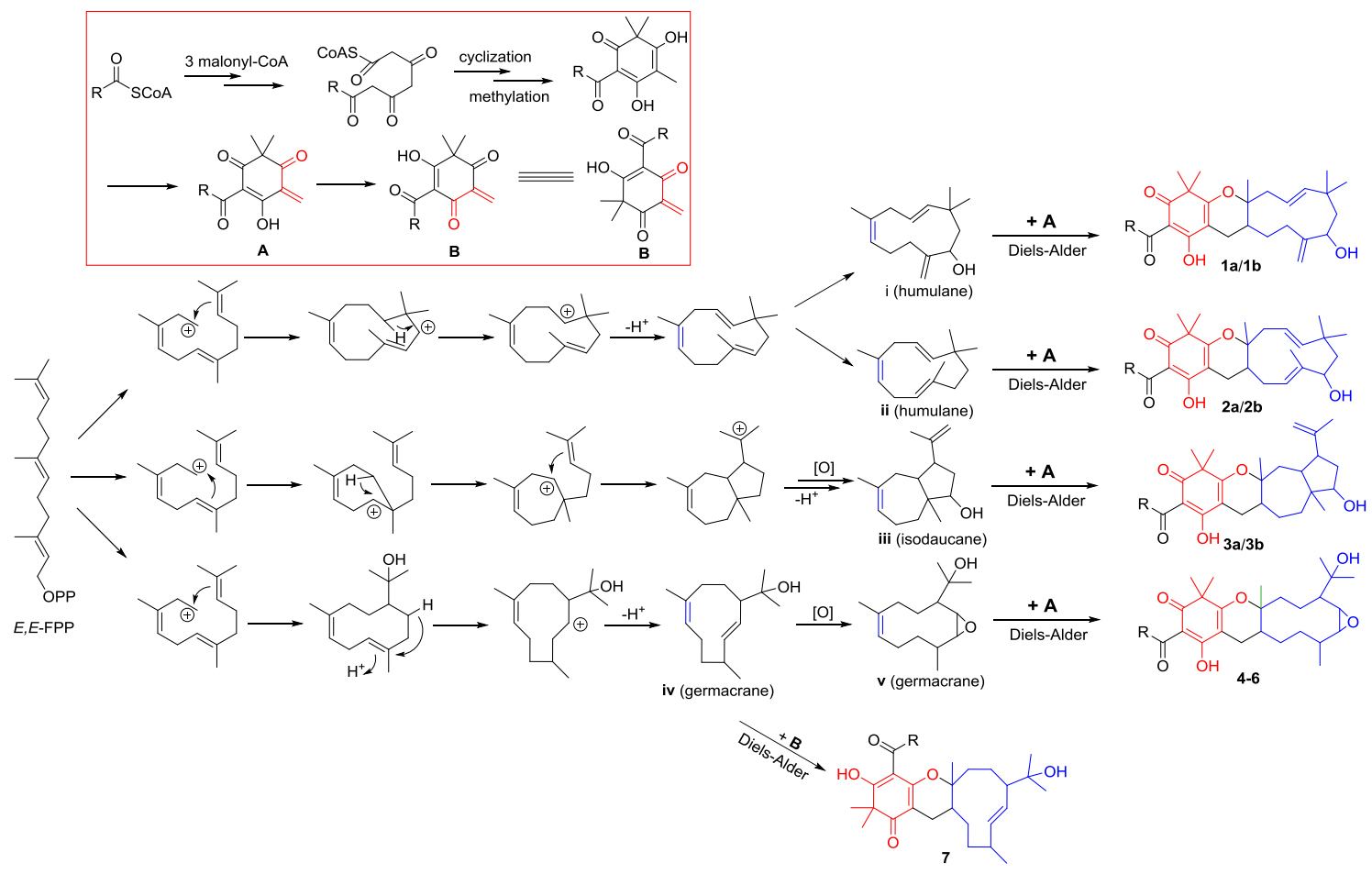

Hitherto, hyperjaponols A-G (1-7) were initially discovered with 6/6/11, 6/6/7/5, and 6/6/10 ring systems from $H$. japonicum. The basic chemical structures of these metabolites consist of filicinic acid moieties trans-fused with sesquiterpenoid units forming dihydropyran rings. The plausible biosynthetic route of compounds 1-7 could be proposed as shown in Scheme S1. At first, the acyledfilicinic acid moieties, including $\mathbf{A}$ and $\mathbf{B}$ were generated via the polyketide biosynthetic pathway involved three malonyl-CoA and an acyl-CoA. Then, sesquiterpenoid moieties (i-v) with skeletons of humulane, isodaucane, and germacrane were obtained from E,E-FPP through series of cyclization. Finally, key reactions of hetero-Diels-Alder cycloaddition lead to these unprecedented meroterpenoids 1-7.

\section{Experimental Section}

General methods: Extensive NMR data were recorded on a Bruker AM-600/400 spectrometer. HRESIMS data were characterized through a Thermo Fisher LC-LTQ-Orbitrap XL spectrometer. UV and FT-IR spectra were obtained via Varian Cary 50 and Bruker Vertex 70 apparatuses, respectively. A Perkin-Elmer 341 polarimeter was used for optical rotation measures. A Beijing Tech X-5 micro-melting point instrument was applied to measure melting points. Electronic circular dichroism (ECD) spectra were acquired by a JASCO J-810 spectrometer. The X-ray diffraction structural analysis were performed on a Bruker APEX DUO diffractometer using graphite monochromated $\mathrm{Cu} \mathrm{K} \alpha$ radiation $(\lambda=1.54178 \AA)$. HPLC were carried out with a LC 3050 Analysis of HPLC system (CXTH, Beijing, China) assembled with an UV 
3000 detector and a semi-preparative column $\left(5 \mu \mathrm{m}, 10 \times 250 \mathrm{~mm}, \mathrm{YMC}^{\circledR} \mathrm{XB}-\mathrm{C}_{18}\right)$. CHIRALPAK ${ }^{\circledR}$ IC column $(5 \mu \mathrm{m}, 10 \times 250 \mathrm{~mm}$, Daicel Chiral Technologies Co., Ltd. China) was rushed out for the enantioseparation. ODS (50 $\mu \mathrm{m}$, YMC Co. Ltd., Japan), Sephadex LH-20 (GE Healthcare Bio-Sciences AB, Sweden), and silica gel (200-300 mesh; Qingdao Bangkai High and New Technology Co. Ltd, Shandong, China) were applied as packing for column chromatography.

Plant material: The aerial parts of $H$. japonicum were collected from Da-Bie Mountain area of Hubei Province, P. R. China in October 2011, and identified by Professor Jianping Wang. A voucher specimen (ID 20111011) has been deposited with Herbarium of Materia Medica, School of Pharmacy, Tongji Medical College, Huazhong University of Science and Technology, P. R. China.

Extraction and isolation: The air-dried herbs of $H$. japonicum $(30 \mathrm{~kg}$ ) were soaked in $95 \% \mathrm{EtOH}$ at $25{ }^{\circ} \mathrm{C}$ to afford a crude extract $(0.75 \mathrm{~kg})$, which was suspended in $\mathrm{H}_{2} \mathrm{O}$ and partitioned successively with petroleum ether, $\mathrm{CHCl}_{3}$, and EtOAc. The petroleum ether-soluble portion $(300 \mathrm{~g})$ was subjected to silica gel column chromatography eluting with petroleum ether-acetone $(50 / 1-5 / 1)$ to yield seven fractions (Fr.1-Fr.7). On the basis of TLC analysis, Fr.4 was repeatedly chromatographed by silica gel column eluting with gradient petroleum ether in ethyl acetate to afford five subfractions (Fr.4.1-Fr.4.5). Fr.4.2 was further purified over MPLC (ODS, $\left.\mathrm{CH}_{3} \mathrm{OH}-\mathrm{H}_{2} \mathrm{O}, 50 \%-90 \%\right)$ to obtain subsections of Fr.4.2.1-Fr.4.2.4. Subsequently, Fr.4.2.2 was fractionated by Sephadex LH-20 $\left(\mathrm{CH}_{2} \mathrm{Cl}_{2}-\mathrm{MeOH}\right)$ and purified via semi-preparative HPLC $\left(\mathrm{CH}_{3} \mathrm{OH}-\mathrm{H}_{2} \mathrm{O} 85 \%\right)$ to yield hyperjaponols $\mathrm{D}-\mathrm{G}$, viz., 4-7 (4, $20 \mathrm{mg} ; 5$ and 6, $5 \mathrm{mg}$; 7, $6 \mathrm{mg}$ ), therein, 5 and $\mathbf{6}$ were mixtures of epimers. Meanwhile, Fr.4.2.3 was applied on silica gel $\mathrm{CC}\left(\mathrm{CHCl}_{3}-\mathrm{CH}_{3} \mathrm{OH} 35: 1\right)$, Sephadex $\mathrm{LH}-20\left(\mathrm{CH}_{2} \mathrm{Cl}_{2}-\mathrm{MeOH}, 1: 1\right)$, and semi-preparative $\mathrm{HPLC}\left(\mathrm{CH}_{3} \mathrm{CN}-\mathrm{H}_{2} \mathrm{O} 75 \%\right)$ to provide three pairs of racemates hyperjaponols A-C, viz., 1 (4 mg), 2 (3.5 mg), and 3 (10 mg).

Semipreparative enantioseparation: To achieve the preparative separations of three racemic mixture pairs $(\mathbf{1}-\mathbf{3})$ and a pair of epimers (5 and $\mathbf{6})$, the analytical and semipreparative enantioseparation were subjected through chiral HPLC methods. The separation chromatograms of the racemates $(\mathbf{1}-\mathbf{3})$ and the pair of epimers $(\mathbf{5}$ and $\mathbf{6})$ were shown in Figure 2 and Figure S4, SI, respectively. Mass ratios of optical antipodes in each isolated enantiomeric fraction (1-3) were approximately of 1:1, and interestingly, the same phenomenon also presented in the epimers pair (5 and 6) (Figure S4). In addition, better than baseline separation were obtained of 1-3 and 5/6 from their racemic mixtures or epimerism mixture based on the enantiomer (Figure 2) or epimer peaks (Figure S4) to noise ratio. The chromatographic conditions for $\mathbf{1}-\mathbf{3}$ and $\mathbf{5 / 6}$ were using hexane/isopropanol (95/5 (V/V) and 96/4 (V/V) for 1-3 and 5-6, respectively.) as mobile phase at a flow rate of $3.0 \mathrm{~mL} / \mathrm{min}$, a CHIRALPAK ${ }^{\circledR} \mathrm{IC}$ preparative column $(10$ $\times 250 \mathrm{~mm}, 5 \mu \mathrm{m}$ particles, Daicel, China) column at room temperature, and with UV detection at $245 \mathrm{~nm}$.

Compound 1: UV $\left(\mathrm{CH}_{3} \mathrm{OH}\right) \lambda_{\max }(\log \varepsilon)=224$ (3.84), 244 (3.85), 327 (3.91) nm; IR $v_{\max }=$ $3411,2958,2930,2869,1658,1624,1524,1473,1382 \mathrm{~cm}^{-1}$; for ${ }^{1} \mathrm{H}$ NMR (600 MHz) and ${ }^{13} \mathrm{C} \mathrm{NMR}$ 
(150 MHz) data see Tables S1 and S2; HRESIMS $[\mathrm{M}+\mathrm{H}]^{+} \mathrm{m} / z \quad 457.2944$ (calcd for $\left.\mathrm{C}_{28} \mathrm{H}_{41} \mathrm{O}_{5}, 457.2954\right)$.

1a: Orange oil, $[\alpha]_{\mathrm{D}}^{20}+102.0\left(c 0.07, \mathrm{CH}_{3} \mathrm{OH}\right) ; \mathrm{ECD}\left(\mathrm{CH}_{3} \mathrm{OH}\right) \lambda(\Delta \varepsilon) 203(+2.71), 229(+2.94)$ nm.

1b: Colorless crystal, mp $=175.5-178.0^{\circ} \mathrm{C},[\alpha]_{\mathrm{D}}^{20}-103.3\left(c 0.07, \mathrm{CH}_{3} \mathrm{OH}\right) ; \mathrm{ECD}\left(\mathrm{CH}_{3} \mathrm{OH}\right), \lambda$ (4ع) $201(-3.30), 229(-3.25) \mathrm{nm}$.

Compound 2: UV $\left(\mathrm{CH}_{3} \mathrm{OH}\right) \lambda_{\max }(\log \varepsilon)=227$ (3.22), 242 (3.25), 325 (3.28) nm; IR $v_{\max }=$ $3460,2963,2930,1658,1624,1553,1474,1383 \mathrm{~cm}^{-1}$; for ${ }^{1} \mathrm{H}$ NMR (600 MHz) and ${ }^{13} \mathrm{C}$ NMR (150 $\mathrm{MHz}$ ) data see Tables $\mathrm{S} 1$ and S2; HRESIMS $[\mathrm{M}+\mathrm{H}]^{+} \mathrm{m} / z 457.2946$ (calcd for $\mathrm{C}_{28} \mathrm{H}_{41} \mathrm{O}_{5}, 457.2954$ ).

2a: Orange oil, $[\alpha]_{\mathrm{D}}^{20}+135.4\left(c 0.03, \mathrm{CH}_{3} \mathrm{OH}\right)$; $\mathrm{ECD}\left(\mathrm{CH}_{3} \mathrm{OH}\right) \lambda(\Delta \varepsilon) 216(+13.01) \mathrm{nm}$.

2b: Lightsalmon oil, $[\alpha]_{\mathrm{D}}^{20}-134.5\left(c 0.03, \mathrm{CH}_{3} \mathrm{OH}\right) ; \mathrm{ECD}\left(\mathrm{CH}_{3} \mathrm{OH}\right) \lambda(\Delta \varepsilon) 217(-10.91) \mathrm{nm}$.

Compound 3: UV $\left(\mathrm{CH}_{3} \mathrm{OH}\right) \lambda_{\max }(\log \varepsilon)=226$ (4.29), 241 (4.33), 327 (4.11) nm; IR $v_{\max }=$ 3432, 3074, 2971, 2933, 2872, 1652, 1613, 1523, 1472, $1381 \mathrm{~cm}^{-1}$; for ${ }^{1} \mathrm{H}$ NMR (400 MHz) and ${ }^{13} \mathrm{C}$ NMR (100 MHz) data see Ss 1 and S2; HRESIMS $[\mathrm{M}+\mathrm{H}]^{+} \mathrm{m} / z 457.2941$ (calcd for $\mathrm{C}_{28} \mathrm{H}_{41} \mathrm{O}_{5}$, 457.2954).

3a: Orange-red oil, $[\alpha]_{\mathrm{D}}^{20}+122.0\left(c 0.15, \mathrm{CH}_{3} \mathrm{OH}\right)$; $\mathrm{ECD}\left(\mathrm{CH}_{3} \mathrm{OH}\right) \lambda(\Delta \varepsilon) 231(+2.35), 277$ $(+1.58), 320(+1.28) \mathrm{nm}$.

3b: Light pink oil, $[\alpha]_{\mathrm{D}}^{20}-121.8\left(c 0.11, \mathrm{CH}_{3} \mathrm{OH}\right)$; $\mathrm{ECD}\left(\mathrm{CH}_{3} \mathrm{OH}\right) \lambda(\Delta \varepsilon) 232(-2.63), 273$ $(-1.29), 321(-1.53) \mathrm{nm}$.

Compound 4: bright-yellow oil, $[\alpha]_{\mathrm{D}}^{20}+64.7\left(c\right.$ 0.11, $\left.\mathrm{CH}_{3} \mathrm{OH}\right)$; $\mathrm{ECD}\left(\mathrm{CH}_{3} \mathrm{OH}\right) \lambda(\Delta \varepsilon) 227$ (+3.23), 272 (+0.78), $317(+0.74) \mathrm{nm} ; \mathrm{UV}\left(\mathrm{CH}_{3} \mathrm{OH}\right) \lambda_{\max }(\log \varepsilon)=229$ (3.64), 240 (3.15), 329 (3.26) $\mathrm{nm}$; IR $v_{\max }=3521,3054,2980,2933,2873,1659,1626,1475,1383 \mathrm{~cm}^{-1}$; for ${ }^{1} \mathrm{H}$ NMR $(400 \mathrm{MHz})$ and ${ }^{13} \mathrm{C}$ NMR (100 MHz) data see Tables S1 and S2; HRESIMS [M + H] ${ }^{+} \mathrm{m} / \mathrm{z} 475.3046$ (calcd for $\left.\mathrm{C}_{28} \mathrm{H}_{43} \mathrm{O}_{6}, 475.3060\right)$.

Compound 5: orange-red oil, $[\alpha]_{\mathrm{D}}^{20}+116.0\left(c 0.05, \mathrm{CH}_{3} \mathrm{OH}\right)$; $\mathrm{ECD}\left(\mathrm{CH}_{3} \mathrm{OH}\right) \lambda(\Delta \varepsilon) 227$ (+5.28), $271(+1.95), 318(+0.76) \mathrm{nm}$; UV $\left(\mathrm{CH}_{3} \mathrm{OH}\right) \lambda_{\max }(\log \varepsilon)=227$ (3.99), 242 (3.97), 327 (4.07) $\mathrm{nm}$; IR $v_{\max }=3488,2970,2934,1659,1624,1524,1470 \mathrm{~cm}^{-1}$; for ${ }^{1} \mathrm{H}$ NMR $(600 \mathrm{MHz})$ and ${ }^{13} \mathrm{C}$ NMR (150 MHz) data see Tables S1 and S2; HRESIMS $[\mathrm{M}+\mathrm{H}]^{+} \mathrm{m} / z 489.3205$ (calcd for $\mathrm{C}_{29} \mathrm{H}_{45} \mathrm{O}_{6}$, 489.3216).

Compound 6: light pink oil, $[\alpha]_{\mathrm{D}}^{20}+65.0,\left(c 0.04, \mathrm{CH}_{3} \mathrm{OH}\right)$; $\mathrm{ECD}\left(\mathrm{CH}_{3} \mathrm{OH}\right) \lambda(\Delta \varepsilon) 229(+4.48)$, $283(-0.17), 323(+0.96) \mathrm{nm}$; UV $\left(\mathrm{CH}_{3} \mathrm{OH}\right) \lambda_{\max }(\log \varepsilon)=229$ (3.75), 240 (3.78), 320 (3.72) nm; IR $v_{\max }=3435,2959,2926,1659,1622,1525,1464 \mathrm{~cm}^{-1}$; for ${ }^{1} \mathrm{H}$ NMR $(600 \mathrm{MHz})$ and ${ }^{13} \mathrm{C}$ NMR $(150$ MHz) data see Tables S1 and S2; HRESIMS [M + H] ${ }^{+} \mathrm{m} / z$, 489.3208 (calcd for $\mathrm{C}_{29} \mathrm{H}_{45} \mathrm{O}_{6}, 489.3216$ ).

Compound 7: violet oil, $[\alpha]_{\mathrm{D}}^{20}+46.2\left(c 0.14, \mathrm{CH}_{3} \mathrm{OH}\right) ; \mathrm{ECD}\left(\mathrm{CH}_{3} \mathrm{OH}\right) \lambda(\Delta \varepsilon) 226(+13.74), 278$ (+1.75), $317(+3.74) \mathrm{nm} ; \mathrm{UV}\left(\mathrm{CH}_{3} \mathrm{OH}\right) \lambda_{\max }(\log \varepsilon)=223(4.06), 311(4.14) \mathrm{nm} ; \mathrm{IR} v_{\max }=3435,2959$, 2926, 1659, 1622, 1525, $1464 \mathrm{~cm}^{-1}$; for ${ }^{1} \mathrm{H}$ NMR (400 MHz) and ${ }^{13} \mathrm{C}$ NMR (100 MHz) data see Tables S1 and S2; HRESIMS [M+ H] ${ }^{+} \mathrm{m} / z 459.3097$ (calcd for $\mathrm{C}_{28} \mathrm{H}_{43} \mathrm{O}_{5}, 459.3110$ ).

\section{Experiment of in situ formed $\mathrm{Rh}_{2}\left(\mathrm{OCOCF}_{3}\right)_{4}-3 b$ complex induced ECD spectrum}

Based on the reported method, ${ }^{1}$ an experiment of in situ formed $\mathrm{Rh}_{2}\left(\mathrm{OCOCF}_{3}\right)_{4}$ complex induced ECD spectrum was implemented to determine the absolute configuration of the C-6 secondary alcohol in $\mathbf{3 b}$. The 1:2 mixture of $\mathbf{3 b}-\mathrm{Rh}_{2}\left(\mathrm{OCOCF}_{3}\right)_{4}$ was dissolved in anhydrous $\mathrm{CH}_{2} \mathrm{Cl}_{2}$ at approximately concentration of $1 \mathrm{mg} / \mathrm{mL}$. The in situ formed $\left[\mathrm{Rh}_{2}\left(\mathrm{OCOCF}_{3}\right)_{4}\right]$ complex was measured its first $\mathrm{CD}$ spectrum immediately 
after blending, and its time evolution was monitored until stationary (ca. 10 min after blending). After subtracted its inherent CD contribution, the observed Cotton effects of the E band at ca. $350 \mathrm{~nm}$ (Figure S3, left) within the induced CD spectrum was correlated to the chiral configuration of the C-6 secondary alcohol moiety.

The $\left[\mathrm{Rh}_{2}\left(\mathrm{OCOCF}_{3}\right)_{4}\right]$ complex of $\mathbf{3 b}$ afforded a positive sign of the $\mathrm{E}$ band at ca. $350 \mathrm{~nm}$ (Figure S3, left), which was unambiguously correlated with the $6 \mathrm{~S}$ configuration by employing the bulkiness rule (Figure S3, middle). ${ }^{1}$ Consequently, the stereostructure of $\mathbf{3 b}$ was established as $1 S, 2 R, 5 S, 6 S, 8 S, 9 R$.

1. (a) Frelek, J.; Szczepek, W. J. Tetrahedron Asymmetr. 1999, 10, 1507-1520; (b) Luo, J.; Liu, Xi.; Li, E.; Guo, L.; Che, Y. J. Nat. Prod. 2013, 76, 107-112; (c) He, J. W.; Qin, D. P.; Gao, H.; Kuang, R. Qi.; Yu, Y.; Liu, X. Z.; Yao, X. S. Molecules 2014, 19, 20880-20887.

\section{Anti-EBV assay}

B95-8 cells were used to determine the antiviral activity of compounds 1-7 against EBV. For the lytic replication of EBV, B95-8 cells were induced with $20 \mathrm{ng} / \mathrm{mL}$ 12-O-tetradecanoyl-phorbol-13-acetate (PMA/TPA) (Beyotime). B95-8 cells $\left(5 \times 10^{5} /\right.$ well $)$ were treated with indicated concentrations of compounds in present of TPA. Then cell viability was assessed at $60 \mathrm{~h}$ post drugs-induction by AlamarBlue ${ }^{\circledR}$ Cell Viability Assay (Thermo Fisher Scientific, Waltham, Massachusetts, USA) according to the manufacturer's protocol. The luminescent signal was measured using the Envison 2102 Multilabel Reader (Perkin Elmer, Waltham, MA, USA). The 50\% cytotoxic concentration $\left(\mathrm{CC}_{50}\right)$ for each compound was calculated from these dose-response curves using Graphpad5.0 Prism. The results were expressed as the mean values of three replicate experiments and standard deviation.

The antiviral effect of compounds on EBV was determined by qPCR assay. EBV genomic DNA was isolated as previously described. ${ }^{1}$ The copy numbers of viral DNA were measured by qPCR. All qPCR assays were performed using a Bio-Rad CFX96 Touch $^{\mathrm{TM}}$ Real-Time PCR detection system using the iTaq $^{\mathrm{TM}}$ Universal SYBR ${ }^{\circledR}$ Green Supermix (Bio-Rad) with primers directed to the EBNA1 gene (forward, 5'GCCGGTGTGTTCGTATATGG - - $3^{\prime}$ and reverse, 5'-CAAAACCTCAGCAAATATATGAG -3'). ${ }^{2}$ The intracellular viral genomic DNA in each sample was normalized to the amount of the GAPDH gene also determined by qPCR by using primers (forward, 5'-GCTCCCTCTTTCTTTGCAGCAAT-3' and reverse, 5'-TACCATGAGTCCTTCCACGATAC- 3 '). ${ }^{3}$ The $50 \%$ effective concentration values $\left(\mathrm{EC}_{50}\right)$ correspond to compound concentrations required to reduce quantitative expression of the copy number of intracellular viral genomic DNA by $50 \%$. The results were expressed as the mean values of three replicate experiments and standard deviation.

1. A. Wiedmer, P. Wang, J. Zhou, A. J. Rennekamp, V. Tiranti, M. Zeviani, P. M. Lieberman, J. Virol. 2008, 82, 4647-4655.

2. S. J. Stevens, M. B. Vervoort, A. J. van den Brule, P. L. Meenhorst, C. J. Meijer, J. M. Middeldorp, J. Clin. Microbiol. 1999, 37, 2852-2857. 


\section{Molecular Docking}

For the small scale inverse docking, ${ }^{1}$ crystal structures of docking targets (Table S3) were obtained from the Protein Data Bank (http://www.rcsb.org). ${ }^{2}$ The docking was performed by using ICM 3.8.2 modeling software on an Intel i7 4960 processor (MolSoft LLC, San Diego, CA). ${ }^{3}$ Ligand binding pocket residues were selected by using graphical tools in the ICM software, to create the boundaries of the docking search. In the docking calculation, potential energy maps of the receptor were calculated using default parameters. Compounds were imported into ICM and an index file was created. Conformational sampling was based on the Monte Carlo procedure, ${ }^{4}$ and finally the lowest-energy and the most favourable orientation of the ligand were selected.

1. (a) S. D. Guptaa, M. K. Bommaka, G. I. Mazaira, M. D. Galigniana, C. V. S. Subrahmanyam, N. L. Gowrishankar, N. M. Raghavendra, Int. J. Biol. Macromol. 2015, 80, 253-259; (b) W. Hong, Y. Wang, Z. Chang, Y. Yang, J. Pu, T. Sun, S. Kaur, J. C. Sacchettini, H. Jung, W. Lin Wong, L. Fah Yap, Y. Fong Ngeow, I. C. Paterson, H. Wang, Sci. Rep. 2015, 5, doi: 10.1038/srep15328.

2. (a) H. S. Shieh, R. G. Kurumbail, A. M. Stevens, R. A Stegeman, E. J. Sturman, J. Y. Pak, A. J. Wittwer, M. O. Palmier, R. C. Wiegand, B. C. Holwerda, W. C. Stallings, Nature 1996, 383, 279-282; (b) H. S. Subramanya, L. E. Bird, J. A. Brannigan, D. B. Wigley, Nature 1996, 384 , 379-383; (c) S. Liu, J. D. Knafels, J. S.Chang, G. A. Waszak, E. T. Baldwin, M. R. J. Deibel, D. R. Thomsen, F. L. Homa, P. A. Wells, M. C. Tory, R. A. Poorman, H. Gao, X. Qiu, A. P. Seddon, J. Bio. Chem. 2006, 281, 18193-18200; (d) R. Kathleen, C. Jacob, M. Susan, Biochemistry 2009, 48, 5890-5898; (e) R. Gomez, S. J. Jolly, T. Williams, J. P. Vacca, M. Torrent, G. McGaughey, M. T. Lai, P. Felock, V. Munshi, D. Distefano, J. Flynn, M. Miller, Y. Yan, J. Reid, R. Sanchez, Y. Liang, B. Paton, B. L. Wan, N. Anthony, J. Med. Chem. 2013, 54, 7920-7933; (f) Y. Wei, M. A. Funk, L. A. Rosado, J. Baek, C. L. Drennan, J. Stubbe, P. Natl. Acad. Sci. USA. 2014, 111, E3756-E3765; (g) J. Timm, C. Bosch Navarrete, E. Recio, J. E. Nettleship, H. Rada, D. González Pacanowska, K. S. Wilson, Plos Negl. Trop. Dis. 2015, 9, doi: 10.1371/journal.pntd.0003781.

3. (a) R. Abagyan, M. Totrov, D. Kuznetsov, J. Comput. Chem. 1994, 15, 488-506; (b) Z. Li, H. A. Scheraga, P. Natl. Acad. Sci. USA. 1987, 84, 6611-6615.

4. Z. Li, H. A. Scheraga. Monte Carlo-minimization approach to the multiple-minima problem in protein folding. Proc. Natl. Acad. Sci. USA. 1987, 84, 6611-6615.

\section{Microscale thermophoresis (MST) analysis for interactions between compounds with DNA polymerase}

The DNA polymerase-compound interactions were quantified with microscale thermophoresis (MST) method. ${ }^{1}$ MST measurements were performed on a Monolith NT 115 (NanoTemper Technologies, Germany). Recombinant DNA polymerase beta (ProSpec-Tany, Israel) was first re-buffered in the labelling buffer to a final concentration of $10 \mu \mathrm{M}$. NT647 was added in twofold molar excess and incubated for $30 \mathrm{~min}$ at room temperature. Finally, the labelled protein was separated from excess dye 
by a gravity flow column for the MST measurements. Series compounds were diluted in 12 dilution steps covering the range from $400 \mu \mathrm{M}$ to $190 \mathrm{nM}$. Then $10 \mu \mathrm{L}$ of labelled protein was added to $10 \mu \mathrm{L}$ sample solution. The samples were loaded in NT hydrophobic capillaries and measured with $100 \%$ LED and $40 \%$ laser power, with a laser on-rate of $30 \mathrm{~s}$ and a laser off-rate of $5 s$ in triplicates. The $\mathrm{K}_{\mathrm{d}}$ values and results were calculated by the NanoTemper software version 1.0.1 (Table S4 and Figure S6).

1. O. Lange, C. Wolf, J. Dietze, J. Elsaesser, R. Morbitzer, T. Lahaye. Nucleic Acids Res. 2014, 42, 7436-7449.

\section{Computational details}

Conformational analyses were carried out whereby both BALLOON and confab programs ${ }^{1,2}$ in order to confirm the stereochemistry structure of $\mathbf{2 a}, \mathbf{3 a}, \mathbf{4}$, and $\mathbf{7}$. The BALLOON program explores conformational spaces with genetic algorithm, and synchronously, the confab program systematically generates diverse low energy conformations that are proposed to be close to crystal structures. The conformations generated by the above programs were assembled together via the removal of duplicated conformations whose root mean square (RMS) distance was less than $0.5 \AA$. Semi-empirical PM3 quantum mechanical geometry optimizations were fulfilled on conformations through the Gaussian 09 program. ${ }^{3}$ Duplicated conformations after geometry optimization were subsequently identified and disposed. Remaining conformations were further optimized at B3LYP/6-31G* level of theory in methanol solvent with IEFPCM3 solvation model using Gaussian 09 program, ${ }^{4}$ and duplicated conformations emerging after these calculations were removed according to the same RMS criteria above. Harmonic vibrational frequencies were performed to establish the stability of the finally obtained conformers. Oscillator strengths and rotational strengths of 20 weakest electronic excitations of each conformer were calculated by the TDDFT methodology at the B3LYP/6-311++G** level of theory adopting methanol as solvent by the IEFPCM solvation model carried out in Gaussian 09 program. The ECD spectra data for each conformer were then simulated by using a Gaussian function with a bandwidth $\sigma$ of $0.45 \mathrm{eV}$. Calculated spectra for each conformation were combined after Boltzmann weighting according to their population contribution.

1. M. J. Vainio, M. S. Johnson, 2007. Generating conformer ensembles using a multiobjective genetic algorithm. J. Chem. Inf. Model, 47, 2462-2474.

2. N. M.O'Boyle, T. Vandermeersch, C. J. Flynn, A. R. Maguire, G. R. Hutchison, 2011. Confab-systematic generation of diverse low-energy conformers. J. Cheminformatics, 3, 1-9.

3. M. J. Frisch, G. W. Trucks, H. B. Schlegel, G. E. Scuseria, M. A. Robb, J. R. Cheeseman, G. Scalmani, V. Barone, B. Mennucci, G. A. Petersson, H. Nakatsuji, M. Caricato, X. Li, H. P. Hratchian, A. F. Izmaylov, J. Bloino, G. Zheng, J. L. Sonnenberg, M. Hada, M. Ehara, K. Toyota, R. Fukuda, J. Hasegawa, M. Ishida, T. Nakajima, Y. Honda, O. Kitao, H. Nakai, T. Vreven, J. A. Montgomery, J. E. Peralta, F. Ogliaro, M. Bearpark, J. J. Heyd, E. Brothers, K. N. Kudin, V. N. Staroverov, T. Keith, R. Kobayashi, J. Normand, K. Raghavachari, A. Rendell, J. C. Burant, S. S. Iyengar, J. Tomasi, M. Cossi, N. Rega, J. M. Millam, M. Klene, J. E. Knox, J. B. Cross, V. Bakken, C. Adamo, J. Jaramillo, R. Gomperts, R. E. Stratmann, O. Yazyev, A. J. Austin, R. Cammi, C. Pomelli, J. W. Ochterski, R. L. Martin, K. Morokuma, V. G. Zakrzewski, G A. Voth, P. 
Salvador, J. J. Dannenberg, , S. Dapprich, A. D. Daniels, O. Farkas, J. B. Foresman, J. V. Ortiz, J. Cioslowski, D. J. Fox, Gaussian, Inc., Wallingford CT, 2010.

4. J. Tomasi, B. Mennucci, R. Cammi, 2005. Quantum mechanical continuum solvation models. Chem. Rev., 105, 2999-3094.

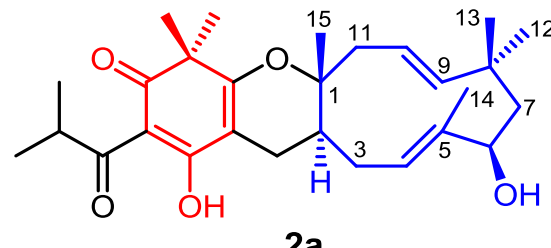

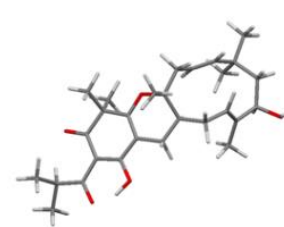

2a(1)

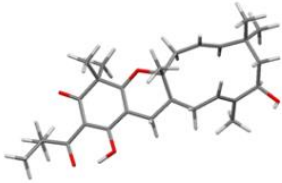

2a(4)

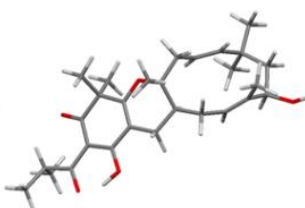

2a(2)

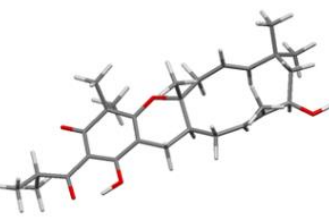

2a(3)

Optimized geometries of predominant conformers for compound 2a at the B3LYP/6-31G (d,p) level in methanol solution.

Important thermodynamic parameters (a.u.) and Boltzmann distributions of the optimized compound 2a at B3LYP/6-31G(d,p) level in methanol solution.

\begin{tabular}{llll}
\hline Conformation & E+ZPE & G & $\%$ \\
\hline $\mathbf{2 a}(1)$ & -1466.536450 & -1466.600733 & 3.00 \\
$\mathbf{2 a}(2)$ & -1466.540095 & -1466.603845 & 80.95 \\
$\mathbf{2 a}(3)$ & -1466.535366 & -1466.601963 & 11.04 \\
$\mathbf{2 a}(4)$ & -1466.534938 & -1466.599957 & 1.32 \\
$\mathbf{2 a}(5)$ & -1466.536472 & -1466.600930 & 3.70 \\
\hline
\end{tabular}

E+ZPE, G: total energy with zero point energy (ZPE) and Gibbs free energy in methanol solution at B3LYP/6-31G $(\mathrm{d}, \mathrm{p})$ level. \%: Boltzmann distributions, using the relative Gibbs free energies as weighting factors.

Optimized Z-matrixes of compound 2a in methanol solution $(\AA)$ at B3LYP/6-31G (d,p) level.

\begin{tabular}{|l|l|l|l|l|l|l|l|}
\hline \multicolumn{5}{|c|}{$\mathbf{2 a}(1)$} & \multicolumn{5}{c|}{ 2a (2) } \\
\hline C & -2.902271 & -1.634344 & -0.001736 & C & 2.753529 & 1.457572 & 0.379396 \\
\hline C & -4.222335 & -1.432731 & -0.009089 & C & 4.068039 & 1.305295 & 0.196871 \\
\hline C & -3.503137 & 1.278501 & 1.107317 & C & 3.174349 & -1.785831 & -0.181681 \\
\hline C & 3.795729 & 0.340095 & -0.136073 & C & -3.953844 & -0.319291 & -0.193053 \\
\hline C & 1.4302 & 0.376274 & 0.767492 & C & -1.547649 & -0.711264 & 0.48837 \\
\hline C & 2.696402 & 0.992546 & 0.493392 & C & -2.838208 & -1.167916 & 0.06026 \\
\hline C & -3.762864 & 1.913956 & -0.045718 & C & 4.505698 & -1.683632 & -0.065762 \\
\hline C & 1.25213 & -0.931436 & 0.431894 & C & -1.368823 & 0.618615 & 0.725113 \\
\hline
\end{tabular}




\begin{tabular}{|c|c|c|c|c|c|c|c|}
\hline $\mathrm{C}$ & 3.639768 & -1.057267 & -0.513005 & $\mathrm{C}$ & -3.763228 & 1.118194 & -0.063437 \\
\hline $\mathrm{C}$ & 5.003509 & 1.118393 & -0.384323 & $\mathrm{C}$ & -5.214432 & -0.940007 & -0.579479 \\
\hline $\mathrm{C}$ & -2.149919 & -1.910036 & 1.273984 & $\mathrm{C}$ & 2.092014 & 1.173275 & 1.703796 \\
\hline $\mathrm{C}$ & -2.190236 & 1.294974 & 1.860905 & $\mathrm{C}$ & 2.127342 & -1.93978 & 0.894958 \\
\hline $\mathrm{C}$ & 0.306045 & 1.183489 & 1.368049 & $\mathrm{C}$ & -0.407101 & -1.68653 & 0.641152 \\
\hline $\mathrm{C}$ & -5.820456 & 0.376918 & -0.671808 & $\mathrm{C}$ & 5.862269 & 0.20228 & -1.172753 \\
\hline $\mathrm{C}$ & -5.065493 & 1.715285 & -0.812351 & $\mathrm{C}$ & 5.298866 & -1.23881 & -1.2811 \\
\hline $\mathrm{C}$ & -1.050659 & 0.484488 & 1.163559 & $\mathrm{C}$ & 0.941484 & -0.947868 & 0.676033 \\
\hline $\mathrm{C}$ & -5.127813 & -0.956929 & -1.125536 & $\mathrm{C}$ & 4.880436 & 1.414955 & -1.079257 \\
\hline $\mathrm{C}$ & 2.291287 & -1.781201 & -0.255206 & $\mathrm{C}$ & -2.429962 & 1.668638 & 0.508156 \\
\hline $\mathrm{C}$ & -0.920614 & -1.012196 & 1.553909 & $\mathrm{C}$ & 0.857938 & 0.241728 & 1.666576 \\
\hline $\mathrm{C}$ & -2.818092 & 2.889465 & -0.707966 & $\mathrm{C}$ & 5.280945 & -1.908712 & 1.208222 \\
\hline $\mathrm{C}$ & -6.248642 & -2.010364 & -1.306891 & $\mathrm{C}$ & 5.754255 & 2.691733 & -0.986366 \\
\hline $\mathrm{C}$ & -4.387429 & -0.782475 & -2.461354 & $\mathrm{C}$ & 3.997793 & 1.510401 & -2.336081 \\
\hline $\mathrm{C}$ & 2.591432 & -3.025827 & 0.619845 & $\mathrm{C}$ & -2.769933 & 2.327113 & 1.873678 \\
\hline $\mathrm{C}$ & 1.739525 & -2.232389 & -1.634051 & $\mathrm{C}$ & -1.891727 & 2.742671 & -0.470292 \\
\hline $\mathrm{C}$ & -0.453693 & -1.239914 & 2.99733 & $\mathrm{C}$ & 0.48127 & -0.163913 & 3.096049 \\
\hline $\mathrm{C}$ & 7.099823 & -0.181278 & -0.006006 & $\mathrm{C}$ & -7.73884 & -0.963403 & -0.531774 \\
\hline $\mathrm{C}$ & 7.044107 & 1.636032 & -1.780659 & $\mathrm{C}$ & -6.474719 & 0.298751 & -2.338562 \\
\hline $\mathrm{C}$ & 6.238865 & 0.543305 & -1.065376 & $\mathrm{C}$ & -6.483063 & -0.143806 & -0.858192 \\
\hline $\mathrm{O}$ & 4.527442 & -1.739691 & -1.037596 & $\mathrm{O}$ & -4.615418 & 1.961463 & -0.36528 \\
\hline $\mathrm{O}$ & 5.064503 & 2.324573 & -0.017936 & $\mathrm{O}$ & -5.289481 & -2.19355 & -0.706158 \\
\hline $\mathrm{O}$ & 0.11921 & -1.601402 & 0.673911 & $\mathrm{O}$ & -0.220883 & 1.136403 & 1.178768 \\
\hline $\mathrm{O}$ & 2.786636 & 2.253515 & 0.868133 & $\mathrm{O}$ & -2.937289 & -2.47409 & -0.091862 \\
\hline $\mathrm{O}$ & -5.977119 & 2.803672 & -0.529981 & $\mathrm{O}$ & 6.396052 & -2.155841 & -1.44813 \\
\hline $\mathrm{H}$ & -2.314977 & -1.53538 & -0.913393 & $\mathrm{H}$ & 2.103213 & 1.739748 & -0.447241 \\
\hline $\mathrm{H}$ & -4.749832 & -1.556199 & 0.940784 & $\mathrm{H}$ & 4.664289 & 1.033811 & 1.071103 \\
\hline $\mathrm{H}$ & -4.266532 & 0.623427 & 1.513347 & $\mathrm{H}$ & 2.753996 & -1.648831 & -1.179638 \\
\hline $\mathrm{H}$ & -2.83736 & -1.820324 & 2.123124 & $\mathrm{H}$ & 2.829564 & 0.735221 & 2.385792 \\
\hline $\mathrm{H}$ & -1.765485 & -2.939753 & 1.29575 & $\mathrm{H}$ & 1.742376 & 2.100409 & 2.180666 \\
\hline $\mathrm{H}$ & -1.830503 & 2.326079 & 1.96402 & $\mathrm{H}$ & 1.718393 & -2.960274 & 0.869901 \\
\hline $\mathrm{H}$ & -2.34409 & 0.933668 & 2.883582 & $\mathrm{H}$ & 2.563029 & -1.820107 & 1.891652 \\
\hline $\mathrm{H}$ & 0.486327 & 1.379486 & 2.434305 & $\mathrm{H}$ & -0.533425 & -2.30655 & 1.539653 \\
\hline $\mathrm{H}$ & 0.272874 & 2.168062 & 0.889075 & $\mathrm{H}$ & -0.408392 & -2.384771 & -0.203139 \\
\hline $\mathrm{H}$ & -6.168942 & 0.257324 & 0.363944 & $\mathrm{H}$ & 6.545277 & 0.234972 & -0.31415 \\
\hline $\mathrm{H}$ & -6.727368 & 0.50309 & -1.276825 & $\mathrm{H}$ & 6.488036 & 0.363359 & -2.063552 \\
\hline $\mathrm{H}$ & -4.839037 & 1.853953 & -1.8757 & $\mathrm{H}$ & 4.642423 & -1.293995 & -2.161464 \\
\hline $\mathrm{H}$ & -1.258542 & 0.495248 & 0.087792 & $\mathrm{H}$ & 1.072693 & -0.48843 & -0.311005 \\
\hline $\mathrm{H}$ & -1.997846 & 3.203682 & -0.056917 & $\mathrm{H}$ & 5.862198 & -1.024124 & 1.497828 \\
\hline $\mathrm{H}$ & -3.367984 & 3.787324 & -1.016906 & $\mathrm{H}$ & 6.002677 & -2.722059 & 1.069402 \\
\hline $\mathrm{H}$ & -2.375465 & 2.462086 & -1.61907 & $\mathrm{H}$ & 4.632624 & -2.174214 & 2.046842 \\
\hline $\mathrm{H}$ & -6.947978 & -1.702814 & -2.093748 & $\mathrm{H}$ & 6.407661 & 2.782406 & -1.86308 \\
\hline
\end{tabular}




\begin{tabular}{|c|c|c|c|c|c|c|c|}
\hline $\mathrm{H}$ & -6.820845 & -2.143912 & -0.380783 & $\mathrm{H}$ & 6.389399 & 2.675031 & -0.092668 \\
\hline $\mathrm{H}$ & -5.82553 & -2.982478 & -1.585139 & $\mathrm{H}$ & 5.123152 & 3.586106 & -0.935384 \\
\hline $\mathrm{H}$ & -3.991627 & -1.742736 & -2.811749 & $\mathrm{H}$ & 3.383342 & 2.417701 & -2.315441 \\
\hline $\mathrm{H}$ & -3.545774 & -0.086913 & -2.378739 & $\mathrm{H}$ & 3.323079 & 0.653455 & -2.429757 \\
\hline $\mathrm{H}$ & -5.067494 & -0.400787 & -3.23192 & $\mathrm{H}$ & 4.620551 & 1.553505 & -3.237387 \\
\hline $\mathrm{H}$ & 3.358584 & -3.627707 & 0.12801 & $\mathrm{H}$ & -3.544185 & 3.083384 & 1.721453 \\
\hline $\mathrm{H}$ & 1.687453 & -3.625348 & 0.750632 & $\mathrm{H}$ & -1.88028 & 2.801347 & 2.296058 \\
\hline $\mathrm{H}$ & 2.960176 & -2.732914 & 1.609213 & $\mathrm{H}$ & -3.142396 & 1.585773 & 2.589273 \\
\hline $\mathrm{H}$ & 0.833501 & -2.829594 & -1.502291 & $\mathrm{H}$ & -1.012112 & 3.234682 & -0.048441 \\
\hline $\mathrm{H}$ & 2.498746 & -2.834536 & -2.138857 & $\mathrm{H}$ & -2.672579 & 3.485165 & -0.64684 \\
\hline $\mathrm{H}$ & 1.500072 & -1.370181 & -2.266061 & $\mathrm{H}$ & -1.613178 & 2.296035 & -1.431206 \\
\hline $\mathrm{H}$ & -0.400739 & -2.312757 & 3.207205 & $\mathrm{H}$ & 0.459925 & 0.718452 & 3.743322 \\
\hline $\mathrm{H}$ & -1.157588 & -0.79049 & 3.704391 & $\mathrm{H}$ & 1.2164 & -0.866156 & 3.501319 \\
\hline $\mathrm{H}$ & 0.534551 & -0.809223 & 3.178299 & $\mathrm{H}$ & -0.502554 & -0.638889 & 3.136179 \\
\hline $\mathrm{H}$ & 6.549739 & -1.003939 & 0.45862 & $\mathrm{H}$ & -7.749234 & -1.28204 & 0.516599 \\
\hline $\mathrm{H}$ & 7.996789 & -0.596297 & -0.479273 & $\mathrm{H}$ & -8.631156 & -0.352693 & -0.708881 \\
\hline $\mathrm{H}$ & 7.420677 & 0.516391 & 0.776666 & $\mathrm{H}$ & -7.805696 & -1.859286 & -1.155843 \\
\hline $\mathrm{H}$ & 7.897438 & 1.182407 & -2.297112 & $\mathrm{H}$ & -7.386111 & 0.865092 & -2.560953 \\
\hline $\mathrm{H}$ & 7.425661 & 2.380898 & -1.076136 & $\mathrm{H}$ & -6.444526 & -0.57227 & -3.003828 \\
\hline $\mathrm{H}$ & 6.435831 & 2.159779 & -2.526378 & $\mathrm{H}$ & -5.615219 & 0.93869 & -2.555197 \\
\hline $\mathrm{H}$ & 5.910731 & -0.207631 & -1.785848 & $\mathrm{H}$ & -6.461112 & 0.760443 & -0.247887 \\
\hline $\mathrm{H}$ & 3.736074 & 2.550929 & 0.594688 & $\mathrm{H}$ & -3.912367 & -2.640197 & -0.383919 \\
\hline $\mathrm{H}$ & -6.100087 & 2.810748 & 0.433984 & $\mathrm{H}$ & 6.938196 & -1.82773 & -2.183891 \\
\hline \multicolumn{4}{|c|}{ 2a (3) } & \multicolumn{4}{|c|}{$2 \mathbf{a}(4)$} \\
\hline $\mathrm{C}$ & 3.373891 & -1.67322 & -0.240272 & $\mathrm{C}$ & 3.373891 & -1.67322 & -0.240272 \\
\hline $\mathrm{C}$ & 4.321481 & -1.21635 & 0.582155 & $\mathrm{C}$ & 4.321481 & -1.21635 & 0.582155 \\
\hline $\mathrm{C}$ & 3.390007 & 1.39864 & -0.670321 & $\mathrm{C}$ & 3.390007 & 1.39864 & -0.670321 \\
\hline $\mathrm{C}$ & -4.167384 & 0.301476 & -0.009438 & $\mathrm{C}$ & -4.167384 & 0.301476 & -0.009438 \\
\hline $\mathrm{C}$ & -1.68284 & 0.459077 & -0.451891 & $\mathrm{C}$ & -1.68284 & 0.459077 & -0.451891 \\
\hline $\mathrm{C}$ & -2.993591 & 1.028491 & -0.366358 & $\mathrm{C}$ & -2.993591 & 1.028491 & -0.366358 \\
\hline $\mathrm{C}$ & 4.072818 & 1.905791 & 0.367231 & $\mathrm{C}$ & 4.072818 & 1.905791 & 0.367231 \\
\hline $\mathrm{C}$ & -1.525041 & -0.869066 & -0.213348 & $\mathrm{C}$ & -1.525041 & -0.869066 & -0.213348 \\
\hline $\mathrm{C}$ & -4.023907 & -1.105354 & 0.3352 & $\mathrm{C}$ & -4.023907 & -1.105354 & 0.3352 \\
\hline $\mathrm{C}$ & -5.439713 & 1.010599 & -0.010272 & $\mathrm{C}$ & -5.439713 & 1.010599 & -0.010272 \\
\hline $\mathrm{C}$ & 1.912916 & -1.681095 & 0.116836 & $\mathrm{C}$ & 1.912916 & -1.681095 & 0.116836 \\
\hline $\mathrm{C}$ & 1.942734 & 1.617927 & -1.020414 & $\mathrm{C}$ & 1.942734 & 1.617927 & -1.020414 \\
\hline $\mathrm{C}$ & -0.497295 & 1.304737 & -0.817045 & $\mathrm{C}$ & -0.497295 & 1.304737 & -0.817045 \\
\hline $\mathrm{C}$ & 6.149568 & 0.381542 & -0.156799 & $\mathrm{C}$ & 6.149568 & 0.381542 & -0.156799 \\
\hline $\mathrm{C}$ & 5.544486 & 1.574715 & 0.606759 & $\mathrm{C}$ & 5.544486 & 1.574715 & 0.606759 \\
\hline $\mathrm{C}$ & 0.856192 & 0.68638 & -0.391743 & $\mathrm{C}$ & 0.856192 & 0.68638 & -0.391743 \\
\hline $\mathrm{C}$ & 5.810191 & -1.073822 & 0.321046 & $\mathrm{C}$ & 5.810191 & -1.073822 & 0.321046 \\
\hline $\mathrm{C}$ & -2.64567 & -1.801802 & 0.176353 & $\mathrm{C}$ & -2.64567 & -1.801802 & 0.176353 \\
\hline
\end{tabular}




\begin{tabular}{|c|c|c|c|c|c|c|c|}
\hline $\mathrm{C}$ & 0.918895 & -0.833385 & -0.734624 & $\mathrm{C}$ & 0.918895 & -0.833385 & -0.734624 \\
\hline $\mathrm{C}$ & 3.488522 & 2.839917 & 1.39972 & $\mathrm{C}$ & 3.488522 & 2.839917 & 1.39972 \\
\hline $\mathrm{C}$ & 6.307241 & -2.046907 & -0.764825 & $\mathrm{C}$ & 6.307241 & -2.046907 & -0.764825 \\
\hline $\mathrm{C}$ & 6.563795 & -1.376253 & 1.636398 & $\mathrm{C}$ & 6.563795 & -1.376253 & 1.636398 \\
\hline $\mathrm{C}$ & -2.811265 & -2.87629 & -0.932494 & $\mathrm{C}$ & -2.811265 & -2.87629 & -0.932494 \\
\hline $\mathrm{C}$ & -2.297849 & -2.487748 & 1.521342 & $\mathrm{C}$ & -2.297849 & -2.487748 & 1.521342 \\
\hline $\mathrm{C}$ & 1.040634 & -1.120009 & -2.231142 & $\mathrm{C}$ & 1.040634 & -1.120009 & -2.231142 \\
\hline $\mathrm{C}$ & -7.935688 & 1.020485 & -0.396451 & $\mathrm{C}$ & -7.935688 & 1.020485 & -0.396451 \\
\hline $\mathrm{C}$ & -6.962643 & 0.374693 & 1.860263 & $\mathrm{C}$ & -6.962643 & 0.374693 & 1.860263 \\
\hline $\mathrm{C}$ & -6.765955 & 0.340868 & 0.32813 & $\mathrm{C}$ & -6.765955 & 0.340868 & 0.32813 \\
\hline $\mathrm{O}$ & -4.947207 & -1.818339 & 0.74533 & $\mathrm{O}$ & -4.947207 & -1.818339 & 0.74533 \\
\hline $\mathrm{O}$ & -5.478649 & 2.24102 & -0.290968 & $\mathrm{O}$ & -5.478649 & 2.24102 & -0.290968 \\
\hline $\mathrm{O}$ & -0.351179 & -1.490813 & -0.298695 & $\mathrm{O}$ & -0.351179 & -1.490813 & -0.298695 \\
\hline $\mathrm{O}$ & -3.060099 & 2.314656 & -0.651676 & $\mathrm{O}$ & -3.060099 & 2.314656 & -0.651676 \\
\hline $\mathrm{O}$ & 6.341976 & 2.757536 & 0.359404 & $\mathrm{O}$ & 6.341976 & 2.757536 & 0.359404 \\
\hline $\mathrm{H}$ & 3.637062 & -2.066569 & -1.221174 & $\mathrm{H}$ & 3.637062 & -2.066569 & -1.221174 \\
\hline $\mathrm{H}$ & 3.996375 & -0.822629 & 1.547454 & $\mathrm{H}$ & 3.996375 & -0.822629 & 1.547454 \\
\hline $\mathrm{H}$ & 3.91284 & 0.72726 & -1.34131 & $\mathrm{H}$ & 3.91284 & 0.72726 & -1.34131 \\
\hline $\mathrm{H}$ & 1.537766 & -2.709825 & 0.030898 & $\mathrm{H}$ & 1.537766 & -2.709825 & 0.030898 \\
\hline $\mathrm{H}$ & 1.784269 & -1.388292 & 1.165738 & $\mathrm{H}$ & 1.784269 & -1.388292 & 1.165738 \\
\hline $\mathrm{H}$ & 1.642522 & 2.633614 & -0.737348 & $\mathrm{H}$ & 1.642522 & 2.633614 & -0.737348 \\
\hline $\mathrm{H}$ & 1.836767 & 1.571598 & -2.111457 & $\mathrm{H}$ & 1.836767 & 1.571598 & -2.111457 \\
\hline $\mathrm{H}$ & -0.490928 & 1.498347 & -1.899448 & $\mathrm{H}$ & -0.490928 & 1.498347 & -1.899448 \\
\hline $\mathrm{H}$ & -0.590021 & 2.287958 & -0.344894 & $\mathrm{H}$ & -0.590021 & 2.287958 & -0.344894 \\
\hline $\mathrm{H}$ & 5.907254 & 0.472782 & -1.223056 & $\mathrm{H}$ & 5.907254 & 0.472782 & -1.223056 \\
\hline $\mathrm{H}$ & 7.237614 & 0.502094 & -0.084738 & $\mathrm{H}$ & 7.237614 & 0.502094 & -0.084738 \\
\hline $\mathrm{H}$ & 5.678605 & 1.406172 & 1.68304 & $\mathrm{H}$ & 5.678605 & 1.406172 & 1.68304 \\
\hline $\mathrm{H}$ & 0.933082 & 0.761983 & 0.700603 & $\mathrm{H}$ & 0.933082 & 0.761983 & 0.700603 \\
\hline $\mathrm{H}$ & 2.500506 & 3.218199 & 1.124802 & $\mathrm{H}$ & 2.500506 & 3.218199 & 1.124802 \\
\hline $\mathrm{H}$ & 4.154901 & 3.696472 & 1.554472 & $\mathrm{H}$ & 4.154901 & 3.696472 & 1.554472 \\
\hline $\mathrm{H}$ & 3.39158 & 2.33361 & 2.370876 & $\mathrm{H}$ & 3.39158 & 2.33361 & 2.370876 \\
\hline $\mathrm{H}$ & 7.385256 & -1.923083 & -0.921464 & $\mathrm{H}$ & 7.385256 & -1.923083 & -0.921464 \\
\hline $\mathrm{H}$ & 5.813029 & -1.8713 & -1.726667 & $\mathrm{H}$ & 5.813029 & -1.8713 & -1.726667 \\
\hline $\mathrm{H}$ & 6.123274 & -3.087144 & -0.473102 & $\mathrm{H}$ & 6.123274 & -3.087144 & -0.473102 \\
\hline $\mathrm{H}$ & 6.359292 & -2.399766 & 1.972086 & $\mathrm{H}$ & 6.359292 & -2.399766 & 1.972086 \\
\hline $\mathrm{H}$ & 6.263068 & -0.698189 & 2.443555 & $\mathrm{H}$ & 6.263068 & -0.698189 & 2.443555 \\
\hline $\mathrm{H}$ & 7.646495 & -1.274989 & 1.49661 & $\mathrm{H}$ & 7.646495 & -1.274989 & 1.49661 \\
\hline $\mathrm{H}$ & -1.888876 & -3.452706 & -1.040534 & $\mathrm{H}$ & -1.888876 & -3.452706 & -1.040534 \\
\hline $\mathrm{H}$ & -3.049477 & -2.416427 & -1.897935 & $\mathrm{H}$ & -3.049477 & -2.416427 & -1.897935 \\
\hline $\mathrm{H}$ & -3.627657 & -3.548172 & -0.656252 & $\mathrm{H}$ & -3.627657 & -3.548172 & -0.656252 \\
\hline $\mathrm{H}$ & -2.143341 & -1.746787 & 2.313531 & $\mathrm{H}$ & -2.143341 & -1.746787 & 2.313531 \\
\hline $\mathrm{H}$ & -1.386859 & -3.082603 & 1.421134 & $\mathrm{H}$ & -1.386859 & -3.082603 & 1.421134 \\
\hline
\end{tabular}




\begin{tabular}{|c|c|c|c|c|c|c|c|}
\hline $\mathrm{H}$ & -3.125344 & -3.138299 & 1.81164 & $\mathrm{H}$ & -3.125344 & -3.138299 & 1.81164 \\
\hline $\mathrm{H}$ & 0.248944 & -0.620356 & -2.798257 & $\mathrm{H}$ & 0.248944 & -0.620356 & -2.798257 \\
\hline $\mathrm{H}$ & 0.966739 & -2.196355 & -2.414885 & $\mathrm{H}$ & 0.966739 & -2.196355 & -2.414885 \\
\hline $\mathrm{H}$ & 2.004952 & -0.77445 & -2.614697 & $\mathrm{H}$ & 2.004952 & -0.77445 & -2.614697 \\
\hline $\mathrm{H}$ & -7.800184 & 1.00094 & -1.48362 & $\mathrm{H}$ & -7.800184 & 1.00094 & -1.48362 \\
\hline $\mathrm{H}$ & -8.867437 & 0.493867 & -0.161543 & $\mathrm{H}$ & -8.867437 & 0.493867 & -0.161543 \\
\hline $\mathrm{H}$ & -8.044732 & 2.06509 & -0.090604 & $\mathrm{H}$ & -8.044732 & 2.06509 & -0.090604 \\
\hline $\mathrm{H}$ & -6.164904 & -0.168366 & 2.374059 & $\mathrm{H}$ & -6.164904 & -0.168366 & 2.374059 \\
\hline $\mathrm{H}$ & -7.917595 & -0.095139 & 2.121416 & $\mathrm{H}$ & -7.917595 & -0.095139 & 2.121416 \\
\hline $\mathrm{H}$ & -6.980653 & 1.408028 & 2.226472 & $\mathrm{H}$ & -6.980653 & 1.408028 & 2.226472 \\
\hline $\mathrm{H}$ & -6.70442 & -0.707249 & 0.031319 & $\mathrm{H}$ & -6.70442 & -0.707249 & 0.031319 \\
\hline $\mathrm{H}$ & -4.057127 & 2.56241 & -0.554576 & $\mathrm{H}$ & -4.057127 & 2.56241 & -0.554576 \\
\hline $\mathrm{H}$ & 6.171467 & 3.011554 & -0.563276 & $\mathrm{H}$ & 6.171467 & 3.011554 & -0.563276 \\
\hline \multicolumn{4}{|c|}{$\mathbf{2 a}(5)$} & & & & \\
\hline $\mathrm{C}$ & -2.908304 & -1.620772 & 0.101842 & & & & \\
\hline $\mathrm{C}$ & -4.226779 & -1.410904 & 0.07488 & & & & \\
\hline $\mathrm{C}$ & -3.49401 & 1.35798 & 1.028163 & & & & \\
\hline $\mathrm{C}$ & 3.806902 & 0.29783 & -0.091717 & & & & \\
\hline $\mathrm{C}$ & 1.432685 & 0.40599 & 0.778615 & & & & \\
\hline $\mathrm{C}$ & 2.712454 & 0.991186 & 0.500156 & & & & \\
\hline $\mathrm{C}$ & -3.734632 & 1.921986 & -0.165463 & & & & \\
\hline $\mathrm{C}$ & 1.245407 & -0.915918 & 0.509466 & & & & \\
\hline $\mathrm{C}$ & 3.599691 & -1.082808 & -0.504392 & & & & \\
\hline $\mathrm{C}$ & 5.063327 & 1.016131 & -0.261508 & & & & \\
\hline $\mathrm{C}$ & -2.164395 & -1.824719 & 1.395915 & & & & \\
\hline $\mathrm{C}$ & -2.188455 & 1.408049 & 1.793284 & & & & \\
\hline $\mathrm{C}$ & 0.311661 & 1.252694 & 1.328191 & & & & \\
\hline $\mathrm{C}$ & -5.801823 & 0.369805 & -0.714494 & & & & \\
\hline $\mathrm{C}$ & -5.02698 & 1.684731 & -0.938287 & & & & \\
\hline $\mathrm{C}$ & -1.048467 & 0.552241 & 1.152953 & & & & \\
\hline $\mathrm{C}$ & -5.123484 & -0.999938 & -1.074087 & & & & \\
\hline $\mathrm{C}$ & 2.283411 & -1.809173 & -0.122253 & & & & \\
\hline $\mathrm{C}$ & -0.929561 & -0.920959 & 1.628259 & & & & \\
\hline $\mathrm{C}$ & -2.771193 & 2.84451 & -0.875443 & & & & \\
\hline $\mathrm{C}$ & -6.25665 & -2.049348 & -1.188626 & & & & \\
\hline $\mathrm{C}$ & -4.377005 & -0.924214 & -2.415654 & & & & \\
\hline $\mathrm{C}$ & 2.670139 & -2.921089 & 0.891767 & & & & \\
\hline $\mathrm{C}$ & 1.694687 & -2.451461 & -1.403259 & & & & \\
\hline $\mathrm{C}$ & -0.468148 & -1.069893 & 3.083469 & & & & \\
\hline $\mathrm{C}$ & 7.58774 & 0.997995 & -0.251321 & & & & \\
\hline $\mathrm{C}$ & 6.283248 & 0.5288 & -2.381145 & & & & \\
\hline $\mathrm{C}$ & 6.316456 & 0.374834 & -0.844248 & & & & \\
\hline
\end{tabular}




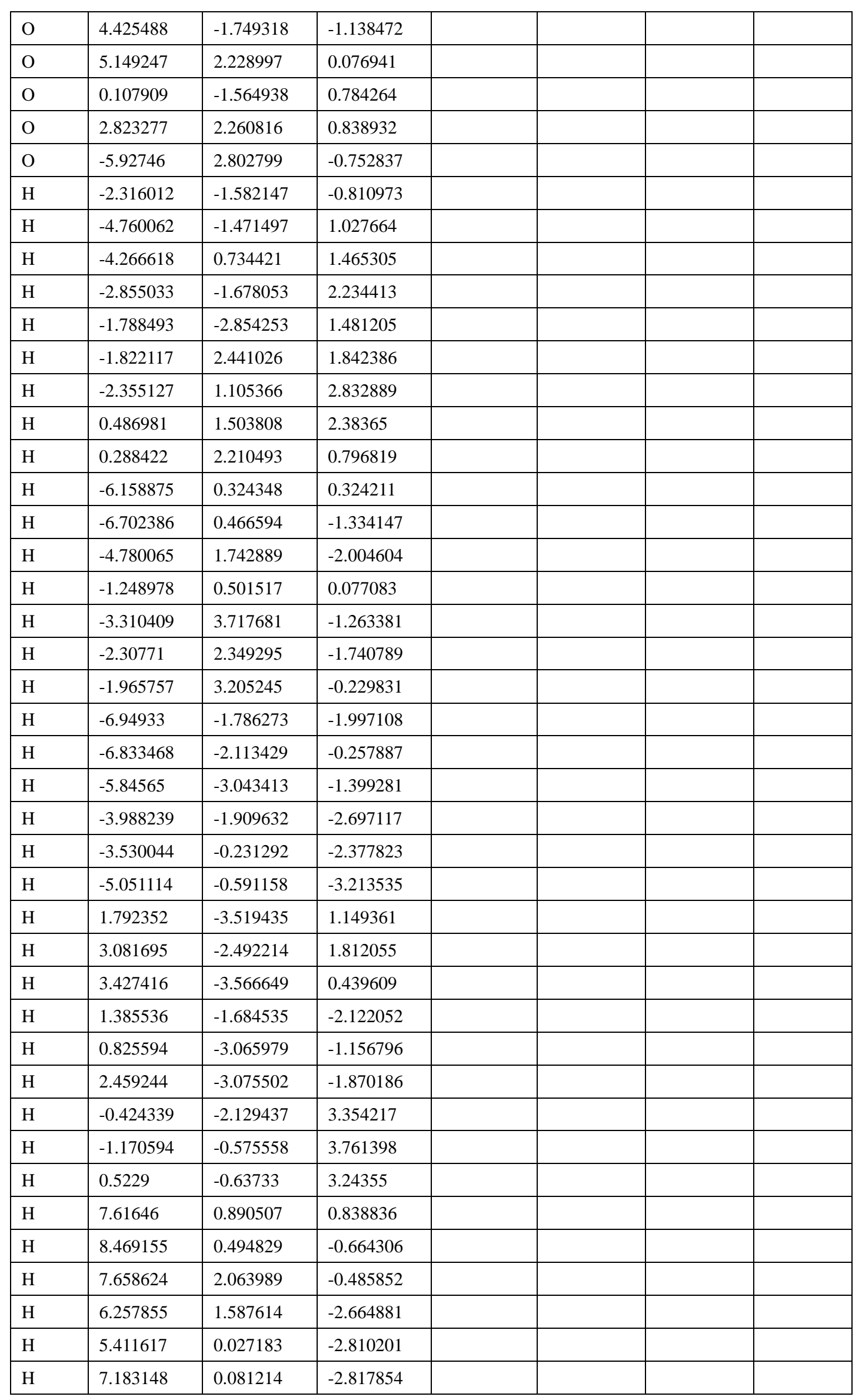




\begin{tabular}{|l|l|l|l|l|l|l|l|}
\hline $\mathrm{H}$ & 6.289955 & -0.693219 & -0.623239 & & & & \\
\hline $\mathrm{H}$ & 3.789001 & 2.524814 & 0.590995 & & & & \\
\hline $\mathrm{H}$ & -6.070757 & 2.87993 & 0.205191 & & & & \\
\hline
\end{tabular}

Key transitions, oscillator strengths, and rotatory strengths in the ECD of conformers 2a (2) at B3LYP /6-311++G (d,p) level.

\begin{tabular}{|c|c|c|c|c|c|}
\hline Species & Excited State & $\Delta \mathrm{E}(\mathrm{eV})^{\mathrm{a}}$ & $\lambda(\mathrm{nm})^{\mathrm{b}}$ & $\mathrm{f}^{\mathrm{c}}$ & Rvel $^{\mathrm{d}}$ \\
\hline \multirow[t]{35}{*}{$\mathbf{2 a}(2)$} & $124->125$ & 3.7143 & 333.81 & 0.1821 & 3.7502 \\
\hline & $123->125$ & 3.7911 & 327.04 & 0.2465 & 7.87 \\
\hline & $122->125$ & 3.947 & 314.13 & 0.0009 & -10.5191 \\
\hline & $120->125$ & 4.1948 & 295.57 & 0.0012 & 3.739 \\
\hline & $122->126$ & 4.3641 & 284.1 & 0.0069 & 4.8168 \\
\hline & $123->126$ & 4.5296 & 273.72 & 0.1144 & -2.0356 \\
\hline & $124->126$ & 4.6411 & 267.15 & 0.0127 & 1.2825 \\
\hline & $121->125$ & 4.67 & 265.49 & 0.0012 & 2.7124 \\
\hline & $120->126$ & 5.0298 & 246.5 & 0.0008 & -5.8841 \\
\hline & $118->125$ & 5.2204 & 237.5 & 0.0652 & 18.2841 \\
\hline & $119->125$ & 5.312 & 233.4 & 0.0001 & 0.0311 \\
\hline & $124->127$ & 5.3269 & 232.75 & 0.0119 & 3.3372 \\
\hline & $124->128$ & 5.4047 & 229.4 & 0.0074 & 71.3781 \\
\hline & $123->127$ & 5.4199 & 228.76 & 0.0021 & 8.2966 \\
\hline & $121->126$ & 5.5662 & 222.75 & 0.0045 & -0.402 \\
\hline & $124->130$ & 5.5914 & 221.74 & 0.004 & -3.0217 \\
\hline & $124->129$ & 5.6229 & 220.5 & 0.0043 & -2.4207 \\
\hline & $123->128$ & 5.6746 & 218.49 & 0.0106 & 1.6857 \\
\hline & $118->126$ & 5.7118 & 217.07 & 0.1508 & 52.1581 \\
\hline & $124->131$ & 5.7594 & 215.27 & 0.0099 & -8.2621 \\
\hline & $123->129$ & 5.7861 & 214.28 & 0.0129 & -11.4785 \\
\hline & $116->125$ & 5.79 & 214.13 & 0.0228 & -26.5884 \\
\hline & $123->130$ & 5.8463 & 212.07 & 0.0052 & 2.9516 \\
\hline & $124->133$ & 5.8898 & 210.51 & 0.033 & 10.0918 \\
\hline & $123->131$ & 5.9182 & 209.5 & 0.0021 & 4.5115 \\
\hline & $124->133$ & 5.9599 & 208.03 & 0.015 & -4.1709 \\
\hline & $117->125$ & 5.9956 & 206.79 & 0.0155 & -5.4358 \\
\hline & $123->132$ & 6.0038 & 206.51 & 0.0015 & -0.8213 \\
\hline & $124->134$ & 6.0567 & 204.71 & 0.005 & -6.3543 \\
\hline & $122->127$ & 6.0693 & 204.28 & 0.0046 & 1.8185 \\
\hline & $124->135$ & 6.1213 & 202.55 & 0.0014 & -1.719 \\
\hline & $113->125$ & 6.1362 & 202.05 & 0.0038 & -0.6516 \\
\hline & $119->126$ & 6.151 & 201.57 & 0 & -0.0056 \\
\hline & $123->133$ & 6.1592 & 201.3 & 0.04 & -1.4409 \\
\hline & $123->134$ & 6.2027 & 199.89 & 0.0112 & 5.7707 \\
\hline
\end{tabular}




\begin{tabular}{|l|l|l|l|l|l|}
\hline & $124->139$ & 6.2232 & 199.23 & 0.0089 & 2.1238 \\
\hline & $124->136$ & 6.2452 & 198.53 & 0.0084 & 0.9906 \\
\hline & $124->137$ & 6.28 & 197.43 & 0.0166 & -10.8155 \\
\hline & $116->125$ & 6.2885 & 197.16 & 0.0003 & -0.5832 \\
\hline & $124->137$ & 6.3027 & 196.72 & 0.0101 & -2.0605 \\
\hline
\end{tabular}

${ }^{a}$ Excitation energy. ${ }^{b}$ Wavelength. ${ }^{c}$ Oscillator strength. ${ }^{\mathrm{d}}$ Rotatory strength in velocity form $(10-40 \mathrm{cgs}$.$) .$

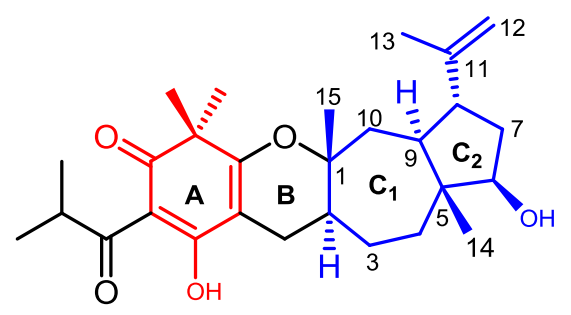

$3 \mathbf{a}$

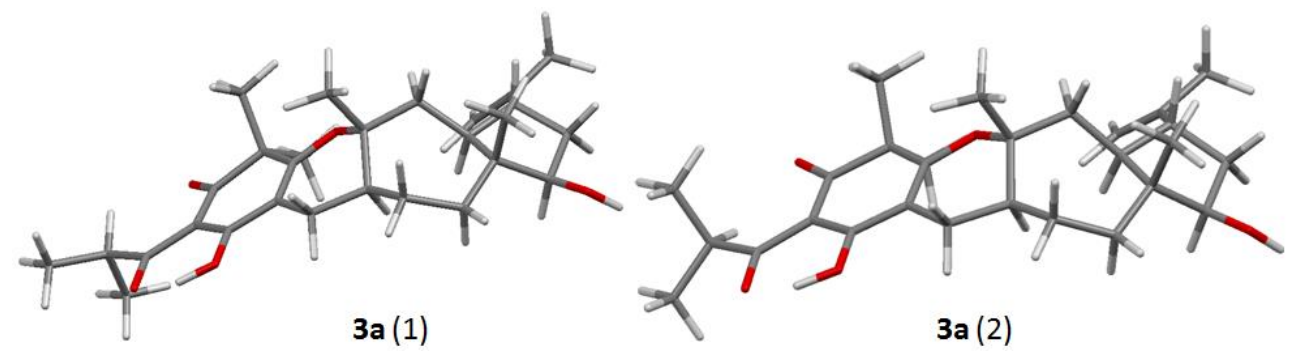

Optimized geometries of predominant conformers for compound 3a at the B3LYP/6-31G (d,p) level in methanol solution.

Important thermodynamic parameters (a.u.) and Boltzmann distributions of the optimized compound 3a at B3LYP/6-31G(d,p) levelin methanol solution

\begin{tabular}{cccc}
\hline Conformation & E+ZPE & G & $\%$ \\
\hline 3a $(1)$ & -1466.560344 & -1466.623502 & 43.79 \\
3a $(2)$ & -1466.560290 & -1466.623738 & 56.21
\end{tabular}

E+ZPE, G: total energy with zero point energy (ZPE) and Gibbs free energy in methanol solution at B3LYP/6-31G $(\mathrm{d}, \mathrm{p})$ level. \%: Boltzmann distributions, using the relative Gibbs free energies as weighting factors.

Optimized Z-matrixes of compound 3a in methanol solution $(\AA)$ at B3LYP/6-31G (d,p) level.

\begin{tabular}{|l|r|r|r|l|r|r|r|}
\hline \multicolumn{5}{|c|}{$3 \mathbf{a}(1)$} & \multicolumn{5}{|c|}{ 3a $(2)$} \\
\hline C & -3.881509 & -0.206812 & -0.128993 & C & -3.88151 & -0.20681 & -0.12899 \\
\hline C & -1.500539 & -0.991413 & 0.225158 & C & -1.50054 & -0.99141 & 0.225158 \\
\hline C & -2.884537 & -1.222619 & -0.07248 & C & -2.88454 & -1.22262 & -0.07248 \\
\hline C & -1.100836 & 0.275183 & 0.536132 & C & -1.10084 & 0.275183 & 0.536132 \\
\hline C & -3.463561 & 1.173339 & 0.071235 & C & -3.46356 & 1.173339 & 0.071235 \\
\hline C & 2.884872 & 2.958427 & -0.907598 & C & 2.884872 & 2.958427 & -0.9076 \\
\hline C & -5.255758 & -0.607499 & -0.401072 & C & -5.25576 & -0.6075 & -0.40107 \\
\hline C & 4.071304 & 2.620575 & -0.387536 & C & 4.071304 & 2.620575 & -0.38754 \\
\hline C & -0.508488 & -2.128758 & 0.157612 & C & -0.50849 & -2.12876 & 0.157612 \\
\hline C & 1.990901 & -2.6946 & 0.099818 & C & 1.990901 & -2.6946 & 0.099818 \\
\hline
\end{tabular}




\begin{tabular}{|c|c|c|c|c|c|c|c|}
\hline $\mathrm{C}$ & 3.225886 & -2.361281 & -0.757459 & $\mathrm{C}$ & 3.225886 & -2.36128 & -0.75746 \\
\hline $\mathrm{C}$ & 5.614098 & 0.627079 & -0.925761 & $\mathrm{C}$ & 5.614098 & 0.627079 & -0.92576 \\
\hline $\mathrm{C}$ & 2.488637 & 0.183515 & 1.239772 & $\mathrm{C}$ & 2.488637 & 0.183515 & 1.239772 \\
\hline $\mathrm{C}$ & 4.481364 & 1.205913 & -0.011944 & $\mathrm{C}$ & 4.481364 & 1.205913 & -0.01194 \\
\hline $\mathrm{C}$ & 0.924879 & -1.578307 & 0.108704 & $\mathrm{C}$ & 0.924879 & -1.57831 & 0.108704 \\
\hline $\mathrm{C}$ & 3.369999 & 0.119458 & -0.016014 & $\mathrm{C}$ & 3.369999 & 0.119458 & -0.01601 \\
\hline $\mathrm{C}$ & 5.120217 & -0.770432 & -1.356378 & $\mathrm{C}$ & 5.120217 & -0.77043 & -1.35638 \\
\hline $\mathrm{C}$ & -2.014894 & 1.475696 & 0.535323 & $\mathrm{C}$ & -2.01489 & 1.475696 & 0.535323 \\
\hline $\mathrm{C}$ & 4.134601 & -1.221925 & -0.250945 & $\mathrm{C}$ & 4.134601 & -1.22193 & -0.25095 \\
\hline $\mathrm{C}$ & 1.106417 & -0.509444 & 1.208543 & $\mathrm{C}$ & 1.106417 & -0.50944 & 1.208543 \\
\hline $\mathrm{C}$ & 5.128338 & 3.669731 & -0.124472 & $\mathrm{C}$ & 5.128338 & 3.669731 & -0.12447 \\
\hline $\mathrm{C}$ & -1.42621 & 2.571448 & -0.38722 & $\mathrm{C}$ & -1.42621 & 2.571448 & -0.38722 \\
\hline $\mathrm{C}$ & -2.123559 & 2.018136 & 1.987976 & $\mathrm{C}$ & -2.12356 & 2.018136 & 1.987976 \\
\hline $\mathrm{C}$ & 4.9184 & -1.694673 & 0.99163 & $\mathrm{C}$ & 4.9184 & -1.69467 & 0.99163 \\
\hline $\mathrm{C}$ & 0.747933 & -0.988558 & 2.621681 & $\mathrm{C}$ & 0.747933 & -0.98856 & 2.621681 \\
\hline $\mathrm{C}$ & -7.731308 & -0.270852 & -0.032586 & $\mathrm{C}$ & -7.73131 & -0.27085 & -0.03259 \\
\hline $\mathrm{C}$ & -6.509972 & 0.928825 & -1.910577 & $\mathrm{C}$ & -6.50997 & 0.928825 & -1.91058 \\
\hline $\mathrm{C}$ & -6.412495 & 0.381551 & -0.469037 & $\mathrm{C}$ & -6.4125 & 0.381551 & -0.46904 \\
\hline $\mathrm{O}$ & -4.204158 & 2.151646 & -0.079436 & $\mathrm{O}$ & -4.20416 & 2.151646 & -0.07944 \\
\hline $\mathrm{O}$ & -5.532731 & -1.82248 & -0.601625 & $\mathrm{O}$ & -5.53273 & -1.82248 & -0.60163 \\
\hline $\mathrm{O}$ & 0.153355 & 0.586177 & 0.887856 & $\mathrm{O}$ & 0.153355 & 0.586177 & 0.887856 \\
\hline $\mathrm{O}$ & -3.194824 & -2.483882 & -0.301822 & $\mathrm{O}$ & -3.19482 & -2.48388 & -0.30182 \\
\hline $\mathrm{O}$ & 6.158861 & -1.729271 & -1.545611 & $\mathrm{O}$ & 6.158861 & -1.72927 & -1.54561 \\
\hline $\mathrm{H}$ & 2.6615 & 3.991675 & -1.163349 & $\mathrm{H}$ & 2.6615 & 3.991675 & -1.16335 \\
\hline $\mathrm{H}$ & 2.092809 & 2.239653 & -1.093396 & $\mathrm{H}$ & 2.092809 & 2.239653 & -1.0934 \\
\hline $\mathrm{H}$ & -0.636352 & -2.820239 & 1.001952 & $\mathrm{H}$ & -0.63635 & -2.82024 & 1.001952 \\
\hline $\mathrm{H}$ & -0.695756 & -2.721749 & -0.744432 & $\mathrm{H}$ & -0.69576 & -2.72175 & -0.74443 \\
\hline $\mathrm{H}$ & 1.530243 & -3.597691 & -0.320418 & $\mathrm{H}$ & 1.530243 & -3.59769 & -0.32042 \\
\hline $\mathrm{H}$ & 2.281812 & -2.965199 & 1.122587 & $\mathrm{H}$ & 2.281812 & -2.9652 & 1.122587 \\
\hline $\mathrm{H}$ & 3.836896 & -3.266317 & -0.867986 & $\mathrm{H}$ & 3.836896 & -3.26632 & -0.86799 \\
\hline $\mathrm{H}$ & 2.872563 & -2.107552 & -1.767748 & $\mathrm{H}$ & 2.872563 & -2.10755 & -1.76775 \\
\hline $\mathrm{H}$ & 5.809256 & 1.264296 & -1.794891 & $\mathrm{H}$ & 5.809256 & 1.264296 & -1.79489 \\
\hline $\mathrm{H}$ & 6.556737 & 0.524228 & -0.378142 & $\mathrm{H}$ & 6.556737 & 0.524228 & -0.37814 \\
\hline $\mathrm{H}$ & 3.054576 & -0.221698 & 2.085049 & $\mathrm{H}$ & 3.054576 & -0.2217 & 2.085049 \\
\hline $\mathrm{H}$ & 2.295776 & 1.232748 & 1.489908 & $\mathrm{H}$ & 2.295776 & 1.232748 & 1.489908 \\
\hline $\mathrm{H}$ & 4.881969 & 1.267521 & 1.010451 & $\mathrm{H}$ & 4.881969 & 1.267521 & 1.010451 \\
\hline $\mathrm{H}$ & 1.012288 & -1.038576 & -0.843398 & $\mathrm{H}$ & 1.012288 & -1.03858 & -0.8434 \\
\hline $\mathrm{H}$ & 2.747827 & 0.284221 & -0.905228 & $\mathrm{H}$ & 2.747827 & 0.284221 & -0.90523 \\
\hline $\mathrm{H}$ & 4.546411 & -0.672084 & -2.291343 & $\mathrm{H}$ & 4.546411 & -0.67208 & -2.29134 \\
\hline $\mathrm{H}$ & 5.387353 & 3.702854 & 0.942807 & $\mathrm{H}$ & 5.387353 & 3.702854 & 0.942807 \\
\hline $\mathrm{H}$ & 6.058715 & 3.445278 & -0.66254 & $\mathrm{H}$ & 6.058715 & 3.445278 & -0.66254 \\
\hline $\mathrm{H}$ & 4.793627 & 4.666952 & -0.426139 & $\mathrm{H}$ & 4.793627 & 4.666952 & -0.42614 \\
\hline
\end{tabular}




\begin{tabular}{|l|r|r|r|l|r|r|r|}
\hline $\mathrm{H}$ & -2.108473 & 3.423681 & -0.404241 & $\mathrm{H}$ & -2.10847 & 3.423681 & -0.40424 \\
\hline $\mathrm{H}$ & -1.307085 & 2.201746 & -1.411784 & $\mathrm{H}$ & -1.30709 & 2.201746 & -1.41178 \\
\hline $\mathrm{H}$ & -0.449678 & 2.895895 & -0.020035 & $\mathrm{H}$ & -0.44968 & 2.895895 & -0.02004 \\
\hline $\mathrm{H}$ & -2.782414 & 2.890379 & 1.993994 & $\mathrm{H}$ & -2.78241 & 2.890379 & 1.993994 \\
\hline $\mathrm{H}$ & -1.136392 & 2.308535 & 2.356627 & $\mathrm{H}$ & -1.13639 & 2.308535 & 2.356627 \\
\hline $\mathrm{H}$ & -2.539203 & 1.262354 & 2.663509 & $\mathrm{H}$ & -2.5392 & 1.262354 & 2.663509 \\
\hline $\mathrm{H}$ & 5.523639 & -0.89779 & 1.436651 & $\mathrm{H}$ & 5.523639 & -0.89779 & 1.436651 \\
\hline $\mathrm{H}$ & 5.596404 & -2.505261 & 0.710357 & $\mathrm{H}$ & 5.596404 & -2.50526 & 0.710357 \\
\hline $\mathrm{H}$ & 4.252644 & -2.075133 & 1.772284 & $\mathrm{H}$ & 4.252644 & -2.07513 & 1.772284 \\
\hline $\mathrm{H}$ & -0.298716 & -1.292598 & 2.701945 & $\mathrm{H}$ & -0.29872 & -1.2926 & 2.701945 \\
\hline $\mathrm{H}$ & 0.924874 & -0.180982 & 3.339296 & $\mathrm{H}$ & 0.924874 & -0.18098 & 3.339296 \\
\hline $\mathrm{H}$ & 1.374697 & -1.838888 & 2.907975 & $\mathrm{H}$ & 1.374697 & -1.83889 & 2.907975 \\
\hline $\mathrm{H}$ & -8.007097 & -1.098981 & -0.692063 & $\mathrm{H}$ & -8.0071 & -1.09898 & -0.69206 \\
\hline $\mathrm{H}$ & -7.667042 & -0.661537 & 0.989206 & $\mathrm{H}$ & -7.66704 & -0.66154 & 0.989206 \\
\hline $\mathrm{H}$ & -8.534795 & 0.473687 & -0.060303 & $\mathrm{H}$ & -8.5348 & 0.473687 & -0.0603 \\
\hline $\mathrm{H}$ & -6.695268 & 0.115568 & -2.62224 & $\mathrm{H}$ & -6.69527 & 0.115568 & -2.62224 \\
\hline $\mathrm{H}$ & -5.592171 & 1.448208 & -2.199155 & $\mathrm{H}$ & -5.59217 & 1.448208 & -2.19916 \\
\hline $\mathrm{H}$ & -7.341074 & 1.639461 & -1.98204 & $\mathrm{H}$ & -7.34107 & 1.639461 & -1.98204 \\
\hline $\mathrm{H}$ & -6.181857 & 1.224448 & 0.184242 & $\mathrm{H}$ & -6.18186 & 1.224448 & 0.184242 \\
\hline $\mathrm{H}$ & -4.210103 & -2.485116 & -0.482568 & $\mathrm{H}$ & -4.2101 & -2.48512 & -0.48257 \\
\hline $\mathrm{H}$ & 6.681767 & -1.443729 & -2.31136 & $\mathrm{H}$ & 6.681767 & -1.44373 & -2.31136 \\
\hline
\end{tabular}

Key transitions, oscillator strengths, and rotatory strengths in the ECD of conformers 3a (2) at B3LYP /6-311++G (d,p) level.

\begin{tabular}{|c|c|c|c|c|c|}
\hline Species & Excited State & $\Delta E(\mathrm{eV})^{\mathrm{a}}$ & $\lambda(\mathrm{nm})^{\mathrm{b}}$ & $f^{e}$ & $R_{\text {vel }}{ }^{\mathrm{d}}$ \\
\hline \multirow[t]{17}{*}{$\mathbf{3 a}(2)$} & $122->125$ & 3.7484 & 330.76 & 0.4023 & -13.1576 \\
\hline & $121->125$ & 3.9401 & 314.68 & 0.0147 & 28.3482 \\
\hline & $121->126$ & 4.2001 & 295.19 & 0.0011 & 1.0259 \\
\hline & $121->125$ & 4.3646 & 284.07 & 0.0011 & -5.9002 \\
\hline & $122->125$ & 4.4179 & 280.64 & 0.0001 & -0.0814 \\
\hline & $124->125$ & 4.5189 & 274.37 & 0.1339 & -5.9894 \\
\hline & $122->126$ & 5.0204 & 246.96 & 0.0008 & 6.3276 \\
\hline & $119->126$ & 5.2011 & 238.38 & 0.0649 & 9.778 \\
\hline & $119->125$ & 5.2359 & 236.8 & 0.0022 & 0.2736 \\
\hline & $122->126$ & 5.2601 & 235.7 & 0.0003 & 0.3951 \\
\hline & $124->132$ & 5.4021 & 229.51 & 0.0001 & 0.1957 \\
\hline & $115->125$ & 5.5852 & 221.99 & 0.0027 & 6.9072 \\
\hline & $116->125$ & 5.6093 & 221.03 & 0.0026 & -1.7225 \\
\hline & $124->127$ & 5.703 & 217.4 & 0.0738 & -18.8958 \\
\hline & $124->128$ & 5.7085 & 217.19 & 0.1112 & -10.2763 \\
\hline & $124->127$ & 5.7676 & 214.97 & 0.0008 & -0.2608 \\
\hline & $116->125$ & 5.8449 & 212.12 & 0.0065 & 1.7927 \\
\hline
\end{tabular}




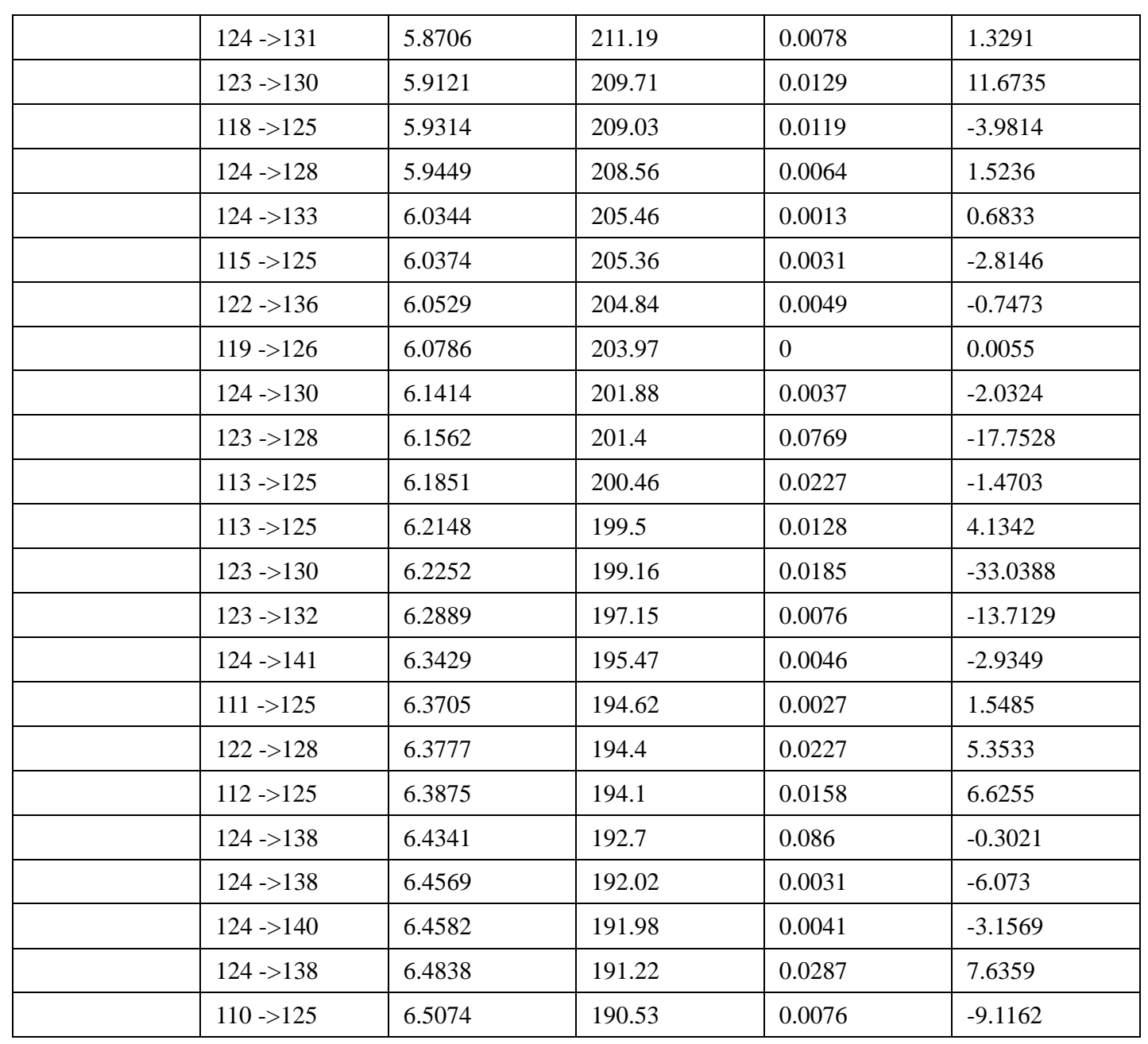

${ }^{\mathrm{a}}$ Excitation energy. ${ }^{\mathrm{b}}$ Wavelength. ${ }^{\mathrm{c}}$ Oscillator strength. ${ }^{\mathrm{d}}$ Rotatory strength in velocity form $(10-40 \mathrm{cgs}$.$) .$ 


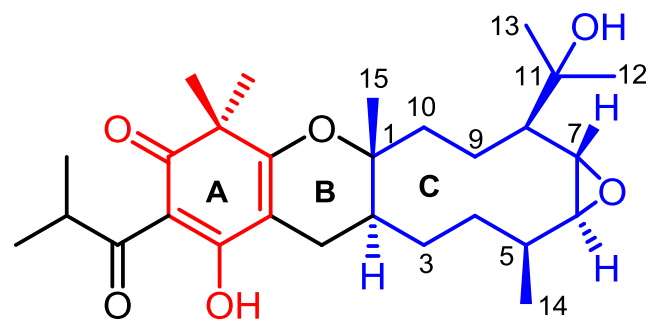

4A

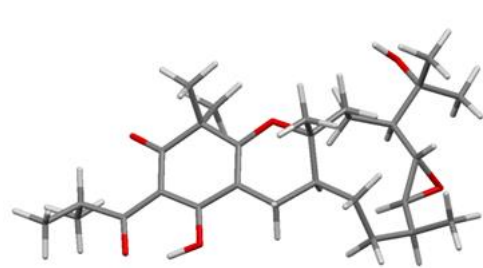

4A (1)

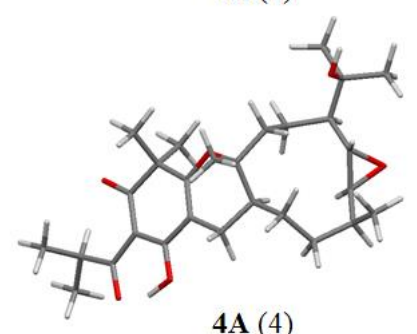

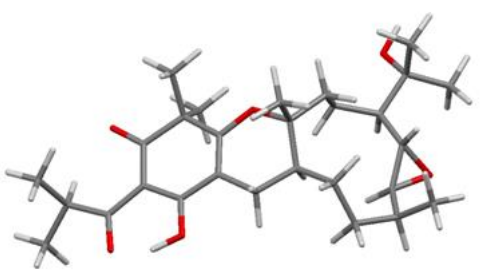

4A (2)

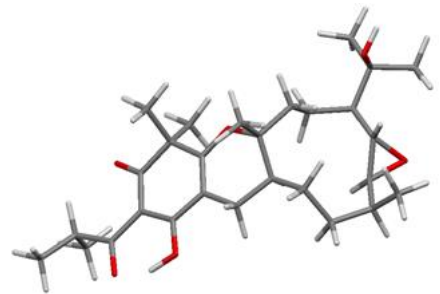

4A (3)

Optimized geometries of predominant conformers for compound $4 \mathrm{~A}$ at the B3LYP/6-31G (d,p) level in methanol solution.

Important thermodynamic parameters (a.u.) and Boltzmann distributions of the optimized compound 4A at B3LYP/6-31G(d,p) levelin methanol solution

\begin{tabular}{cccc}
\hline Conformation & E+ZPE & G & $\%$ \\
\hline $\mathbf{4 A}(1)$ & -1542.946020 & -1543.011414 & 2.19 \\
$\mathbf{4 A}(2)$ & -1542.946770 & -1543.012463 & 6.64 \\
$\mathbf{4 A}(3)$ & -1542.948338 & -1543.013744 & 25.76 \\
$\mathbf{4 A}(4)$ & -1542.948361 & -1543.014624 & 65.41
\end{tabular}

E+ZPE, G: total energy with zero point energy (ZPE) and Gibbs free energy in methanol solution at B3LYP/6-31G $(\mathrm{d}, \mathrm{p})$ level. \%: Boltzmann distributions, using the relative Gibbs free energies as weighting factors.

Optimized Z-matrixes of compound $\mathbf{4 A}$ in methanol solution $(\AA)$ at B3LYP/6-31G (d,p) level.

\begin{tabular}{|c|c|c|c|c|c|c|c|}
\hline \multicolumn{5}{|c|}{ 4A $(1)$} & \multicolumn{4}{|c|}{ 4A $(2)$} \\
\hline $\mathrm{C}$ & 3.996571 & 0.139418 & -0.134848 & $\mathrm{C}$ & 3.990481 & 0.156117 & -0.184256 \\
\hline $\mathrm{C}$ & 1.628073 & 0.766959 & 0.498665 & $\mathrm{C}$ & 1.625216 & 0.742322 & 0.503863 \\
\hline $\mathrm{C}$ & 2.971691 & 1.095274 & 0.121116 & $\mathrm{C}$ & 2.956622 & 1.093654 & 0.104944 \\
\hline $\mathrm{C}$ & 1.298424 & -0.542664 & 0.675539 & $\mathrm{C}$ & 1.303127 & -0.57519 & 0.629774 \\
\hline $\mathrm{C}$ & 3.645489 & -1.271958 & -0.074334 & $\mathrm{C}$ & 3.689778 & -1.260473 & -0.040018 \\
\hline $\mathrm{C}$ & 5.330668 & 0.631128 & -0.45152 & $\mathrm{C}$ & 5.283887 & 0.672352 & -0.61435 \\
\hline $\mathrm{C}$ & 0.607063 & 1.85635 & 0.703299 & $\mathrm{C}$ & 0.601153 & 1.816677 & 0.764707 \\
\hline
\end{tabular}




\begin{tabular}{|c|c|c|c|c|c|c|c|}
\hline $\mathrm{C}$ & -1.851147 & 2.403787 & 1.018147 & $\mathrm{C}$ & -1.854379 & 2.338735 & 1.130222 \\
\hline $\mathrm{C}$ & -2.248038 & 3.307502 & -0.176668 & $\mathrm{C}$ & -2.259305 & 3.30399 & -0.012409 \\
\hline $\mathrm{C}$ & -2.478591 & -1.266386 & -0.20398 & $\mathrm{C}$ & -2.482521 & -1.277956 & -0.259076 \\
\hline $\mathrm{C}$ & -2.265406 & -0.765172 & 1.248536 & $\mathrm{C}$ & -2.256447 & -0.840551 & 1.212885 \\
\hline $\mathrm{C}$ & -0.828026 & 1.302239 & 0.643871 & $\mathrm{C}$ & -0.832315 & 1.259784 & 0.693247 \\
\hline $\mathrm{C}$ & -3.613641 & 2.986785 & -0.836378 & $\mathrm{C}$ & -3.632964 & 3.018671 & -0.671144 \\
\hline $\mathrm{C}$ & -3.859689 & -1.014938 & -0.867688 & $\mathrm{C}$ & -3.861551 & -0.974087 & -0.907229 \\
\hline $\mathrm{C}$ & -3.67031 & 1.558201 & -1.365329 & $\mathrm{C}$ & -3.694506 & 1.618514 & -1.270185 \\
\hline $\mathrm{C}$ & -4.319077 & 0.42447 & -0.680705 & $\mathrm{C}$ & -4.327723 & 0.448069 & -0.635287 \\
\hline $\mathrm{C}$ & 2.251645 & -1.693077 & 0.461194 & $\mathrm{C}$ & 2.251459 & -1.710507 & 0.331579 \\
\hline $\mathrm{C}$ & -0.954013 & 0.012694 & 1.489713 & $\mathrm{C}$ & -0.947899 & -0.066846 & 1.481052 \\
\hline $\mathrm{C}$ & -4.799155 & 3.353269 & 0.068677 & $\mathrm{C}$ & -4.807687 & 3.338022 & 0.265233 \\
\hline $\mathrm{C}$ & 2.500258 & -2.390959 & 1.827786 & $\mathrm{C}$ & 2.34943 & -2.637499 & 1.569432 \\
\hline $\mathrm{C}$ & 1.630752 & -2.702888 & -0.53468 & $\mathrm{C}$ & 1.704019 & -2.51233 & -0.881167 \\
\hline $\mathrm{C}$ & -0.744639 & 0.25389 & 2.988084 & $\mathrm{C}$ & -0.731582 & 0.109991 & 2.987425 \\
\hline $\mathrm{C}$ & 7.832345 & 0.364903 & -0.243264 & $\mathrm{C}$ & 7.224068 & -0.574983 & 0.335557 \\
\hline $\mathrm{C}$ & 6.5635 & -0.654162 & -2.19632 & $\mathrm{C}$ & 7.392627 & 0.443048 & -1.986091 \\
\hline $\mathrm{C}$ & -6.232962 & -1.794562 & -1.338966 & $\mathrm{C}$ & -6.255734 & -1.704737 & -1.374529 \\
\hline $\mathrm{C}$ & -5.315664 & -2.127295 & 0.981333 & $\mathrm{C}$ & -5.265292 & -2.22009 & 0.885611 \\
\hline $\mathrm{C}$ & 6.51974 & -0.289413 & -0.695885 & $\mathrm{C}$ & 6.463882 & -0.225628 & -0.963737 \\
\hline $\mathrm{C}$ & -4.96858 & -2.060748 & -0.513291 & $\mathrm{C}$ & -4.963109 & -2.033781 & -0.609274 \\
\hline $\mathrm{O}$ & 4.404904 & -2.190564 & -0.402503 & $\mathrm{O}$ & 4.514428 & -2.16687 & -0.205059 \\
\hline $\mathrm{O}$ & 5.544823 & 1.87236 & -0.535543 & $\mathrm{O}$ & 5.471318 & 1.917719 & -0.700275 \\
\hline $\mathrm{O}$ & 0.087487 & -0.951669 & 1.066673 & $\mathrm{O}$ & 0.097855 & -1.006176 & 1.012949 \\
\hline $\mathrm{O}$ & -4.951372 & 1.066358 & -1.810623 & $\mathrm{O}$ & -4.980693 & 1.145613 & -1.720181 \\
\hline $\mathrm{O}$ & 3.217941 & 2.387336 & 0.023139 & $\mathrm{O}$ & 3.182069 & 2.390192 & 0.017176 \\
\hline $\mathrm{O}$ & -4.522772 & -3.362138 & -0.949988 & $\mathrm{O}$ & -4.414445 & -3.261397 & -1.134026 \\
\hline $\mathrm{H}$ & 0.777205 & 2.366031 & 1.662047 & $\mathrm{H}$ & 0.777515 & 2.284088 & 1.743789 \\
\hline $\mathrm{H}$ & 0.731027 & 2.626586 & -0.06531 & $\mathrm{H}$ & 0.715262 & 2.620368 & 0.029855 \\
\hline $\mathrm{H}$ & -1.4196 & 3.020504 & 1.815238 & $\mathrm{H}$ & -1.419666 & 2.911416 & 1.957803 \\
\hline $\mathrm{H}$ & -2.753396 & 1.961305 & 1.451401 & $\mathrm{H}$ & -2.753813 & 1.871323 & 1.542549 \\
\hline $\mathrm{H}$ & -2.288842 & 4.352898 & 0.151442 & $\mathrm{H}$ & -2.292555 & 4.331732 & 0.368106 \\
\hline $\mathrm{H}$ & -1.461484 & 3.267446 & -0.942032 & $\mathrm{H}$ & -1.48178 & 3.299405 & -0.787988 \\
\hline $\mathrm{H}$ & -1.732622 & -0.804573 & -0.857193 & $\mathrm{H}$ & -1.727035 & -0.805754 & -0.894201 \\
\hline $\mathrm{H}$ & -2.25382 & -2.337097 & -0.255609 & $\mathrm{H}$ & -2.304169 & -2.352313 & -0.350526 \\
\hline $\mathrm{H}$ & -3.088073 & -0.125753 & 1.57872 & $\mathrm{H}$ & -3.079877 & -0.224515 & 1.5836 \\
\hline $\mathrm{H}$ & -2.260839 & -1.616214 & 1.938209 & $\mathrm{H}$ & -2.238417 & -1.72323 & 1.86147 \\
\hline $\mathrm{H}$ & -1.008321 & 1.007046 & -0.396273 & $\mathrm{H}$ & -1.022856 & 1.012241 & -0.357449 \\
\hline $\mathrm{H}$ & -3.680686 & 3.629584 & -1.727284 & $\mathrm{H}$ & -3.710819 & 3.705193 & -1.527866 \\
\hline $\mathrm{H}$ & -3.700946 & -1.143771 & -1.94608 & $\mathrm{H}$ & -3.70578 & -1.037683 & -1.992073 \\
\hline $\mathrm{H}$ & -2.863162 & 1.311647 & -2.060456 & $\mathrm{H}$ & -2.897596 & 1.410734 & -1.989326 \\
\hline $\mathrm{H}$ & -4.830948 & 0.645024 & 0.25538 & $\mathrm{H}$ & -4.826345 & 0.614696 & 0.31913 \\
\hline
\end{tabular}




\begin{tabular}{|c|c|c|c|c|c|c|c|}
\hline $\mathrm{H}$ & -4.747734 & 2.860522 & 1.046228 & $\mathrm{H}$ & -4.744299 & 2.797089 & 1.216252 \\
\hline $\mathrm{H}$ & -5.746169 & 3.07109 & -0.40105 & $\mathrm{H}$ & -5.760191 & 3.078588 & -0.206498 \\
\hline $\mathrm{H}$ & -4.813304 & 4.433955 & 0.246433 & $\mathrm{H}$ & -4.820265 & 4.408453 & 0.49708 \\
\hline $\mathrm{H}$ & 2.940504 & -1.698032 & 2.553154 & $\mathrm{H}$ & 2.704241 & -2.086359 & 2.447519 \\
\hline $\mathrm{H}$ & 3.190524 & -3.225557 & 1.680035 & $\mathrm{H}$ & 3.057277 & -3.441103 & 1.356367 \\
\hline $\mathrm{H}$ & 1.558768 & -2.768845 & 2.234934 & $\mathrm{H}$ & 1.372057 & -3.066598 & 1.801946 \\
\hline $\mathrm{H}$ & 0.705985 & -3.119822 & -0.129128 & $\mathrm{H}$ & 0.720392 & -2.928159 & -0.647856 \\
\hline $\mathrm{H}$ & 2.344526 & -3.50975 & -0.711736 & $\mathrm{H}$ & 2.396024 & -3.327129 & -1.108265 \\
\hline $\mathrm{H}$ & 1.404167 & -2.222757 & -1.493035 & $\mathrm{H}$ & 1.611204 & -1.87556 & -1.767847 \\
\hline $\mathrm{H}$ & -0.771498 & -0.697116 & 3.528533 & $\mathrm{H}$ & -0.754433 & -0.863176 & 3.487043 \\
\hline $\mathrm{H}$ & -1.535757 & 0.896812 & 3.386368 & $\mathrm{H}$ & -1.521698 & 0.734006 & 3.416496 \\
\hline $\mathrm{H}$ & 0.217869 & 0.733682 & 3.188036 & $\mathrm{H}$ & 0.231304 & 0.582123 & 3.203456 \\
\hline $\mathrm{H}$ & 7.80462 & 0.624996 & 0.820931 & $\mathrm{H}$ & 6.582081 & -1.11101 & 1.039775 \\
\hline $\mathrm{H}$ & 8.662566 & -0.332951 & -0.399328 & $\mathrm{H}$ & 8.081929 & -1.215544 & 0.101785 \\
\hline $\mathrm{H}$ & 8.041143 & 1.278799 & -0.807011 & $\mathrm{H}$ & 7.600452 & 0.333312 & 0.821007 \\
\hline $\mathrm{H}$ & 5.653908 & -1.178591 & -2.50169 & $\mathrm{H}$ & 6.858729 & 0.694024 & -2.909466 \\
\hline $\mathrm{H}$ & 7.418251 & -1.310906 & -2.393624 & $\mathrm{H}$ & 8.208545 & -0.24205 & -2.242036 \\
\hline $\mathrm{H}$ & 6.676435 & 0.246219 & -2.811726 & $\mathrm{H}$ & 7.829169 & 1.364702 & -1.590141 \\
\hline $\mathrm{H}$ & -6.969402 & -2.581591 & -1.146112 & $\mathrm{H}$ & -6.717018 & -0.778056 & -1.019052 \\
\hline $\mathrm{H}$ & -5.999725 & -1.79421 & -2.408908 & $\mathrm{H}$ & -6.984083 & -2.514333 & -1.241188 \\
\hline $\mathrm{H}$ & -6.680088 & -0.829084 & -1.086929 & $\mathrm{H}$ & -6.051985 & -1.598857 & -2.444819 \\
\hline $\mathrm{H}$ & -4.44798 & -2.417834 & 1.584282 & $\mathrm{H}$ & -4.378874 & -2.558638 & 1.428546 \\
\hline $\mathrm{H}$ & -5.683477 & -1.168724 & 1.362666 & $\mathrm{H}$ & -5.630325 & -1.298257 & 1.351761 \\
\hline $\mathrm{H}$ & -6.099574 & -2.875386 & 1.140057 & $\mathrm{H}$ & -6.047737 & -2.978832 & 1.013006 \\
\hline $\mathrm{H}$ & 6.356682 & -1.214263 & -0.140679 & $\mathrm{H}$ & 6.072448 & -1.158526 & -1.37229 \\
\hline $\mathrm{H}$ & 4.213683 & 2.455122 & -0.237808 & $\mathrm{H}$ & 4.164419 & 2.475123 & -0.287302 \\
\hline $\mathrm{H}$ & -3.872171 & -3.685785 & -0.306976 & $\mathrm{H}$ & -5.052099 & -3.967299 & -0.937488 \\
\hline \multicolumn{4}{|c|}{ 4A (3) } & \multicolumn{4}{|c|}{ 4A (4) } \\
\hline $\mathrm{C}$ & 4.018317 & 0.143326 & -0.110676 & $\mathrm{C}$ & 4.018026 & 0.161283 & -0.147414 \\
\hline $\mathrm{C}$ & 1.640011 & 0.743583 & 0.514025 & $\mathrm{C}$ & 1.640926 & 0.717148 & 0.526232 \\
\hline $\mathrm{C}$ & 2.989738 & 1.087295 & 0.175157 & $\mathrm{C}$ & 2.982454 & 1.084874 & 0.179248 \\
\hline $\mathrm{C}$ & 1.306287 & -0.573202 & 0.622392 & $\mathrm{C}$ & 1.311463 & -0.604604 & 0.564593 \\
\hline $\mathrm{C}$ & 3.665808 & -1.268761 & -0.122746 & $\mathrm{C}$ & 3.710631 & -1.259909 & -0.091052 \\
\hline $\mathrm{C}$ & 5.356838 & 0.647773 & -0.384932 & $\mathrm{C}$ & 5.319119 & 0.695844 & -0.528306 \\
\hline $\mathrm{C}$ & 0.614681 & 1.823845 & 0.747118 & $\mathrm{C}$ & 0.612178 & 1.78009 & 0.817876 \\
\hline $\mathrm{C}$ & -1.857039 & 2.351563 & 1.01572 & $\mathrm{C}$ & -1.860441 & 2.284103 & 1.118844 \\
\hline $\mathrm{C}$ & -2.242488 & 3.280703 & -0.164796 & $\mathrm{C}$ & -2.24818 & 3.27343 & -0.011034 \\
\hline $\mathrm{C}$ & -2.491499 & -1.228089 & -0.330905 & $\mathrm{C}$ & -2.502929 & -1.216445 & -0.400879 \\
\hline $\mathrm{C}$ & -2.26913 & -0.811369 & 1.144016 & $\mathrm{C}$ & -2.262567 & -0.881305 & 1.091784 \\
\hline $\mathrm{C}$ & -0.816355 & 1.269283 & 0.633229 & $\mathrm{C}$ & -0.81712 & 1.226952 & 0.678736 \\
\hline $\mathrm{C}$ & -3.611623 & 2.988476 & -0.831894 & $\mathrm{C}$ & -3.62447 & 3.021858 & -0.680397 \\
\hline $\mathrm{C}$ & -3.912536 & -1.010246 & -0.91154 & $\mathrm{C}$ & -3.931797 & -0.968309 & -0.950045 \\
\hline
\end{tabular}




\begin{tabular}{|c|c|c|c|c|c|c|c|}
\hline $\mathrm{C}$ & -3.6851 & 1.569613 & -1.385561 & $\mathrm{C}$ & -3.709094 & 1.631582 & -1.301181 \\
\hline $\mathrm{C}$ & -4.355996 & 0.437052 & -0.723946 & $\mathrm{C}$ & -4.370531 & 0.468412 & -0.68468 \\
\hline $\mathrm{C}$ & 2.263313 & -1.712809 & 0.369411 & $\mathrm{C}$ & 2.263644 & -1.724458 & 0.223122 \\
\hline $\mathrm{C}$ & -0.957268 & -0.052954 & 1.426202 & $\mathrm{C}$ & -0.950268 & -0.135049 & 1.402111 \\
\hline $\mathrm{C}$ & -4.793228 & 3.352168 & 0.07919 & $\mathrm{C}$ & -4.796406 & 3.346074 & 0.25762 \\
\hline $\mathrm{C}$ & 2.48939 & -2.474368 & 1.705497 & $\mathrm{C}$ & 2.332083 & -2.720063 & 1.408655 \\
\hline $\mathrm{C}$ & 1.658329 & -2.673869 & -0.682826 & $\mathrm{C}$ & 1.740574 & -2.454105 & -1.044514 \\
\hline $\mathrm{C}$ & -0.759988 & 0.127687 & 2.934565 & $\mathrm{C}$ & -0.742468 & -0.033768 & 2.916827 \\
\hline $\mathrm{C}$ & 7.856196 & 0.36986 & -0.162933 & $\mathrm{C}$ & 7.251244 & -0.615932 & 0.349226 \\
\hline $\mathrm{C}$ & 6.60837 & -0.55611 & -2.1747 & $\mathrm{C}$ & 7.429388 & 0.539177 & -1.907058 \\
\hline $\mathrm{C}$ & -4.584988 & -3.465691 & -0.60504 & $\mathrm{C}$ & -4.603446 & -3.435568 & -0.756312 \\
\hline $\mathrm{C}$ & -6.337011 & -1.747771 & -1.137521 & $\mathrm{C}$ & -6.360614 & -1.691556 & -1.177359 \\
\hline $\mathrm{C}$ & 6.548201 & -0.261485 & -0.659408 & $\mathrm{C}$ & 6.4958 & -0.18537 & -0.9281 \\
\hline $\mathrm{C}$ & -5.004181 & -2.000089 & -0.411006 & $\mathrm{C}$ & -5.01773 & -1.981123 & -0.484017 \\
\hline $\mathrm{O}$ & 4.430102 & -2.171902 & -0.481578 & $\mathrm{O}$ & 4.536768 & -2.158514 & -0.288583 \\
\hline $\mathrm{O}$ & 5.573543 & 1.891343 & -0.404923 & $\mathrm{O}$ & 5.515421 & 1.942897 & -0.529944 \\
\hline $\mathrm{O}$ & 0.089319 & -0.999541 & 0.971586 & $\mathrm{O}$ & 0.095853 & -1.052569 & 0.89103 \\
\hline $\mathrm{O}$ & -4.967914 & 1.102629 & -1.854179 & $\mathrm{O}$ & -4.999225 & 1.187977 & -1.771997 \\
\hline $\mathrm{O}$ & 3.238855 & 2.382287 & 0.14287 & $\mathrm{O}$ & 3.215462 & 2.383073 & 0.175646 \\
\hline $\mathrm{O}$ & -5.170918 & -1.733925 & 0.997571 & $\mathrm{O}$ & -5.163974 & -1.785716 & 0.938454 \\
\hline $\mathrm{H}$ & 0.764724 & 2.291432 & 1.730393 & $\mathrm{H}$ & 0.762458 & 2.196331 & 1.823977 \\
\hline $\mathrm{H}$ & 0.752469 & 2.626732 & 0.014989 & $\mathrm{H}$ & 0.745165 & 2.620156 & 0.128085 \\
\hline $\mathrm{H}$ & -1.447695 & 2.952329 & 1.836496 & $\mathrm{H}$ & -1.45289 & 2.840277 & 1.971261 \\
\hline $\mathrm{H}$ & -2.761888 & 1.887333 & 1.419158 & $\mathrm{H}$ & -2.764134 & 1.795927 & 1.495816 \\
\hline $\mathrm{H}$ & -2.270428 & 4.321233 & 0.179898 & $\mathrm{H}$ & -2.266265 & 4.296106 & 0.383948 \\
\hline $\mathrm{H}$ & -1.455933 & 3.24338 & -0.930463 & $\mathrm{H}$ & -1.46821 & 3.268152 & -0.7843 \\
\hline $\mathrm{H}$ & -1.809747 & -0.66989 & -0.979289 & $\mathrm{H}$ & -1.829572 & -0.622911 & -1.026309 \\
\hline $\mathrm{H}$ & -2.201138 & -2.274507 & -0.459769 & $\mathrm{H}$ & -2.214386 & -2.254169 & -0.590401 \\
\hline $\mathrm{H}$ & -3.092582 & -0.193091 & 1.505835 & $\mathrm{H}$ & -3.084268 & -0.288451 & 1.497388 \\
\hline $\mathrm{H}$ & -2.279529 & -1.701218 & 1.7822 & $\mathrm{H}$ & -2.261828 & -1.805253 & 1.679527 \\
\hline $\mathrm{H}$ & -0.967472 & 1.011671 & -0.421096 & $\mathrm{H}$ & -0.970289 & 1.024028 & -0.387296 \\
\hline $\mathrm{H}$ & -3.669607 & 3.648686 & -1.710776 & $\mathrm{H}$ & -3.686434 & 3.724165 & -1.525698 \\
\hline $\mathrm{H}$ & -3.83212 & -1.173025 & -1.994338 & $\mathrm{H}$ & -3.866914 & -1.076881 & -2.040696 \\
\hline $\mathrm{H}$ & -2.874316 & 1.324941 & -2.077361 & $\mathrm{H}$ & -2.90933 & 1.42033 & -2.016374 \\
\hline $\mathrm{H}$ & -4.877396 & 0.637425 & 0.209692 & $\mathrm{H}$ & -4.877623 & 0.623962 & 0.265258 \\
\hline $\mathrm{H}$ & -4.747374 & 2.840335 & 1.047117 & $\mathrm{H}$ & -4.743112 & 2.789729 & 1.200284 \\
\hline $\mathrm{H}$ & -5.74299 & 3.088657 & -0.396012 & $\mathrm{H}$ & -5.751575 & 3.107733 & -0.219975 \\
\hline $\mathrm{H}$ & -4.795844 & 4.429601 & 0.276662 & $\mathrm{H}$ & -4.793433 & 4.413158 & 0.505035 \\
\hline $\mathrm{H}$ & 2.9192 & -1.81673 & 2.469008 & $\mathrm{H}$ & 3.040876 & -3.514185 & 1.165323 \\
\hline $\mathrm{H}$ & 3.180703 & -3.302778 & 1.530033 & $\mathrm{H}$ & 1.348257 & -3.156109 & 1.596622 \\
\hline $\mathrm{H}$ & 1.541132 & -2.868752 & 2.079936 & $\mathrm{H}$ & 2.670956 & -2.220833 & 2.323362 \\
\hline $\mathrm{H}$ & 1.452447 & -2.14993 & -1.622765 & $\mathrm{H}$ & 0.752086 & -2.880876 & -0.855585 \\
\hline
\end{tabular}




\begin{tabular}{|c|c|c|c|c|c|c|c|}
\hline $\mathrm{H}$ & 0.724052 & -3.103637 & -0.314205 & $\mathrm{H}$ & 2.436201 & -3.255866 & -1.304577 \\
\hline $\mathrm{H}$ & 2.372409 & -3.47521 & -0.882048 & $\mathrm{H}$ & 1.666277 & -1.76674 & -1.89433 \\
\hline $\mathrm{H}$ & -0.814789 & -0.841534 & 3.43953 & $\mathrm{H}$ & -0.800584 & -1.027237 & 3.371826 \\
\hline $\mathrm{H}$ & -1.542508 & 0.772873 & 3.346188 & $\mathrm{H}$ & -1.518081 & 0.594375 & 3.366228 \\
\hline $\mathrm{H}$ & 0.20986 & 0.578251 & 3.164867 & $\mathrm{H}$ & 0.231831 & 0.397907 & 3.163888 \\
\hline $\mathrm{H}$ & 7.816978 & 0.581176 & 0.911623 & $\mathrm{H}$ & 7.632672 & 0.259337 & 0.888425 \\
\hline $\mathrm{H}$ & 8.68749 & -0.32123 & -0.341842 & $\mathrm{H}$ & 6.604177 & -1.188425 & 1.019317 \\
\hline $\mathrm{H}$ & 8.071835 & 1.308281 & -0.682047 & $\mathrm{H}$ & 8.105239 & -1.247404 & 0.079402 \\
\hline $\mathrm{H}$ & 5.704019 & -1.068876 & -2.513666 & $\mathrm{H}$ & 6.898143 & 0.847339 & -2.81455 \\
\hline $\mathrm{H}$ & 7.46761 & -1.200145 & -2.393345 & $\mathrm{H}$ & 8.242904 & -0.132786 & -2.202612 \\
\hline $\mathrm{H}$ & 6.724314 & 0.372184 & -2.74653 & $\mathrm{H}$ & 7.869193 & 1.433465 & -1.455895 \\
\hline $\mathrm{H}$ & -4.341771 & -3.676658 & -1.652824 & $\mathrm{H}$ & -5.42277 & -4.114248 & -0.489316 \\
\hline $\mathrm{H}$ & -5.406538 & -4.131475 & -0.313474 & $\mathrm{H}$ & -3.729917 & -3.718268 & -0.162574 \\
\hline $\mathrm{H}$ & -3.717865 & -3.716766 & 0.011837 & $\mathrm{H}$ & -4.372194 & -3.594641 & -1.815902 \\
\hline $\mathrm{H}$ & -6.686099 & -0.722464 & -0.987771 & $\mathrm{H}$ & -6.70299 & -0.672867 & -0.976166 \\
\hline $\mathrm{H}$ & -6.240157 & -1.923929 & -2.21469 & $\mathrm{H}$ & -6.281228 & -1.819529 & -2.262741 \\
\hline $\mathrm{H}$ & -7.106661 & -2.430463 & -0.755941 & $\mathrm{H}$ & -7.127187 & -2.387854 & -0.814668 \\
\hline $\mathrm{H}$ & 6.378892 & -1.211003 & -0.149542 & $\mathrm{H}$ & 6.101239 & -1.090317 & -1.39266 \\
\hline $\mathrm{H}$ & 4.238421 & 2.460725 & -0.099263 & $\mathrm{H}$ & 4.203913 & 2.481008 & -0.103776 \\
\hline $\mathrm{H}$ & -5.890288 & -2.303554 & 1.316269 & $\mathrm{H}$ & -5.875639 & -2.374383 & 1.239412 \\
\hline
\end{tabular}

Key transitions, oscillator strengths, and rotatory strengths in the ECD of conformers 4A (4) at B3LYP /6-311++G $(\mathrm{d}, \mathrm{p})$ level.

\begin{tabular}{|c|c|c|c|c|c|}
\hline Species & Excited State & $\Delta \mathrm{E}(\mathrm{eV})^{\mathrm{a}}$ & $\lambda(\mathrm{nm})^{\mathrm{b}}$ & $\mathrm{f}^{\mathrm{c}}$ & Rvel $^{\mathrm{d}}$ \\
\hline 4A (4) & $129->131$ & 3.7497 & 330.65 & 0.396 & -24.5442 \\
\hline & $128->131$ & 3.9538 & 313.58 & 0.0173 & 32.0679 \\
\hline & $128->131$ & 4.2067 & 294.73 & 0.0011 & -0.9672 \\
\hline & $128->144$ & 4.37 & 283.71 & 0.0023 & -7.832 \\
\hline & $125->130$ & 4.5094 & 274.95 & 0.1337 & -1.1876 \\
\hline & $126->131$ & 4.9669 & 249.62 & 0.0002 & 0.2021 \\
\hline & $126->130$ & 5.0238 & 246.79 & 0.001 & 8.7408 \\
\hline & $129->142$ & 5.223 & 237.38 & 0.0654 & 6.6601 \\
\hline & $123->130$ & 5.3474 & 231.86 & 0 & 0.4079 \\
\hline & $129->133$ & 5.3827 & 230.34 & 0.0002 & 0.3708 \\
\hline & $122->130$ & 5.4171 & 228.88 & 0.0027 & 3.4525 \\
\hline & $120->130$ & 5.6517 & 219.37 & 0.0022 & 4.3785 \\
\hline & $125>130$ & 5.7043 & 217.35 & 0.182 & -23.6794 \\
\hline & $129->136$ & 5.7237 & 216.61 & 0.0019 & 1.3549 \\
\hline & $129->134$ & 5.7945 & 213.97 & 0.0106 & 3.5503 \\
\hline & $128->131$ & 5.8047 & 213.59 & 0.0003 & 0.0585 \\
\hline & $129->138$ & 5.8255 & 212.83 & 0.0043 & 1.8945 \\
\hline
\end{tabular}




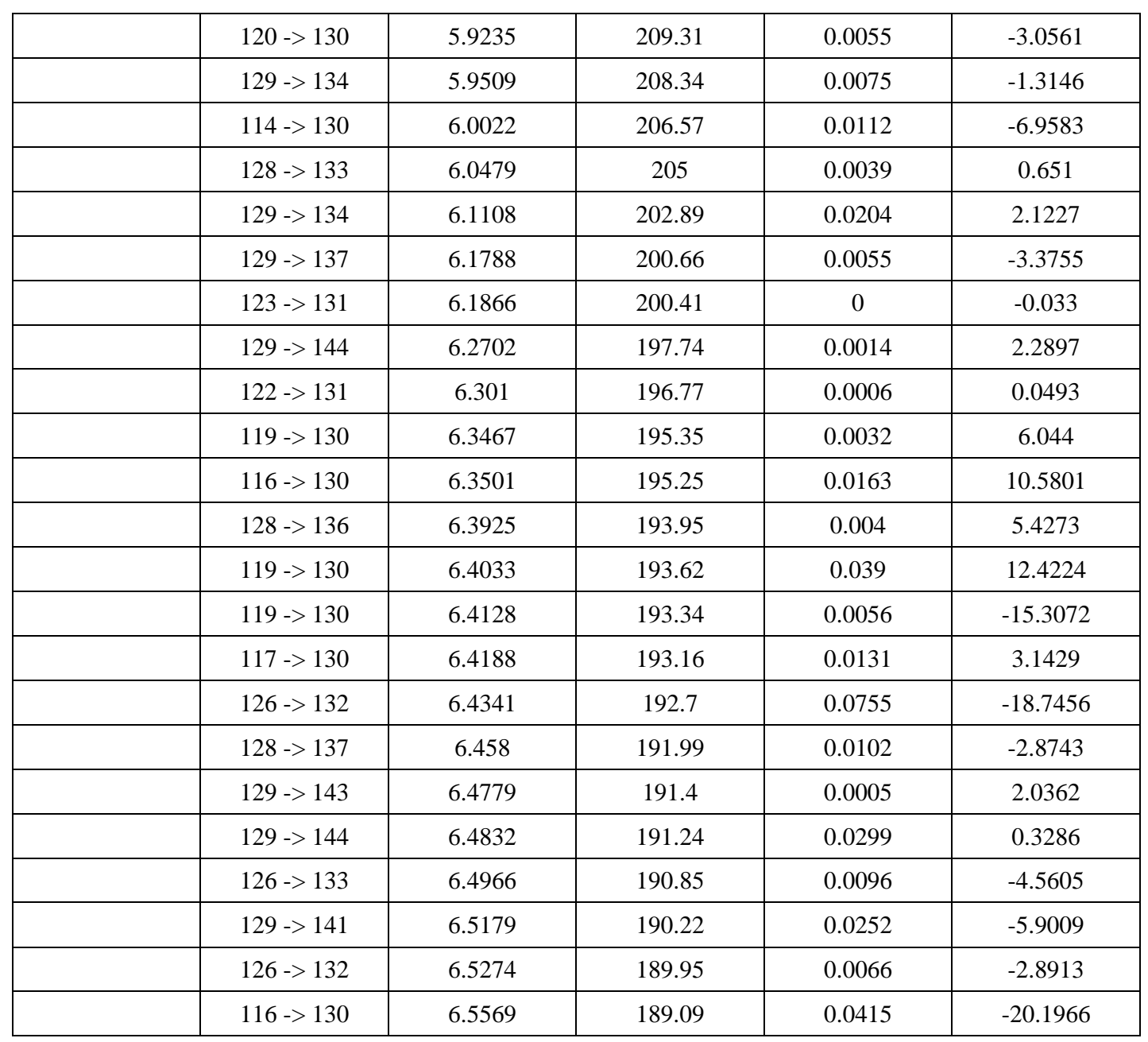

${ }^{\mathrm{a}}$ Excitation energy. ${ }^{\mathrm{b}}$ Wavelength. ${ }^{\mathrm{c}}$ Oscillator strength. ${ }^{\mathrm{d}}$ Rotatory strength in velocity form $(10-40 \mathrm{cgs}$.$) .$

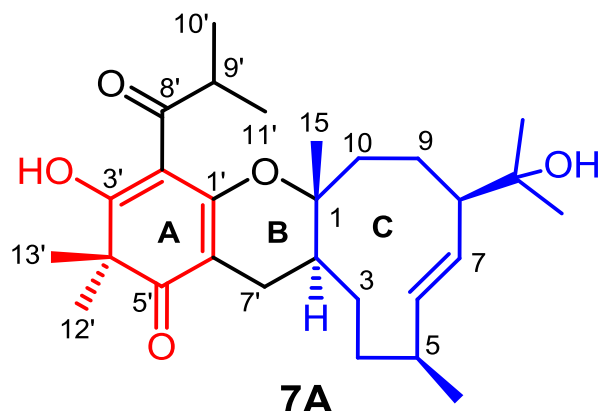

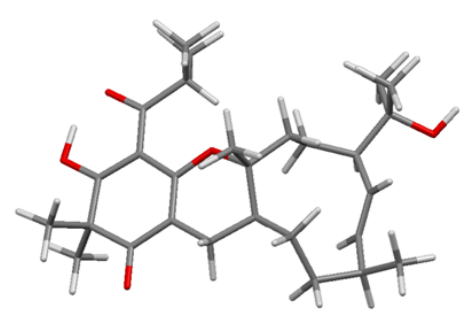

7A (1)

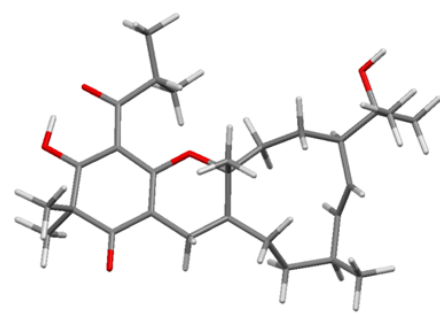

7A (2)

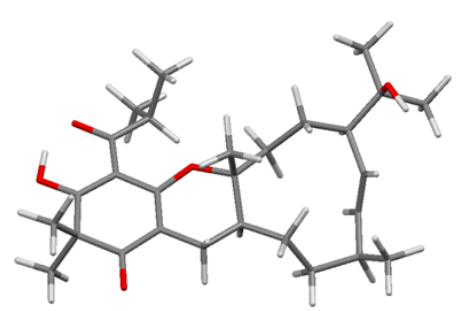

7A (3)

Optimized geometries of predominant conformers for compound 7A at the B3LYP/6-31G (d,p) level in methanol solution. 
Important thermodynamic parameters (a.u.) and Boltzmann distributions of the optimized compound 7A at B3LYP/6-31G (d,p) levelin methanol solution.

\begin{tabular}{llll}
\hline Conformation & E+ZPE & G & $\%$ \\
\hline 7A $(1)$ & -1467.731199 & -1467.795400 & 99.02 \\
7A $(2)$ & -1467.726342 & -1467.790651 & 0.65 \\
7A $(3)$ & -1467.725598 & -1467.790014 & 0.33 \\
\hline
\end{tabular}

E+ZPE, G: total energy with zero point energy (ZPE) and Gibbs free energy in methanol solution at B3LYP/6-31G $(\mathrm{d}, \mathrm{p})$ level. \%: Boltzmann distributions, using the relative Gibbs free energies as weighting factors.

Optimized Z-matrixes of compound 7A in methanol solution $(\AA)$ at B3LYP/6-31G (d,p) level

\begin{tabular}{|c|c|c|c|c|c|c|c|}
\hline \multicolumn{4}{|c|}{ 7A (1) } & \multicolumn{4}{|c|}{ 7A (2) } \\
\hline $\mathrm{C}$ & 3.430788 & -1.670418 & -1.255567 & $\mathrm{C}$ & 3.907293 & -1.411473 & -1.347316 \\
\hline $\mathrm{C}$ & 3.877722 & -0.592713 & -0.603525 & $\mathrm{C}$ & 4.323691 & -0.657339 & -0.32383 \\
\hline $\mathrm{C}$ & -2.87582 & 0.834242 & -0.047465 & $\mathrm{C}$ & -3.205049 & 0.852727 & -0.092329 \\
\hline $\mathrm{C}$ & -1.9376 & -1.49236 & 0.116901 & $\mathrm{C}$ & -2.196962 & -1.424942 & 0.265517 \\
\hline $\mathrm{C}$ & -1.802217 & -0.131203 & 0.251127 & $\mathrm{C}$ & -2.067302 & -0.06541 & 0.100395 \\
\hline $\mathrm{C}$ & -4.125033 & 0.29542 & -0.375065 & $\mathrm{C}$ & -4.481022 & 0.276797 & -0.061902 \\
\hline $\mathrm{C}$ & -3.188381 & -2.079062 & -0.28632 & $\mathrm{C}$ & -3.492392 & -2.048573 & 0.314094 \\
\hline $\mathrm{C}$ & -2.754266 & 2.298479 & -0.042936 & $\mathrm{C}$ & -3.127619 & 2.308747 & -0.291051 \\
\hline $\mathrm{C}$ & -0.789703 & -2.420777 & 0.410319 & $\mathrm{C}$ & -0.985694 & -2.312978 & 0.37731 \\
\hline $\mathrm{C}$ & 2.235791 & -3.591042 & -0.173773 & $\mathrm{C}$ & 2.156288 & -3.148828 & -1.112645 \\
\hline $\mathrm{C}$ & 1.981439 & 1.046372 & -0.369197 & $\mathrm{C}$ & 2.790281 & 0.968862 & 0.692691 \\
\hline $\mathrm{C}$ & 1.675302 & -2.667143 & 0.936346 & $\mathrm{C}$ & 1.550313 & -2.50216 & 0.165711 \\
\hline $\mathrm{C}$ & 1.674689 & 0.559591 & 1.073566 & $\mathrm{C}$ & 1.493379 & 0.683906 & -0.111405 \\
\hline $\mathrm{C}$ & 3.579254 & -3.106621 & -0.809365 & $\mathrm{C}$ & 3.689294 & -2.905711 & -1.306358 \\
\hline $\mathrm{C}$ & 3.42918 & 0.821835 & -0.910908 & $\mathrm{C}$ & 4.073904 & 0.821981 & -0.183503 \\
\hline $\mathrm{C}$ & 0.568539 & -1.704235 & 0.434177 & $\mathrm{C}$ & 0.297663 & -1.612281 & -0.083767 \\
\hline $\mathrm{C}$ & -4.41017 & -1.178909 & -0.52452 & $\mathrm{C}$ & -4.756668 & -1.188913 & 0.170332 \\
\hline $\mathrm{C}$ & 0.457788 & -0.381809 & 1.230444 & $\mathrm{C}$ & 0.367502 & -0.187755 & 0.523917 \\
\hline $\mathrm{C}$ & 4.764644 & -3.355835 & 0.134607 & $\mathrm{C}$ & 4.520408 & -3.652452 & -0.254321 \\
\hline $\mathrm{C}$ & -5.50008 & -1.579941 & 0.505859 & $\mathrm{C}$ & -5.575469 & -1.329218 & 1.482404 \\
\hline $\mathrm{C}$ & -4.929144 & -1.439464 & -1.962959 & $\mathrm{C}$ & -5.580836 & -1.740151 & -1.021975 \\
\hline $\mathrm{C}$ & 0.154517 & -0.605682 & 2.716649 & $\mathrm{C}$ & 0.349165 & -0.159966 & 2.054741 \\
\hline $\mathrm{C}$ & -1.299264 & 3.409417 & 1.652433 & $\mathrm{C}$ & -2.067483 & 4.58128 & 0.017865 \\
\hline $\mathrm{C}$ & -1.441138 & 4.346163 & -0.702402 & $\mathrm{C}$ & -1.289815 & 3.015792 & -1.822037 \\
\hline $\mathrm{C}$ & 4.672309 & 2.015725 & 1.031054 & $\mathrm{C}$ & 6.495672 & 1.458433 & -0.564547 \\
\hline $\mathrm{C}$ & 4.128549 & 3.269272 & -1.082722 & $\mathrm{C}$ & 5.658135 & 1.263873 & 1.808924 \\
\hline $\mathrm{C}$ & -1.4537 & 3.069787 & 0.153028 & $\mathrm{C}$ & -1.837293 & 3.116557 & -0.379309 \\
\hline $\mathrm{C}$ & 4.473199 & 1.894102 & -0.487888 & $\mathrm{C}$ & 5.283674 & 1.62731 & 0.363818 \\
\hline $\mathrm{O}$ & -3.328134 & -3.303357 & -0.424865 & $\mathrm{O}$ & -3.629922 & -3.270504 & 0.473984 \\
\hline $\mathrm{O}$ & -3.785224 & 2.999726 & -0.219485 & $\mathrm{O}$ & -4.193958 & 2.961944 & -0.43868 \\
\hline $\mathrm{O}$ & -0.660176 & 0.425284 & 0.687227 & $\mathrm{O}$ & -0.861643 & 0.528304 & 0.083495 \\
\hline
\end{tabular}




\begin{tabular}{|c|c|c|c|c|c|c|c|}
\hline $\mathrm{O}$ & -5.178753 & 1.048985 & -0.596587 & $\mathrm{O}$ & -5.576419 & 0.985271 & -0.218979 \\
\hline $\mathrm{O}$ & 5.701229 & 1.430093 & -1.091924 & $\mathrm{O}$ & 4.848184 & 3.003525 & 0.318406 \\
\hline $\mathrm{H}$ & 2.785161 & -1.523011 & -2.125613 & $\mathrm{H}$ & 3.515395 & -0.914384 & -2.237545 \\
\hline $\mathrm{H}$ & 4.518705 & -0.728334 & 0.264809 & $\mathrm{H}$ & 4.695313 & -1.15051 & 0.574861 \\
\hline $\mathrm{H}$ & -0.957851 & -2.937934 & 1.366618 & $\mathrm{H}$ & -0.868342 & -2.674246 & 1.409753 \\
\hline $\mathrm{H}$ & -0.777022 & -3.214389 & -0.345242 & $\mathrm{H}$ & -1.147999 & -3.214698 & -0.225235 \\
\hline $\mathrm{H}$ & 2.402785 & -4.598683 & 0.227506 & $\mathrm{H}$ & 1.982019 & -4.232345 & -1.110762 \\
\hline $\mathrm{H}$ & 1.487215 & -3.703951 & -0.968759 & $\mathrm{H}$ & 1.641525 & -2.762217 & -2.000963 \\
\hline $\mathrm{H}$ & 1.314493 & 0.532807 & -1.06796 & $\mathrm{H}$ & 2.752545 & 1.984096 & 1.09856 \\
\hline $\mathrm{H}$ & 1.720323 & 2.106449 & -0.456016 & $\mathrm{H}$ & 2.883263 & 0.298255 & 1.552458 \\
\hline $\mathrm{H}$ & 1.261508 & -3.278956 & 1.747149 & $\mathrm{H}$ & 1.280666 & -3.288202 & 0.88043 \\
\hline $\mathrm{H}$ & 2.501553 & -2.103058 & 1.379878 & $\mathrm{H}$ & 2.323036 & -1.910227 & 0.662502 \\
\hline $\mathrm{H}$ & 2.535388 & 0.043543 & 1.505255 & $\mathrm{H}$ & 1.035019 & 1.647029 & -0.352832 \\
\hline $\mathrm{H}$ & 1.490221 & 1.423146 & 1.721982 & $\mathrm{H}$ & 1.751214 & 0.229197 & -1.073315 \\
\hline $\mathrm{H}$ & 3.737603 & -3.728633 & -1.702526 & $\mathrm{H}$ & 3.943367 & -3.325078 & -2.290514 \\
\hline $\mathrm{H}$ & 3.356042 & 0.917649 & -2.002937 & $\mathrm{H}$ & 3.85822 & 1.249667 & -1.172225 \\
\hline $\mathrm{H}$ & 0.806818 & -1.422807 & -0.597176 & $\mathrm{H}$ & 0.206857 & -1.442879 & -1.165172 \\
\hline $\mathrm{H}$ & 5.701965 & -2.999836 & -0.307333 & $\mathrm{H}$ & 4.251216 & -3.363256 & 0.767763 \\
\hline $\mathrm{H}$ & 4.867966 & -4.428115 & 0.335773 & $\mathrm{H}$ & 5.591201 & -3.458666 & -0.384127 \\
\hline $\mathrm{H}$ & 4.641422 & -2.852036 & 1.100106 & $\mathrm{H}$ & 4.356968 & -4.732542 & -0.342457 \\
\hline $\mathrm{H}$ & -6.421027 & -1.019811 & 0.325768 & $\mathrm{H}$ & -5.026507 & -0.92087 & 2.338013 \\
\hline $\mathrm{H}$ & -5.167165 & -1.384817 & 1.531195 & $\mathrm{H}$ & -5.759524 & -2.390908 & 1.663969 \\
\hline $\mathrm{H}$ & -5.698542 & -2.649633 & 0.404127 & $\mathrm{H}$ & -6.529516 & -0.803136 & 1.396405 \\
\hline $\mathrm{H}$ & -5.109389 & -2.510923 & -2.077281 & $\mathrm{H}$ & -5.756145 & -2.80605 & -0.859423 \\
\hline $\mathrm{H}$ & -4.192127 & -1.130062 & -2.712141 & $\mathrm{H}$ & -5.040364 & -1.617925 & -1.967065 \\
\hline $\mathrm{H}$ & -5.857922 & -0.892154 & -2.141332 & $\mathrm{H}$ & -6.538742 & -1.220335 & -1.09887 \\
\hline $\mathrm{H}$ & -0.765073 & -1.181138 & 2.856923 & $\mathrm{H}$ & -0.586895 & -0.573321 & 2.439987 \\
\hline $\mathrm{H}$ & 0.038786 & 0.354621 & 3.228499 & $\mathrm{H}$ & 0.447816 & 0.866143 & 2.422229 \\
\hline $\mathrm{H}$ & 0.974178 & -1.150816 & 3.195191 & $\mathrm{H}$ & 1.170456 & -0.752782 & 2.467887 \\
\hline $\mathrm{H}$ & -2.129791 & 4.03929 & 1.991114 & $\mathrm{H}$ & -2.470449 & 4.662868 & 1.033104 \\
\hline $\mathrm{H}$ & -1.273057 & 2.506945 & 2.269419 & $\mathrm{H}$ & -1.112927 & 5.117971 & -0.013497 \\
\hline $\mathrm{H}$ & -0.365764 & 3.960222 & 1.812069 & $\mathrm{H}$ & -2.764698 & 5.078439 & -0.662274 \\
\hline $\mathrm{H}$ & -2.248019 & 5.026681 & -0.417467 & $\mathrm{H}$ & -0.359187 & 3.588082 & -1.90326 \\
\hline $\mathrm{H}$ & -1.551934 & 4.115384 & -1.767754 & $\mathrm{H}$ & -2.009228 & 3.43379 & -2.535751 \\
\hline $\mathrm{H}$ & -0.486086 & 4.865307 & -0.565941 & $\mathrm{H}$ & -1.078674 & 1.981096 & -2.104703 \\
\hline $\mathrm{H}$ & 3.770074 & 2.383075 & 1.530202 & $\mathrm{H}$ & 6.848259 & 0.421823 & -0.581239 \\
\hline $\mathrm{H}$ & 5.477574 & 2.731054 & 1.241536 & $\mathrm{H}$ & 7.325645 & 2.092241 & -0.227951 \\
\hline $\mathrm{H}$ & 4.953335 & 1.058493 & 1.480518 & $\mathrm{H}$ & 6.235244 & 1.750345 & -1.587914 \\
\hline $\mathrm{H}$ & 4.008954 & 3.19745 & -2.169206 & $\mathrm{H}$ & 4.817452 & 1.428969 & 2.489458 \\
\hline $\mathrm{H}$ & 3.210288 & 3.688506 & -0.658824 & $\mathrm{H}$ & 5.97869 & 0.22038 & 1.897886 \\
\hline $\mathrm{H}$ & 4.937173 & 3.981269 & -0.87511 & $\mathrm{H}$ & 6.49325 & 1.890839 & 2.145956 \\
\hline $\mathrm{H}$ & -0.619027 & 2.436845 & -0.141705 & $\mathrm{H}$ & -1.103198 & 2.676162 & 0.294013 \\
\hline
\end{tabular}




\begin{tabular}{|c|c|c|c|c|c|c|c|}
\hline $\mathrm{H}$ & -4.849499 & 2.017787 & -0.466694 & $\mathrm{H}$ & -5.251693 & 1.95662 & -0.350112 \\
\hline $\mathrm{H}$ & 6.395074 & 2.067588 & -0.854931 & $\mathrm{H}$ & 5.571177 & 3.543545 & 0.677631 \\
\hline \multicolumn{4}{|c|}{ 7A (3) } & & & & \\
\hline C & -3.91846 & -1.309337 & 1.418527 & & & & \\
\hline $\mathrm{C}$ & -4.30849 & -0.60993 & 0.347507 & & & & \\
\hline $\mathrm{C}$ & 3.205563 & 0.843169 & 0.082644 & & & & \\
\hline $\mathrm{C}$ & 2.19944 & -1.44307 & -0.178514 & & & & \\
\hline $\mathrm{C}$ & 2.067081 & -0.0802 & -0.05964 & & & & \\
\hline $\mathrm{C}$ & 4.47918 & 0.298405 & -0.099024 & & & & \\
\hline $\mathrm{C}$ & 3.499848 & -2.055903 & -0.257091 & & & & \\
\hline $\mathrm{C}$ & 3.121971 & 2.268125 & 0.425097 & & & & \\
\hline $\mathrm{C}$ & 0.989199 & -2.336686 & -0.237765 & & & & \\
\hline $\mathrm{C}$ & -2.169936 & -3.062655 & 1.30421 & & & & \\
\hline $\mathrm{C}$ & -2.773681 & 0.948907 & -0.735559 & & & & \\
\hline $\mathrm{C}$ & -1.548506 & -2.505217 & -0.008023 & & & & \\
\hline $\mathrm{C}$ & -1.491958 & 0.683087 & 0.098874 & & & & \\
\hline $\mathrm{C}$ & -3.704074 & -2.804287 & 1.463068 & & & & \\
\hline $\mathrm{C}$ & -4.073245 & 0.863762 & 0.125837 & & & & \\
\hline $\mathrm{C}$ & -0.290979 & -1.609545 & 0.191221 & & & & \\
\hline $\mathrm{C}$ & 4.757252 & -1.171007 & -0.302353 & & & & \\
\hline $\mathrm{C}$ & -0.362372 & -0.217319 & -0.488952 & & & & \\
\hline $\mathrm{C}$ & -4.524829 & -3.607048 & 0.444475 & & & & \\
\hline $\mathrm{C}$ & 5.409272 & -1.360609 & -1.699584 & & & & \\
\hline $\mathrm{C}$ & 5.735523 & -1.651283 & 0.799916 & & & & \\
\hline $\mathrm{C}$ & -0.342692 & -0.269444 & -2.019154 & & & & \\
\hline $\mathrm{C}$ & 1.217058 & 3.723709 & -0.274614 & & & & \\
\hline $\mathrm{C}$ & 2.192372 & 3.937753 & 2.060622 & & & & \\
\hline $\mathrm{C}$ & -5.050718 & 3.094876 & -0.677407 & & & & \\
\hline $\mathrm{C}$ & -6.557332 & 1.363564 & 0.328261 & & & & \\
\hline $\mathrm{C}$ & 1.859046 & 2.972023 & 0.911434 & & & & \\
\hline $\mathrm{C}$ & -5.295857 & 1.590277 & -0.51873 & & & & \\
\hline $\mathrm{O}$ & 3.644888 & -3.28425 & -0.338762 & & & & \\
\hline $\mathrm{O}$ & 4.158885 & 2.977814 & 0.35907 & & & & \\
\hline $\mathrm{O}$ & 0.866205 & 0.523098 & -0.087611 & & & & \\
\hline $\mathrm{O}$ & 5.567253 & 1.038052 & -0.092367 & & & & \\
\hline $\mathrm{O}$ & -5.520931 & 1.101355 & -1.858996 & & & & \\
\hline $\mathrm{H}$ & -3.549989 & -0.766513 & 2.291933 & & & & \\
\hline $\mathrm{H}$ & -4.651278 & -1.154039 & -0.534292 & & & & \\
\hline $\mathrm{H}$ & 0.867803 & -2.753333 & -1.248595 & & & & \\
\hline $\mathrm{H}$ & 1.153468 & -3.204004 & 0.413057 & & & & \\
\hline $\mathrm{H}$ & -1.998221 & -4.144129 & 1.377194 & & & & \\
\hline $\mathrm{H}$ & -1.664189 & -2.618178 & 2.170409 & & & & \\
\hline
\end{tabular}




\begin{tabular}{|c|c|c|c|c|c|c|c|}
\hline $\mathrm{H}$ & -2.691785 & 1.934949 & -1.202438 & & & & \\
\hline $\mathrm{H}$ & -2.879633 & 0.24205 & -1.56298 & & & & \\
\hline $\mathrm{H}$ & -1.280866 & -3.338591 & -0.667765 & & & & \\
\hline $\mathrm{H}$ & -2.31257 & -1.941451 & -0.548929 & & & & \\
\hline $\mathrm{H}$ & -1.029986 & 1.651719 & 0.309754 & & & & \\
\hline $\mathrm{H}$ & -1.768236 & 0.266434 & 1.072453 & & & & \\
\hline $\mathrm{H}$ & -3.97293 & -3.166274 & 2.465855 & & & & \\
\hline $\mathrm{H}$ & -3.884637 & 1.350003 & 1.09318 & & & & \\
\hline $\mathrm{H}$ & -0.195532 & -1.384922 & 1.262165 & & & & \\
\hline $\mathrm{H}$ & -4.245148 & -3.374894 & -0.589272 & & & & \\
\hline $\mathrm{H}$ & -5.596661 & -3.405833 & 0.552644 & & & & \\
\hline $\mathrm{H}$ & -4.363444 & -4.68074 & 0.593402 & & & & \\
\hline $\mathrm{H}$ & 4.745968 & -1.010251 & -2.497918 & & & & \\
\hline $\mathrm{H}$ & 5.602985 & -2.425298 & -1.852153 & & & & \\
\hline $\mathrm{H}$ & 6.349995 & -0.807394 & -1.758966 & & & & \\
\hline $\mathrm{H}$ & 5.906629 & -2.722074 & 0.668526 & & & & \\
\hline $\mathrm{H}$ & 5.318046 & -1.487395 & 1.799451 & & & & \\
\hline $\mathrm{H}$ & 6.686596 & -1.118415 & 0.728395 & & & & \\
\hline $\mathrm{H}$ & 0.592249 & -0.705062 & -2.381789 & & & & \\
\hline $\mathrm{H}$ & -0.437841 & 0.736198 & -2.440094 & & & & \\
\hline $\mathrm{H}$ & -1.166206 & -0.879659 & -2.401181 & & & & \\
\hline $\mathrm{H}$ & 0.946972 & 3.039229 & -1.083839 & & & & \\
\hline $\mathrm{H}$ & 0.307938 & 4.236129 & 0.058921 & & & & \\
\hline $\mathrm{H}$ & 1.908199 & 4.476268 & -0.67064 & & & & \\
\hline $\mathrm{H}$ & 2.891884 & 4.713415 & 1.737716 & & & & \\
\hline $\mathrm{H}$ & 2.638586 & 3.409204 & 2.910545 & & & & \\
\hline $\mathrm{H}$ & 1.273256 & 4.421988 & 2.408294 & & & & \\
\hline $\mathrm{H}$ & -4.787115 & 3.548988 & 0.283643 & & & & \\
\hline $\mathrm{H}$ & -5.958111 & 3.580899 & -1.051172 & & & & \\
\hline $\mathrm{H}$ & -4.246427 & 3.300243 & -1.389196 & & & & \\
\hline $\mathrm{H}$ & -6.783133 & 0.295341 & 0.425654 & & & & \\
\hline $\mathrm{H}$ & -6.431818 & 1.767572 & 1.339295 & & & & \\
\hline $\mathrm{H}$ & -7.41595 & 1.858814 & -0.137688 & & & & \\
\hline $\mathrm{H}$ & 1.151947 & 2.225897 & 1.270946 & & & & \\
\hline $\mathrm{H}$ & 5.239003 & 2.001307 & 0.051243 & & & & \\
\hline $\mathrm{H}$ & -5.918265 & 0.218562 & -1.788122 & & & & \\
\hline
\end{tabular}

Key transitions, oscillator strengths, and rotatory strengths in the ECD of conformers 7A (1) at B3LYP /6-311++G (d,p) level.

\begin{tabular}{|l|l|l|l|l|l|}
\hline Species & Excited State & $\Delta \mathrm{E}(\mathrm{eV})^{\mathrm{a}}$ & $\lambda(\mathrm{nm})^{\mathrm{b}}$ & $\mathrm{f}^{\mathrm{c}}$ & Rvel $^{\mathrm{d}}$ \\
\hline 7A $(1)$ & $121->127$ & 3.4993 & 354.31 & 0.0099 & 85.85 \\
\hline & $122->127$ & 3.9339 & 315.17 & 0.0004 & 73.63 \\
\hline
\end{tabular}




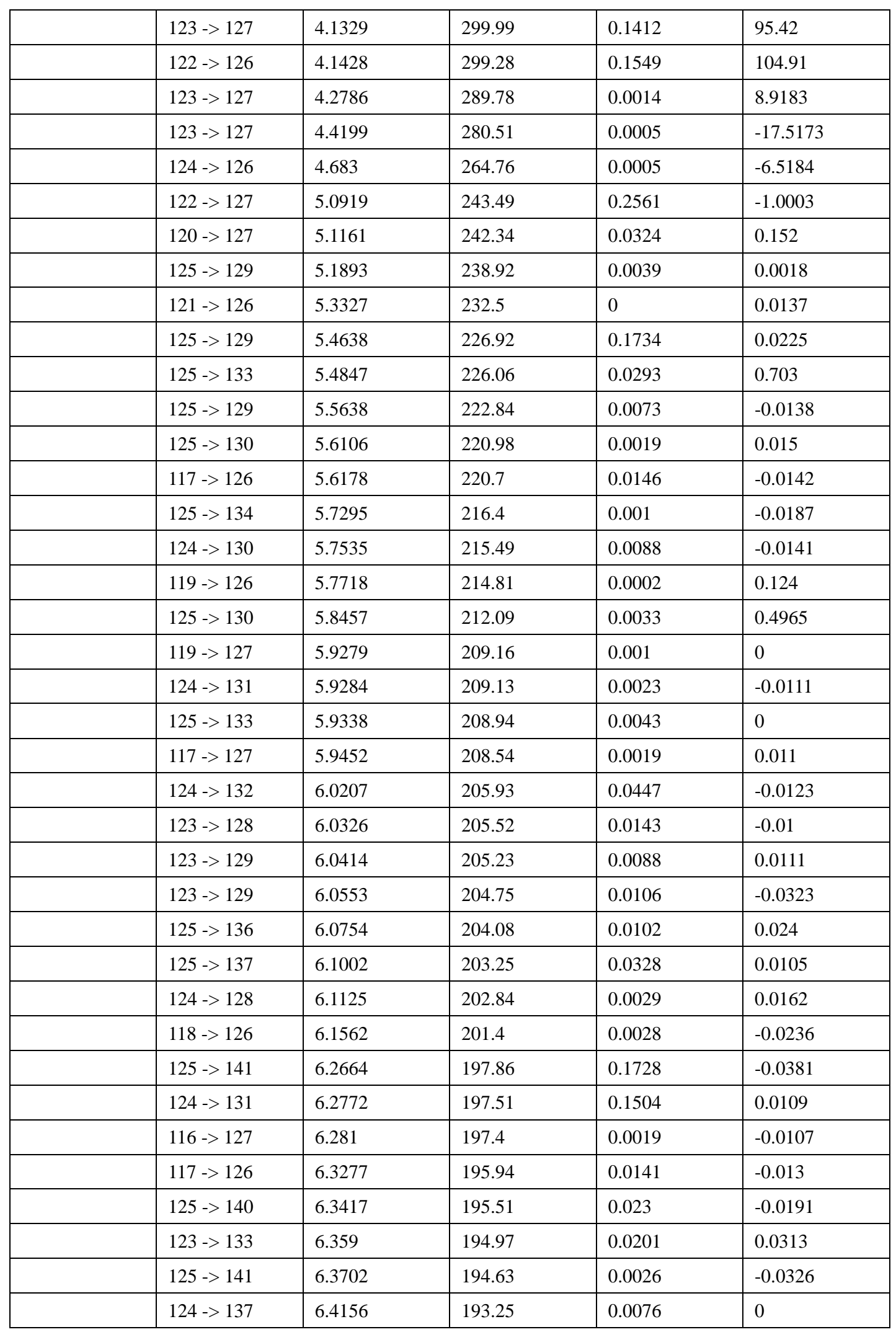

${ }^{\mathrm{a}}$ Excitation energy. ${ }^{\mathrm{b}}$ Wavelength. ${ }^{\mathrm{c}}$ Oscillator strength. ${ }^{\mathrm{d}}$ Rotatory strength in velocity form $(10-40 \mathrm{cgs}$.). 


\section{Single-crystal X-ray data for $1 \mathrm{~b}$}

Crystal data for 1b: $\mathrm{C}_{28} \mathrm{H}_{40} \mathrm{O}_{5}, M=456.60, a=8.7878(5) \AA, b=20.2223(11) \AA, c$ $=29.8190(16) \AA, \alpha=90^{\circ}, \beta=90^{\circ}, \gamma=90^{\circ}, V=5299.1(5) \AA^{3}, T=298(2) \mathrm{K}$, space group $P 212121, Z=8, \mu(\mathrm{CuK} \alpha)=0.613 \mathrm{~mm}^{-1}, 22780$ reflections measured, 6573 independent reflections $\left(R_{\text {int }}=0.0535\right)$. The final $R_{l}$ values were $0.0448(I>2 \sigma(I))$. The final $w R\left(F^{2}\right)$ values were $0.1168(I>2 \sigma(I))$. The final $R_{l}$ values were 0.0473 (all data).

The final $w R\left(F^{2}\right)$ values were 0.1191 (all data). The goodness of fit on $F^{2}$ was 1.065 . Flack parameter $=0.11(8)$. 


\section{HRESIMS of compound $\mathbf{1 a} / \mathbf{1 b}$}

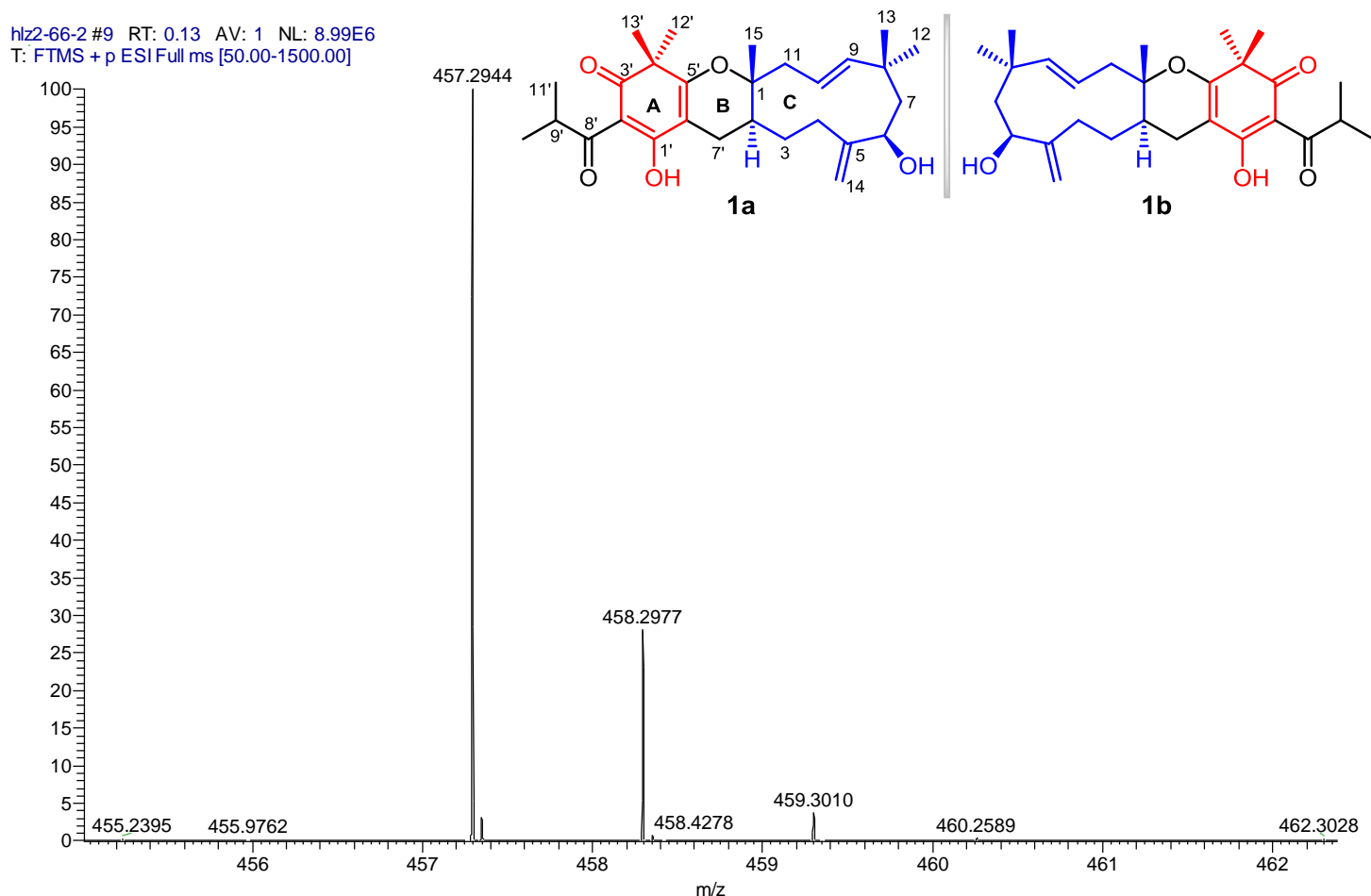




\section{${ }^{1} \mathrm{H}$ NMR of compound $\mathbf{1 a} / \mathbf{1 b}$ (in $\mathrm{CDCl}_{3}$ )}

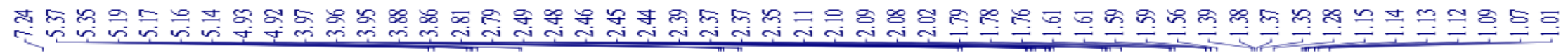
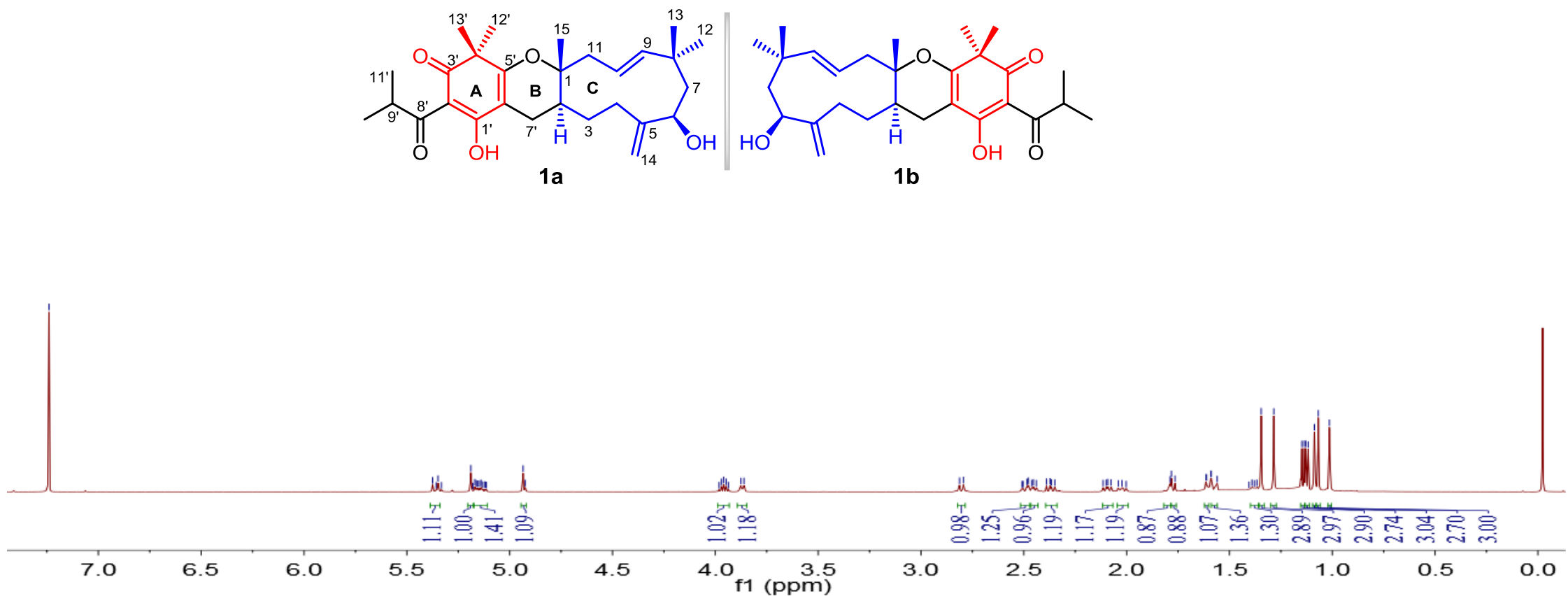
${ }^{13} \mathrm{C}$ NMR of compound $\mathbf{1 a} / \mathbf{1 b}$ (in $\mathrm{CDCl}_{3}$ )
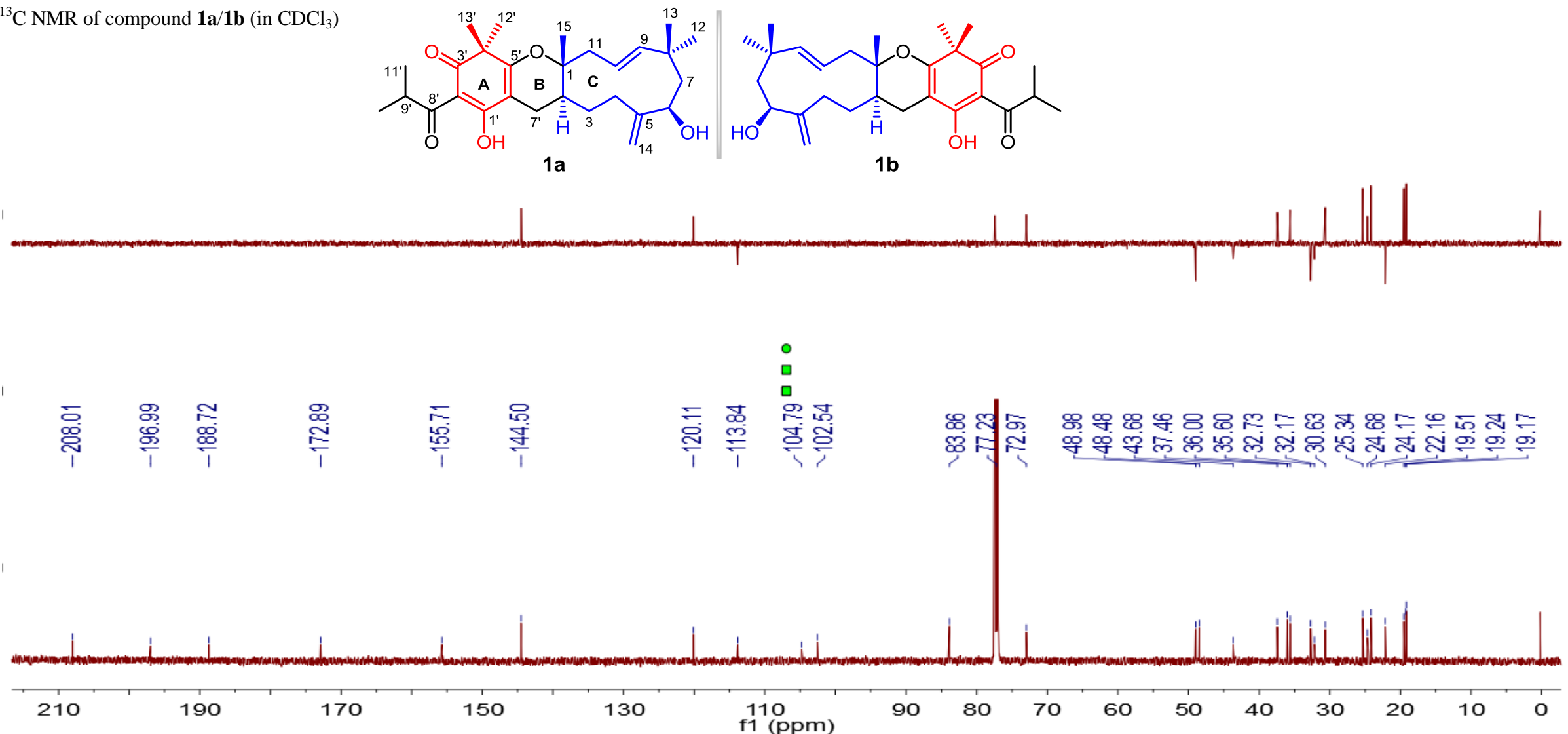


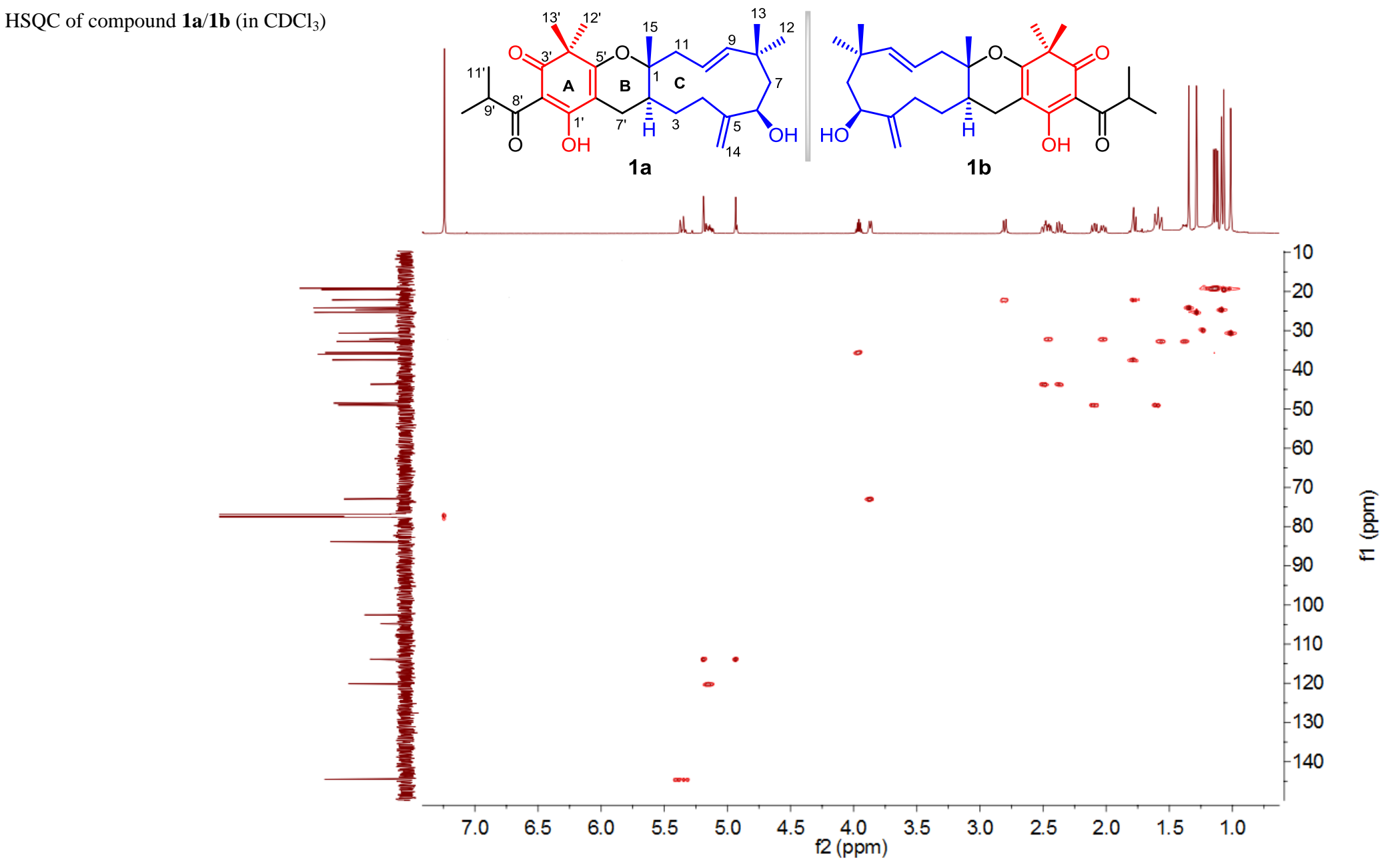


HMBC of compound $\mathbf{1 a} / \mathbf{1} \mathbf{b}$ (in $\mathrm{CDCl}_{3}$ )

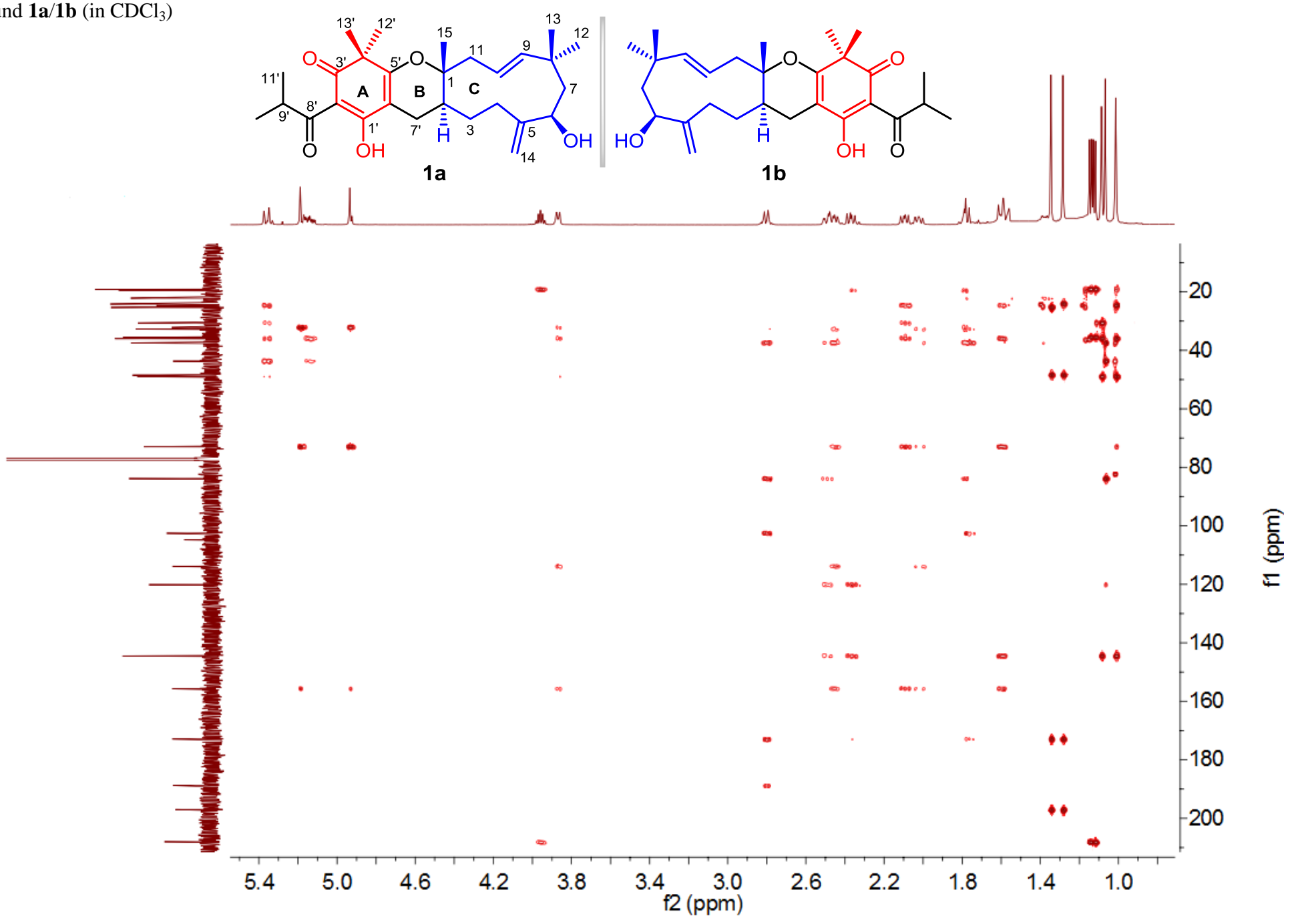




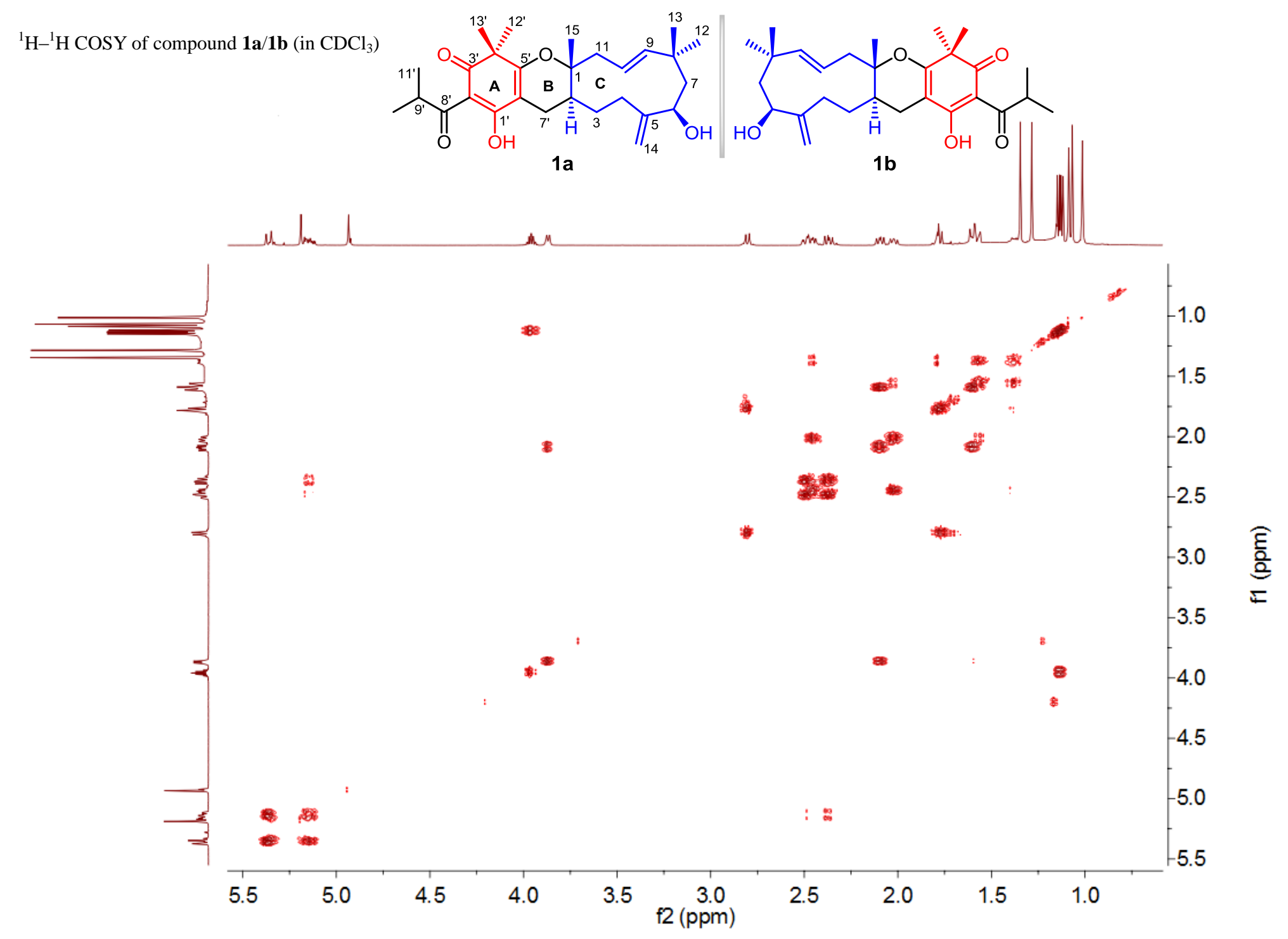




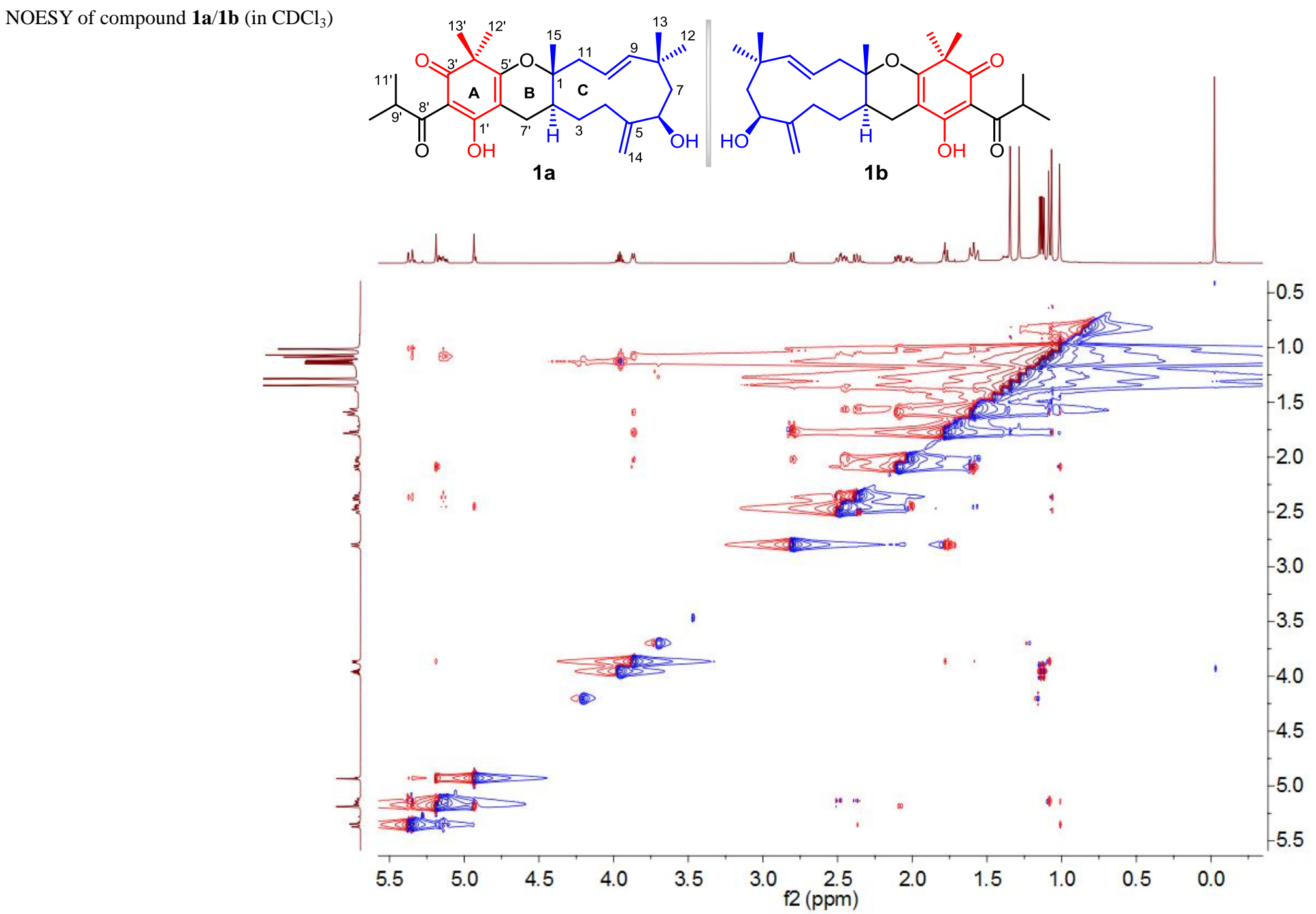




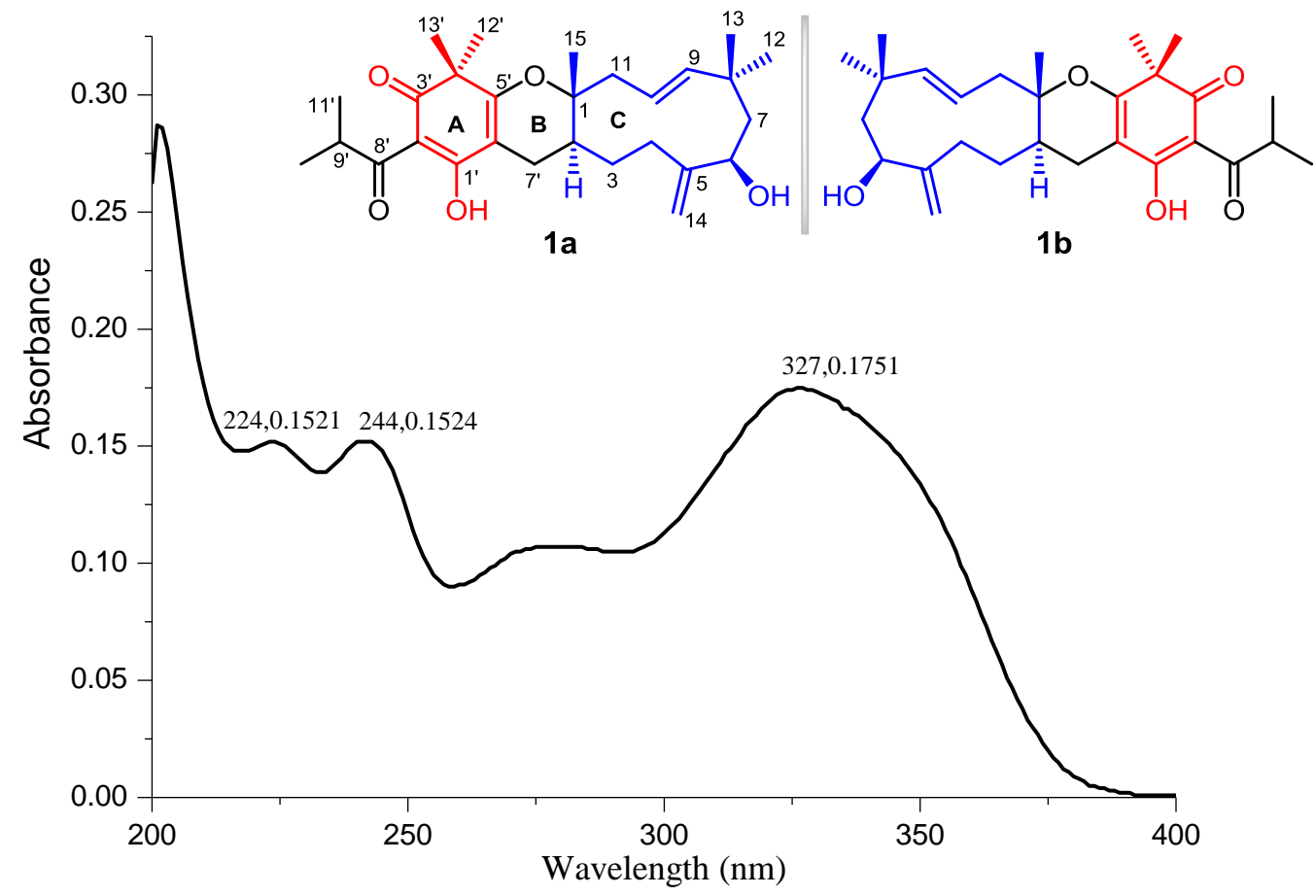


IR of compound $\mathbf{1 a} / \mathbf{1} \mathbf{b}(\mathrm{KBr}$ disc)

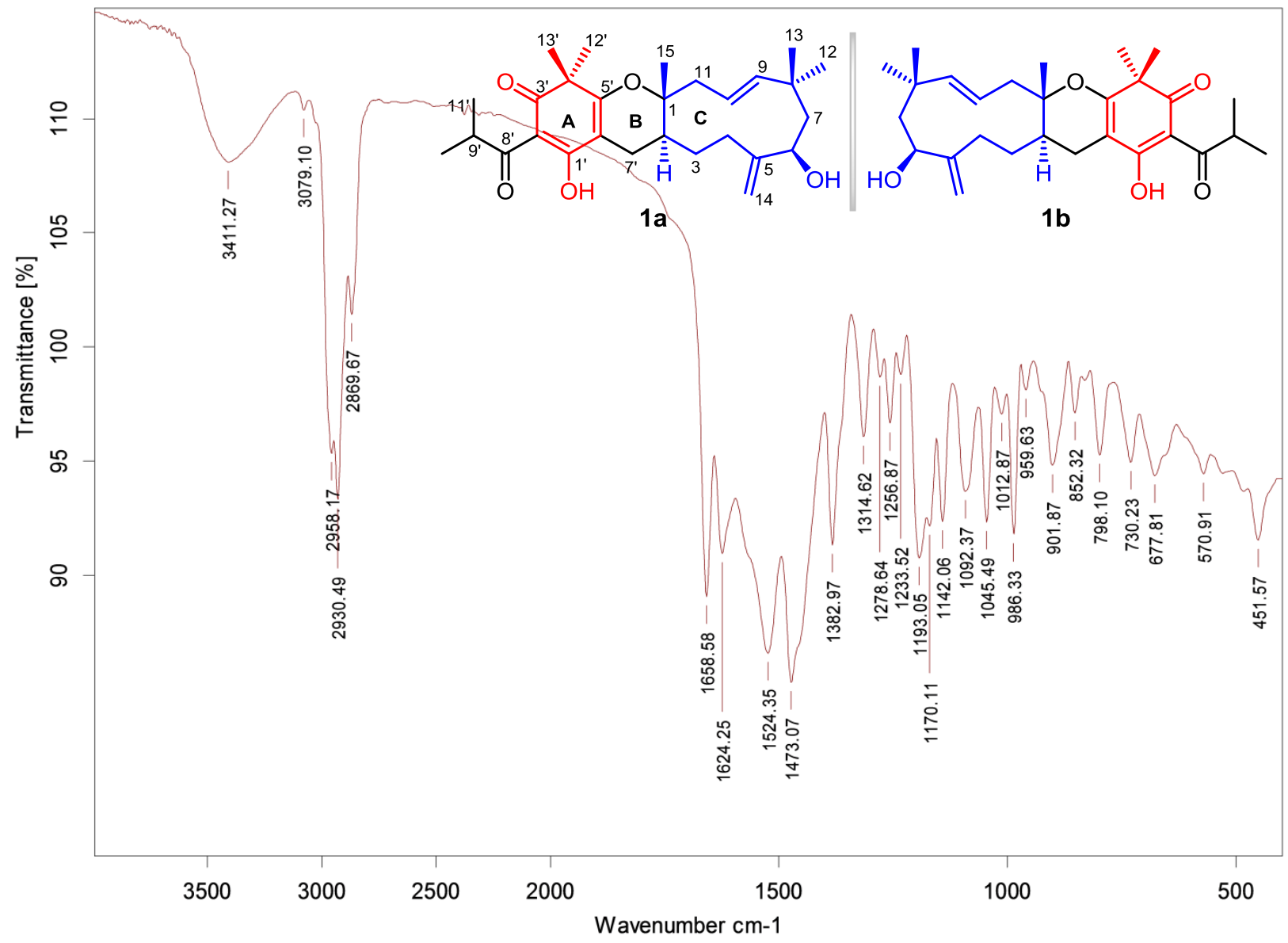


HRESIMS of compound $\mathbf{2 a / 2 b}$

hlz2-65-1 \#18 RT: $0.27 \quad$ AV: $1 \quad \mathrm{NL}: 3.44 \mathrm{E} 6$

T: FTMS + p ESI Full ms [50.00-1500.00]

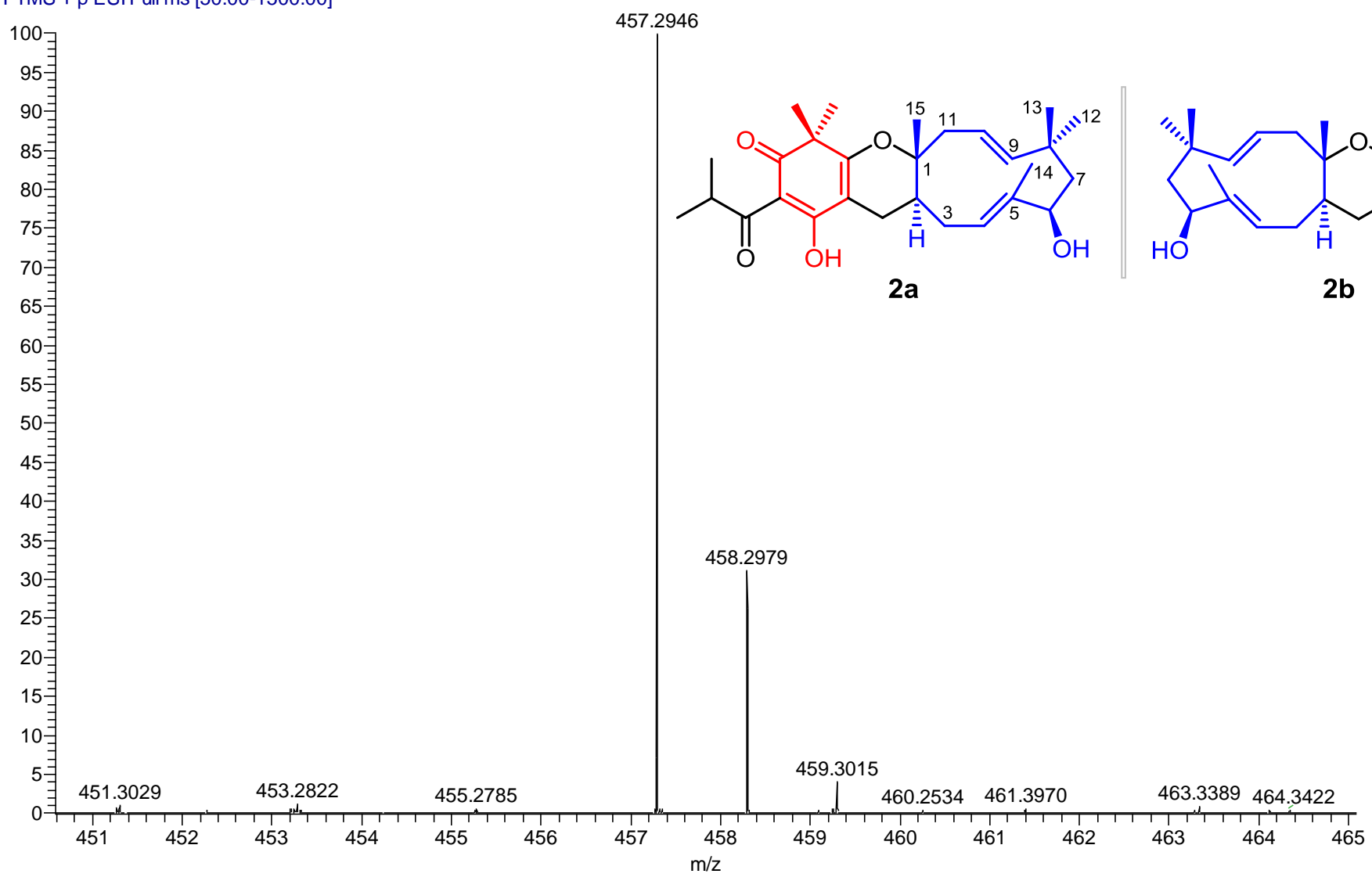




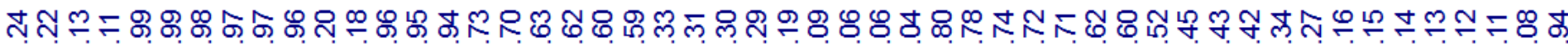

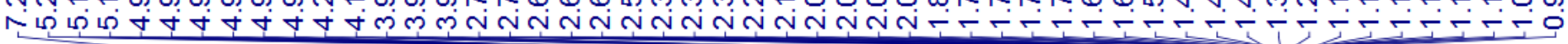

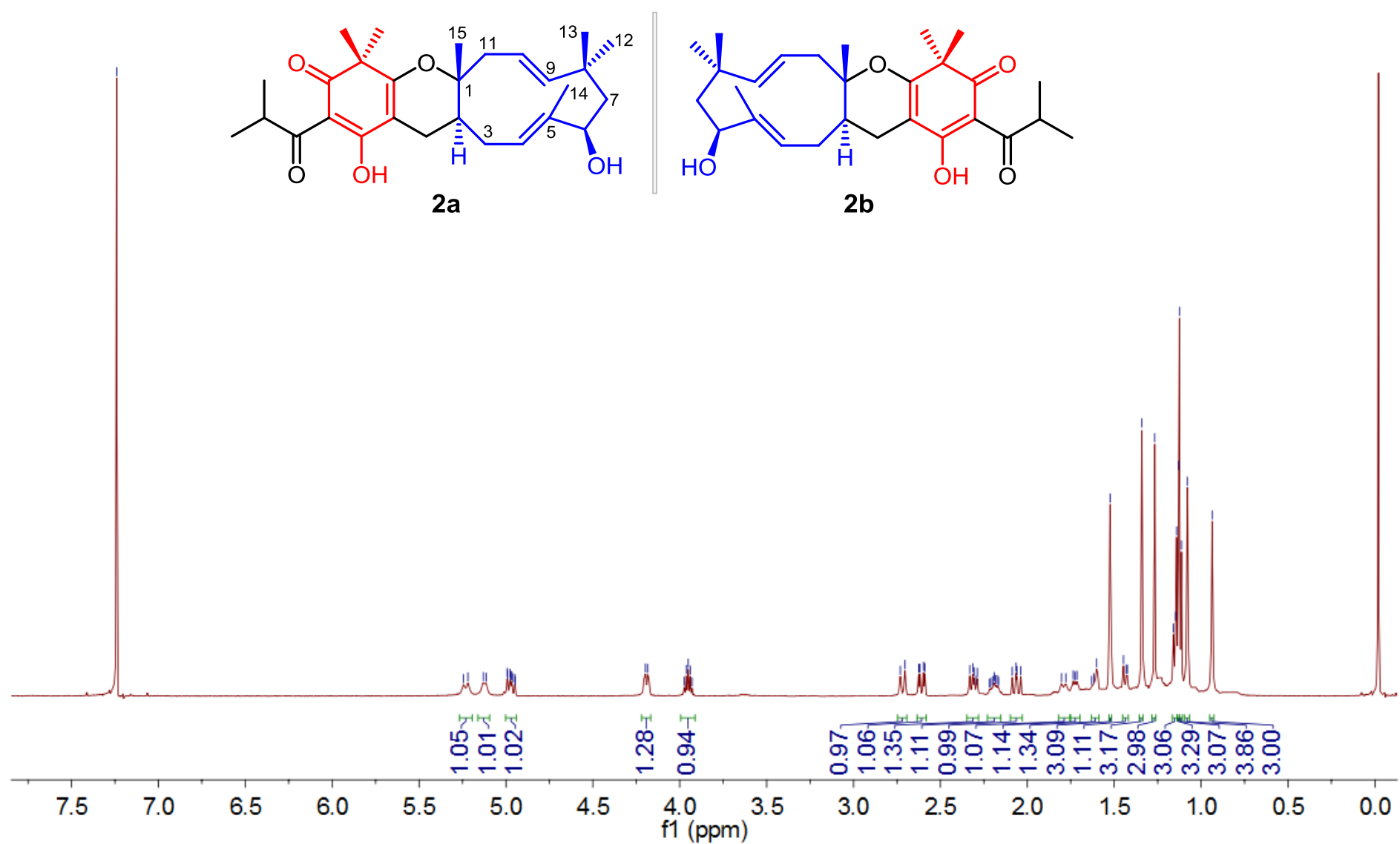


${ }^{13} \mathrm{C}$ NMR of compound $\mathbf{2 a} / \mathbf{2 b}$ (in $\mathrm{CDCl}_{3}$ )
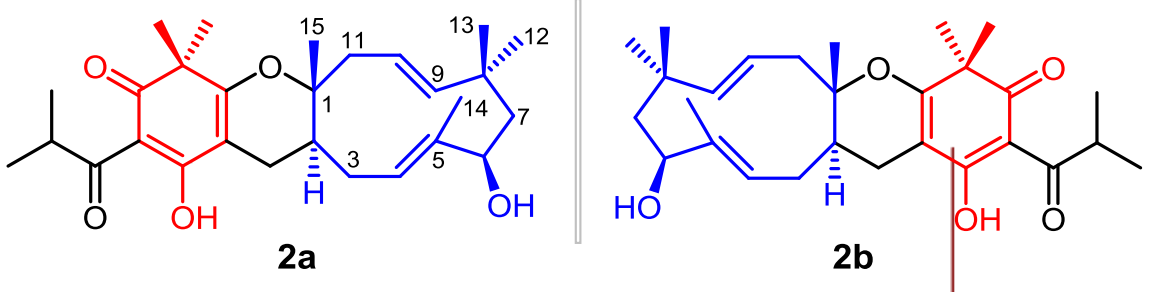

Non

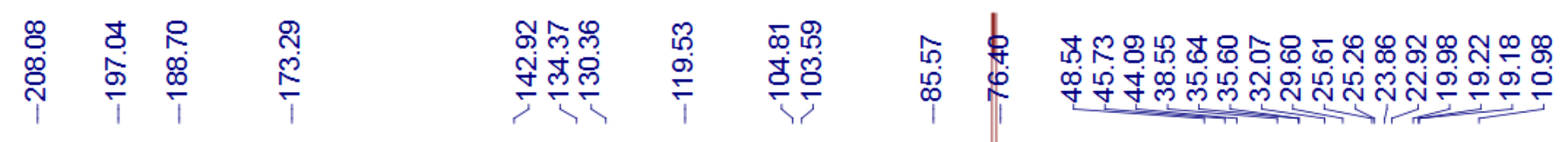

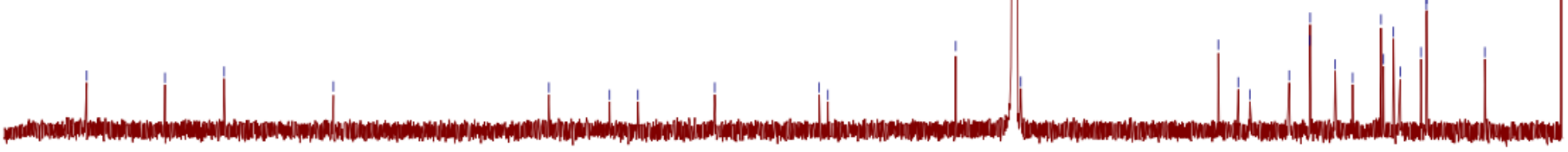

210

190

170

150

130

110
$\mathrm{f} 1(\mathrm{ppm})$

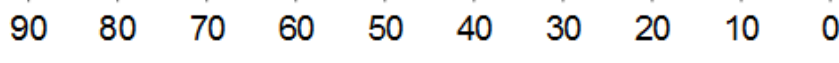




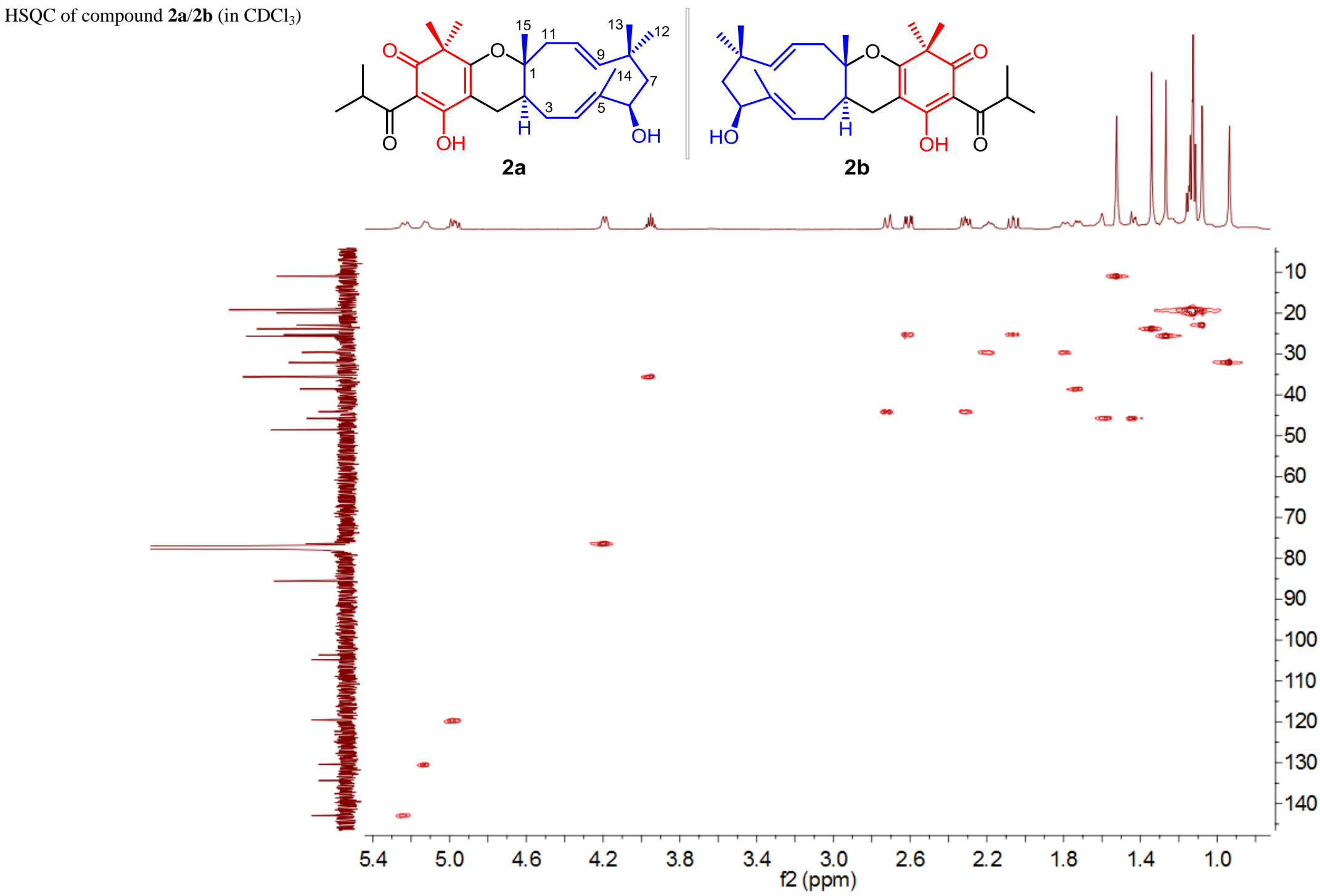




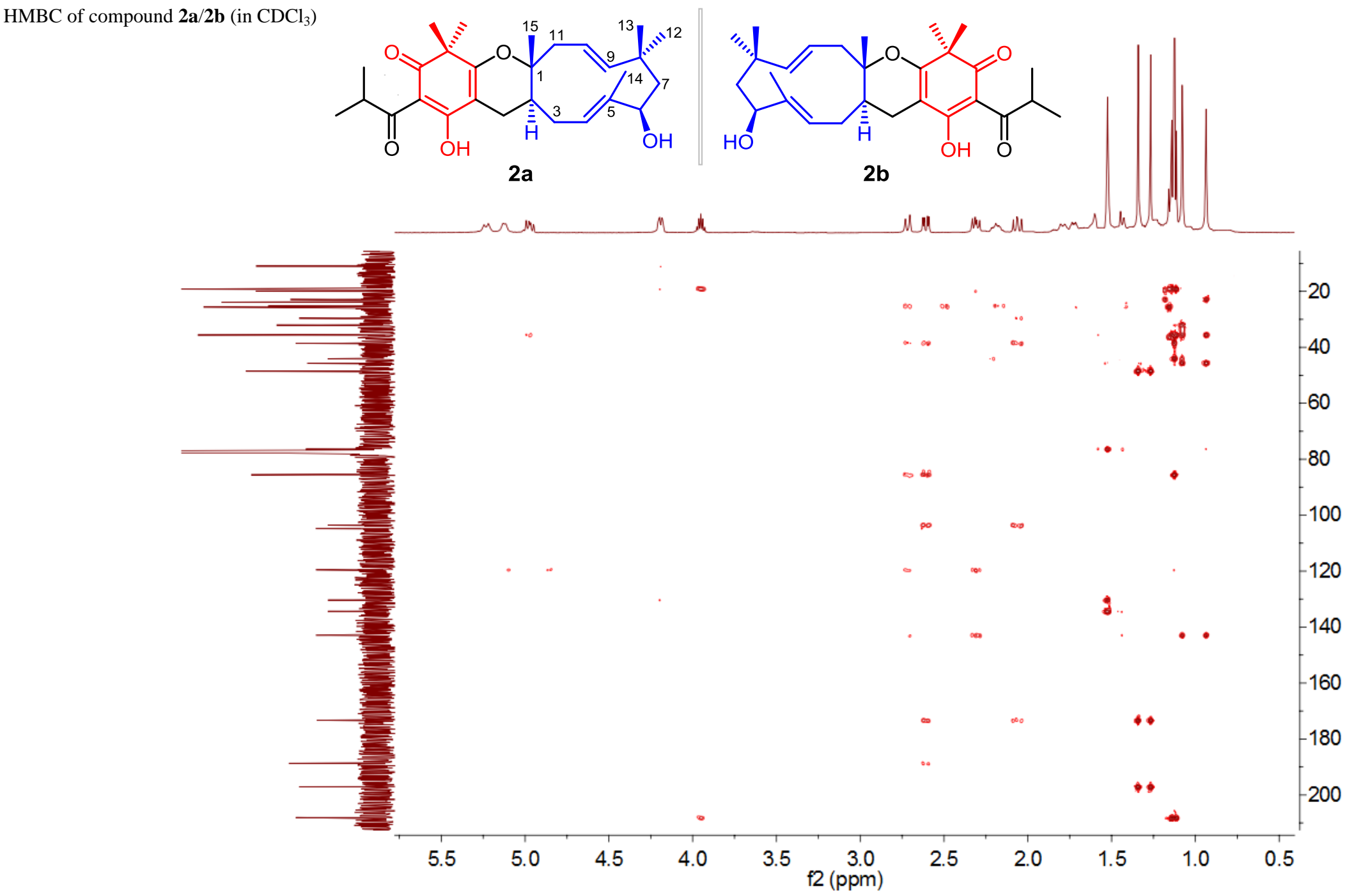




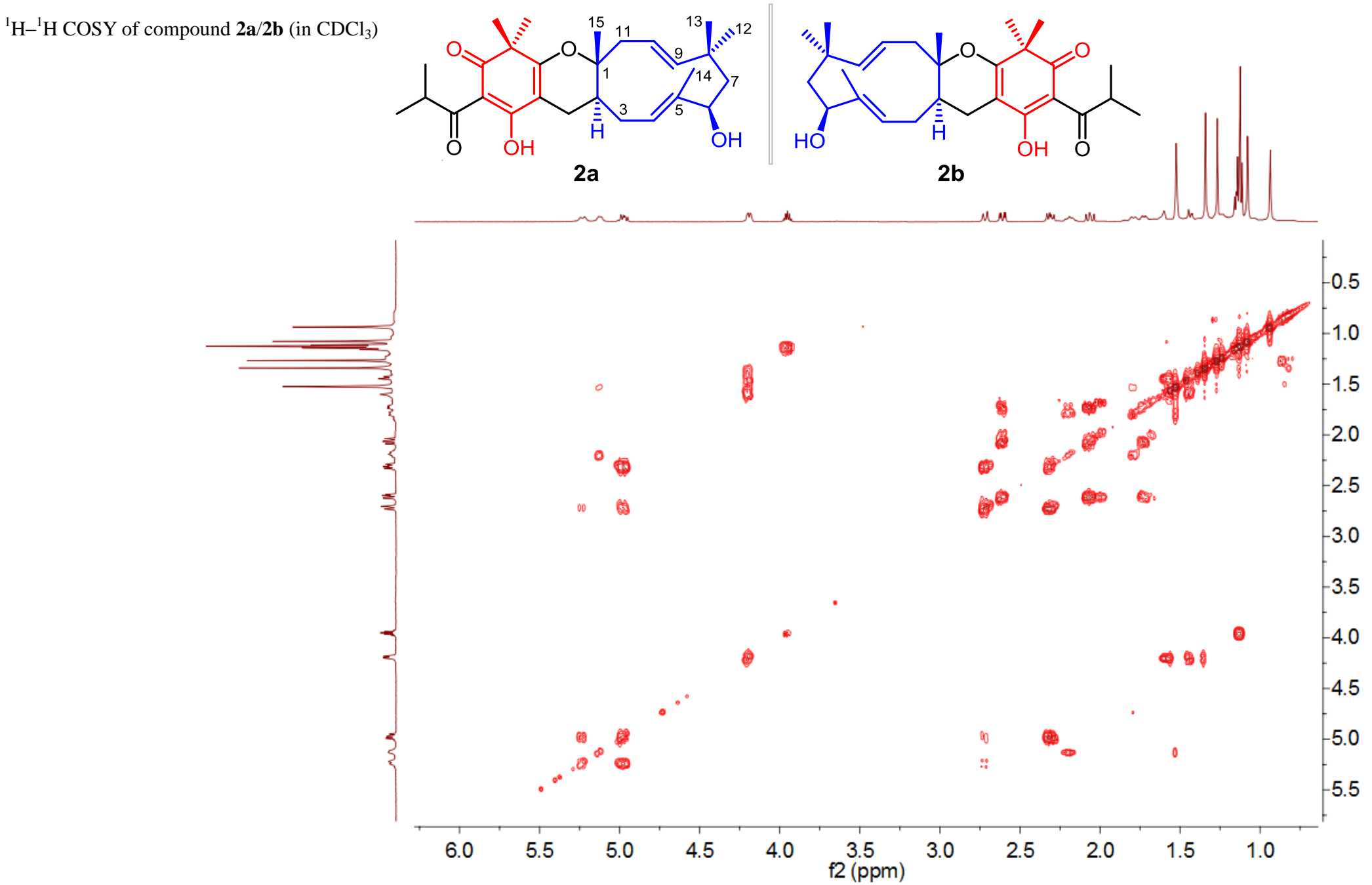




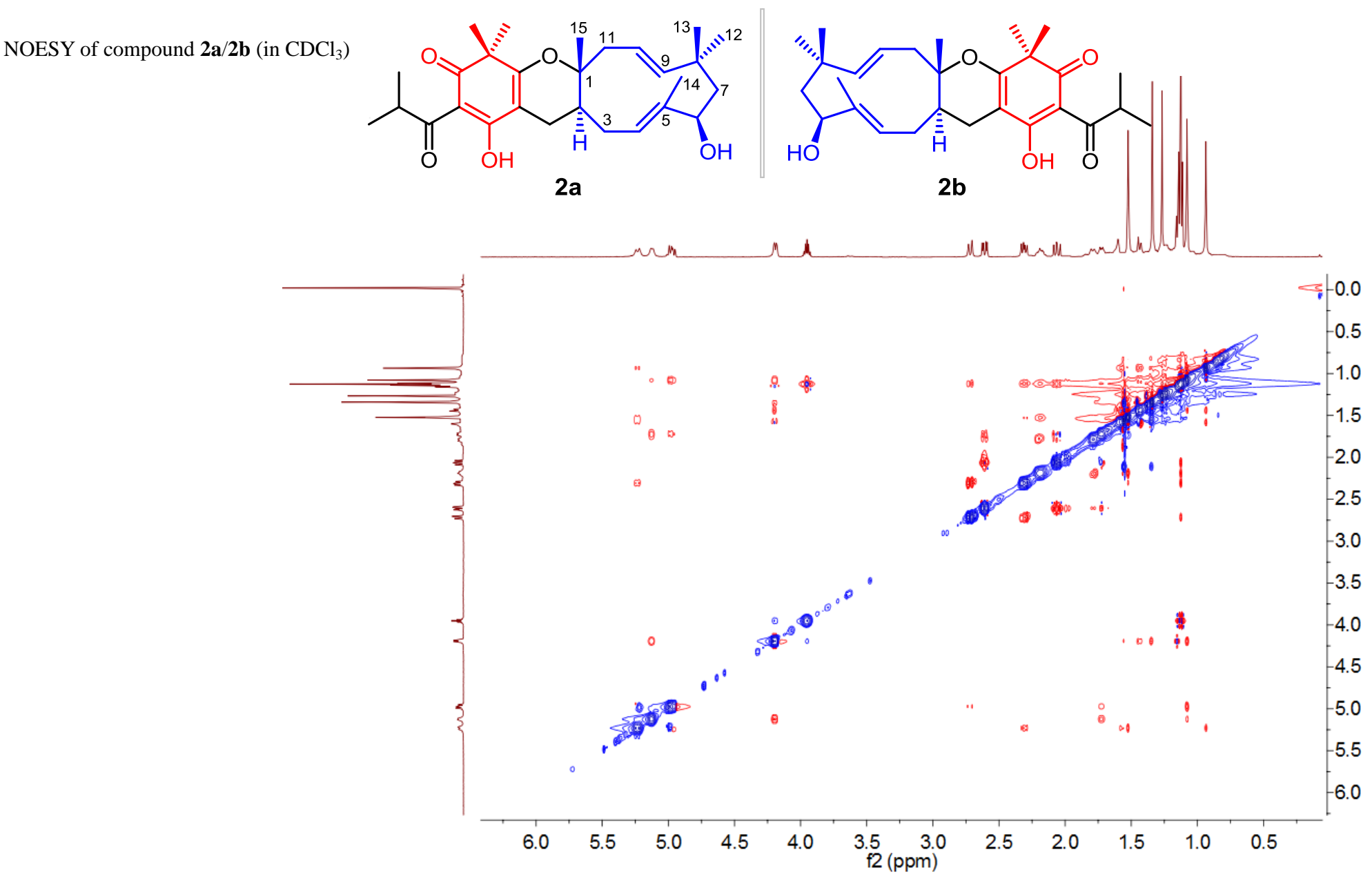


UV of compound 2 (in $\mathrm{MeOH}$ )

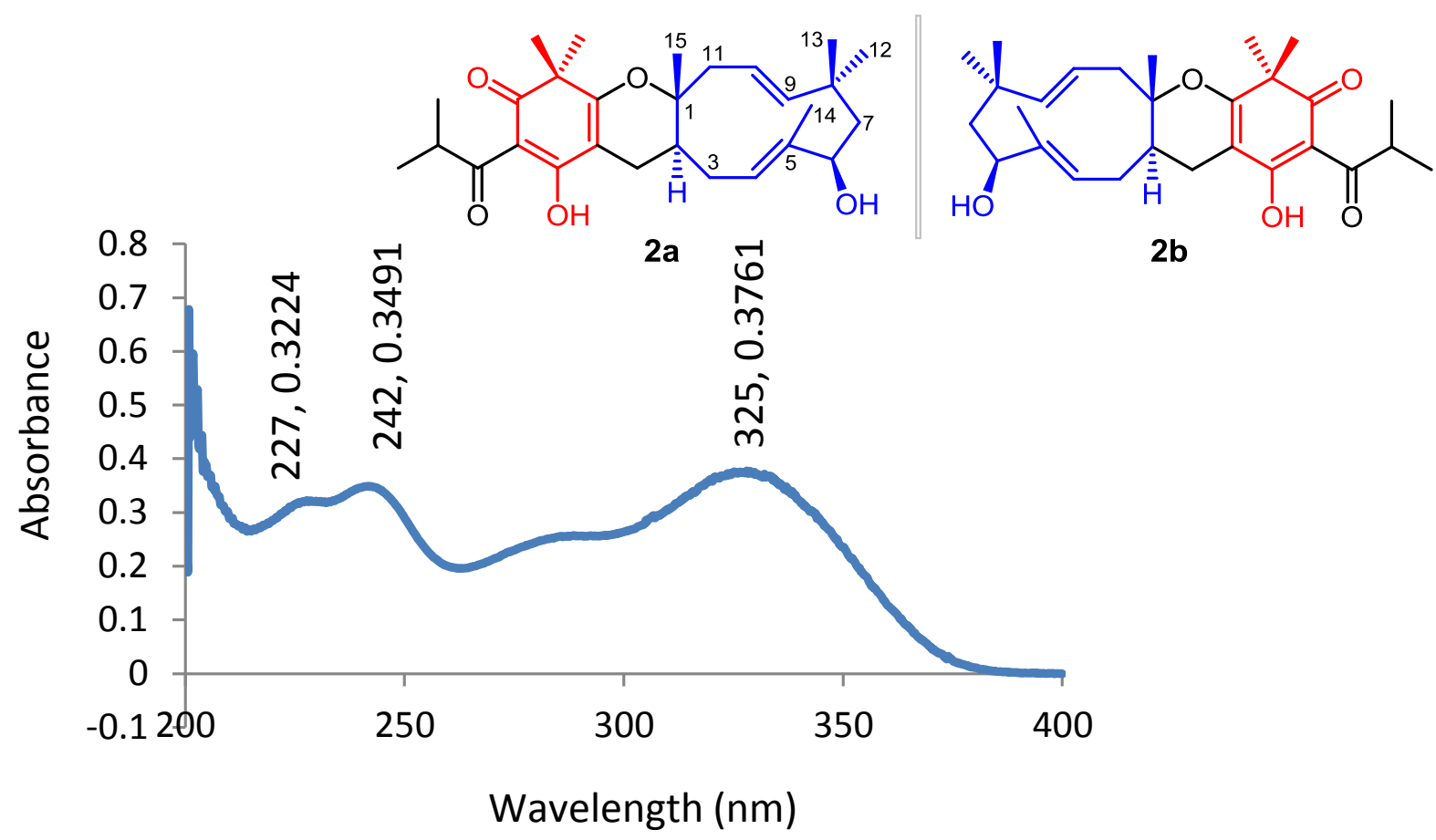


IR of compound 2 ( $\mathrm{KBr}$ disc)

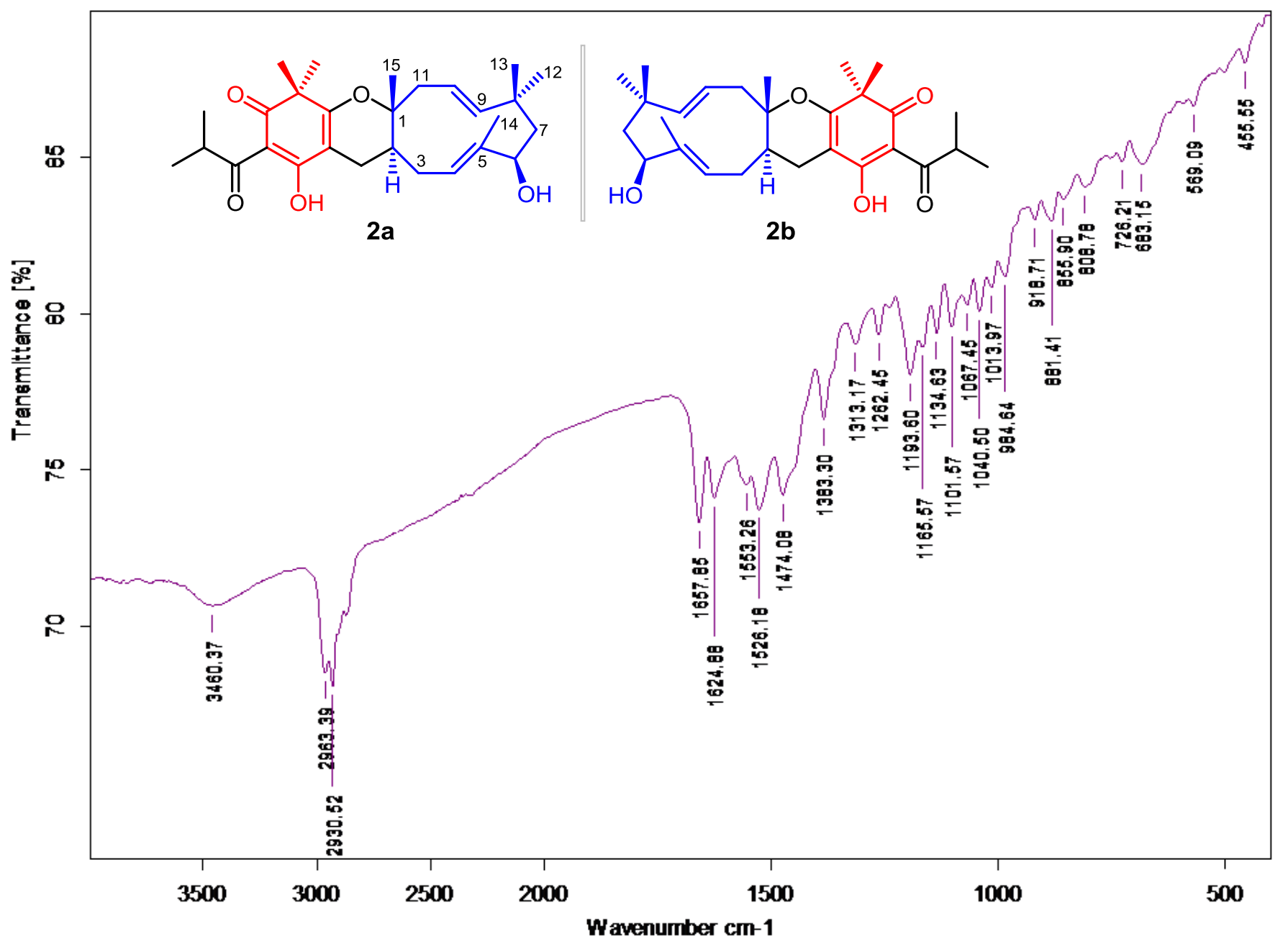


HRESIMS of compound $\mathbf{3 a} / \mathbf{3 b}$

hlzz-61-1-1 \#9-11 RT: 0.12-0.16 AV: 3 NL: 9.24E7

$\mathrm{T}: \mathrm{FTMS}+\mathrm{p}$ ESI Full ms [50.00-1500.00]

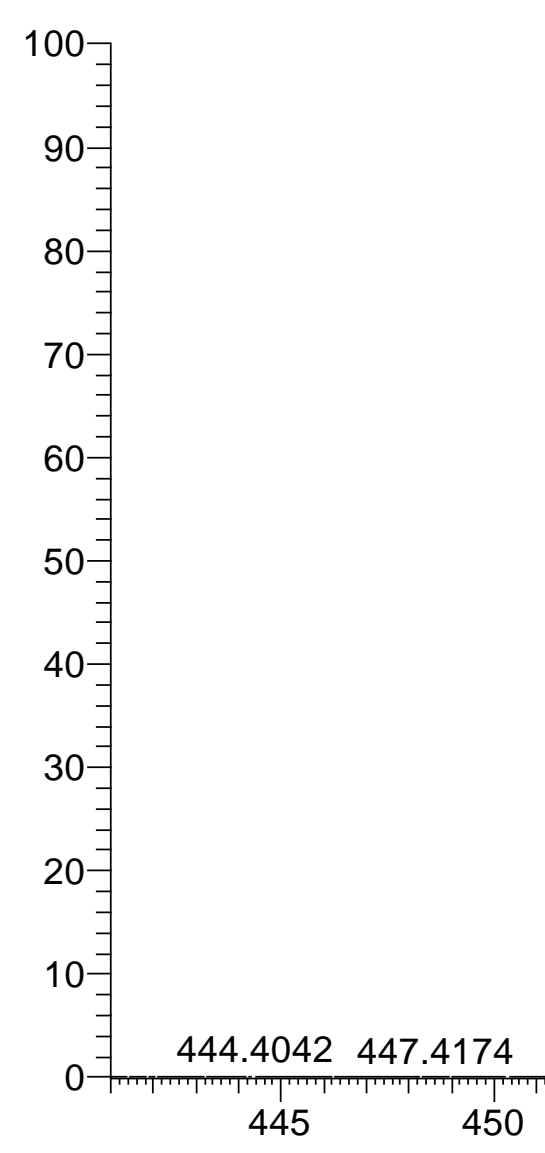

457.2941

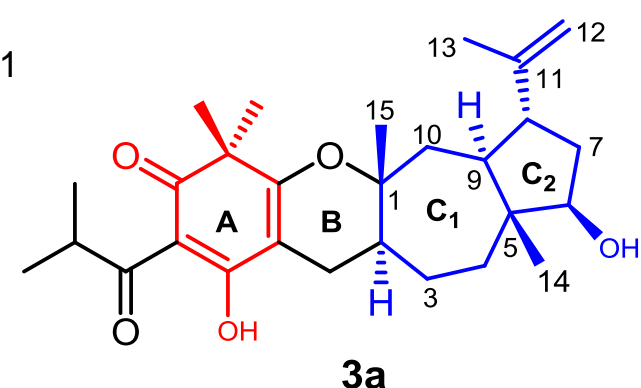

$3 a$

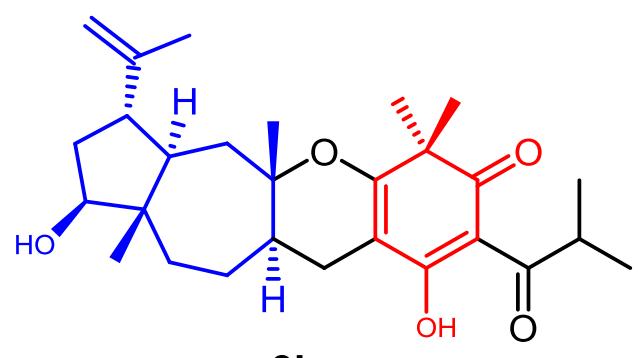

$3 b$

479.2759

458.2975

459.3008

480.2793 
${ }^{1} \mathrm{H}$ NMR of compound $\mathbf{3 a} / \mathbf{3 b}$ (in $\mathrm{CDCl}_{3}$ )

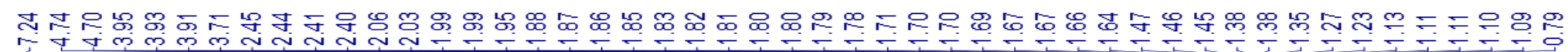

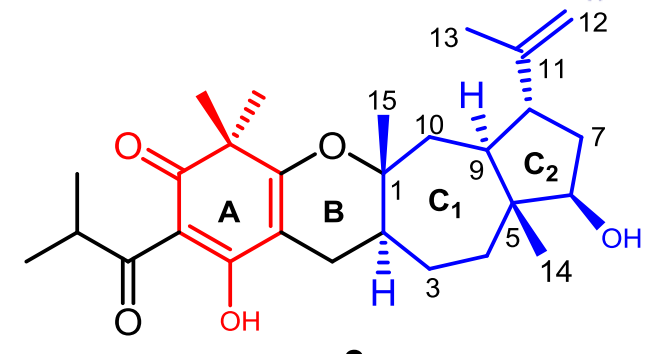

$3 a$
$3 b$

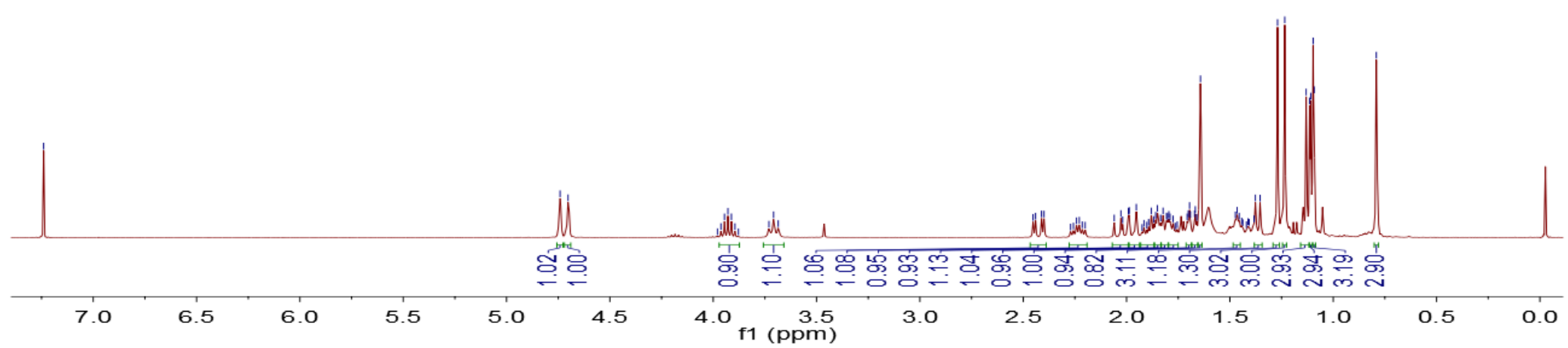


${ }^{13} \mathrm{C}$ NMR of compound $\mathbf{3 a} / \mathbf{3 b}$ (in $\mathrm{CDCl}_{3}$ )

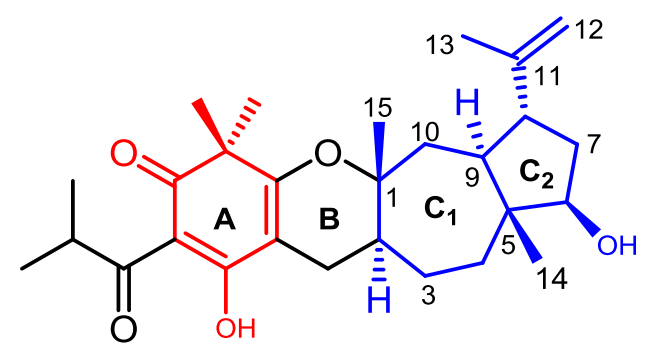

$3 a$

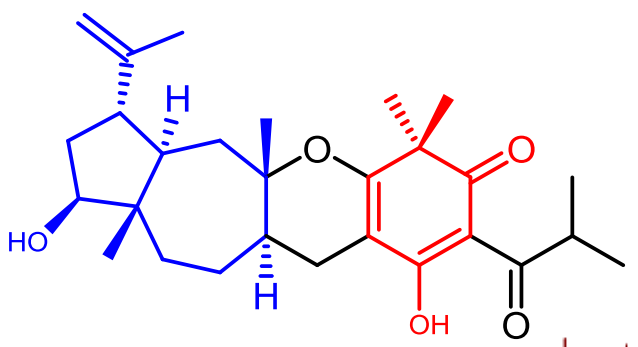

3b

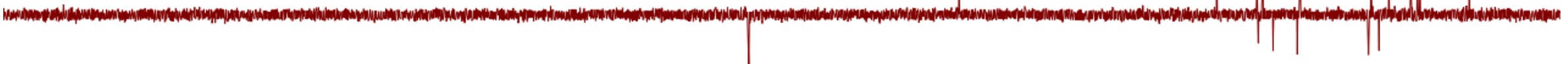

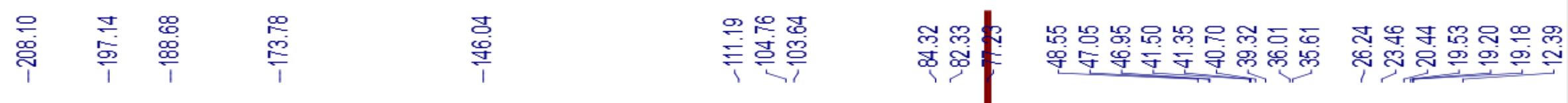

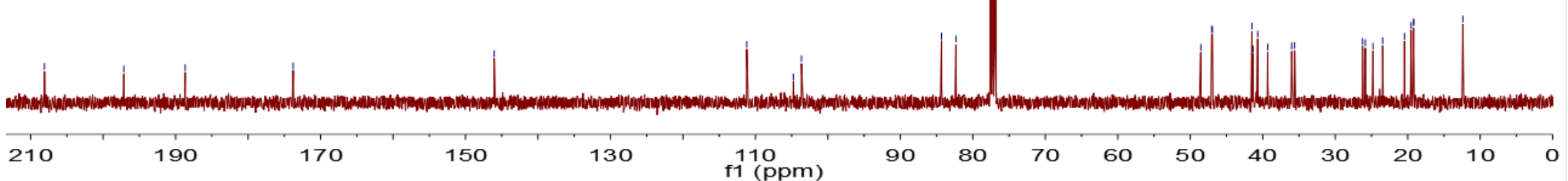




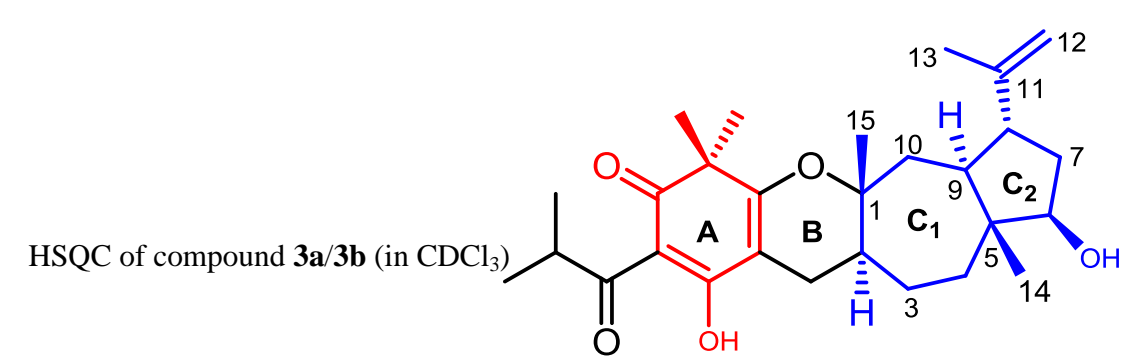

$3 a$

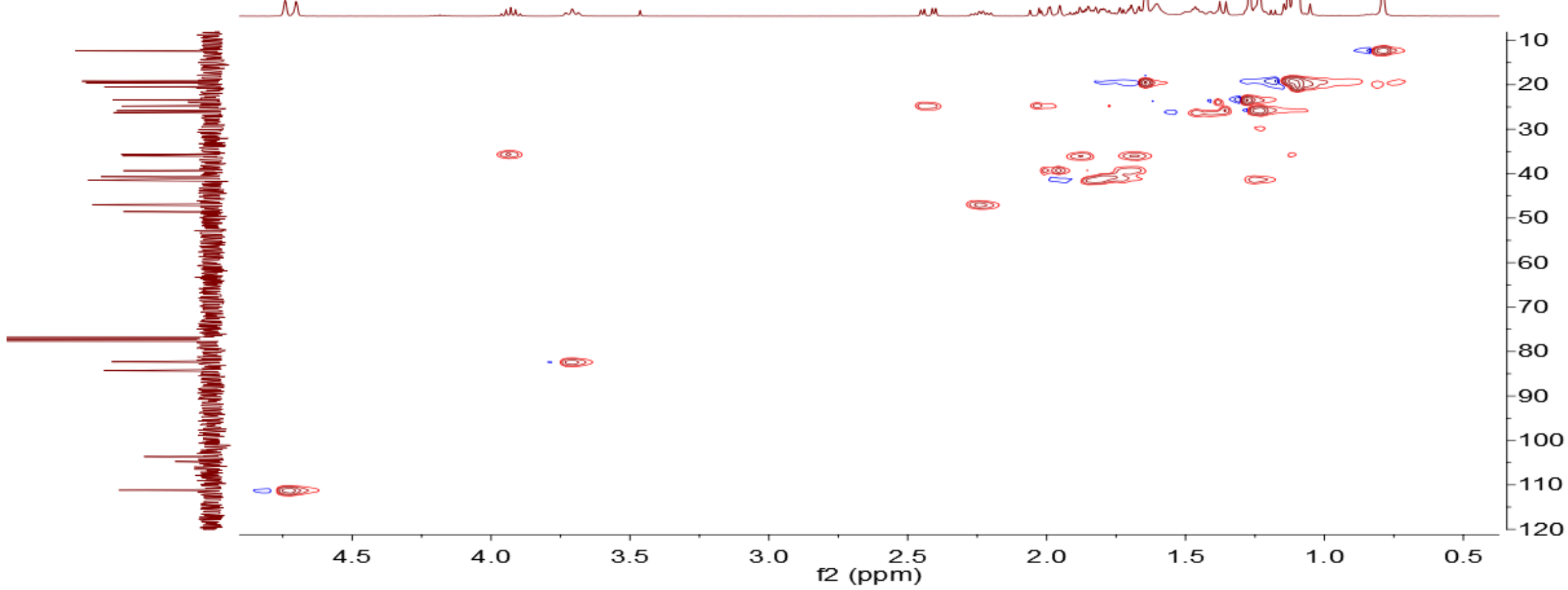




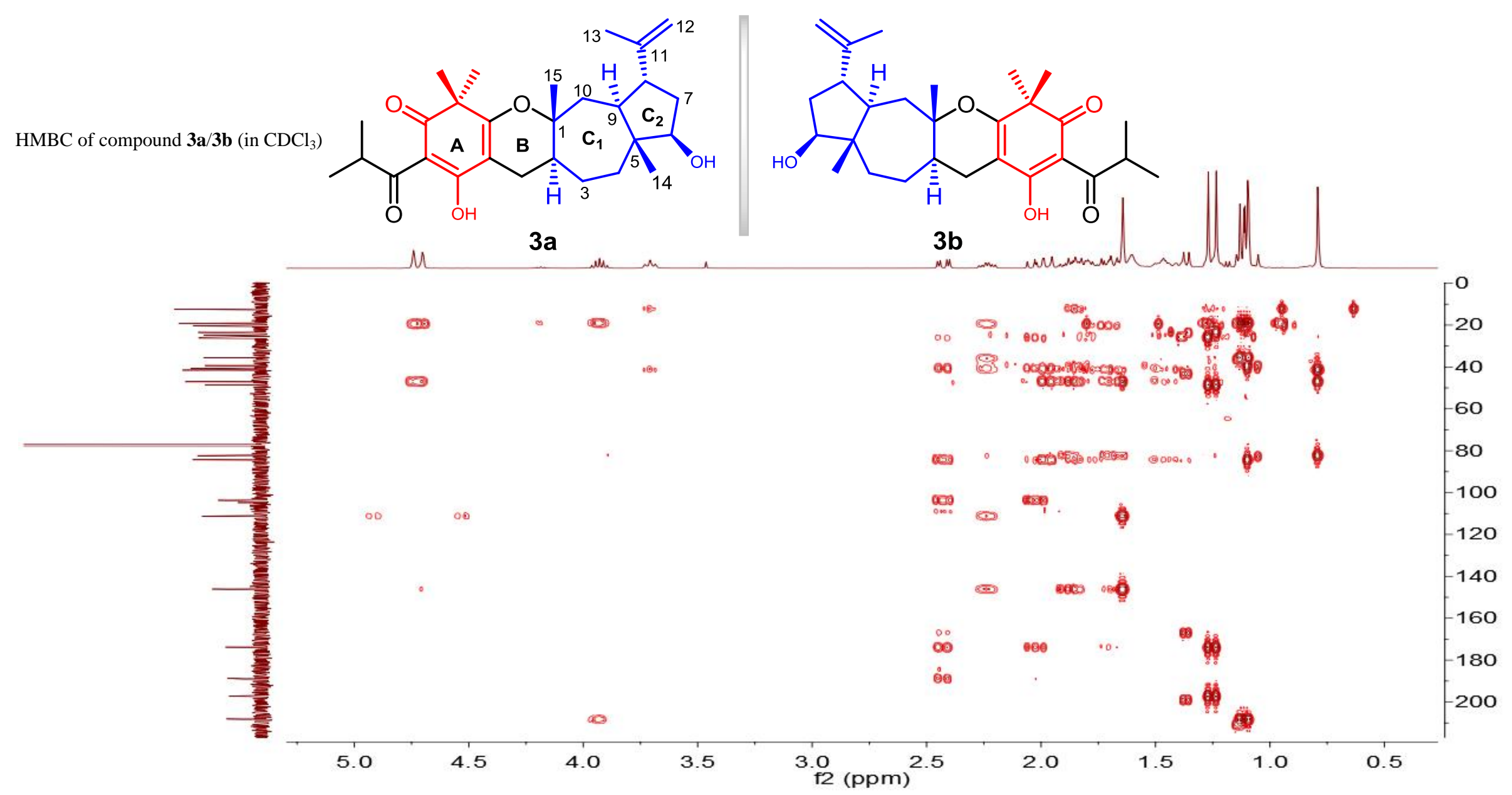




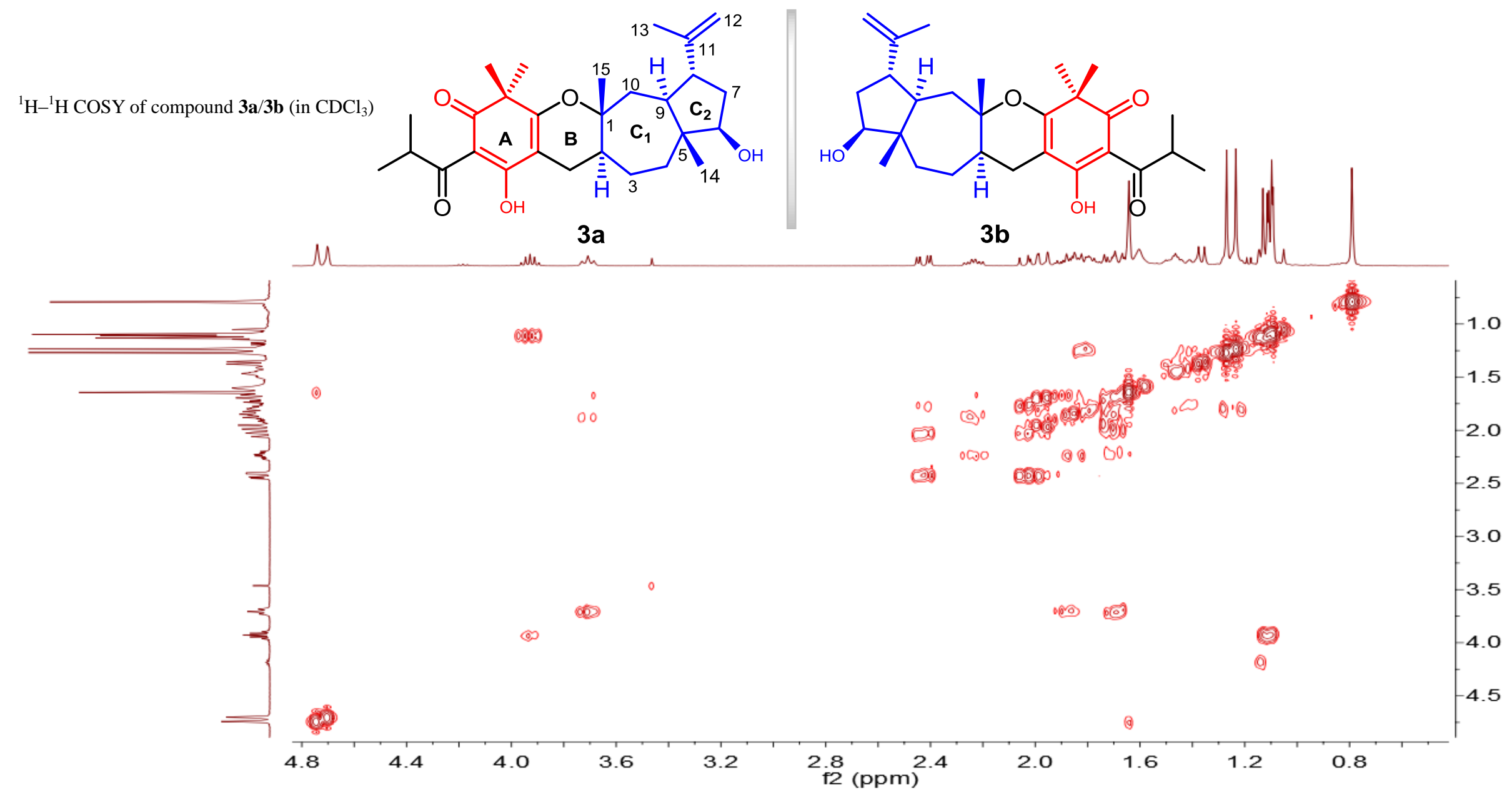




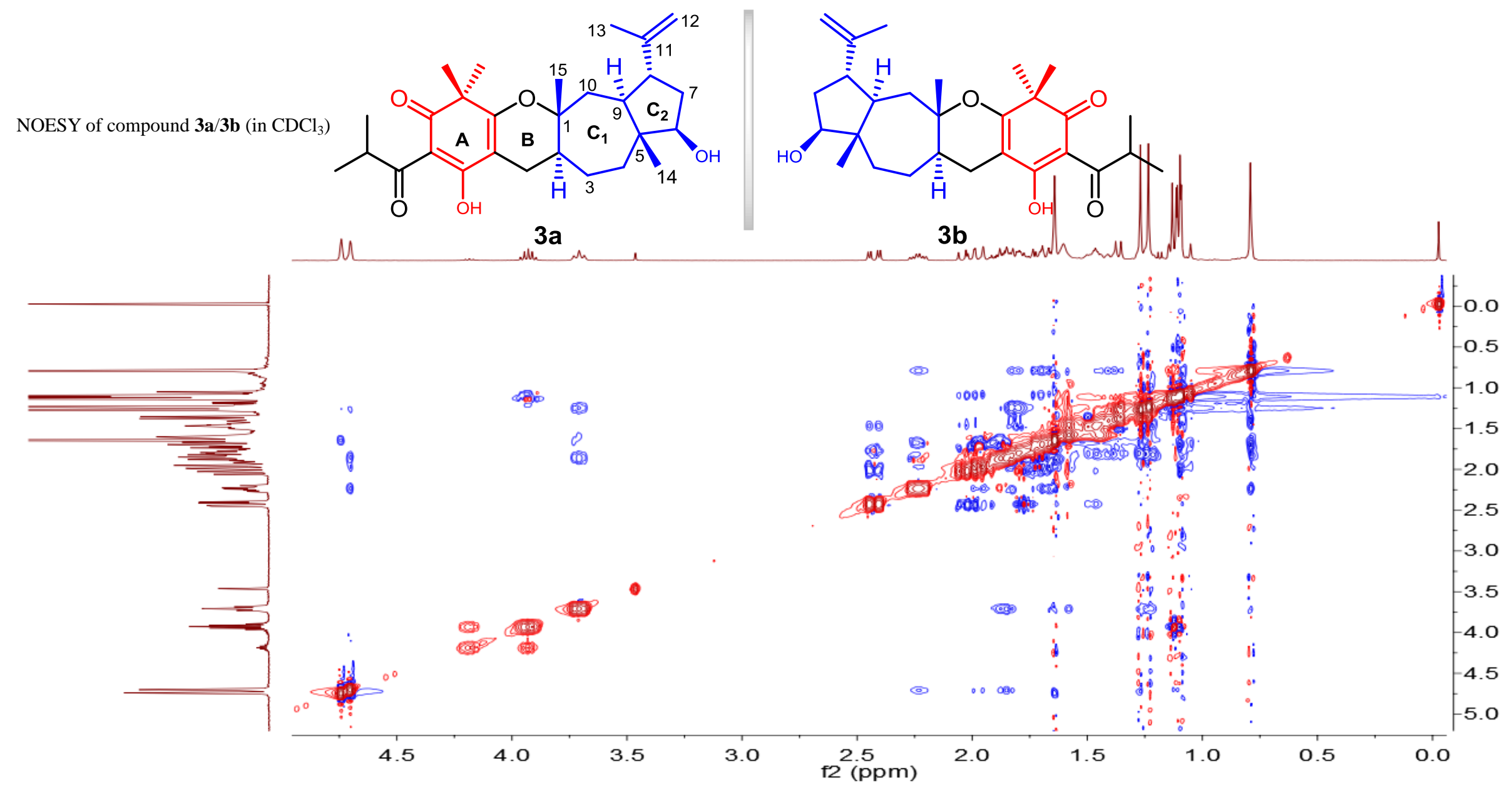


UV of compound $\mathbf{3 a} / \mathbf{3 b}$ (in $\mathrm{MeOH}$ )
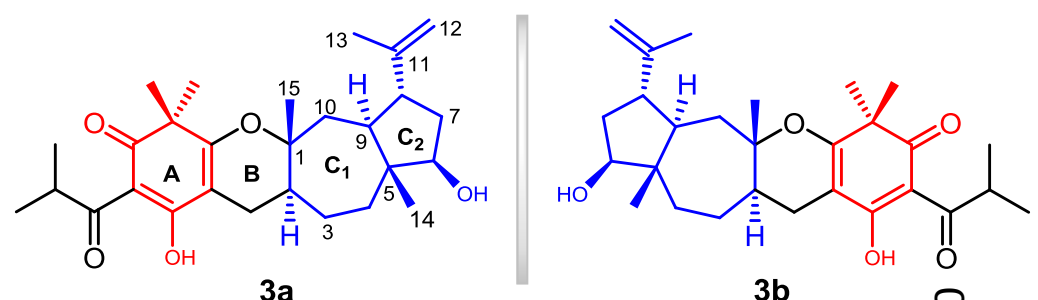

$3 b$

ஜ

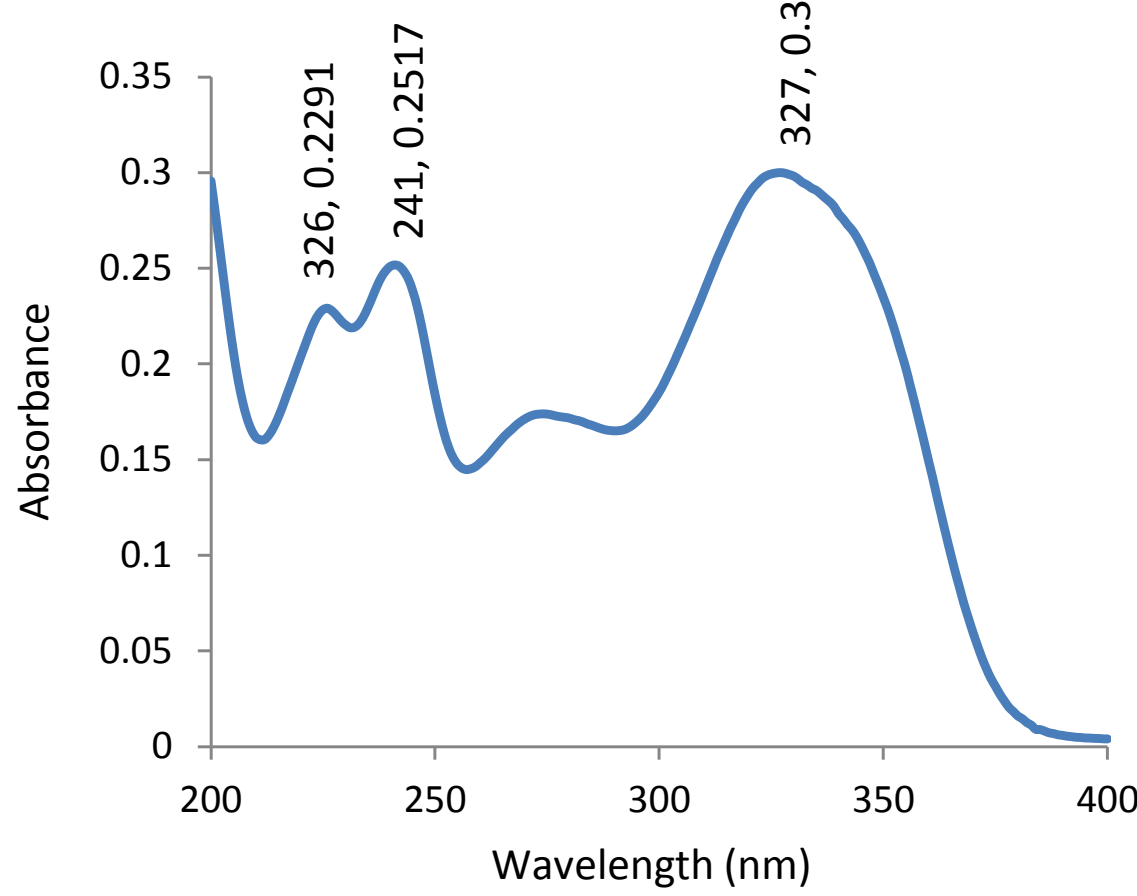




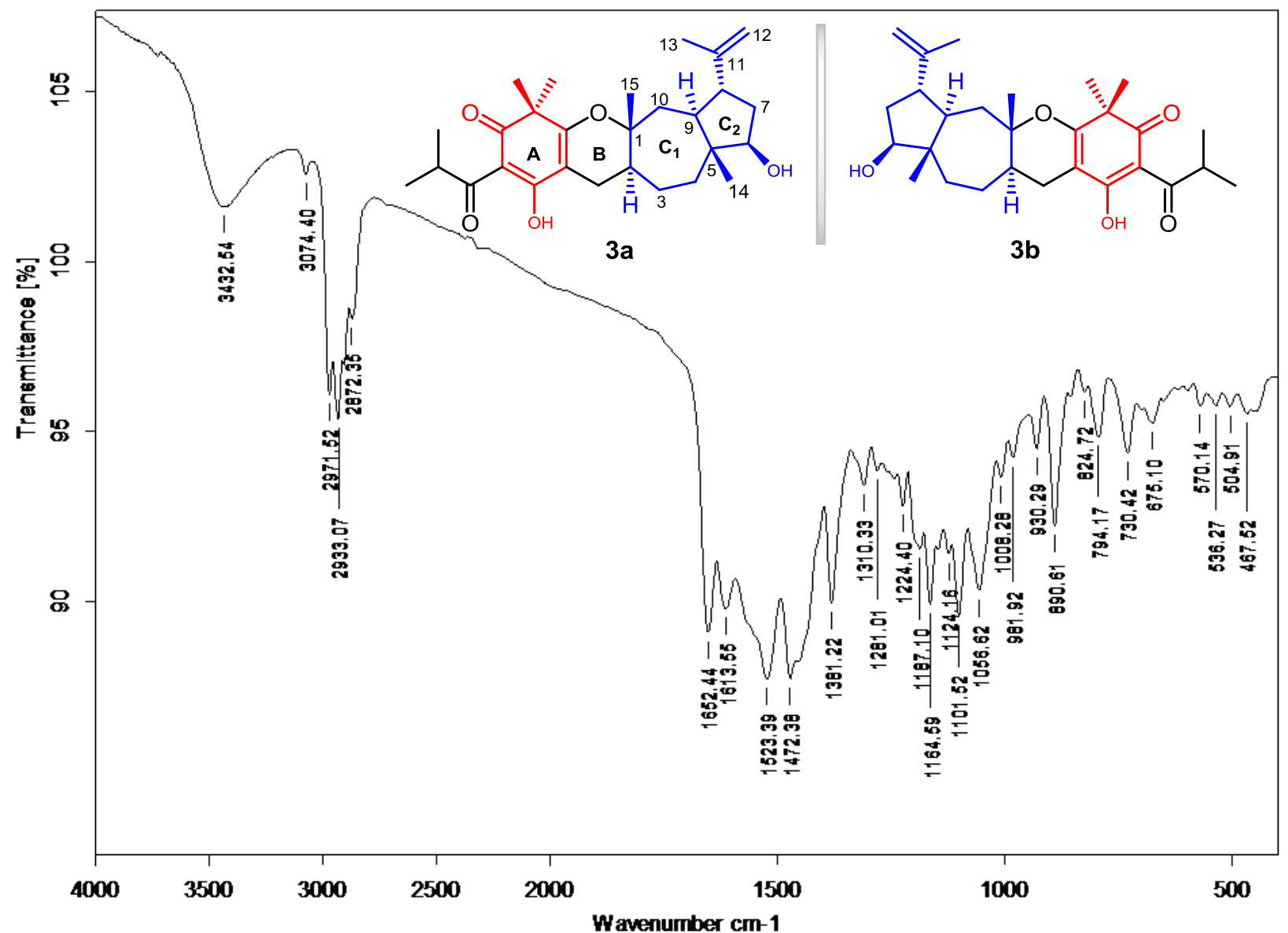


HRESIMS of compound 4

hlz2-12-7-1 \#21 RT: 0.30 AV: 1 NL: 2.23E6 T: FTMS + p ESI Full ms [50.00-1500.00]

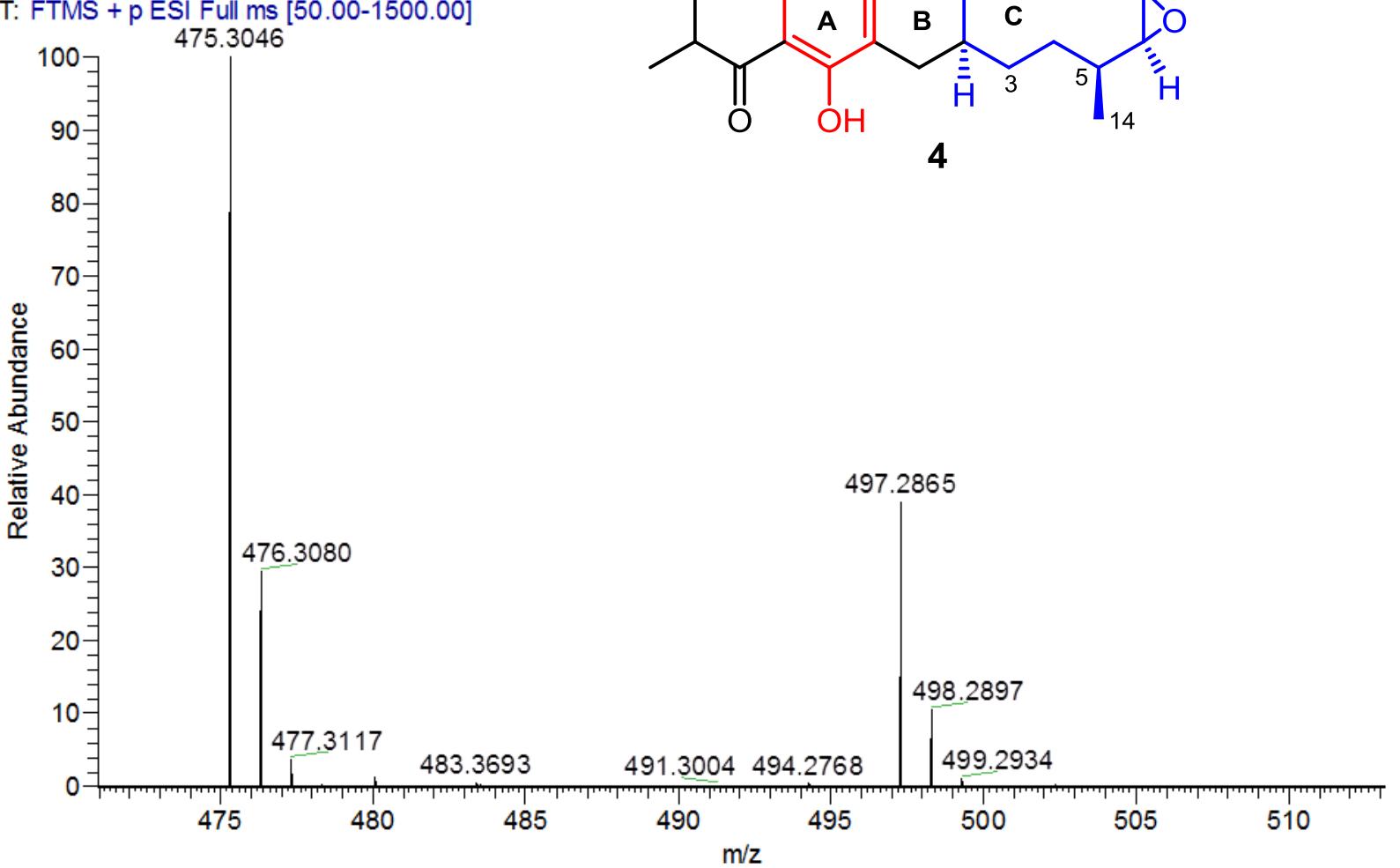


${ }^{1} \mathrm{H}$ NMR of compound 4 (in $\mathrm{CDCl}_{3}$ )

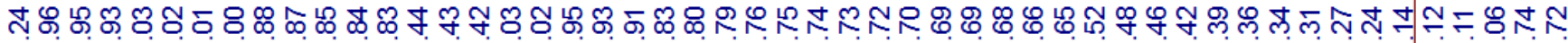

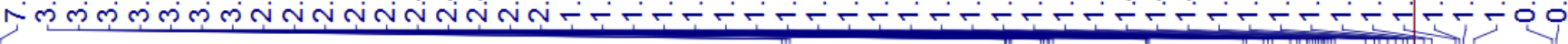
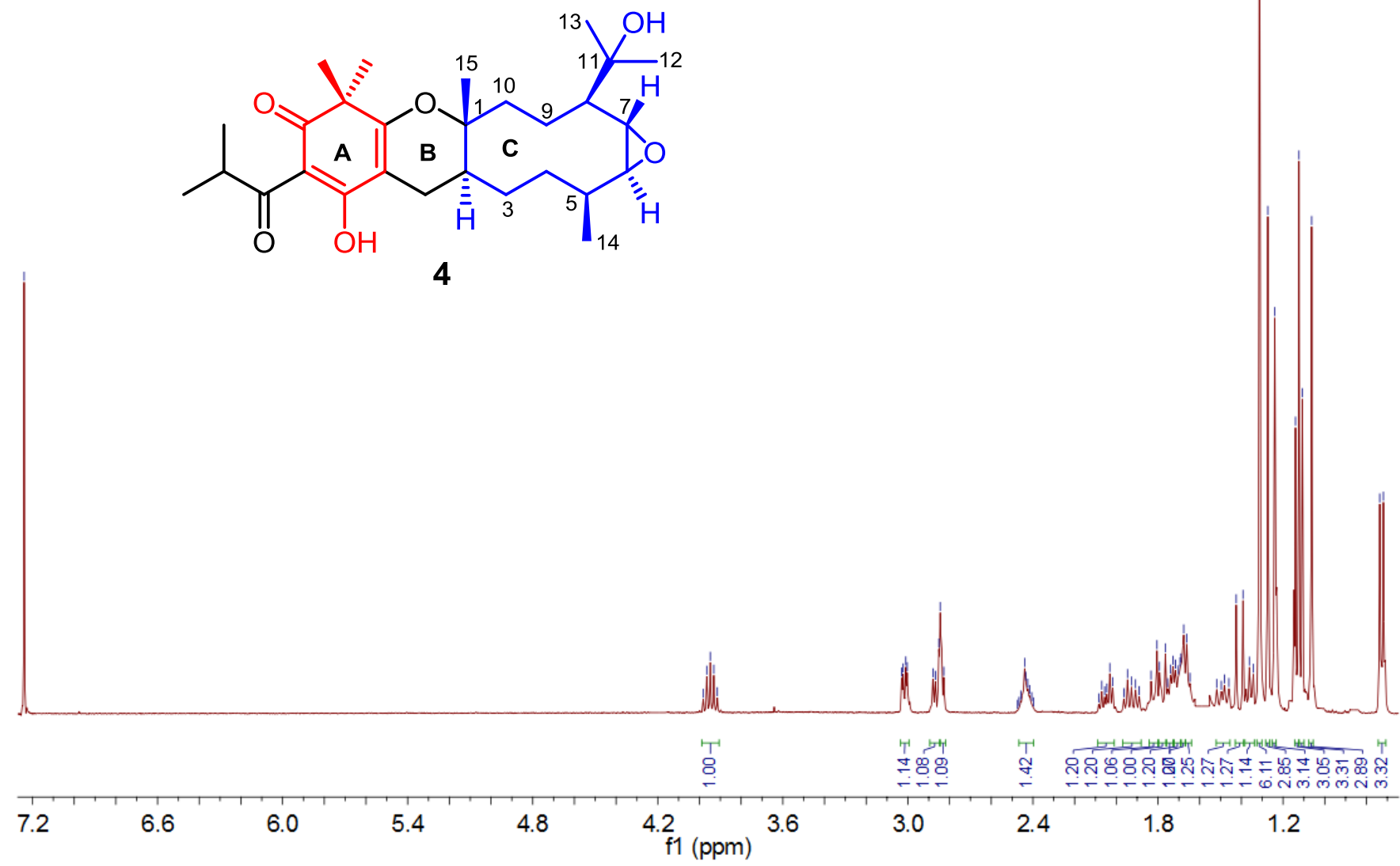


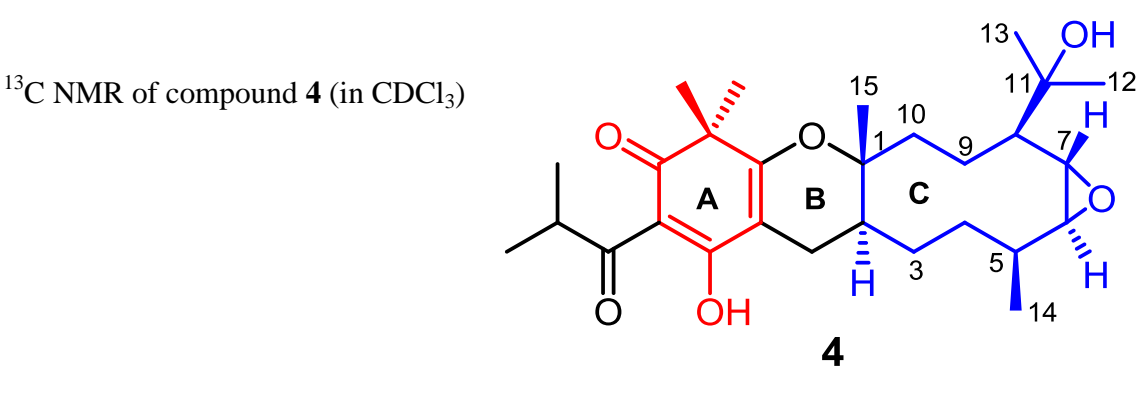

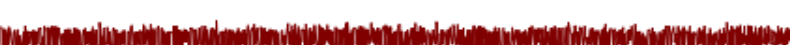

||||||||||

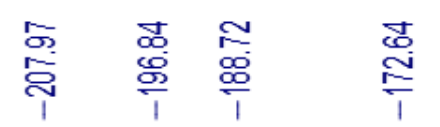
离 छ윰

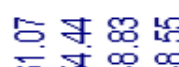

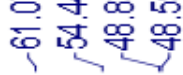

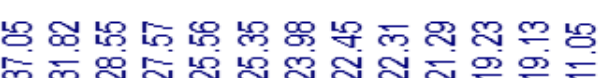
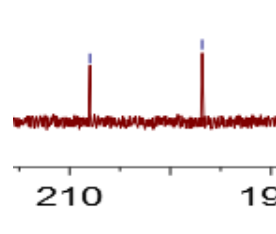

$190 \quad 170$

130

110 f1 (ppm) 90

80

$70 \quad 60$

$50 \quad 40$

20 


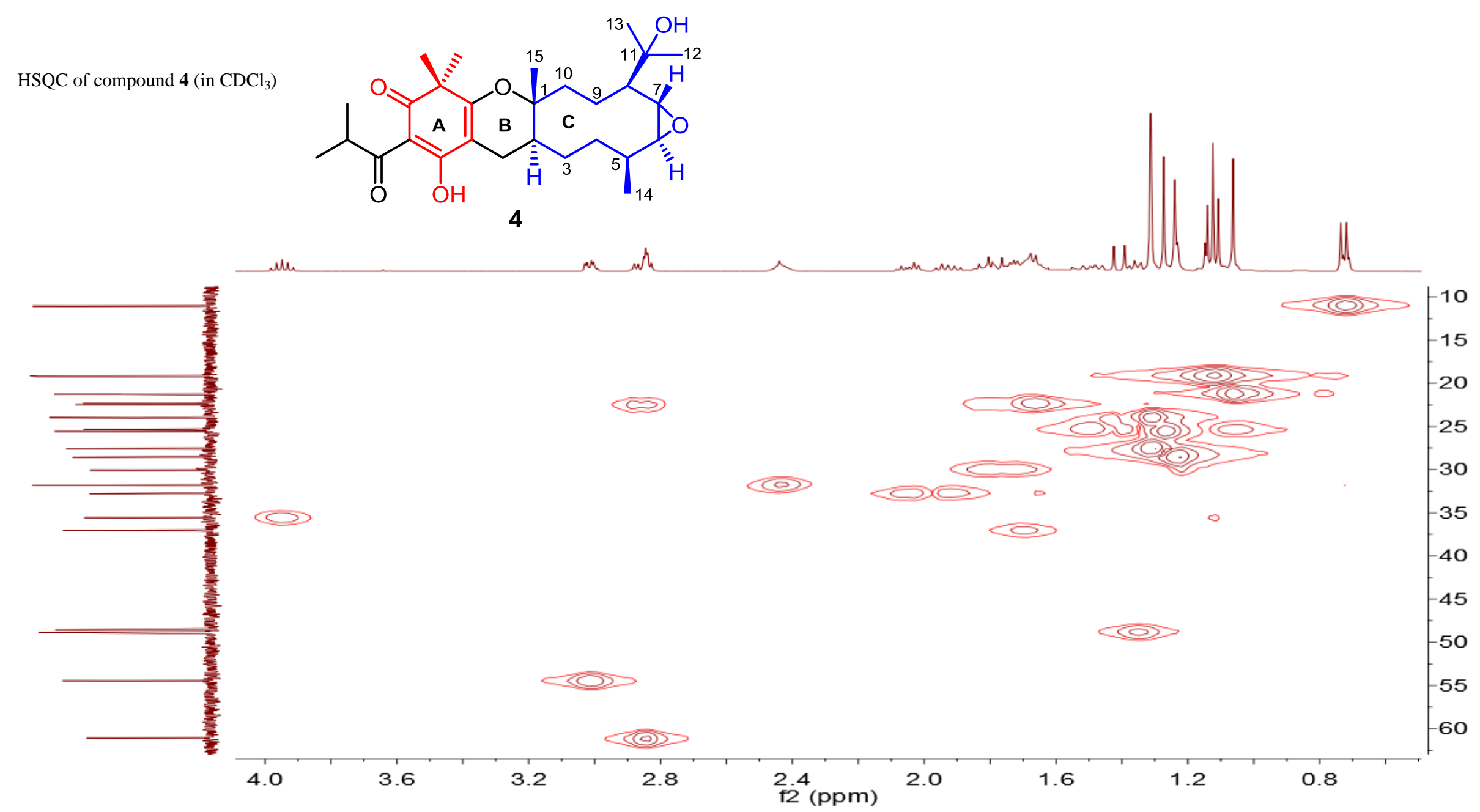




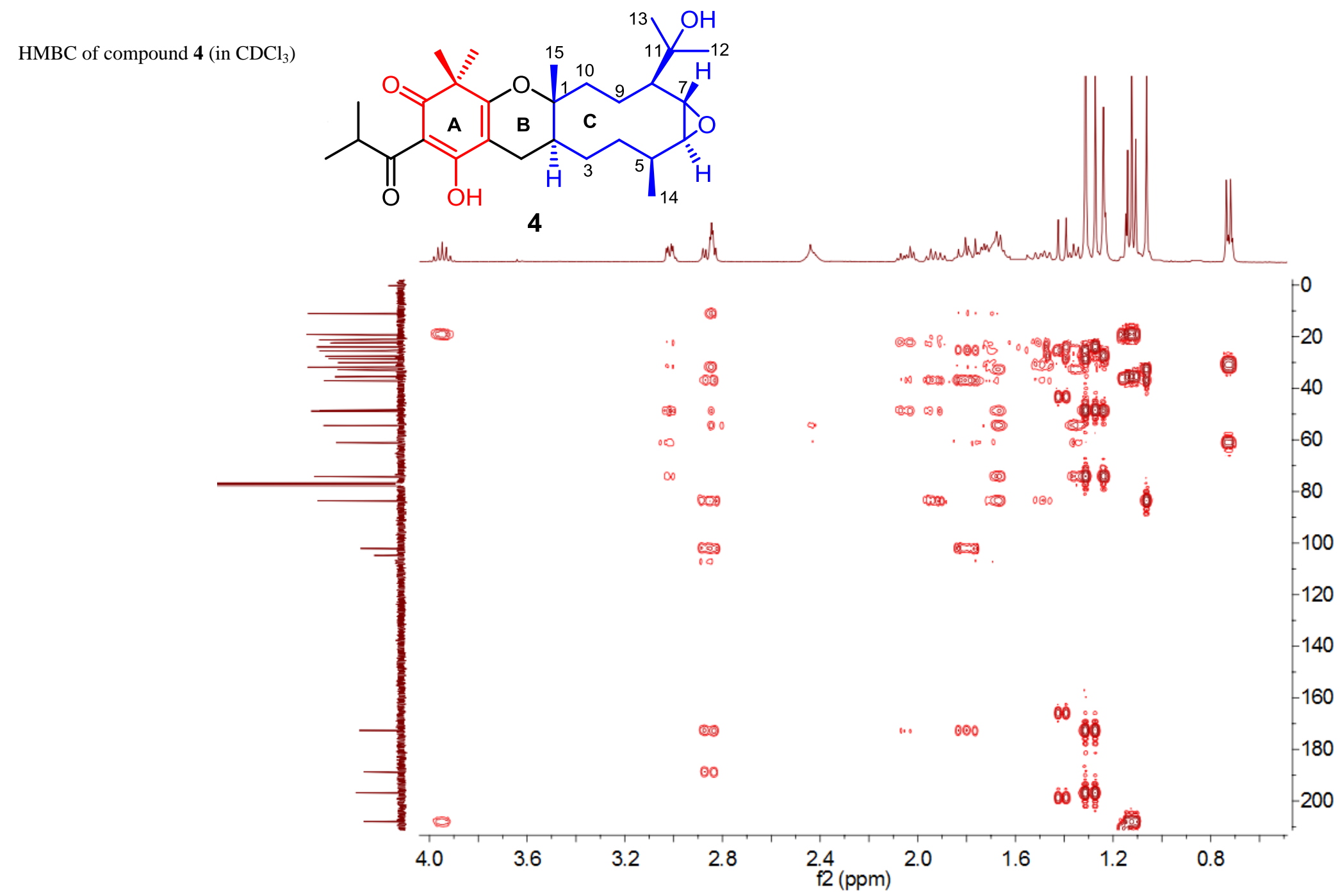




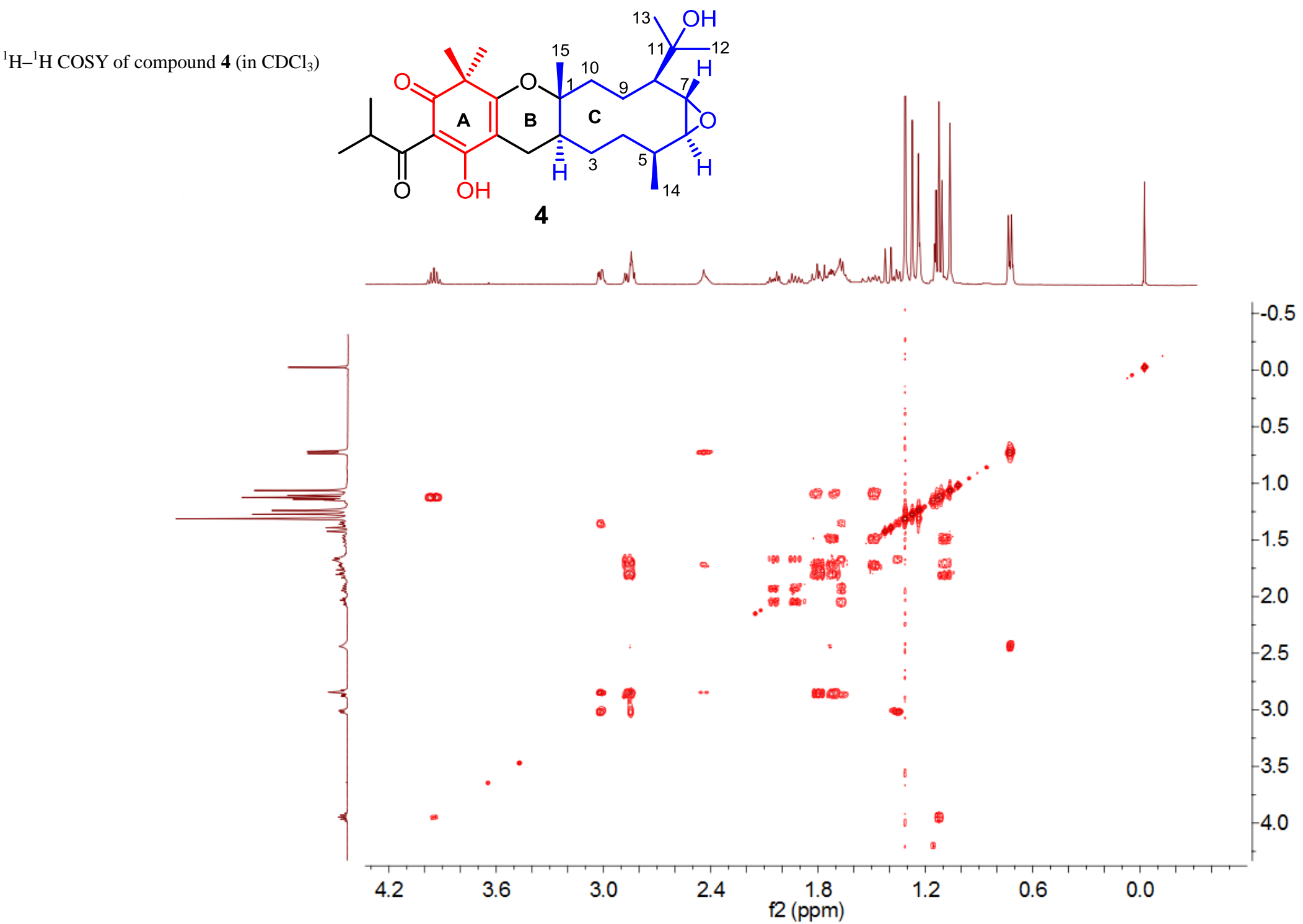




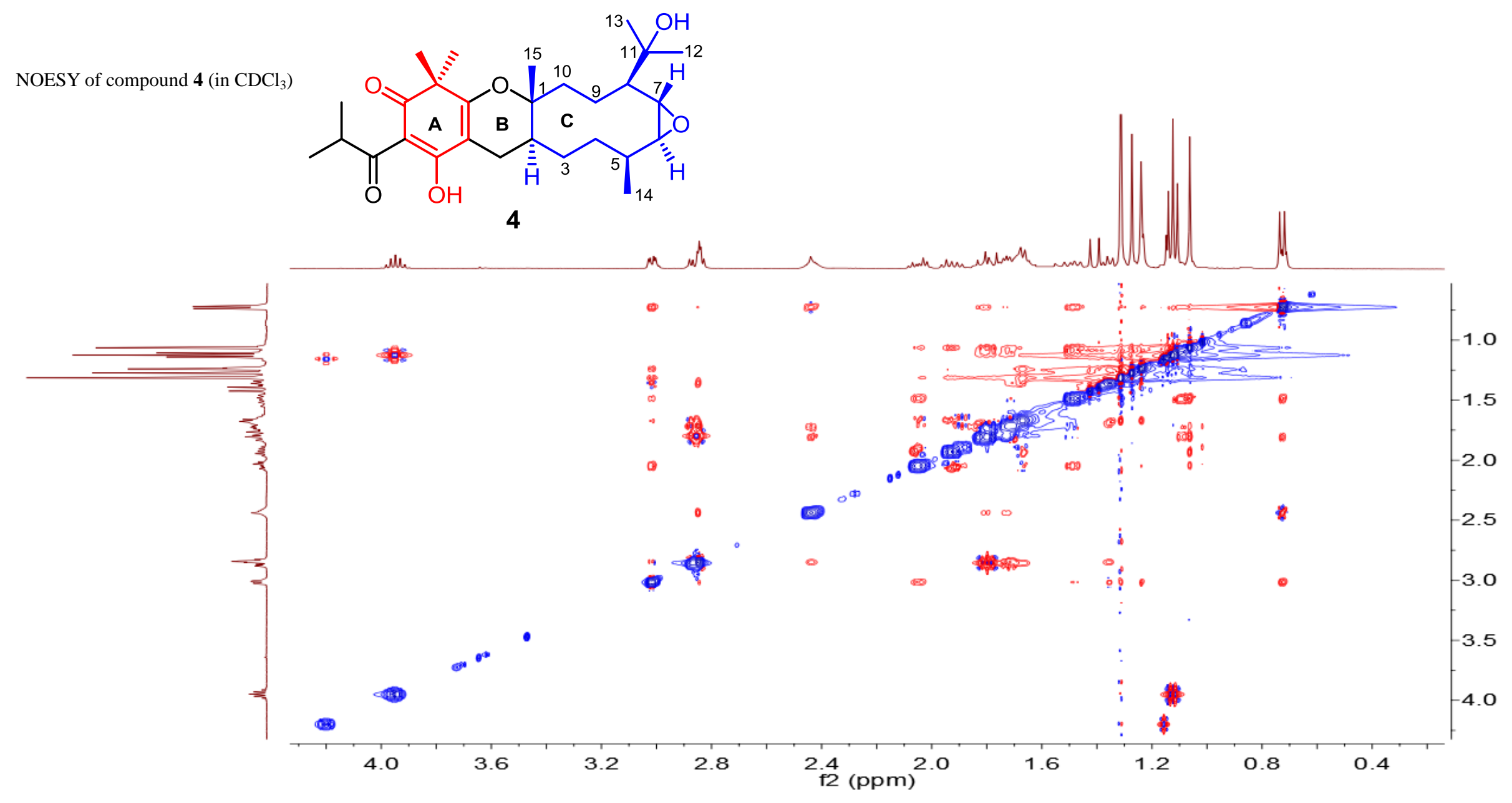


UV of compound 4 (in $\mathrm{MeOH}$ )

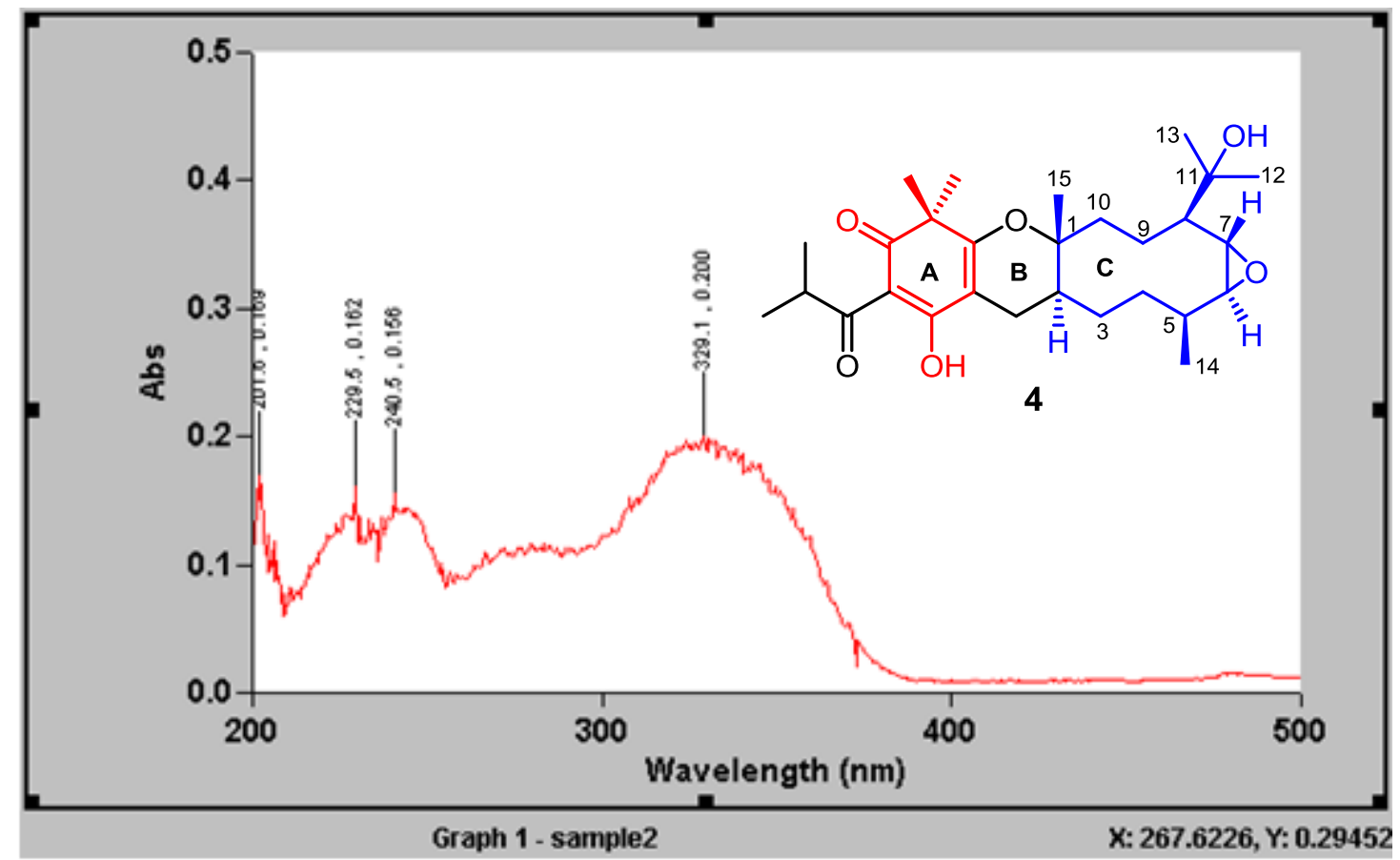


IR of compound 4 ( $\mathrm{KBr}$ disc)

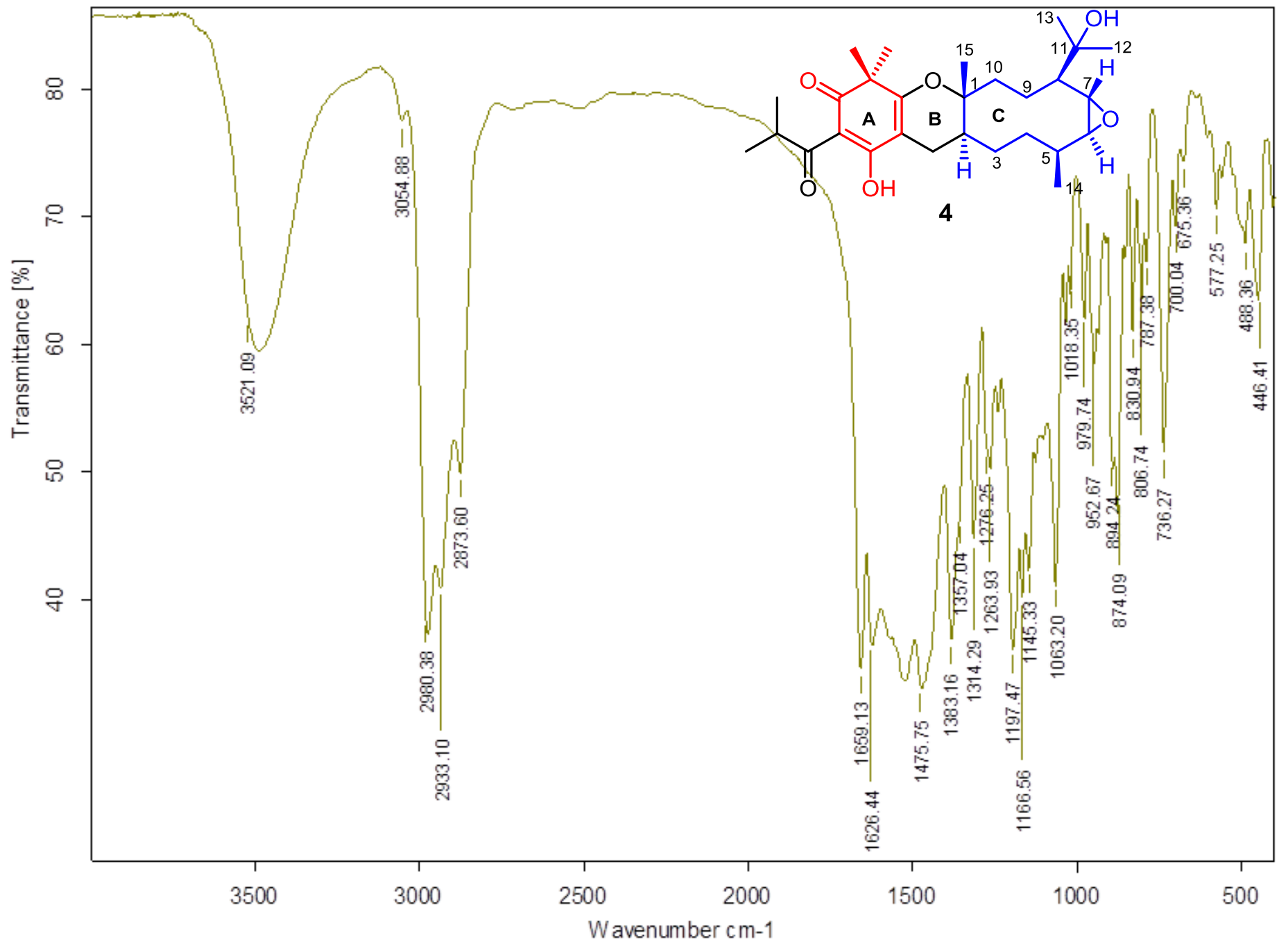


HRESIMS of compound $\mathbf{5}$

hlz2-56-2 \#9 RT: 0.13 AV: 1 NL: 1.16E7 T: FTMS + p ESI Full ms [50.00-1500.00]

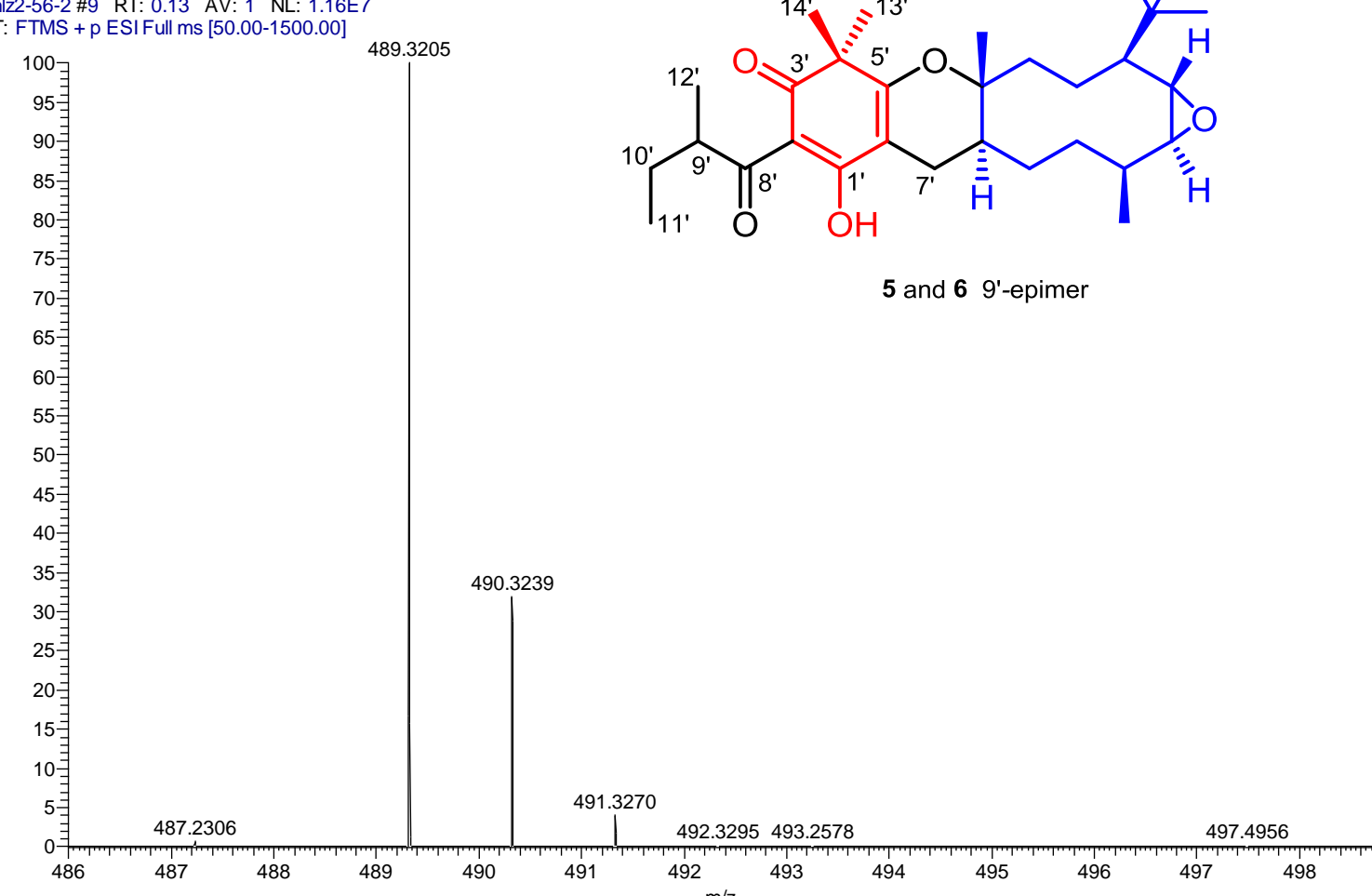


${ }^{1} \mathrm{H}$ NMR of compound 5 (in $\mathrm{CDCl}_{3}$ )

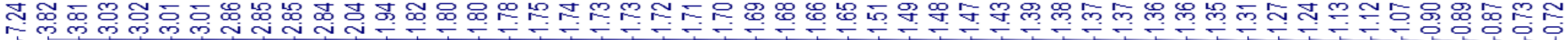

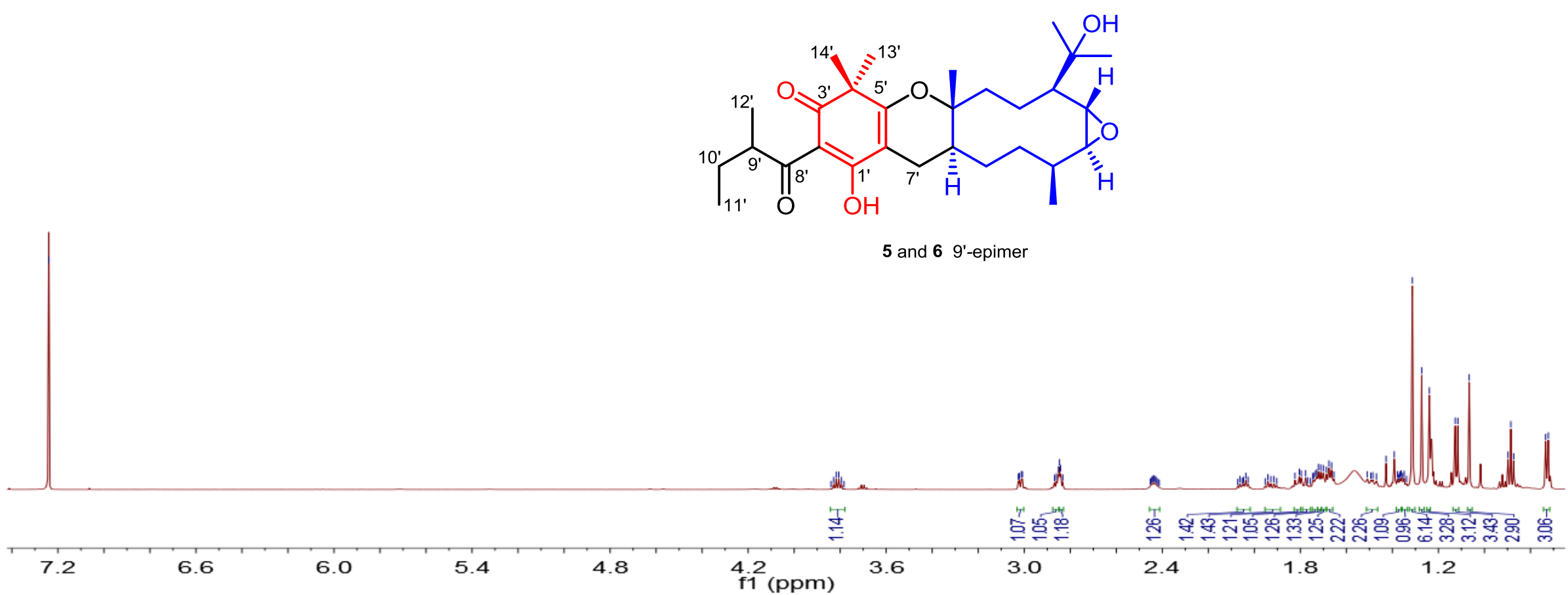


${ }^{13} \mathrm{C}$ NMR of compound 5 (in $\mathrm{CDCl}_{3}$ )

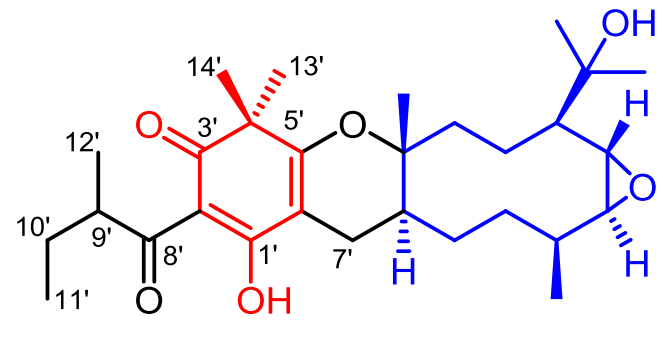

5 and 6 9'-epimer

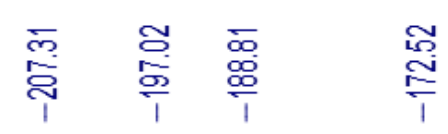

每

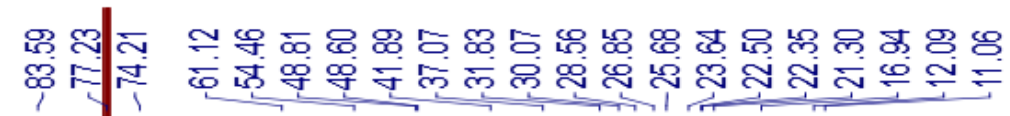

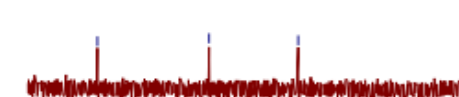

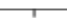

190

170

150

130

90

$80 \quad 70$

60

5040 
HSQC of compound $\mathbf{5}$ (in $\mathrm{CDCl}_{3}$ )
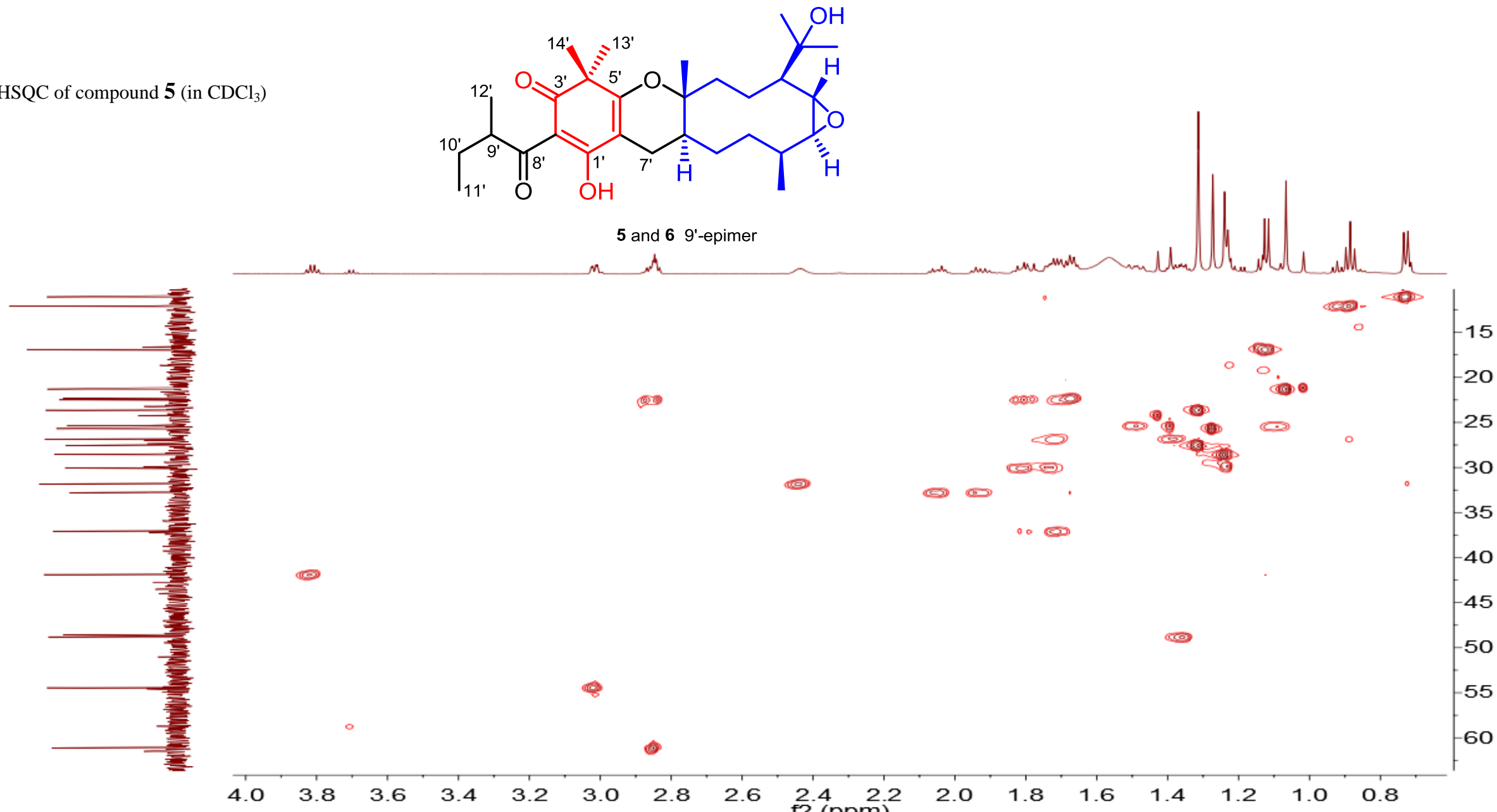
HMBC of compound 5 (in $\mathrm{CDCl}_{3}$ )

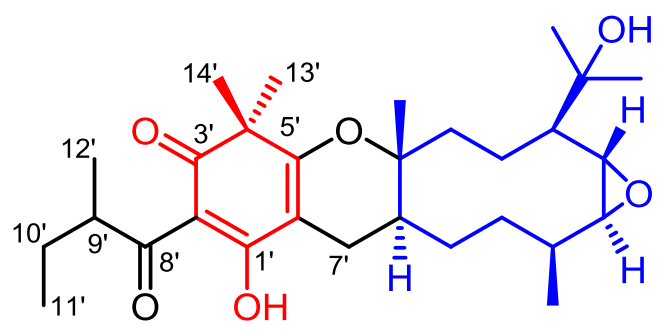

5 and 6 9'-epimer
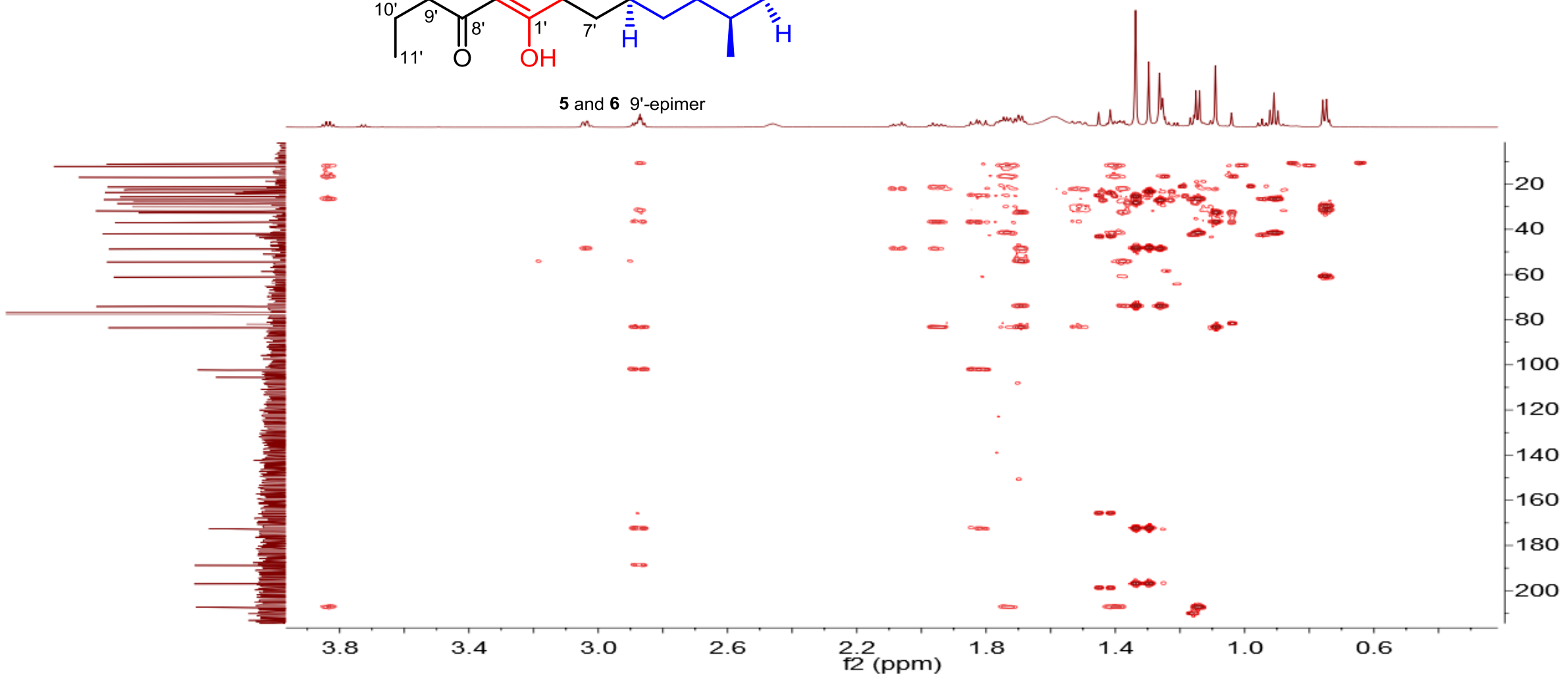
${ }^{1} \mathrm{H}-{ }^{1} \mathrm{H}$ COSY of compound 5 (in $\mathrm{CDCl}_{3}$ )
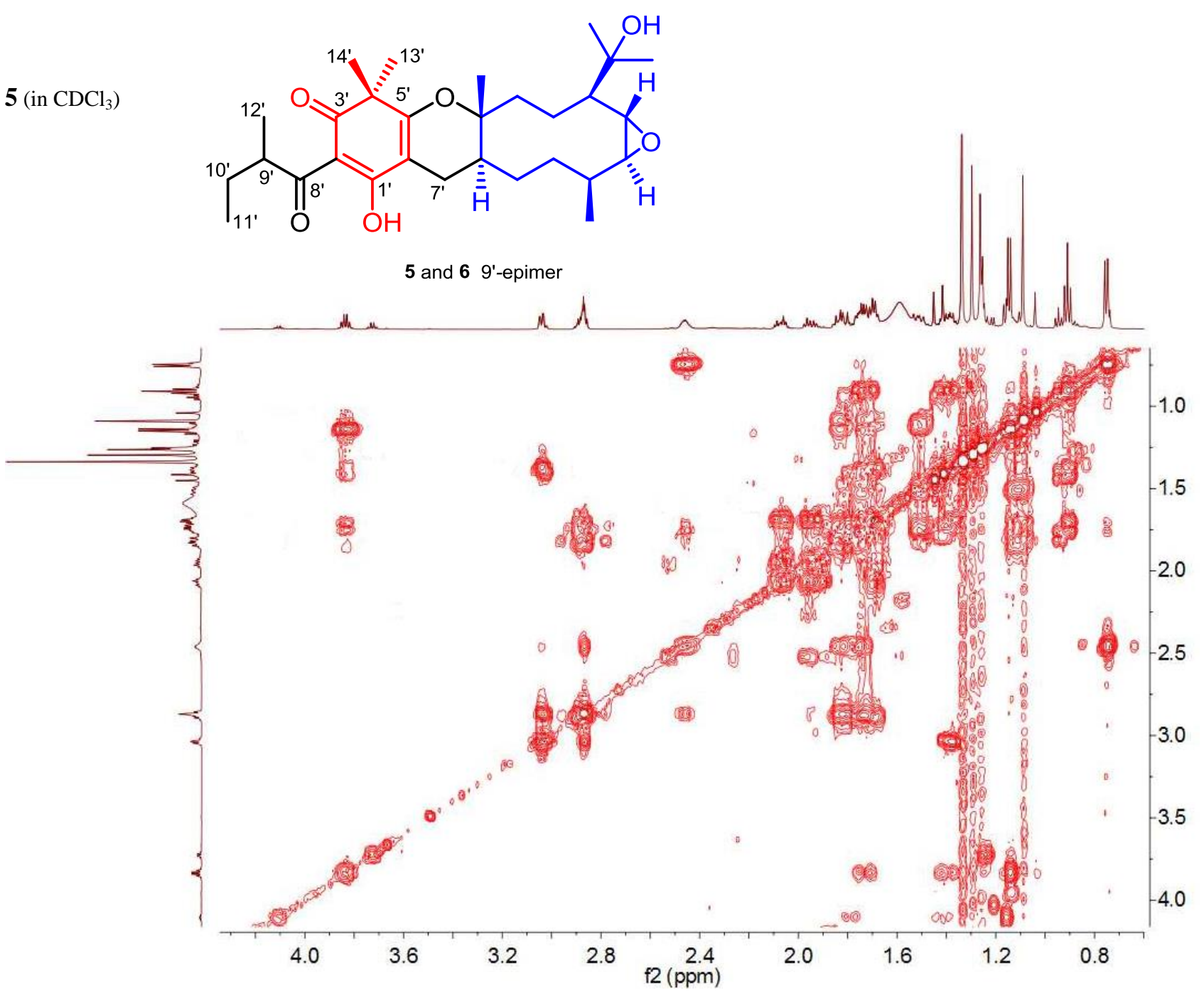


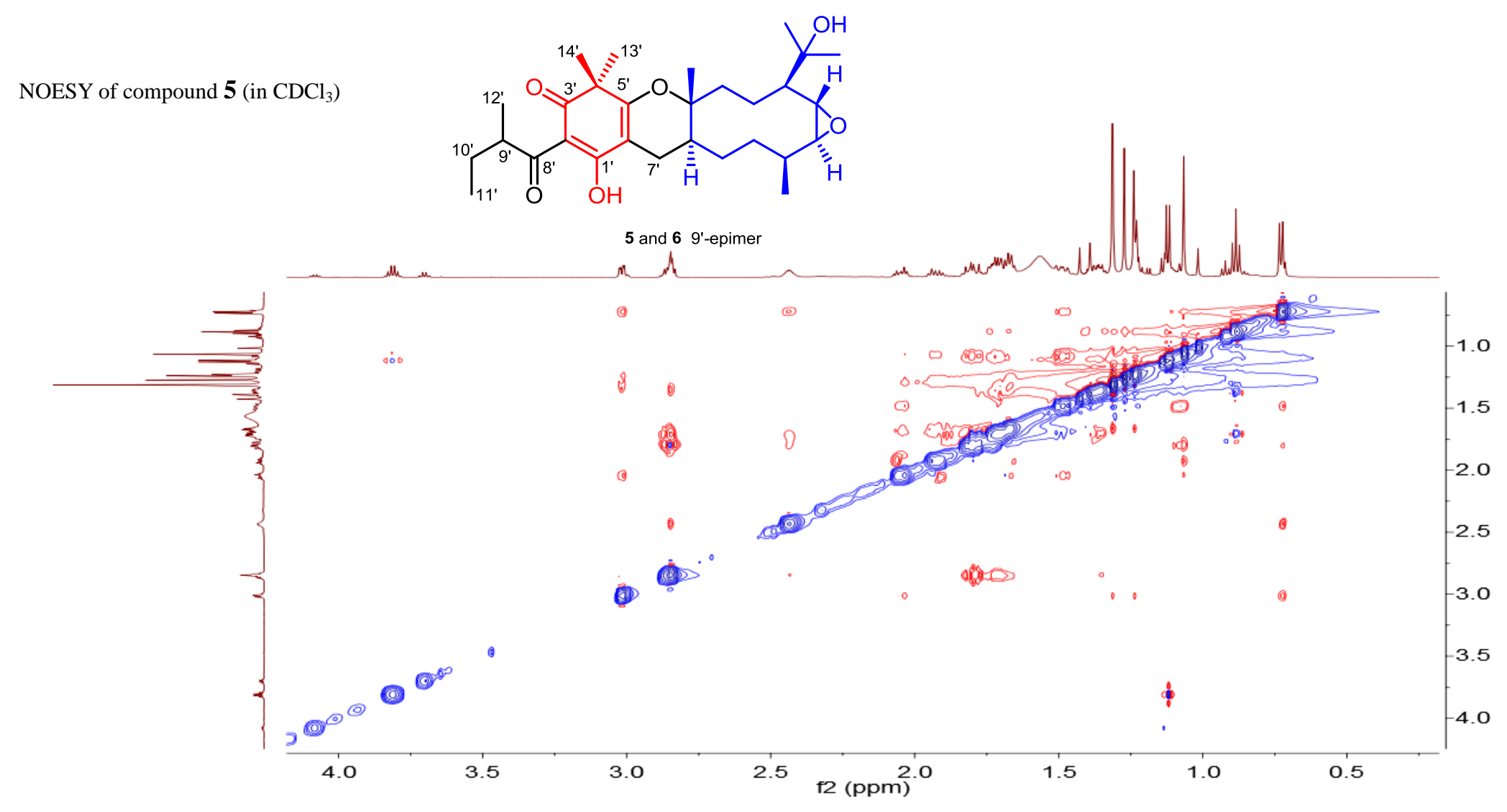


UV of compound 5 (in $\mathrm{MeOH}$ )
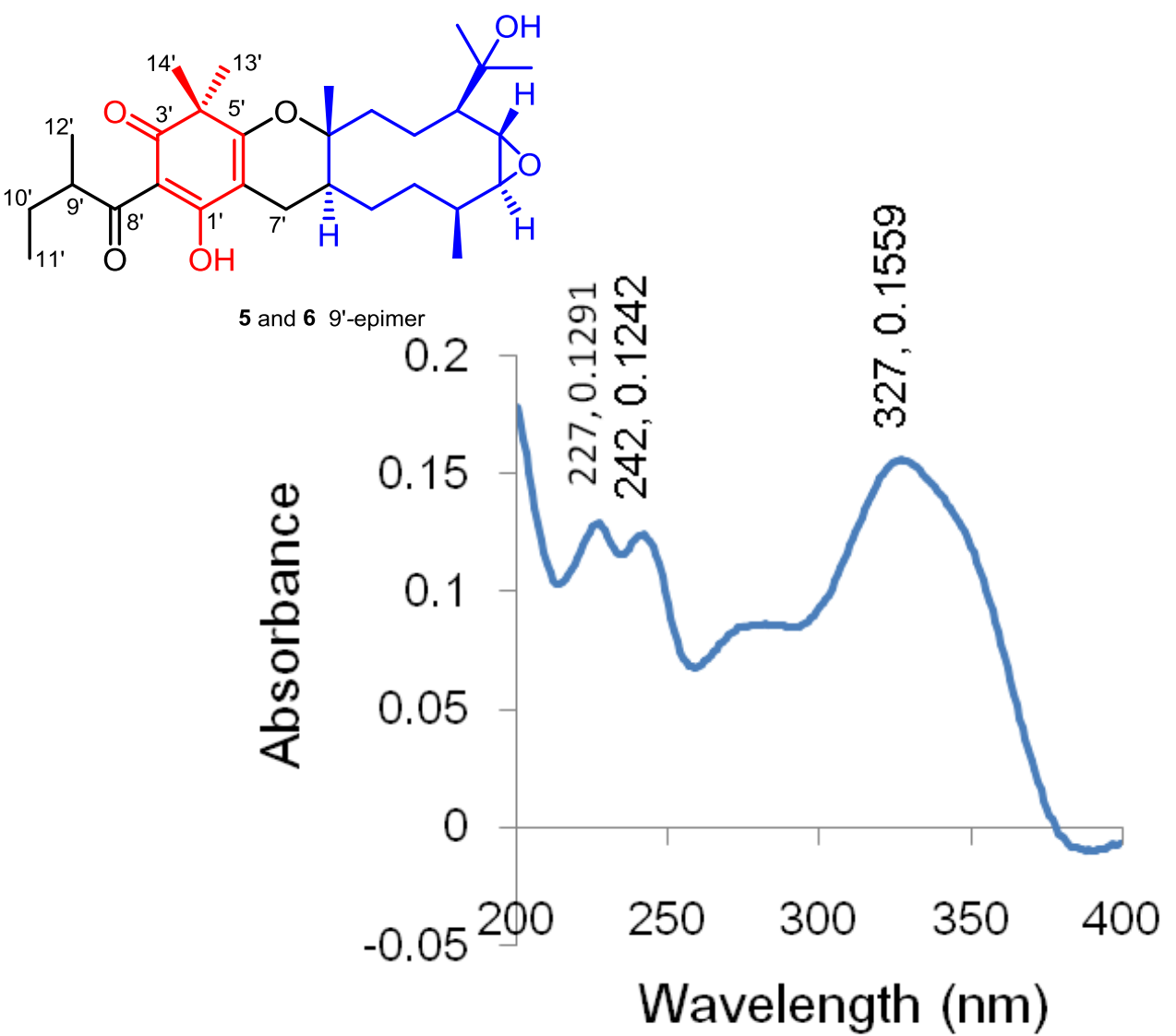
IR of compound $\mathbf{5}$ (KBr disc)

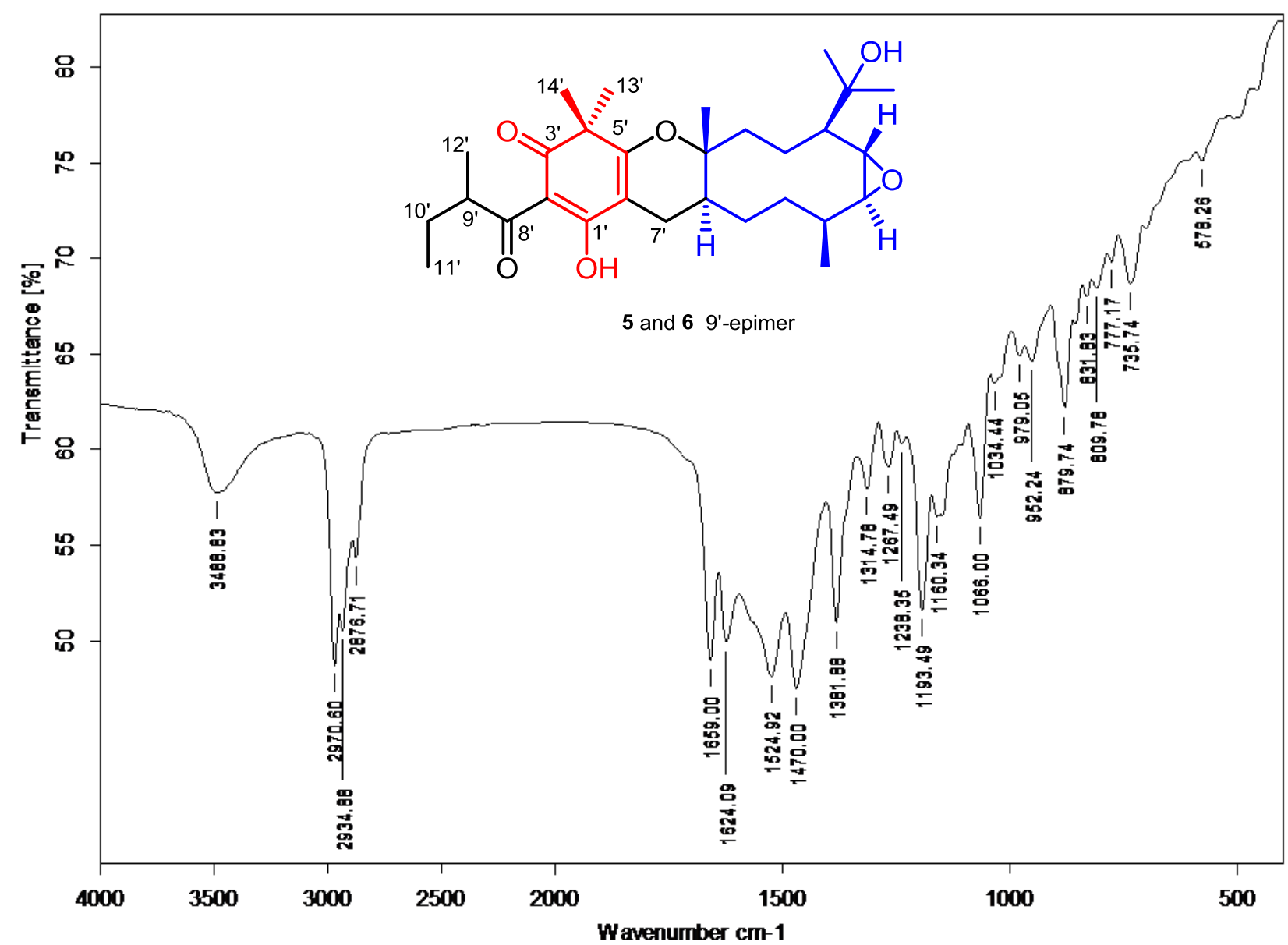


HRESIMS of compound $\mathbf{6}$

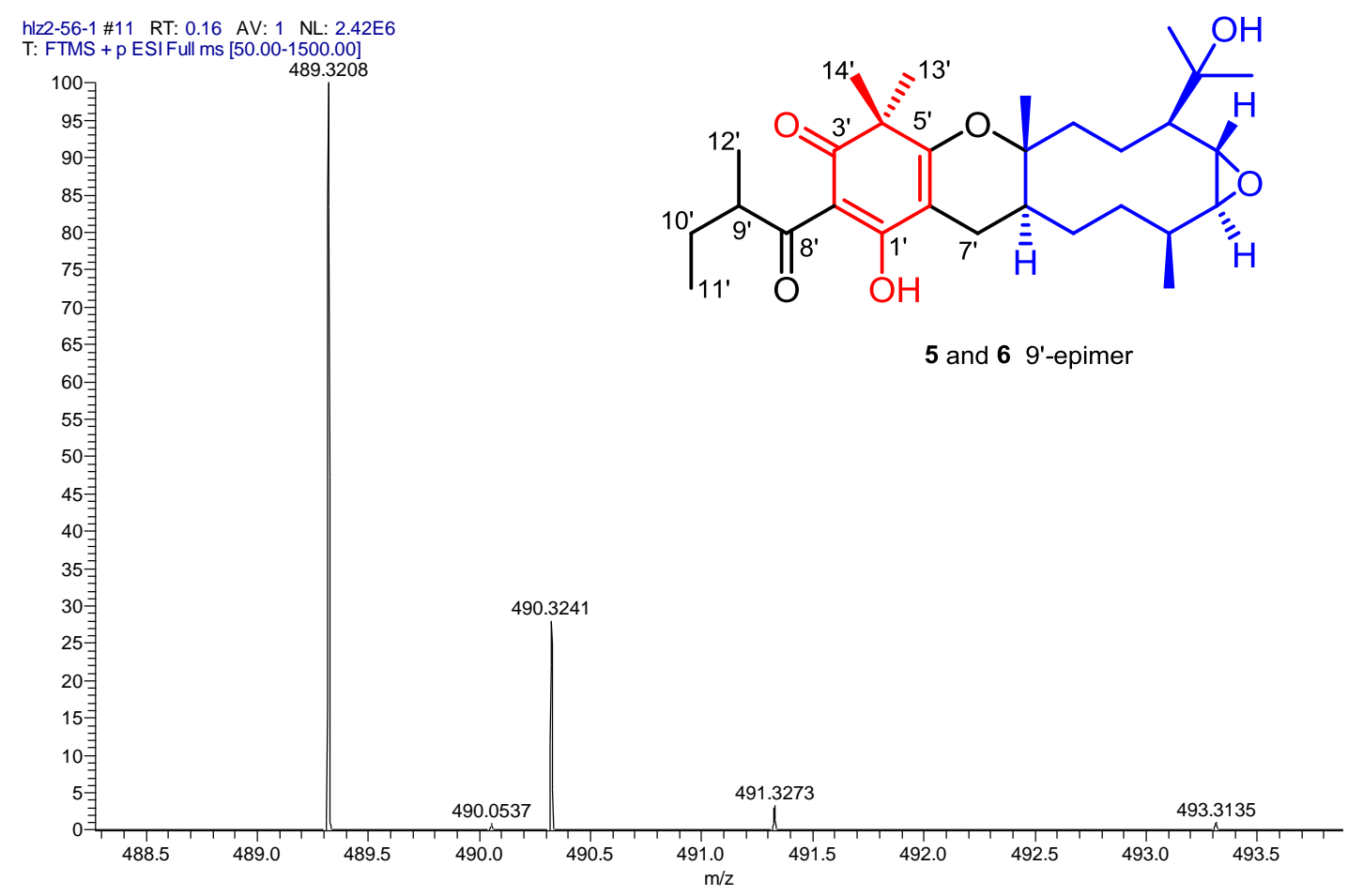


${ }^{1} \mathrm{H}$ NMR of compound 6 (in $\mathrm{CDCl}_{3}$ )

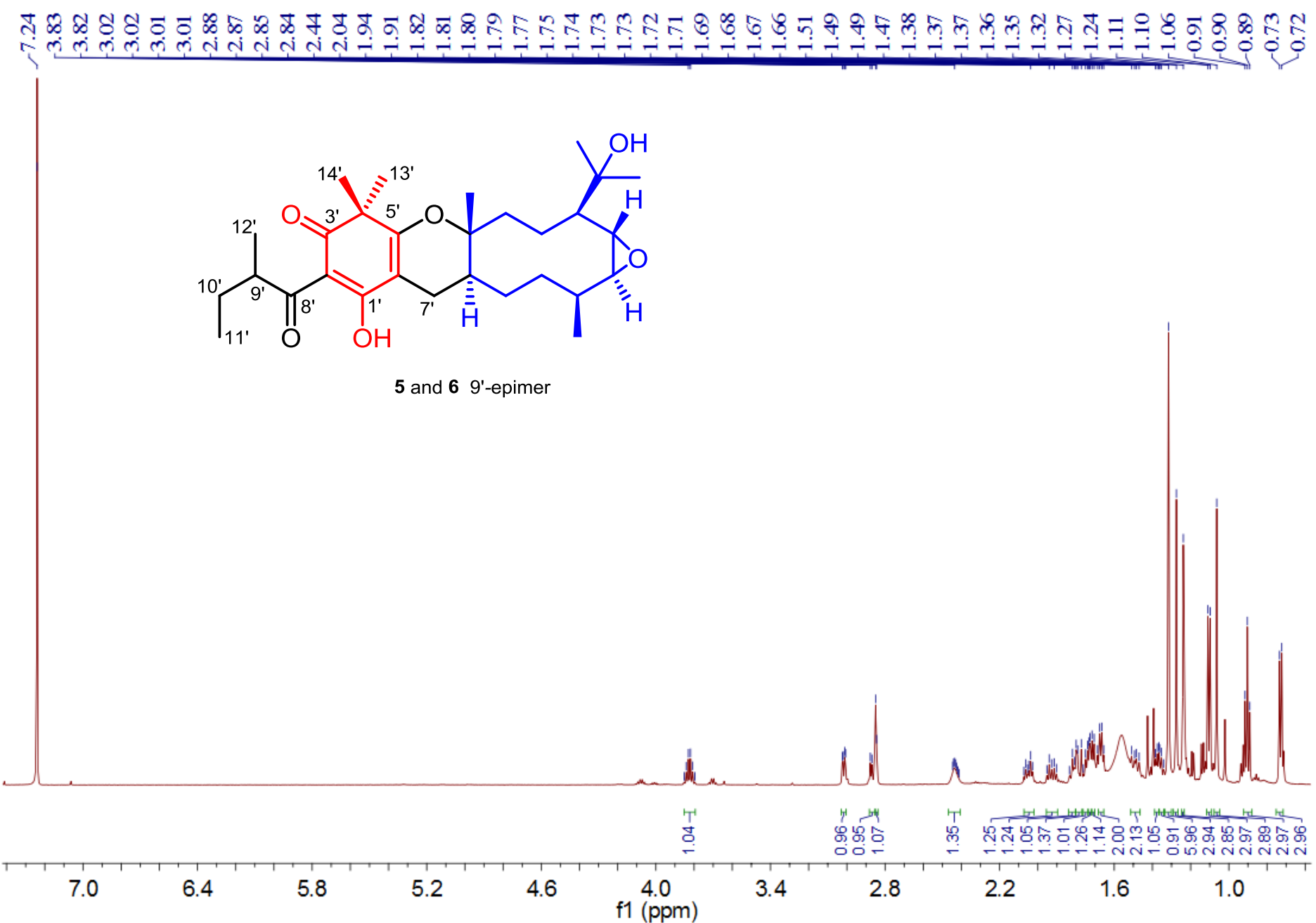




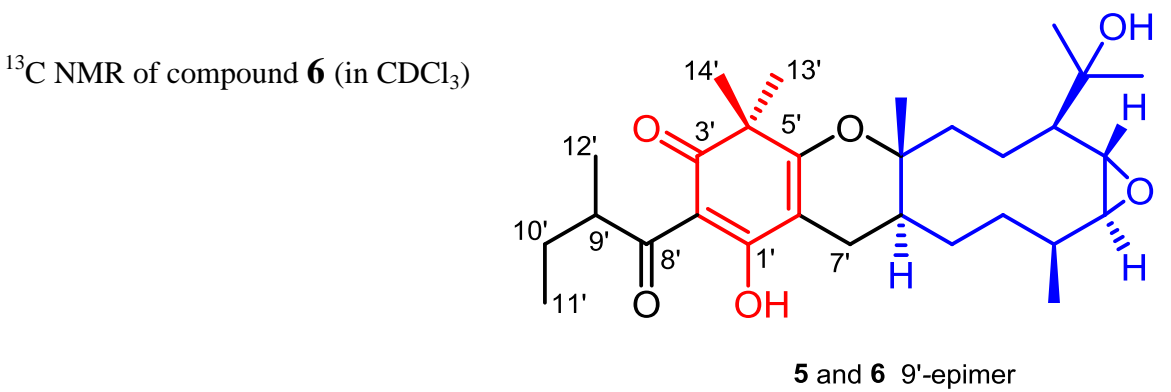

5 and 6 9'-epimer

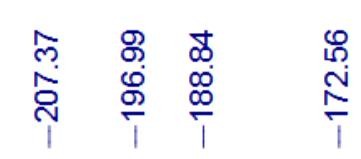

దำ

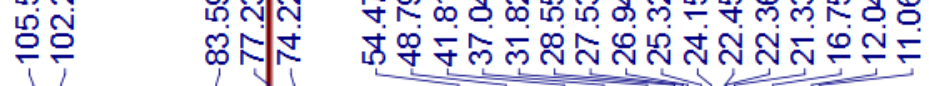

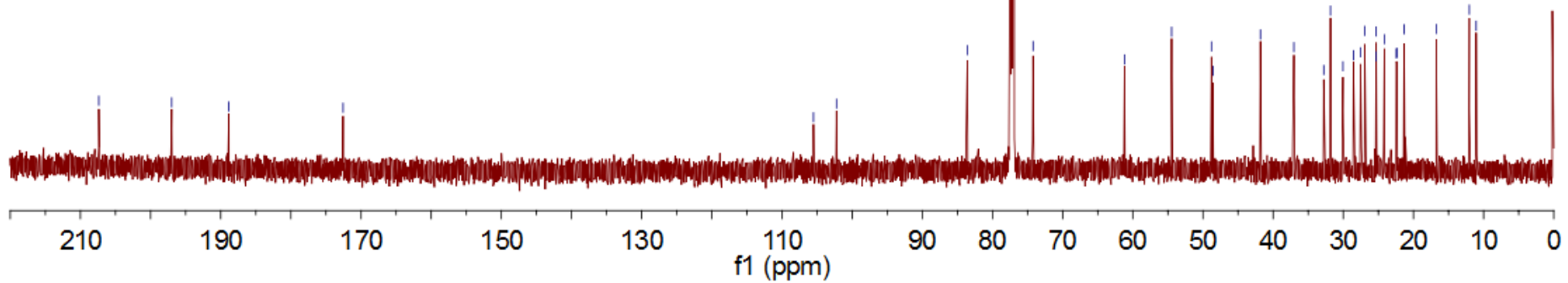




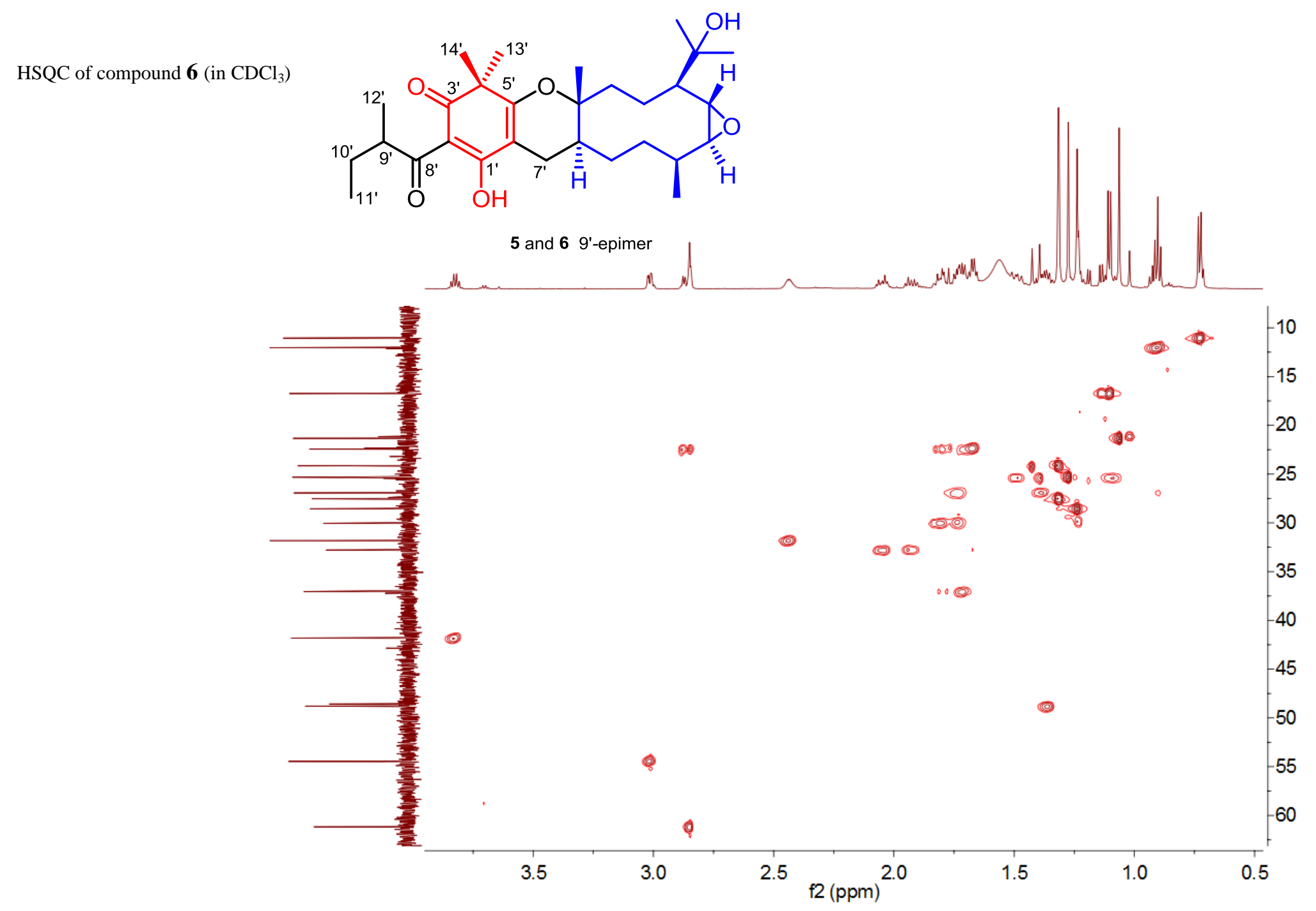




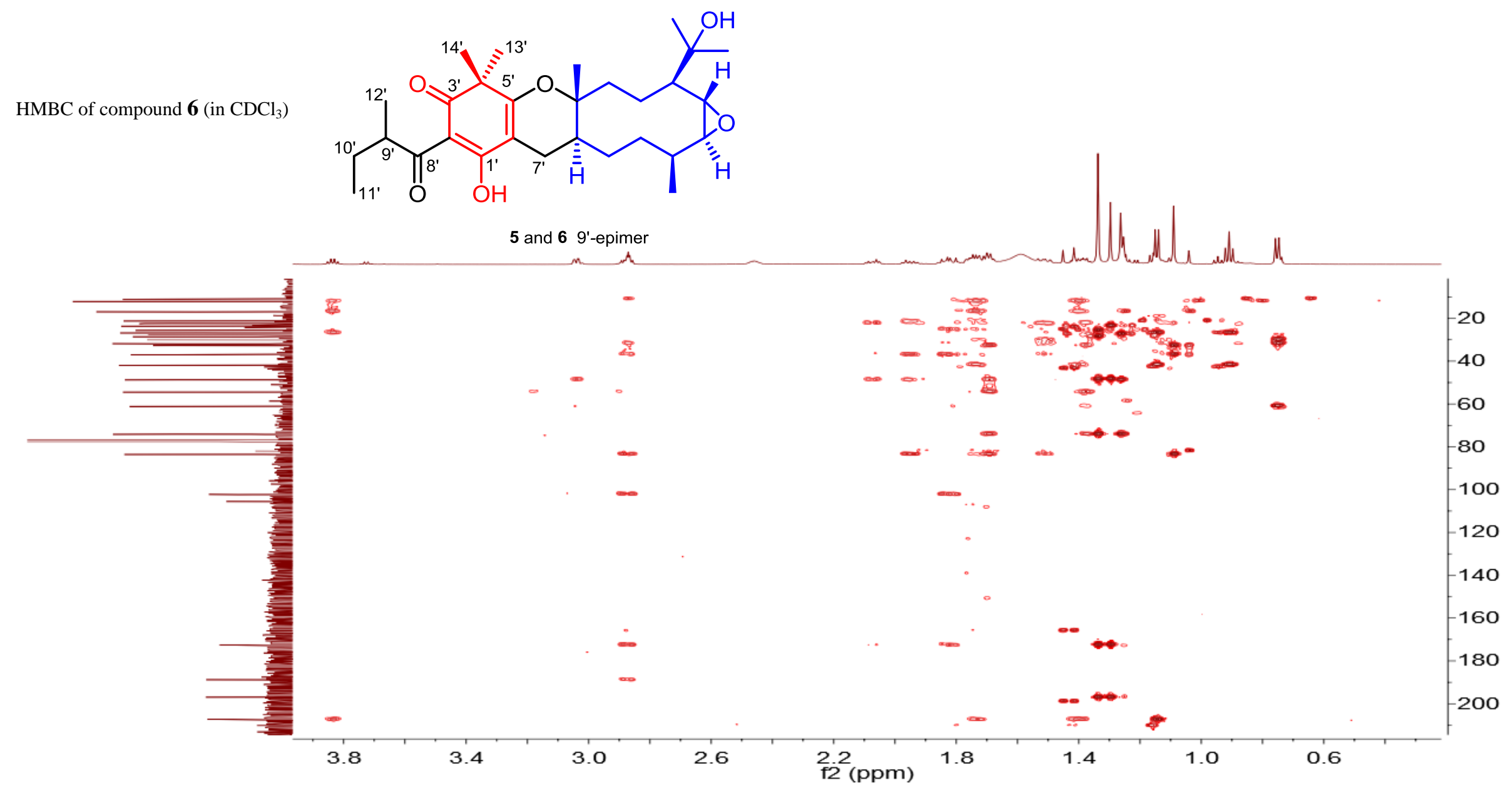




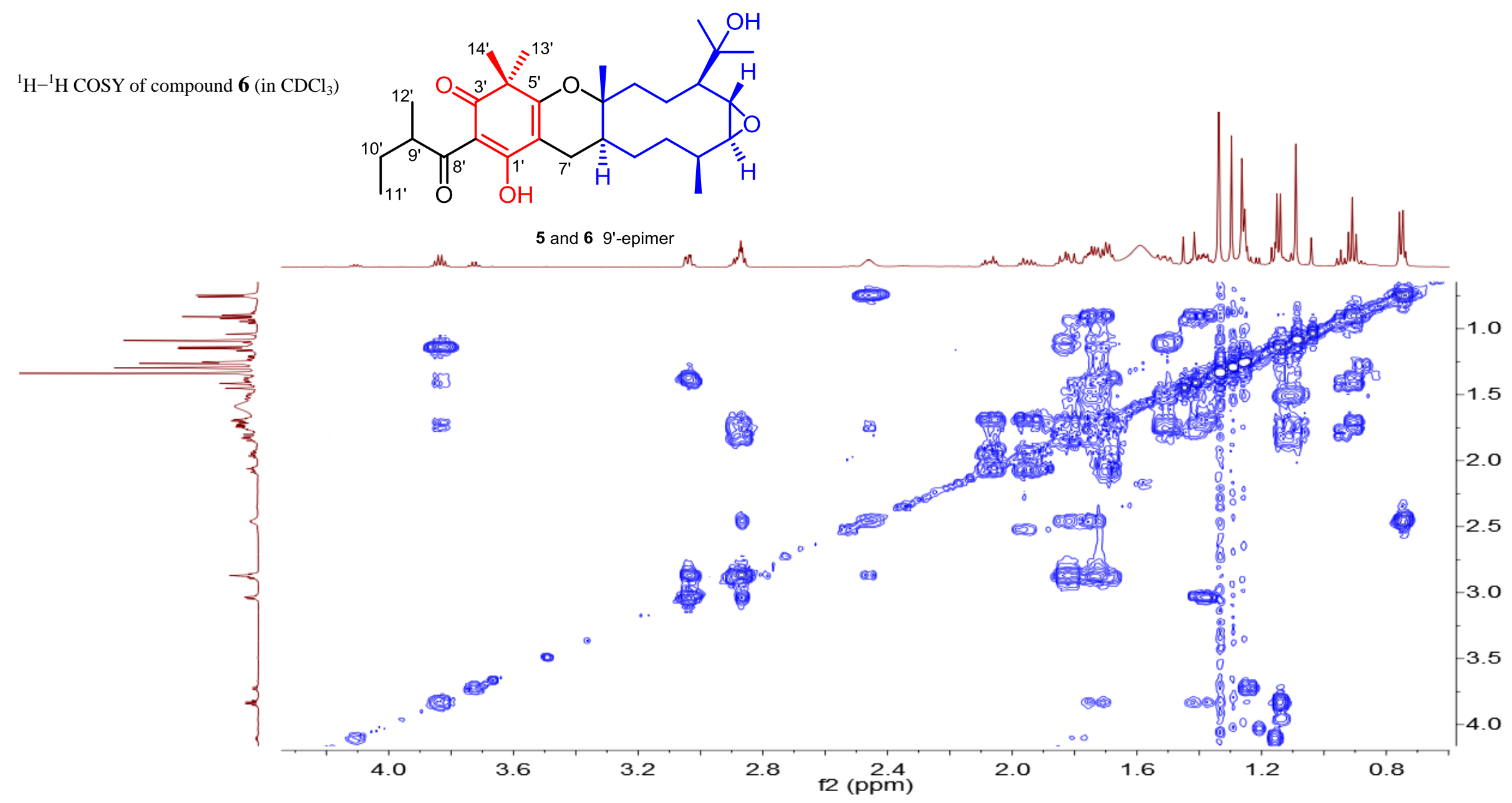




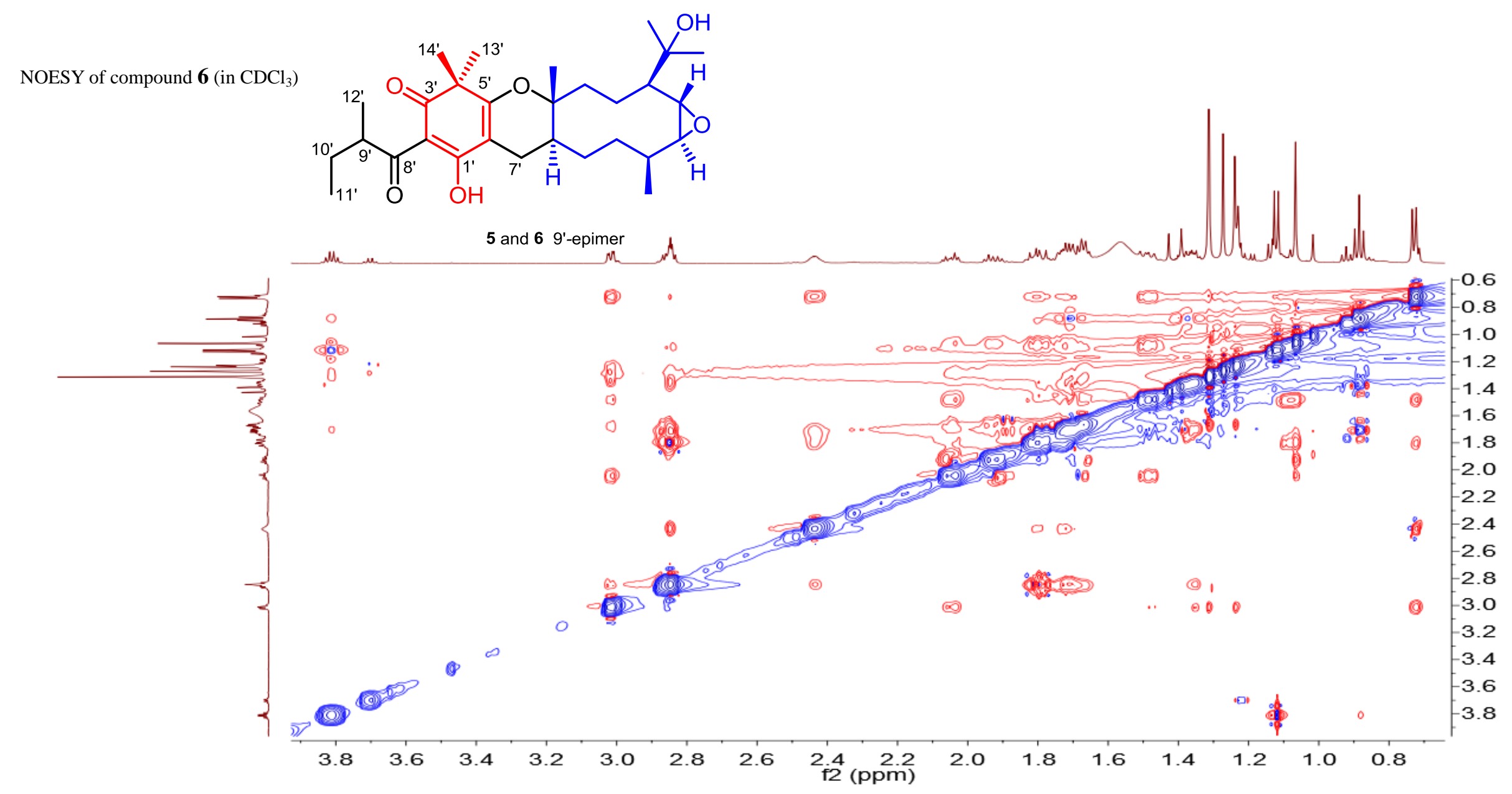




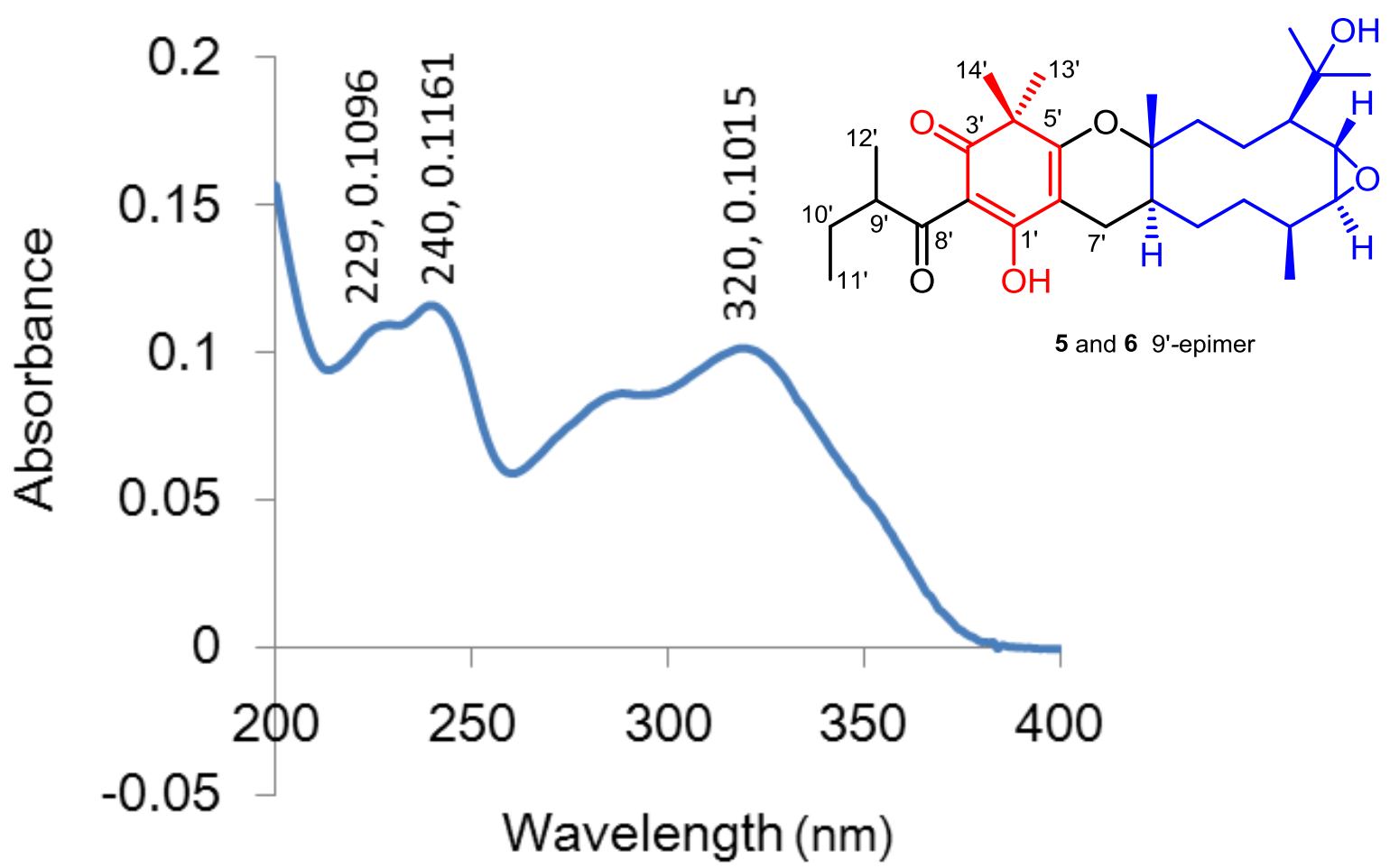


IR of compound $6(\mathrm{KBr}$ disc $)$

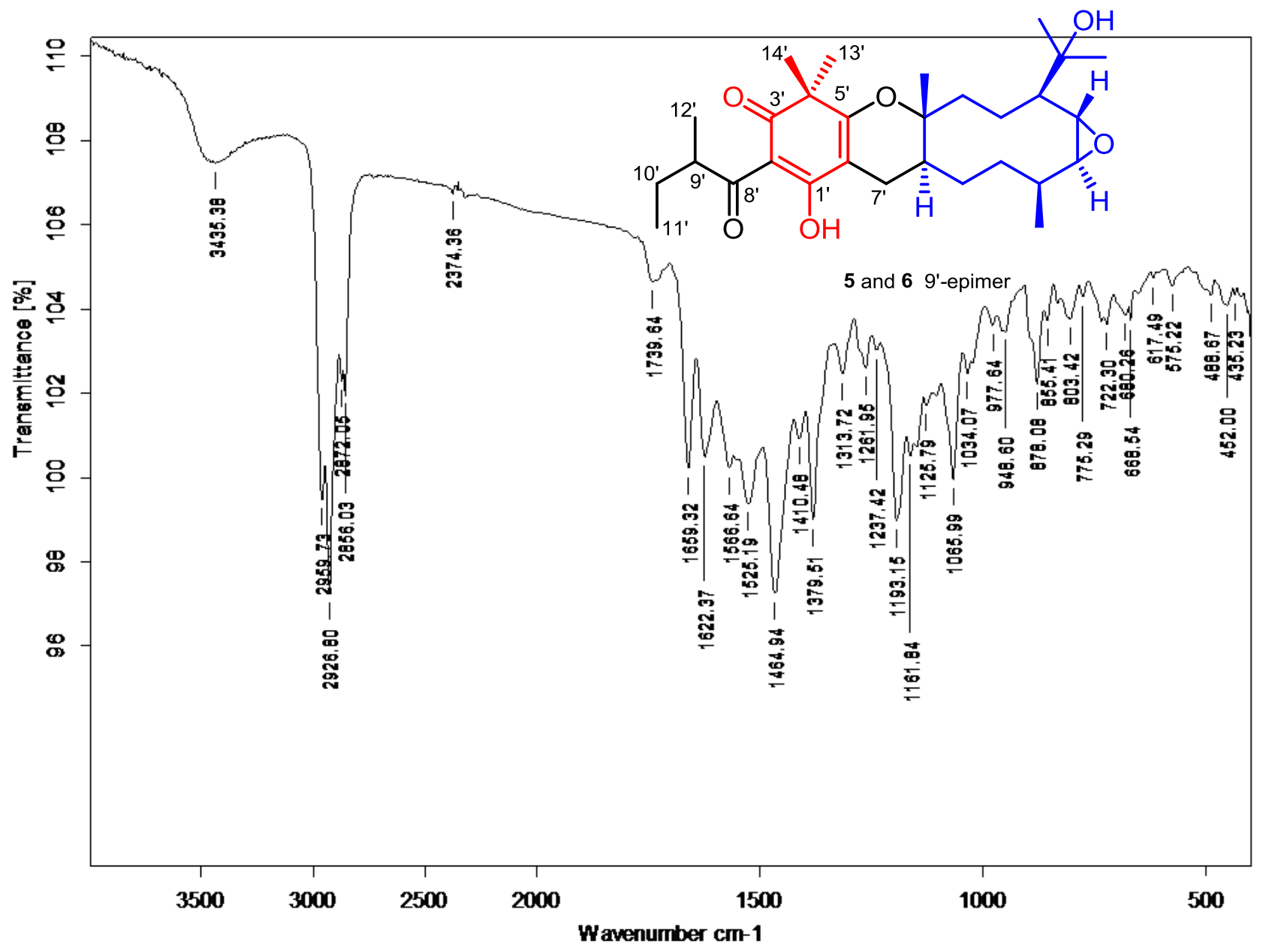


HRESIMS of compound 7

hlz2-18-2 \#23 RT: 0.33 AV: 1 NL: 3.68E6

T: FTMS + p ESI Full ms [50.00-1500.00]

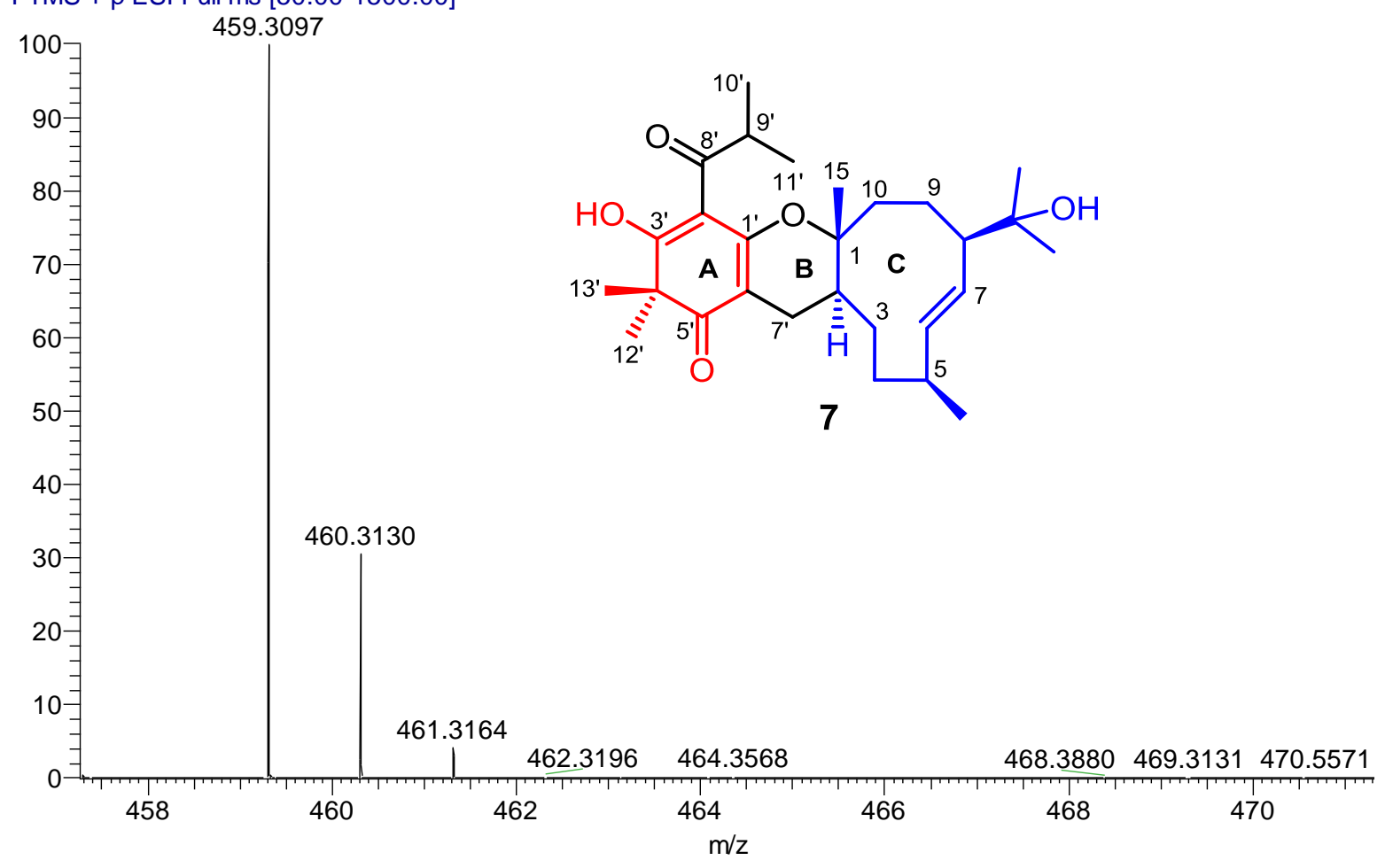


${ }^{1} \mathrm{H}$ NMR of compound 7 (in $\mathrm{CDCl}_{3}$ )

屯

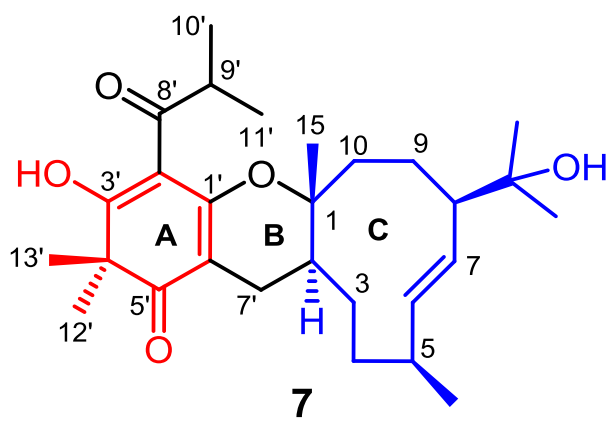

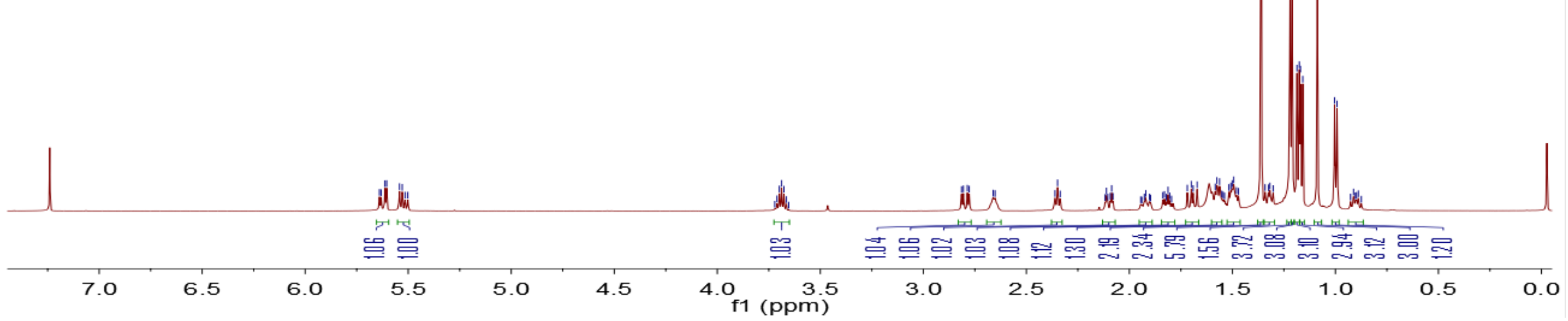


${ }^{13} \mathrm{C}$ NMR of compound 7 (in $\mathrm{CDCl}_{3}$ )
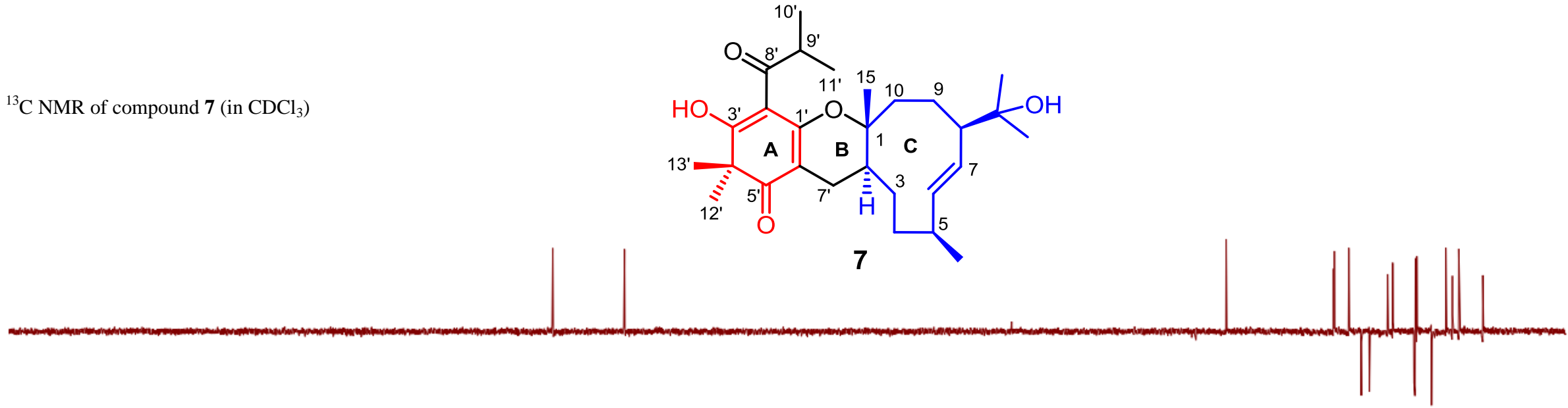

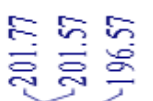

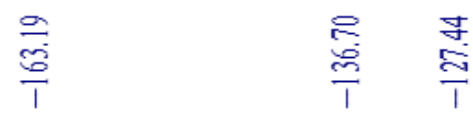

$\cong$
$\cong$

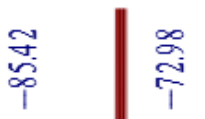

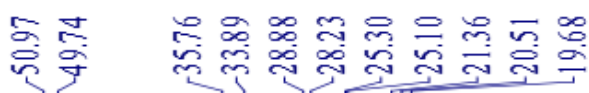

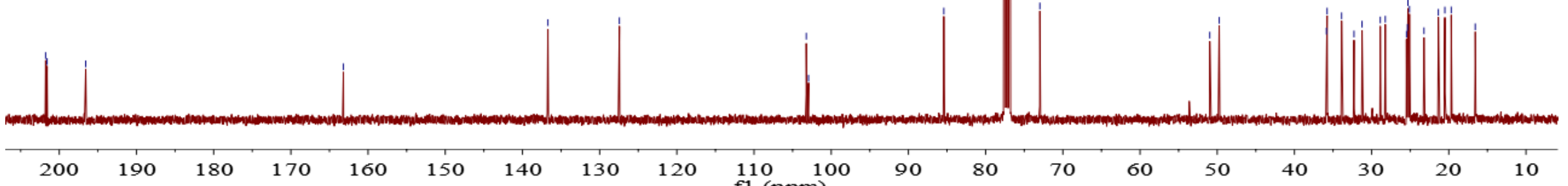


HSQC of compound 7 (in $\mathrm{CDCl}_{3}$ )

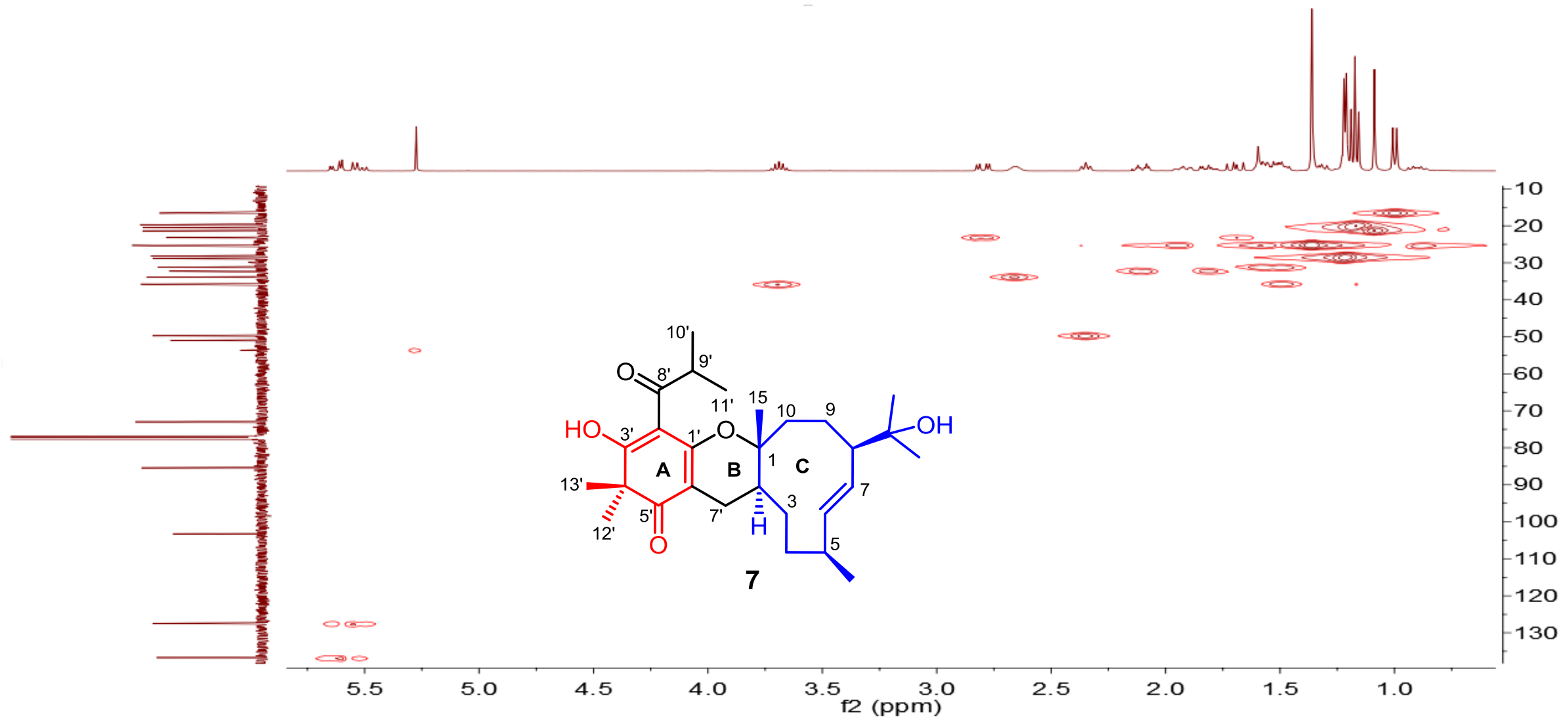


HMBC of compound 7 (in $\mathrm{CDCl}_{3}$ )

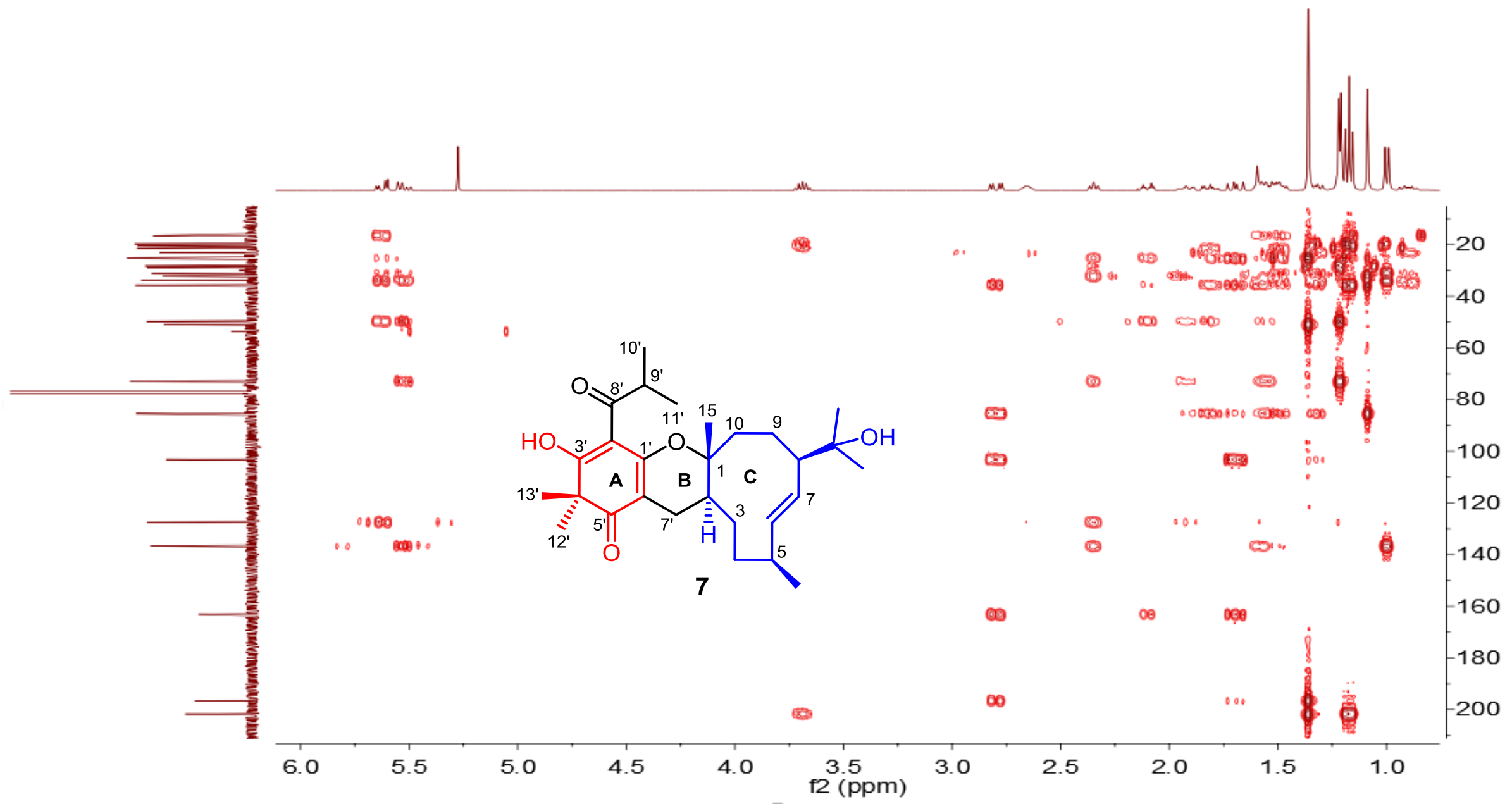




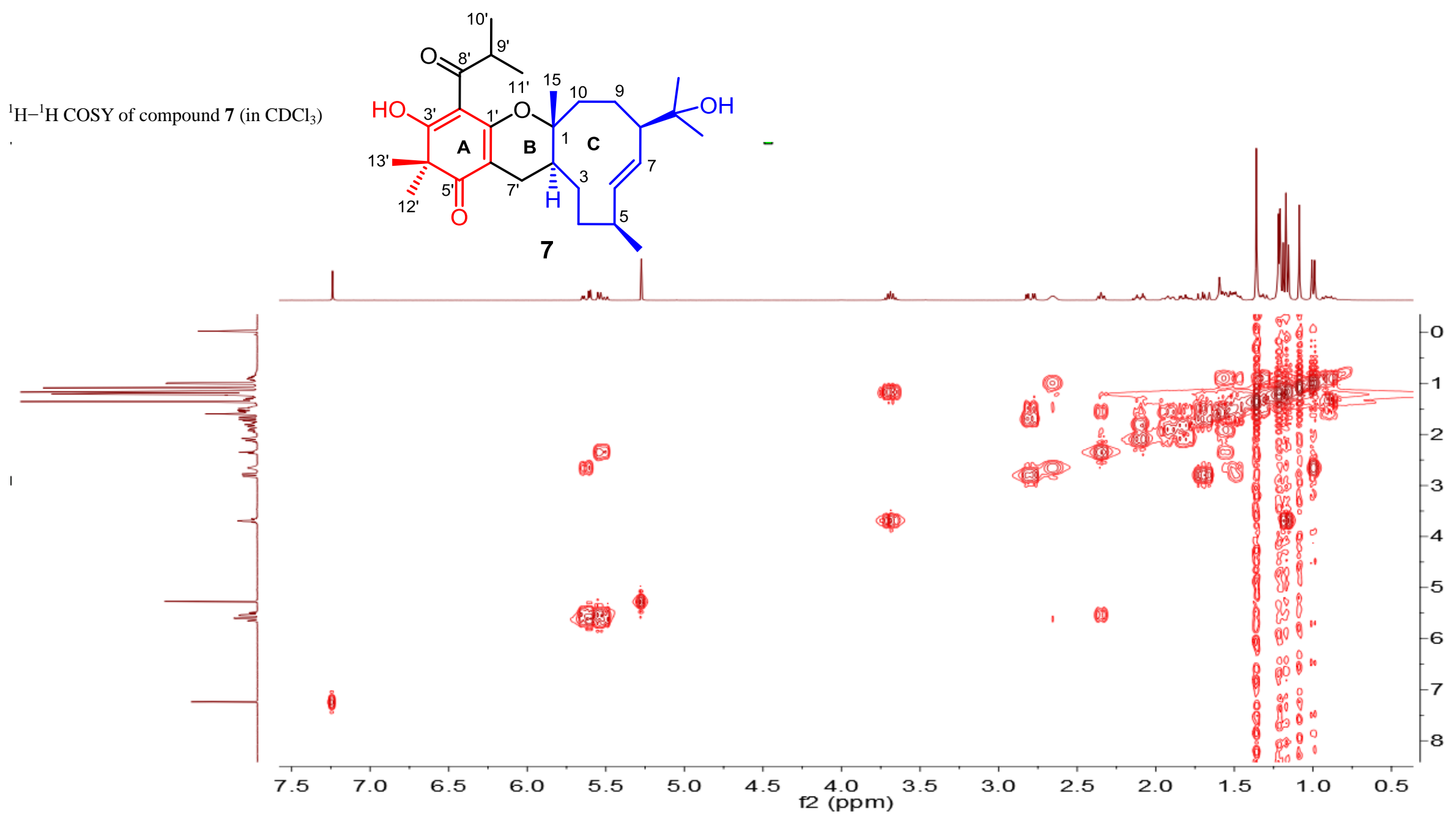




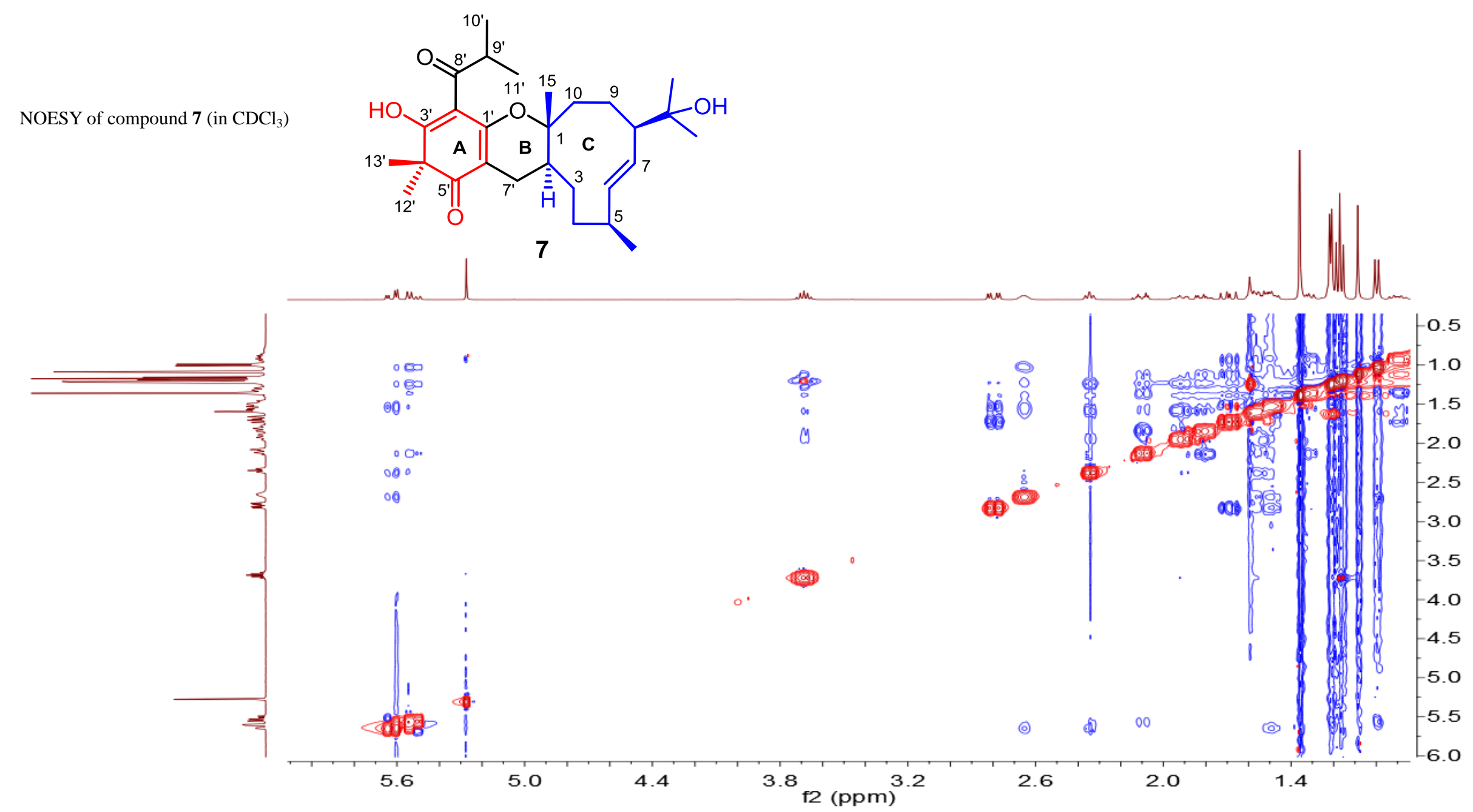


UV of compound 7 (in $\mathrm{MeOH}$ )

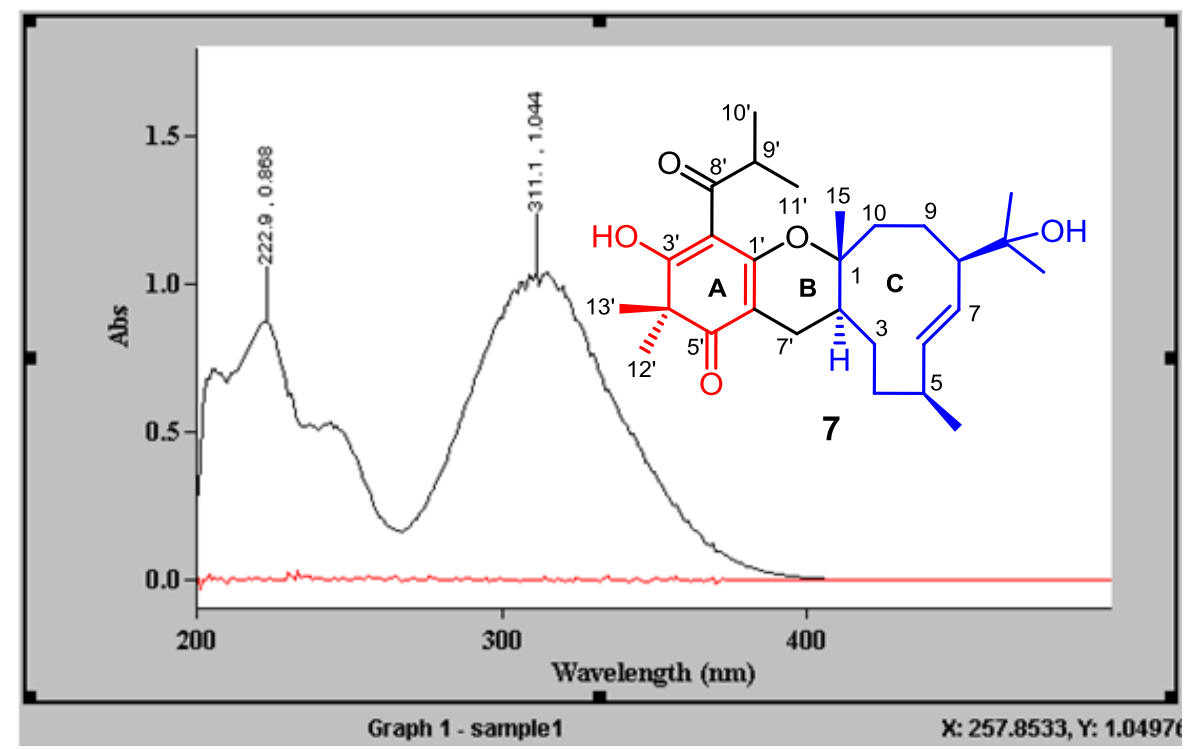




\section{IR of compound 7 ( $\mathrm{KBr}$ disc)}

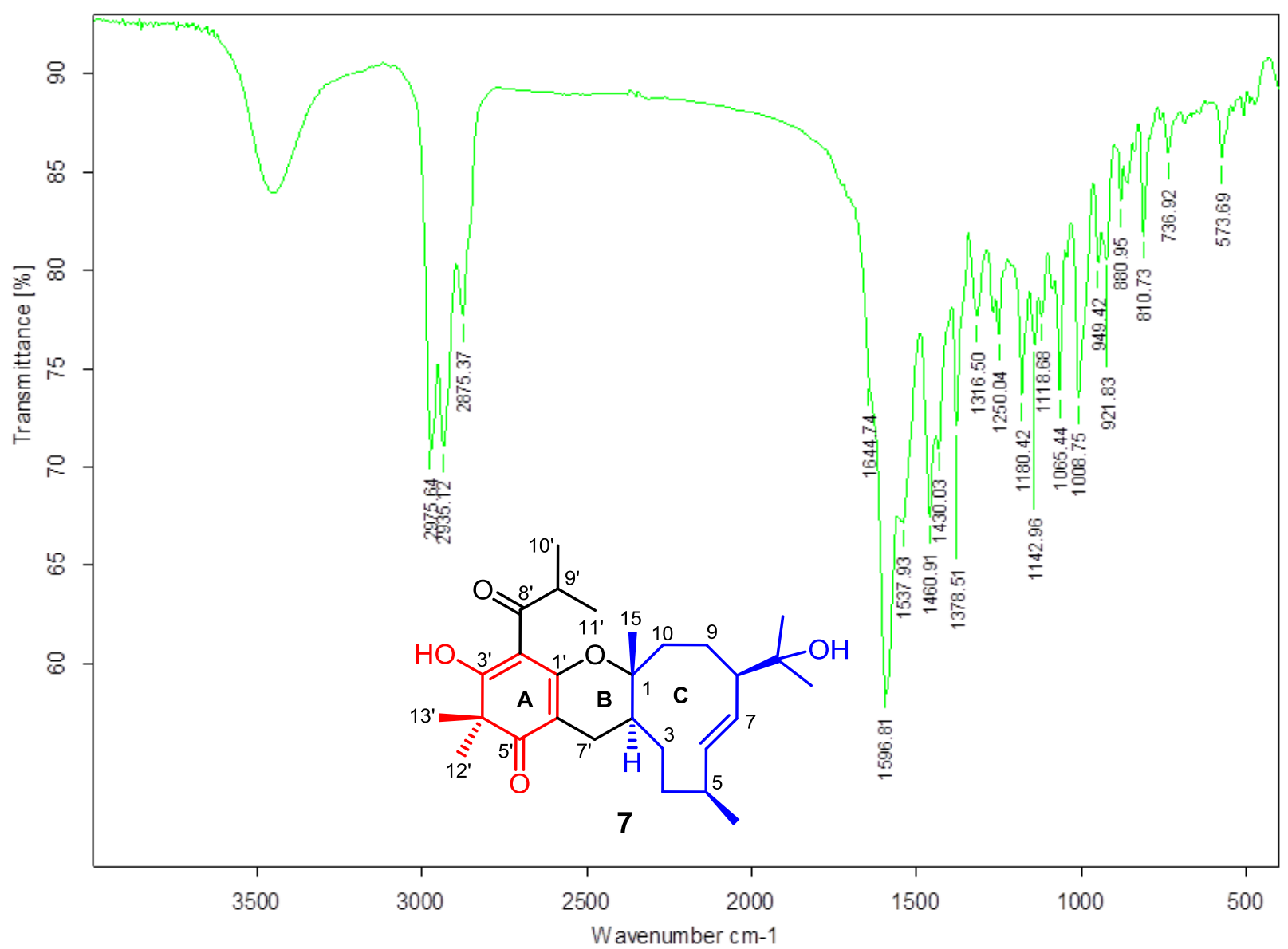

